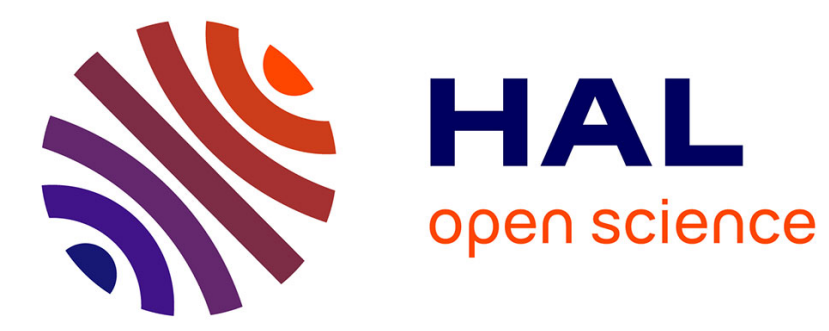

\title{
Recommandations pour le dimensionnement des évacuateurs de crues de barrages
}

\author{
P. Le Delliou, S. Aigouy, C. Albert, G. Degoutte, Luc Deroo, E. Domps, E. \\ Frossard, F. Fruchart, M. Lang, F. Lemperiere, et al.
}

\section{- To cite this version:}

P. Le Delliou, S. Aigouy, C. Albert, G. Degoutte, Luc Deroo, et al.. Recommandations pour le dimensionnement des évacuateurs de crues de barrages. CFBR, pp.166, 2013. hal-02598626

\section{HAL Id: hal-02598626 \\ https://hal.inrae.fr/hal-02598626}

Submitted on 16 May 2020

HAL is a multi-disciplinary open access archive for the deposit and dissemination of scientific research documents, whether they are published or not. The documents may come from teaching and research institutions in France or abroad, or from public or private research centers.
L'archive ouverte pluridisciplinaire HAL, est destinée au dépôt et à la diffusion de documents scientifiques de niveau recherche, publiés ou non, émanant des établissements d'enseignement et de recherche français ou étrangers, des laboratoires publics ou privés. 


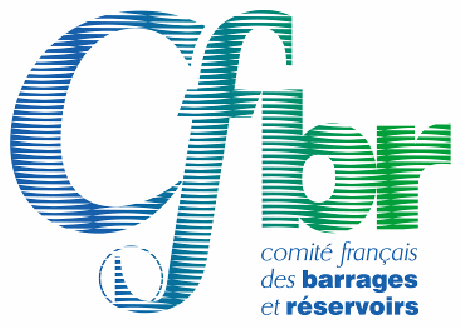

Groupe de Travail «Dimensionnement des évacuateurs de crues de barrages »

\section{Recommandations pour le dimensionnement des évacuateurs de crues de barrages}




\section{Objectifs du groupe de travail (lettre de mission du CFBR du 9 juillet 2009)}

L'objectif essentiel du groupe de travail est de proposer des principes de dimensionnement des évacuateurs de crues. Pour ce faire il devra:

- définir les concepts clés de la sécurité des barrages en période de crue, et préciser le vocabulaire afférent (cotes de PHE, de danger, revanche,...etc. et crues correspondantes); ces concepts tiendront compte de la classe et du type des barrages,

- proposer des recommandations pour la prédétermination des crues extrêmes, la prise en compte du laminage, l'impact de réservoirs amont,...

- proposer les critères de dimensionnement correspondant aux différentes sollicitations retenues; ce dimensionnement concerne l'ensemble des organes utilisés pour le transit des crues,

- proposer des principes généraux de dimensionnement et de diagnostic des organes vannés et de leurs systèmes de commande.

Remarques complémentaires :

- une synthèse de quelques pratiques étrangères, et en particulier celles qui ont évolué récemment, pourrait être un travail initial du GT, facilitée par les publications $d u$ récent colloque de 2009 ;

- le groupe prendra en compte les publications du Cfbr existantes : "Petits Barrages recommandations pour la conception, la réalisation et le suivi » Cfgb 1997, et "Recommandations pratiques pour améliorer la sécurité des barrages en crue » Cfgb 1998 ;

- le choix de la crue de projet pendant des périodes de travaux pour protéger les installations de chantier ne fait pas partie explicitement de la mission du groupe. Ce point pourra être traité néanmoins par le groupe s'il en ressent le besoin.

\section{Composition du GT}

Ce document a été rédigé par un groupe de travail du Comité Français des Barrages et Réservoirs (CFBR) qui a mené cette tâche sur la période de septembre 2009 à juin 2012 (15 réunions). Le groupe était composé de personnalités désignées en fonction de leurs compétences et expériences dans le domaine du dimensionnement des évacuateurs de crues :

Patrick Le Delliou,

Stéphan Aigouy,

Claude Albert,

Gérard Degoutte

Luc Deroo,

Eric Domps,

Etienne Frossard,

Francis Fruchart,

Michel Lang,

François Lemperière ${ }^{1}$,

Daniel Loudière,

Bernard Reverchon,

Paul Royet,

Thibaut Ulrich,

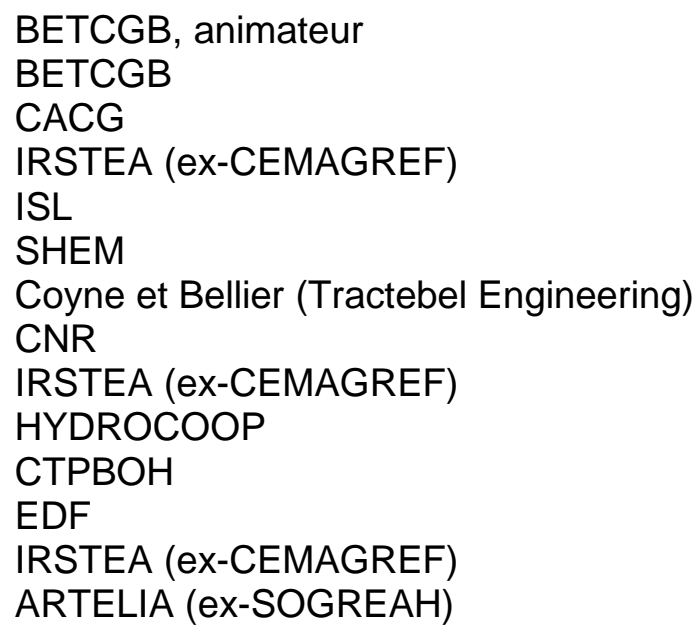

Stéphan Aigouy a assuré le secrétariat du groupe de travail.

\footnotetext{
${ }^{1} \mathrm{~N}$ 'approuve pas le rapport du groupe de travail
} 


\section{SOMMAIRE}

Introduction

Pourquoi des recommandations pour le dimensionnement des évacuateurs de crues de barrages?

Domaine d'application des recommandations

Démarche générale du dimensionnement des évacuateurs de crues de barrages

1. Terminologie - Glossaire

1.1. Géométrie

1.1.1 Cote de retenue normale $(\mathrm{RN})$

1.1.2 Cote de protection

1.1 .3 Cote des plus hautes eaux (PHE)

1.1.4 Cote de danger

1.1.5 Revanche

1.1.6 Tirant d'air

1.2. Hydrologie

1.2.1 Régime de la rivière

1.2.2 Variables caractérisant les crues

1.2.3 Probabilité de non-dépassement $p$ et période de retour $T \_12$

1.2.4 Crue maximale probable 13

1.2.5 Crues de référence pour les projets __ 13

1.3. Organes hydrauliques __ 14

1.3.1 Evacuateur de crues — 14

1.3.2 Organe de vidange 14

1.3.3 Organe de prise__ 14

1.3.4 Débitance__ 14

2. Recommandations pour l'évaluation des crues___ 15

2.1. Difficultés liées à l'estimation des crues extrêmes __ 15

2.2. Déroulement d'une étude des crues extrêmes __ 15

2.3. Collecte des données

2.3.1 Information hydrométrique 16

2.3.2 Information pluviométrique 16

2.3.3 Données naturalistes__ 18

2.3.4 Études hydrologiques antérieures __ 19

2.3.5 Mode de fonctionnement du bassin en crue ___ 19

2.4. Prédétermination des crues extrêmes sur un site jaugé __ 19

2.4.1 Méthodes basées sur les débits _ 20

2.4.2 Méthodes basées sur la modélisation des processus de transformation pluie-débit — 21

2.5. Méthodes de transfert vers un site non jaugé __ 23

2.5.1 Formules régionales 24

2.5.2 Méthode de l'indice de crue___ 25

2.5.3 Méthode Shyreg __ 25

2.6. Pluie Maximale Probable / Crue Maximale Probable $\quad 26$

2.6.1 Estimation de la PMP 26

2.6.2 Estimation de la crue maximale probable __ 28

2.7. Propositions pour la prédétermination des crues extrêmes__ 29

3. Recommandations pour les dispositions constructives et les modalités de maintenance et d'utilisation des évacuateurs de crues__ 31

3.1. Démarche globale pour garantir la fiabilité ___ 31 
3.2. Systèmes concernés ___ 33

3.2.1 Types d'évacuateur — 33

3.2.2 Dimensionnement des évacuateurs vis-à-vis des corps flottants ___ 42

3.2.3 Coincement des vannes par colmatage du pied des vannes __ 49

3.2.4 Types de vannes les plus répandus _ 50

3.2.5 Organes de manœuvre __ 57

3.2.6 Systèmes de contrôle-commande__ 59

3.2.7 Commandes hydrauliques à partir de la retenue __ 60

3.3. Conception des équipements_ 60

3.3.1 Équipements électromécaniques — 60

3.3.2 Commande des vannes__ 65

3.3.3 Acquisition des informations _ 69

3.3.4 Système de surveillance et de conduite ___ 71

3.4. Suivi et maintenance _ 75

3.4.1 Principes généraux 75

3.4.2 Essais périodiques des organes __ 75

3.4.3 Contrôles périodiques __ 76

3.4.4 Opérations de maintenance _ 77

3.4.5 Requalification des ouvrages et matériels___ 77

3.5. Utilisation des évacuateurs de crues - Gestion du passage des crues__ 77

3.5.1 Consigne générale d'évacuation des crues __ 79

3.5.2 Consigne d'exploitation en crue ___ 79

3.6. Retours d'expérience sur des cas de défaillance ___ 80

3.6.1 Défauts de conception___ 81

3.6.2 Défauts des informations entrantes du système de gestion ___ 81

3.6.3 Défaut de maintenance ___ 82

4. Recommandations pour le dimensionnement des évacuateurs de crues 84

4.1. Pratiques françaises actuelles pour le dimensionnement des évacuateurs neufs ou existants

4.1.1 Préambule 84

4.1.2 Cote des PHE et revanche 84

4.1.3 Crues de dimensionnement 84

4.1.4 Écrêtement par la retenue 85

4.1.5 Dimensionnement hydraulique de l'évacuateur de crues _ 85

4.1.6 Dimensionnement hydraulique des coursiers, organes de dissipation..._ 85

4.1.7 Analyse du risque «crues » pour les PPI ___ 86

4.1.8 Cas des petits barrages___ 86

4.2. Réglementations et pratiques étrangères _

4.2.1 Documentation__ 87

4.2.2 Classement des barrages ___ 88

4.2.3 Crues considérées___ 88

4.3. Propositions pour le dimensionnement ou la vérification des évacuateurs de crues en fonction de la classe (vulnérabilité), du type de barrage et du type d'évacuateur

4.3.1 Cotes de référence 91

4.3.2 Situations de projet 93

4.3.3 Dimensionnement hydrologique _ 96

4.3.4 Dimensionnement hydraulique 104

4.3.5 Dimensionnement structurel et mécanique ___ 109

4.3.6 Cas particulier des barrages mobiles en rivière ___ 111

4.4. Adaptation des barrages existants aux nouvelles recommandations _ 114

4.5. Utilisation des recommandations en dehors du contexte français _ 115

4.5.1 Période de retour de la crue extrême __ 115

4.5.2 Très petits barrages___ 116 
5. Axes de recherches et de développements 117

5.1. Notion de ligne d'eau de danger

5.2. Prise en compte des incertitudes pour le choix de l'aléa hydrologique de référence

5.3. État initial de remplissage du barrage et volume de la crue de dimensionnement ou de la crue extrême.

5.4. Estimation des crues extrêmes

5.5. Nouvelles problématiques posées par l'approche des états limites ultimes 126

5.6. Détermination de l'occurrence des crues de danger par la méthode des dommages incrémentaux

5.6.1 Introduction 128

5.6.2 Principe de la méthode telle qu'appliquée au Québec 129

5.6.3 Traduction graphique de détermination de la crue de danger __ 131

5.6.4 Conduite pratique de la méthode

5.6.5 Applicabilité de la méthode en France

Annexe 1 : Pratiques étrangères 136

Annexe 2 : Calcul de la revanche due aux vagues 149

Annexe 3 : Quelques exemples d'adaptation d'évacuateurs de crues de barrages existants 


\section{Introduction}

\section{Pourquoi des recommandations pour le dimensionnement des évacuateurs de crues de barrages?}

La maîtrise du passage des crues est un élément fondamental de la sécurité des barrages ; elle est en grande partie fonction du dimensionnement des évacuateurs de crues et de leur fonctionnement. Les pratiques françaises en la matière relèvent actuellement beaucoup de l'expérience des différents bureaux d'études et maîtres d'ouvrages.

Deux colloques organisés en commun avec la SHF, en 2006 et en 2009, ont constitué autant d'étapes pour mieux appréhender cette problématique "crues" et initier les échanges techniques sur le sujet.

Le Comité Français des Barrages et Réservoirs a souhaité concrétiser ces échanges et a décidé en conséquence la mise en place d'un groupe de travail chargé de proposer à la Commission Exécutive des recommandations pour le dimensionnement des évacuateurs de crues applicables au parc des barrages français. Ces recommandations devaient tenir compte du contexte de la nouvelle réglementation française et en particulier au besoin de cadre de cohérence attendu par les rédacteurs et vérificateurs des études de dangers. Enfin, les résultats de ces réflexions menées au sein du CFBR répondent également à une attente du Comité Technique Permanent des Barrages et des Ouvrages Hydrauliques (CTPBOH).

\section{Domaine d'application des recommandations}

Ces recommandations s'appliquent à la justification du dimensionnement des capacités d'évacuation des crues de tous les barrages de classes A à D (décret 2007-1735 du 11 décembre $2007^{2}$ ) dans le contexte français, quel que soit le type de barrage et le type d'organe d'évacuation. Pour les barrages de classe $\mathrm{D}$, il a toutefois été souhaité limiter le champ d'application des recommandations aux barrages retenant au moins $50000 \mathrm{~m}^{3}$; les barrages de classe $D$ dont la retenue est plus petite et a fortiori les barrages non classés ne sont pas concernés par les présentes recommandations. Le document est destiné aux projets d'évacuateurs de crues de barrages neufs, ainsi qu'aux projets de révision de barrages anciens et aux vérifications d'évacuateurs de barrages en service.

La majorité des recommandations du présent rapport, élaborées en fonction du parc de barrages et du contexte social et législatif français, pourrait être appliquée dans d'autres pays. Toutefois, les critères de dimensionnement, notamment ceux qui sont proposés au chapitre 4.3, doivent être considérés comme spécifiques et leur application à d'autres pays imposerait vraisemblablement des adaptations.

\section{Démarche générale du dimensionnement des évacuateurs de crues de barrages}

Dans ces recommandations, on examine successivement la terminologie relative au domaine et utilisée tout au long des recommandations, l'évaluation des crues, les

\footnotetext{
${ }^{2}$ Pour mémoire, les classes de barrages sont définies par ce décret en fonction de la hauteur du barrage et de la valeur du critère $\mathrm{H}^{2} \mathrm{~V}^{0,5}$, avec une valeur de 200 entre la classe $\mathrm{B}$ et la classe $\mathrm{C}$, et de 20 entre la classe $C$ et la classe D.
} 
dispositions constructives et les modalités de maintenance et d'utilisation des évacuateurs de crues, le dimensionnement des évacuateurs de crues et les axes de recherches.

Le logigramme à utiliser dans toute démarche de dimensionnement d'un évacuateur de crues est le suivant :

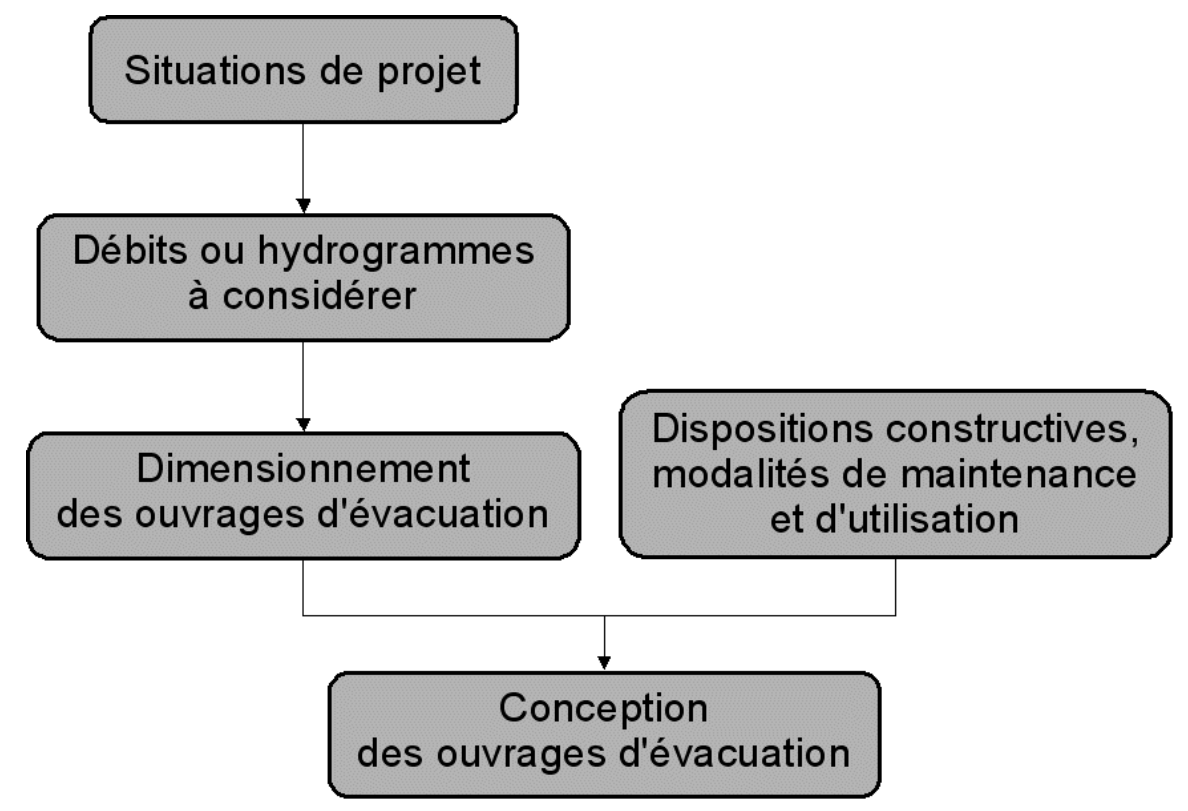

Démarche générale pour le dimensionnement et la conception d'un évacuateur de crues

Les différentes situations de projet à examiner sont définies au chapitre 4.3.2 (situation normale d'exploitation, situation rare de crue, situation exceptionnelle de crue, situation extrême de crue, autres situations de projet).

Les périodes de retour à considérer sont définies au chapitre 4.3.3. Les débits ou hydrogrammes de crue sont établis selon une méthodologie explicitée au chapitre 2.

Le dimensionnement des évacuateurs de crues fait l'objet du chapitre 4.3.

Les dispositions constructives et les modalités de maintenance et d'utilisation font l'objet du chapitre 3. 


\section{Terminologie - Glossaire}

\subsection{Géométrie}

\subsubsection{Cote de retenue normale (RN)}

II s'agit de la cote maximale que la retenue peut atteindre en exploitation normale hors épisode de crue. Même si pour beaucoup de barrages il s'agit d'une cote physiquement matérialisée par la cote d'un seuil, par le sommet d'une vanne de surface..., ce n'est pas toujours le cas, comme par exemple pour un barrage qui ne serait muni que d'un évacuateur de fond ou de demi-fond. II s'agit généralement d'une valeur "contractuelle » fixée par un arrêté d'autorisation ou par un décret de concession.

À cette cote correspond un chapitre de la note de calcul de stabilité générale du barrage avec des coefficients de sécurité suffisants; en langage "Eurocodes ", il s'agit d'une vérification aux états limites en situation quasi-permanente (recommandations CFBR 2012 pour la justification de la stabilité des barrages-poids et CFBR 2010 pour la justification de la stabilité des barrages et des digues en remblais).

Pour les barrages non destinés spécifiquement à l'écrêtement des crues, la RN permet aussi de calculer le volume de la retenue nécessaire au classement de l'ouvrage vis-à-vis du décret 2007-1735.

\subsubsection{Cote de protection}

Cette notion ne s'applique qu'aux ouvrages de type écrêteurs de crues. II serait dangereux d'étendre cette notion aux barrages conçus pour d'autres usages : un barrage de retenue d'eau n'a pas a priori de rôle de protection, d'autant que les vitesses de variation de débits ne peuvent être réduites comme cela peut être le cas pour un barrage écrêteur de crues muni de plusieurs niveaux de seuils.

Un barrage écrêteur de crues est en général équipé de pertuis (libres ou vannés), dont le dimensionnement est basé sur la capacité du lit en aval, en particulier dans une traversée urbaine. On évalue généralement le débit maximal non dommageable du cours d'eau en aval du barrage (par exemple débit de plein bord du lit mineur ou débit d'un ouvrage aval créant section de contrôle) et on dimensionne le ou les pertuis inférieurs pour délivrer au maximum ce débit. Lors des crues, il y a stockage temporaire dans la tranche d'eau de laminage et évacuation d'un débit plafonné par ces pertuis, jusqu'à atteindre la cote d'un déversoir de surface dont la fonction est d'assurer la sécurité du barrage lors des événements extrêmes.

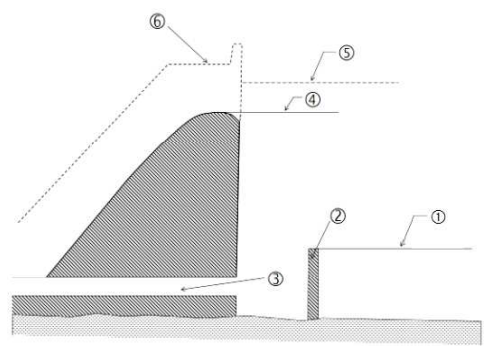
(1) Plan d'eau semi-permanent
(2) Batardeau
(3) Pertuis
(4) Seuil de surface (cote de protection)
(5) Cote des PHE
(6) Crête

Figure 1.1 - Coupe schématique d'un barrage écrêteur de crues 
La cote de protection est définie comme la cote du déversoir libre (sauf exception) de surface d'un barrage écrêteur de crues ; il s'agit d'une donnée intrinsèque à l'ouvrage.

La cote de protection est la cote de référence pour le calcul du volume « réglementaire » de la retenue au sens du décret 2007-1735. La note de calcul du barrage doit notamment faire apparaître une justification pour cette cote en situation rare.

\subsubsection{Cote des plus hautes eaux (PHE)}

La cote des PHE est introduite par la pratique française. Elle est parfois appelée « cote des plus hautes eaux exceptionnelles ».

Dans la pratique actuelle, elle correspond à la cote atteinte par la retenue dans l'hypothèse de l'arrivée d'une crue de dimensionnement de période de retour choisie.

À la cote des PHE, le calcul de stabilité de l'ouvrage doit faire apparaître des coefficients de sécurité suffisants (mais normalement inférieurs aux coefficients obtenus à RN). Par ailleurs, à cette cote, le passage des crues s'effectue sans endommagement de l'ouvrage et de l'ensemble des organes hydrauliques (évacuateurs de crues, coursiers, dissipateurs d'énergie, etc.).

Elle est liée à une crue, et donc à sa période de retour et à l'étude hydrologique qui en fournit les caractéristiques. On peut considérer qu'il s'agit d'une cote variable au cours de la vie de l'ouvrage.

\subsubsection{Cote de danger}

Elle s'est imposée au groupe de travail comme une composante obligatoire de l'estimation de la marge de sécurité d'un ouvrage.

Elle correspond à la cote au-delà de laquelle on ne sait plus garantir la stabilité de l'ouvrage. La vérification par le calcul de la stabilité intrinsèque du barrage pour cette cote est juste assurée avec les coefficients partiels associés aux situations extrêmes. Par ailleurs, à cette cote, le passage des crues s'effectue également sans causer la rupture de l'ouvrage (pas de ruine du barrage causée par le débordement d'un coursier, par l'érosion en pied due à la dissipation de l'énergie, etc.). Cela ne signifie pas qu'il y a rupture du barrage dès dépassement de cette cote. II y a en effet encore des marges "cachées " dans les méthodes de calcul (par exemple dans la notion de valeur caractéristique prise en compte dans les paramètres de résistance mécanique) et dans l'appréciation de l'ingénieur. En ce sens, la probabilité de rupture dès le dépassement de la cote de danger peut être significative mais sans être égale à 1 . La rupture dépend également encore de nombreux facteurs, comme par exemple l'ampleur et la durée du dépassement de la cote de danger (cas du déversement en crête d'un barrage meuble par exemple).

La détermination directe de cette cote pose d'importantes difficultés et il paraît inévitable d'adopter des règles forfaitaires (en tout cas pour fixer, par type de barrage, une borne supérieure) ; un état pathologique, un comportement particulier, un sous-dimensionnement de la structure peuvent justifier de retenir des valeurs inférieures. Selon les types 
d'ouvrages, la détermination de la cote de danger peut être entachée d'une incertitude plus ou moins forte ${ }^{3}$.

Par ailleurs, on peut parfois ne pas déterminer directement la valeur de la cote de danger, s'il peut être démontré que la stabilité du barrage reste assurée, avec les coefficients partiels associés aux situations extrêmes, pour la cote atteinte dans ces situations extrêmes. Cette cote est alors une borne inférieure pour la cote de danger.

Il est également possible que, dans certains cas particuliers, la cote de danger n'existe pas (par exemple pour un barrage dont la submersion fait rapidement monter la cote aval, conduisant à un fonctionnement proche d'un seuil en rivière).

\subsubsection{Revanche}

La revanche est la différence de cote entre la retenue et la crête de l'ouvrage. Elle peut, dans certains cas, inclure le parapet. Cette revanche est dimensionnée à partir de critères sur la hauteur des vagues (cf. 4.3.3.5 et annexe 2), tant par rapport à la $\mathrm{RN}$ que par rapport aux PHE. Elle n'est en aucun cas associée à la notion de sur-hauteur mise en place sur les barrages meubles ou sur fondation compressible pour faire face aux futurs tassements de l'ouvrage.

\subsubsection{Tirant d'air}

Le tirant d'air est la hauteur entre la surface libre d'un plan d'eau ou d'un écoulement et un obstacle situé au-dessus. Le tirant d'air est variable au cours du temps en fonction des débits, du niveau de la retenue... II peut dépendre aussi de la position des obstacles (vannes segments en position haute par exemple). Le tirant d'air permet d'apprécier le risque de coincement de corps flottants (la largeur des passes est tout aussi importante).

\subsection{Hydrologie}

\subsubsection{Régime de la rivière}

Les débits d'une rivière suivent un cycle saisonnier conditionné par le régime des précipitations et des températures, qui induisent des variations sur les écoulements, l'évapotranspiration, le stock neigeux et la couverture végétale, et par le niveau des nappes. On distingue classiquement la période des moyennes eaux de celle des extrêmes : basseseaux ou étiage, alimenté par une vidange de la nappe en l'absence de ruissellement de surface (par manque de fortes précipitations), et hautes-eaux ou crue.

Une crue naturelle est une intumescence du débit de la rivière provoquée par une précipitation et/ou une fonte de neige. L'eau qui coule dans une rivière en crue est en général chargée de sédiments boueux, sableux ou graveleux. Une crue accidentelle peut être provoquée par la rupture d'un ouvrage retenant un lac ou par la rupture d'une retenue temporaire provoquée par un embâcle ou un glissement de terrain.

\footnotetext{
3 Pour les barrages en remblai, pour lesquels l'effet seuil est plus marqué, la cote de danger sera vraisemblablement estimée avec une incertitude plus faible que pour les barrages-poids et $a$ fortiori pour les barrages-voûtes.
} 


\subsubsection{Variables caractérisant les crues}

Pour que l'ingénieur puisse en faire l'étude, on résume les crues par un certain nombre de grandeurs ou variables. Les variables principales sont les variables indispensables pour la sécurité des barrages car elles affectent principalement le niveau maximum de la retenue. Les variables secondaires ont en général un effet beaucoup moins important sur le niveau maximum de retenue, mais elles peuvent permettre de caractériser des facteurs aggravants liés à un fonctionnement spécifique en crue.

\subsubsection{Variables principales de caractérisation des crues}

Les variables principales de caractérisation des crues sont les suivantes:

- le débit de pointe $Q \max \left(\right.$ en $\mathrm{m}^{3} / \mathrm{s}$ ) : valeur maximale du débit entrant dans une retenue pendant la durée de la crue ;

- le volume de la crue (en $\mathrm{hm}^{3}$ ou millions de $\mathrm{m}^{3}$ ) : volume d'eau entrant dans une retenue pendant un épisode de crue. Le début et la fin de l'épisode n'étant pas toujours aisés à positionner (succession rapprochée d'épisodes de crue), on pourra donner par ailleurs le débit moyen maximal de la crue $V d$, pour différentes durées $d$;

- le temps de base de la crue (en $h$ ) : durée de la crue ou durée pendant laquelle le débit entrant dans la retenue est supérieur à une valeur donnée, en général la valeur du " débit de base » (qui peut être différent en début et en fin de crue). On peut aussi définir le temps de base comme, par exemple, la base du triangle de même débit de pointe et de même volume que la crue réelle.

II n'y a pas ambiguïté sur la définition du débit de pointe, mais le volume et le temps de base peuvent être estimés différemment par deux hydrologues suivant la méthodologie d'étude qui leur est propre, surtout en cas de crue complexe.

\subsubsection{Variables secondaires de caractérisation des crues}

On trouve également les variables secondaires suivantes :

- le débit de base avant et après la crue (en $\left.\mathrm{m}^{3} / \mathrm{s}\right)$ : avant la crue, on retient en général le débit de la rivière avant la brutale cassure qui marque souvent le début d'une crue. Après la crue, on retient souvent le débit qui apparaît sur une cassure de la courbe de décrue dessinée sur un graphique semi-logarithmique. En cas de crue complexe, il convient, pour estimer le débit de base, de soustraire le débit de fin de la crue précédente. Divers modèles permettent des estimations sinon plus fiables du moins plus homogènes d'une crue à l'autre. Une analyse des divers débits de base observés permet éventuellement de calculer un débit de base de la crue de projet. On retient parfois par précaution la moyenne annuelle ou saisonnalisée du débit du cours d'eau. La moyenne annuelle est également appelée module du cours d'eau. Une valeur plus forte pourra être retenue si l'on souhaite étudier le risque de concomitance d'un débit de base élevé avec un épisode de crue ;

- le débit moyen maximal $V d$ et le débit seuil maximal $Q d\left(e n \mathrm{~m}^{3} / \mathrm{s}\right)$ : pour une durée $d$ donnée, le débit moyen maximal $V d$ est obtenu en divisant le volume maximal de l'hydrogramme par la durée $d$ (recherche par moyenne glissante mobile). Le débit seuil maximal $Q d$ est le seuil de débit dépassé de façon continue sur la durée $d$. Le débit $V d$ est une variable intermédiaire utilisée dans la méthode du Gradex et ses variantes pour extrapoler la distribution des débits à l'aide de la distribution des pluies moyennes maximales $P d$, sur la même durée. Le débit $Q d$ peut être utilisé pour construire un 
hydrogramme de crue de référence, qui présente la particularité d'être homogène en fréquence, quel que soit le débit Vd utilisé pour le caractériser (cf. 5.3) ;

- les pluies journalières $(\mathrm{mm})$ : ce sont les pluies mesurées au pluviomètre, par relevé quotidien à heure fixe (7 ou 8 heures en général) ;

- les pluies moyennes maximales sur une durée $d$ : pour une durée $d$ donnée, il s'agit du cumul pluviométrique $(\mathrm{mm})$ ou de l'intensité $(\mathrm{mm} / \mathrm{h})$, mesurés au pluviographe ;

- l'hydrogramme de crue : pour un épisode de crue, variation du débit en fonction du temps. Pendant la phase de montée, le débit passe du débit de base au débit de pointe de crue. Puis il redescend jusqu'à la fin de la crue. On distingue l'hydrogramme d'entrée arrivant dans la retenue et l'hydrogramme de sortie restitué par les ouvrages d'évacuation du barrage. La forme de l'hydrogramme de crue pourra impacter le remplissage plus ou moins rapide de la retenue. On pourra s'intéresser à plusieurs paramètres pour caractériser la forme de l'hydrogramme : 1/ le rapport entre la pointe de crue Qmax et le débit moyen maximal Vd (appelé aussi coefficient de pointe) ; 2 / le gradient maximal de montée ou vitesse de montée de la crue $\left(\mathrm{m}^{3} / \mathrm{s} / \mathrm{h}\right)$ sur une durée $d$ (par moyenne glissante sur la montée de crue); $3 /$ les coefficients de forme et d'asymétrie qui caractérisent respectivement la concentration du volume autour de la pointe, et l'asymétrie du volume autour de la pointe.
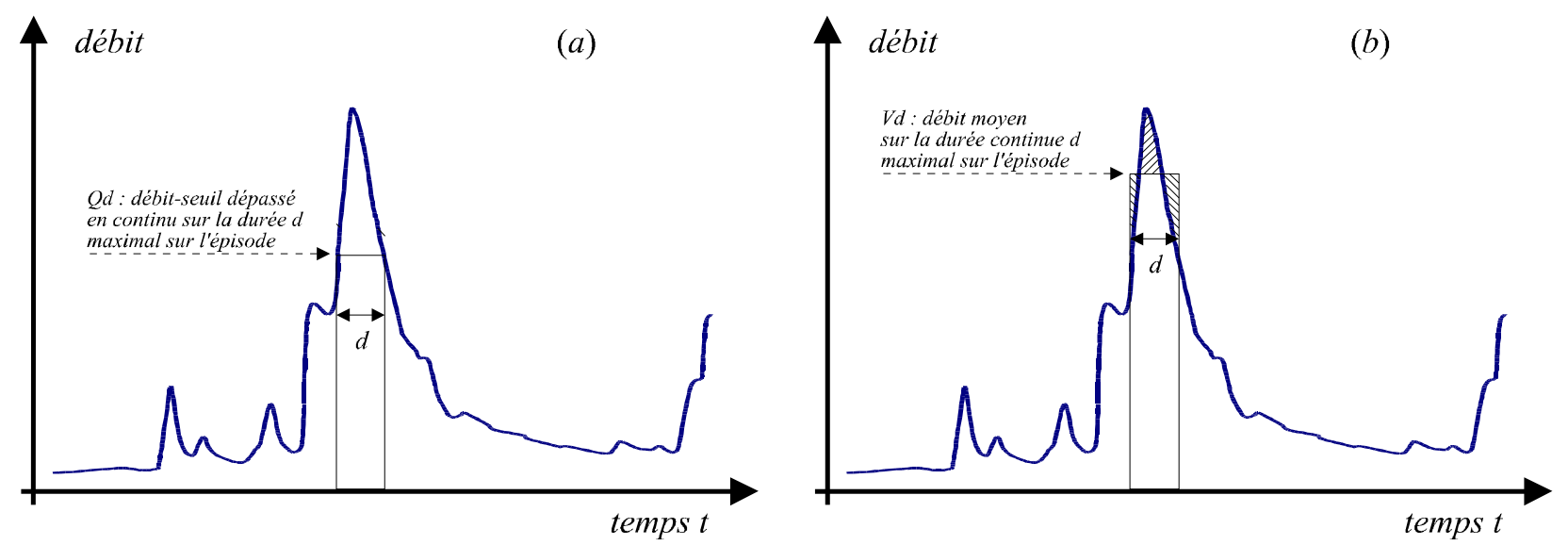

Figure 1.2 - Débit seuil et débit moyen maximal d'un hydrogramme de crue

\subsubsection{Probabilité de non-dépassement $p$ et période de retour $T$}

Si l'événement de référence est défini comme le dépassement d'un débit de référence $Q p$ par le maximum annuel, on peut lui associer deux grandeurs:

- la probabilité annuelle $p$ de non-dépassement du débit $Q p: p=\operatorname{Prob}[Q<Q p]$;

- le nombre moyen d'années $T$ (ou période de retour) séparant deux occurrences d'événement,

avec la relation $: p=1-1 / T$.

Le tableau 1.1 donne le risque d'observer au moins une fois un événement supérieur à la crue de référence sur une période donnée, à partir de la relation :

$$
\operatorname{Prob}[Q>Q p \text { au moins une fois sur } N \text { années }]=1-(1-1 / T)^{N}
$$


Après avoir défini le débit de référence utilisé pour caractériser la crue (débit de pointe, débit moyen maximal, débit seuil maximal), la crue décennale (respectivement centennale, millennale) est la crue dont la probabilité annuelle de dépassement du débit de référence vaut $10^{-1}$ (respectivement $10^{-2}, 10^{-3}$ ).

\begin{tabular}{|c|c|c|c|c|c|c|}
\hline \multicolumn{2}{|c|}{ Quantile Qp } & \multicolumn{5}{c|}{ Probabilité de dépasser au moins une fois } \\
le quantile Qp sur $N$ années
\end{tabular}

Tableau 1.1 - Probabilités de dépasser le quantile Qp

Les probabilités de dépassement à l'échelle annuelle peuvent sembler faibles, mais le risque est à considérer sur la durée de vie de l'ouvrage. Ainsi, la probabilité d'avoir au moins une crue millennale (respectivement décamillennale) sur une durée de 100 ans est d'une chance sur 10 (respectivement 100), ce qui est loin d'être négligeable.

La notion de période de retour est souvent interprétée à tort comme une récurrence régulière toutes les $T$ années d'un événement. II s'agit d'une durée moyenne entre deux occurrences. On peut citer le cas de la Loire en aval du bec d'Allier où les trois plus fortes crues connues depuis au moins deux siècles se sont produites sur une période de 21 ans, en 1846, 1856 et 1866.

On doit dans certains cas définir la crue de référence par son volume $V$, ou encore par son débit moyen maximal.

\subsubsection{Crue maximale probable}

En matière de sécurité des barrages, certaines pratiques font appel à la notion de Crue Maximale Probable ou possible (CMP). II s'agit d'une crue extraordinaire, envisageable mais non reliée à une probabilité.

\subsubsection{Crues de référence pour les projets}

On distingue plusieurs crues de référence :

- la crue de chantier : crue contre laquelle il est décidé de protéger le chantier durant la construction de l'ouvrage ;

- la crue de protection: pour les barrages écrêteurs de crues, la crue qui permet d'atteindre la cote de protection (cf. 1.1.2) ;

- la crue exceptionnelle : crue considérée lors de la détermination des PHE de l'ouvrage. Les PHE étant parfois susceptibles de varier au cours de la vie de l'ouvrage (nouvelle note de calcul de stabilité, modification de la capacité d'évacuation des crues...), la crue de dimensionnement est également variable : réactualisation de la crue exceptionnelle suite à un événement hydrologique extrême, modification de la probabilité à prendre en compte suite à une modification de la réglementation ; 
- la crue extrême : crue permettant à la retenue d'atteindre la cote de danger. Elle peut dépendre d'un taux de dysfonctionnement de l'évacuateur de crues. Attention, le terme " crue extrême » peut également être utilisé de manière générique, notamment par les hydrologues, pour caractériser les crues importantes, supérieures aux crues courantes (cf. 2.4 ou 2.7 par exemple).

\subsection{Organes hydrauliques}

\subsection{1 Évacuateur de crues}

II s'agit d'un organe hydraulique destiné à assurer le transit des crues. II peut s'agir d'un déversoir à seuil libre fonctionnant par «trop-plein » ou d'un organe vanné (de surface, de fond ou de demi-fond) ou d'un organe mixte ${ }^{4}$. Un même barrage peut avoir plusieurs organes hydrauliques dédiés à l'évacuation des crues, du même type ou non.

\subsubsection{Organe de vidange}

Il s'agit d'un organe hydraulique destiné à la vidange volontaire de la retenue (en cas de désordres graves, pour des opérations d'inspection, de maintenance...). L'organe de vidange est situé en partie basse du barrage. II peut ne pas être situé au point le plus bas du barrage (dans ce cas, la retenue ne peut pas être totalement vidée gravitairement et il reste un culot non vidangeable). L'organe de vidange n'est normalement pas compté comme évacuateur de crues (sauf s'il est explicitement conçu pour cela, avec des sécurités suffisantes pour la commande, l'alimentation électrique, etc.).

\subsubsection{Organe de prise}

II s'agit d'un organe hydraulique destiné à l'exploitation de l'eau de la retenue (prise usinière, prise d'eau potable...). II ne doit pas être considéré comme faisant partie des capacités d'évacuation des crues.

\subsubsection{Débitance}

La débitance d'un organe hydraulique est le débit évacuable pour une cote de retenue donnée. L'accroissement de cette débitance avec la cote de retenue peut être très variable en fonction du type d'évacuateur de crues (cf. 3.2.1). Le cas échéant, elle peut être influencée par les conditions d'ennoiement aval.

\footnotetext{
${ }^{4}$ Par exemple seuil libre équipé de hausses fusibles
} 


\section{Recommandations pour l'évaluation des crues}

\subsection{Difficultés liées à l'estimation des crues extrêmes}

L'estimation de la crue de dimensionnement et de la crue extrême d'un barrage pose des problèmes spécifiques liés au petit nombre et au type des données disponibles qui sont fournies par la nature suivant un processus aléatoire. De plus, les approches proposées pour analyser ces données sont diverses et font encore l'objet de recherches et parfois de débats. Très souvent, les séries hydrométriques à traiter ne couvrent que quelques dizaines d'années, avec en général aucun événement exceptionnel, et elles se trouvent à distance du barrage. On se trouve alors confronté à plusieurs difficultés :

- comment extrapoler des séries trop courtes, ne disposant le plus souvent d'informations que sur des événements plus ou moins courants?

- comment estimer des valeurs de référence sur l'ensemble du territoire alors que le réseau de mesure a une densité forcément limitée au regard de la variabilité spatiale des pluies et des débits ?

- comment mettre à jour notre connaissance des événements extrêmes dans un contexte évolutif, avec de fortes interrogations sur la variabilité climatique et les effets du changement climatique?

La théorie des valeurs extrêmes (Coles, 2001) offre un cadre mathématique rigoureux pour représenter le comportement probabiliste de la valeur maximale annuelle par la loi généralisée des extrêmes et des valeurs supérieures à un seuil par la loi de Pareto généralisée. Mais elle repose sur des hypothèses non complètement vérifiées en hydrologie (indépendance, homogénéité, stationnarité des valeurs de l'échantillon) et n'est valide que de façon asymptotique, pour des tailles d'échantillon de plusieurs centaines de valeurs bien supérieures à celles disponibles en pratique.

On est ainsi confronté à un fort niveau d'incertitude lié à la distribution d'échantillonnage (forte sensibilité à la présence de valeurs singulières), à l'erreur d'estimation sur les débits de crue (extrapolation de la courbe de tarage), et à l'extrapolation du modèle théorique aux valeurs extrêmes. Or les événements courants ne sont que peu informatifs, au sens statistique, pour la prédétermination des fortes valeurs de crues. La genèse physique de ces valeurs extrêmes est en effet bien différente de celle des événements courants. Appliquées sur de longues séries, la théorie des valeurs extrêmes redevient pertinente pour l'estimation des valeurs extrêmes, d'où la préoccupation des hydrologues de développer des méthodes permettant d'incorporer des informations complémentaires aux seules séries locales de données de quelques dizaines d'années (cf. 2.4).

\subsection{Déroulement d'une étude des crues extrêmes}

Après une phase de recherche des données disponibles, on distingue le cas où les données hydrométriques sont disponibles près du site à étudier de celui où les données sont insuffisantes ou inexistantes. Dans le premier cas de figure, il sera possible de réaliser une estimation de crues de référence, en exploitant des informations sur les débits et des outils basés sur la connaissance des processus de transformation de la pluie en débit. Une analyse régionale est souhaitable même dans le cas où les données sont disponibles sur le site car elle renforce la fiabilité de l'étude. 
Dans le second cas de figure, il est nécessaire d'utiliser des approches de transfert spatial pour estimer l'aléa hydrologique de référence sur un site non jaugé, ou avec une information réduite.

\subsection{Collecte des données}

\subsubsection{Information hydrométrique}

\subsubsection{Constitution d'un jeu de données}

Afin de constituer un jeu de données, il convient de :

- récupérer la chronique de débit sur le site le plus proche, et sur des sites voisins ;

- vérifier l'existence de données sur les crues anciennes auprès du service de prévision des crues, de la Dreal (atlas des zones inondables) et en consultant les études hydrologiques antérieures et l'inventaire de Maurice Champion (Champion, 2002) ;

- se renseigner sur l'existence de marques de crues ;

- compléter éventuellement ces informations par une enquête historique spécifique (consultation des archives départementales et parcours de fonds plus spécialisés).

\subsubsection{Analyse critique des données}

L'analyse critique des données contient les étapes suivantes:

- visualisation de la chronique de débit ;

- examen des courbes de tarage et du nuage des jaugeages ;

- échange avec le gestionnaire sur la qualité des données ;

- vérification de la cohérence des informations (recoupement spatial, analyse des laisses de crue) ;

- dans la mesure du possible, examen du dossier de la station, visite de terrain et validation de l'extrapolation de la courbe de tarage ;

- conversion des cotes de crues historiques en débit (vérifier la stabilité du fond du lit et prendre en compte d'éventuels changements dans la géométrie du lit mineur et/ou du lit majeur) ;

- détermination d'un seuil d'exhaustivité au-delà duquel on peut considérer que tous les événements sont connus.

\subsubsection{Information pluviométrique}

\subsubsection{Constitution d'un jeu de données pluviométriques}

À défaut de pouvoir collecter toutes les observations journalières aux stations de la zone d'étude, on recueille des totaux et maxima mensuels et annuels de pluies journalières aux pluviomètres de la zone d'étude, pluies journalières supérieures à un seuil pendant la période d'observation des crues (aux pluviomètres utiles pour l'étude pluie-débit). On ajoute éventuellement les enregistrements pluviographiques des fortes averses.

L'application d'approches par maximisation de la pluie (cf. 2.6.1.1) peut exploiter des informations complémentaires aux précipitations comme l'humidité relative, la température de l'air et la vitesse du vent. La méthode Schadex (cf. 2.4.2.3) exploite des informations sur 
les champs de pression (approche par type de temps) et sur la température de l'air (correction de la pluie de bassin sur les zones d'altitude, et modèle hydrologique Mordor avec une composante nivale).

\subsubsection{Analyse critique des données}

On procède en trois étapes:

- Étape 1: vérification de lacunes d'observations, par comparaison des données de stations proches; en cas de lacunes constatées, soit on supprime l'année, soit on reconstitue les valeurs manquantes (à condition que la somme des valeurs reconstituées soit inférieure à 20 ou $30 \%$ du total annuel). Les fortes pluies journalières ne sont jamais reconstituées ;

- Étape 2: constitution de séries longues et aussi complètes que possible à partir des observations en des lieux différents. Cette étape est souvent réalisée par l'organisme gestionnaire du réseau, qui met bout à bout sous le nom d'une station unique les séries observées en des postes différents ;

- Étape 3: contrôle de l'homogénéité des séries obtenues, par doubles cumuls par exemple. En cas de rupture d'homogénéité, soit on coupe la série en deux, soit on corrige une des deux parties de la série. Ce contrôle s'effectue sur chaque série de totaux annuels de pluie (qui sont donc à collecter même pour une étude des crues) et, en cas d'hétérogénéité constatée, le résultat est vérifié ou infirmé sur la série des maxima annuels de pluies journalières.

Un contrôle des enregistrements pluviographiques est également indispensable. Dans certains cas, un plafonnement des intensités de pluie à pas de temps court peut être observé du fait de problèmes métrologiques (cadence de basculement des augets insuffisante, surface du cône récepteur trop importante, déficit de captation liée au vent, etc.).

\subsubsection{Analyse de la pluie du bassin considéré}

Il convient :

- d'analyser la variabilité spatiale des pluies dans le secteur géographique d'étude (au-delà des limites du bassin versant) et éventuellement prendre en compte des phénomènes particuliers : effet orographique, abattement des précipitations en zone d'altitude (limite pluie-neige) ;

- de raisonner suivant les approches sur une lame d'eau de bassin (obtenue par moyenne arithmétique de plusieurs postes ou par krigeage), ou de retenir un poste représentatif de la pluie de bassin.

\subsubsection{Choix d'un pas de temps et d'un échantillonnage saisonnier adaptés à la dynamique des crues du bassin considéré}

II convient :

- du fait de la meilleure disponibilité des pluies journalières (plus forte densité du réseau d'observation, longueur des chroniques), de retenir un pas de temps supérieur ou égal à la journée, en effectuant si besoin une correction sur le centrage des valeurs (correction de Weiss). Si le temps de réaction du bassin en crue est de quelques heures, il est indispensable d'exploiter directement des données pluviographiques ou d'utiliser des courbes intensité-durée-fréquence ; 
- de raisonner sur la saison à plus fort risque plutôt qu'à l'échelle annuelle. Dans le cas de méthodes d'extrapolation basées sur l'information pluviométrique, ne pas retenir la saison des plus fortes intensités de pluie si celle-ci ne correspond jamais à la saison des plus fortes crues (cas de pluies orageuses d'été provoquant des débits inférieurs à ceux des crues hivernales).

Nota: certaines méthodes, comme la méthode Speed (cf. 2.4.2.2), intègrent la relation intensité-durée-fréquence des pluies dans la relation probabiliste pluie-débit, ce qui justifie dans ce cas l'utilisation des seules pluies journalières.

\subsubsection{Critique des données et des hypothèses de modélisation pluie-débit}

La critique suit la démarche suivante :

- contrôle visuel de la chronique des pluies et de celle des crues et comparaison des données agrégées à différents pas de temps (journalier $\rightarrow$ mensuel; horaire $\rightarrow$ journalier) ;

- raisonnement en sous-bassins homogènes d'un point de vue climatologique, si l'échelle du bassin est trop large ;

- étude du rendement des pluies (lame d'eau ruisselée / lame d'eau précipitée) des plus forts événements de crue, pour évaluer le seuil d'extrapolation des méthodes statistiques (Gradex, Agregee, Speed) ;

- vérification si l'hypothèse de correspondance statistique entre les distributions de pluie et de débit d'un même pas de temps est pertinente, et le cas échéant utilisation d'une modélisation plus complète (influence de la neige, présence de karst, crue par remontée de nappe, laminage des crues par une zone d'expansion ou par des ouvrages de retenue, secteur urbanisé...) ;

- vérification de la qualité du calage du modèle pluie-débit des méthodes par simulation (Shypre), sur les plus forts événements de crue.

\subsubsection{Données naturalistes}

L'approche hydrogéomorphologique (Garry et al., 1996) permet, à partir d'une analyse des cartes topographiques au $1 / 25000^{\mathrm{ème}}$, de stéréographies aériennes et de reconnaissances de terrain par un géomorphologue, de distinguer les différentes unités du lit d'écoulement de la rivière : le lit mineur correspond à la section usuelle d'écoulement, le lit moyen assure la transition entre le lit mineur et le lit majeur, et le lit majeur, qui fonctionne pour les crues importantes, correspond à la plaine alluviale. Les limites de ce dernier lit donnent une idée de l'extension maximale du champ d'inondation. Cette méthode a fait l'objet d'une application généralisée à l'ensemble de la région Midi-Pyrénées (Prunet, 1998 ; Vidal, 2000 ; Lambert et al., 1995) et des principaux cours d'eau des régions Languedoc-Roussillon et Provence-Alpes-Côte-d'Azur (Delgado, 2006), dans le cadre de la mise en place d'une cartographie informative sur les zones inondables.

Les crues récentes de novembre 1999 dans l'Aude et de septembre 2002 dans le Gard ont confirmé pour les cours d'eau méditerranéens que les inondations pouvaient réoccuper l'ensemble de leurs plaines alluviales. Elles ont également permis de vérifier la pertinence de la cartographie des plaines alluviales et des zones inondables par approche hydrogéomorphologique. Une estimation des débits des crues de référence hydrogéomorphologique, qui inondent complètement la surface des plaines alluviales, est en général possible (profil en travers topographique de la plaine alluviale de forme concave) à l'aide de modélisation hydraulique par tronçon homogène. Les résultats de travaux 
universitaires (Chave, 2002 ; Esposito, 2003) et d'études techniques portant sur ces mêmes crues et inondations récentes montrent que les valeurs estimées des débits hydrogéomorphologiques sont supérieures ou égales aux valeurs estimées des débits des crues historiques et des débits de fréquence centennale.

Une approche complémentaire, appelée paléo-hydrologie, permet par ailleurs la reconstitution du niveau atteint par les plus fortes crues depuis plusieurs millénaires, en s'intéressant aux dépôts laissés par les crues, soit directement dans le champ d'inondation, soit dans des grottes surplombant le lit mineur. Des techniques d'analyse stratigraphique et de datation des dépôts (carbone 14, thermoluminescence, césium 137, plomb 210...) sont alors utilisées pour proposer une chronologie de crues (House et al., 2002 ; Benito et al., 2004). L'estimation des débits de crue est facilitée par le choix de sites où les variations d'altitude du fond du lit peuvent être considérées comme faibles à l'échelle de quelques milliers d'années. Les résultats obtenus sur le bassin de l'Ardèche (Sheffer et al., 2003a) ont montré que la crue de 1890, la plus forte connue sur une période historique de quatre siècles, n'avait pas été dépassée depuis plusieurs milliers d'années. Inversement, les analyses paléo-hydrologiques menées sur deux grottes situées dans les gorges du Gard (Sheffer et al., 2003b) ont mis en évidence la trace de cinq crues ayant dépassé le niveau de la crue de septembre 2002, dont trois sur la période 1400-1800.

Ces deux approches naturalistes (hydro-géomorphologie, paléo-hydrologie) sont intéressantes dans la mesure où elles permettent d'élargir de façon considérable l'échelle chronologique d'investigation, et de donner des éléments objectifs sur les crues majeures survenues sur le bassin versant. Elles soulèvent toutefois des difficultés d'interprétation pour déterminer si les niveaux de crues atteints dans les conditions climatiques et géomorphologiques du passé restent représentatifs du risque d'inondation futur (variations climatiques à l'échelle pluriséculaire, divagation latérale du cours d'eau dans la plaine d'inondation, changement de l'occupation du sol sur le bassin).

\subsection{4 Études hydrologiques antérieures}

II est nécessaire de faire la synthèse des études déjà réalisées au droit du site et sur le bassin versant. Outre les estimations des crues de référence, on note la méthode d'extrapolation utilisée, le jeu de données exploité (période de mesure considérée), le recensement des données, la critique des données, et toutes remarques et informations sur le régime des crues et les conditions d'écoulement propres au bassin.

\subsubsection{Mode de fonctionnement du bassin en crue}

Il est également nécessaire de faire la synthèse des éléments disponibles permettant d'apprécier le mode de fonctionnement du bassin pour les plus forts événements de crue : description-typologie météorologique, répartition spatiale des précipitations, dates dans l'année, cotes atteintes et zones de fortes vitesses, genèse des crues (pluviale-nivale), conditions particulières d'écoulement (présence de champ d'inondation, transport solide et changements morphologiques du lit mineur et/ou du lit majeur, endiguements, brèches/ruptures de digues ...).

\subsection{Prédétermination des crues extrêmes sur un site jaugé}

Les méthodes basées sur l'ajustement local de séries de débit présentent les limites décrites en section 2.1, et c'est pourquoi on doit avoir recours aux autres méthodes décrites dans 
cette section 2.4, soit en augmentant la taille de l'échantillon des débits, soit en conditionnant l'extrapolation de la distribution des crues à une connaissance sur la distribution des pluies (Lang et Lavabre, 2007).

\subsubsection{Méthodes basées sur les débits}

\subsubsection{Théorie des valeurs extrêmes appliquée à des jeux régionaux}

Une première façon d'augmenter la taille de l'échantillon consiste à élargir le domaine spatial d'observation et à analyser simultanément les observations de différents postes de mesure d'une zone supposée homogène. La première application (Dalrymple, 1960) considérait que les distributions des sites d'une même région homogène étaient identiques, à un facteur multiplicatif près, appelé indice de crue. De nombreux raffinements ont été apportés depuis (Hosking et Wallis, 1997a, St-Hilaire et al., 2003, Ribatet et al., 2006), notamment sur la notion d'homogénéité hydrologique (découpage géographique, puis voisinage hydrologique) et sur le mode de traitement statistique qui permet de combiner information locale et régionale, avec un poids fonction du degré d'homogénéité-hétérogénéité du jeu régional de données. Une des limitations de ces approches est souvent de ne pas considérer l'impact de la dépendance spatiale entre données, qui peut conduire à sous-estimer l'incertitude finale sur l'estimation des quantiles de pluie et de débit de référence (Hosking et Wallis, 1997b).

\subsubsection{Théorie des valeurs extrêmes appliquée à un échantillon mixte (crues du réseau d'observation systématique et crues historiques)}

Les sociétés humaines ont été confrontées au risque d'inondation dès lors qu'elles se sont installées aux abords des cours d'eau. Par conséquent, les crues les plus catastrophiques des siècles passés ont laissé des traces, sous formes de témoignages, de descriptions plus ou moins exhaustives du phénomène voire parfois de relevés quantitatifs. L'intégration de données historiques vise à prendre en compte ces informations relatives aux événements les plus catastrophiques (et donc les plus rares) dans le calcul des quantiles de crues. L'intérêt est évidemment d'élargir l'échelle temporelle d'analyse, en utilisant des événements exceptionnels, qui sont potentiellement les plus riches d'information pour l'estimation des quantiles extrêmes. Par contre, les données issues d'enquêtes historiques sont entachées d'incertitudes beaucoup plus importantes que les données récentes issues de mesures continues.

$\mathrm{Au}$ niveau méthodologique, cette approche réclame une collaboration entre historiens et hydrologues. Le travail de l'historien consiste à identifier les sources potentielles d'information, puis à effectuer des recherches dans les archives afin de recenser les événements passés et les données associées. Un des points importants de cette analyse est d'assurer l'exhaustivité du recensement, c'est-à-dire d'assurer qu'aucun événement supérieur à un certain seuil de perception n'a pu être oublié. Le travail de l'hydrologue consiste à traduire ces informations en données utilisables pour les calculs de prédétermination, puis d'estimer les quantiles en utilisant des modèles statistiques adaptés aux particularités des données historiques.

Les données anciennes doivent être traitées comme des données censurées (Naulet et al., 2005) : en effet, la seule information disponible pour la plupart des années anciennes est de la forme «le débit maximal annuel n'a pas dépassé un certain seuil de perception ». De plus, les débits historiques ayant dépassé le seuil de perception sont entachés de fortes incertitudes, pour les raisons suivantes: 
- l'enquête historique fournit généralement des renseignements sur la hauteur maximale atteinte au cours de la crue. Chaque valeur de hauteur est entachée d'une erreur potentielle dépendant de l'origine de la donnée (laisse de crue, observation à l'échelle à un instant précis de la crue, etc.). On se méfiera des changements de système de référence altimétrique (déplacement d'échelles), source d'erreur systématique ;

- les hauteurs sont transformées en débit via un modèle hydraulique. Ceci induit une erreur potentielle importante, liée au calage des paramètres du modèle et aux incertitudes sur la topographie de la section modélisée. À l'inverse des erreurs affectant les hauteurs, ces erreurs de modélisation sont systématiques (c'est-à-dire qu'une même erreur affecte tous les débits reconstitués par un même modèle hydraulique).

Au final, le traitement mixte d'informations sur les crues récentes observées via le réseau de stations hydrométriques et sur les crues historiques des deux à trois derniers siècles permet généralement d'affiner l'estimation des crues extrêmes (Miquel, 1984 ; Parent et Bernier, 2003). Un traitement statistique complet (Neppel et al., 2010), intégrant les erreurs sur les hauteurs et débits des crues historiques, montre toutefois que ce constat est à nuancer suivant la qualité de la reconstitution des crues anciennes.

\subsubsection{Méthodes basées sur la modélisation des processus de transformation pluie-débit}

\subsubsection{Modèles hydrologiques déterministes}

Cette approche consiste à transformer une pluie de référence en crue de référence, en utilisant un modèle hydrologique de transformation de la pluie en débit, adapté aux caractéristiques physiques du bassin versant.

La pluie est représentée par un hyétogramme. Le fonctionnement du bassin versant est déterminé par des paramètres synthétiques qui décrivent les processus d'infiltration, de ruissellement et éventuellement d'évaporation, d'écoulement souterrain et de fonte des neiges.

Le modèle hydrologique le plus fréquemment utilisé est l'hydrogramme unitaire du Soil Conservation Service (Ven Te Chow, 1964), qui décrit le fonctionnement du bassin versant par deux paramètres synthétiques : le temps de concentration et le coefficient CN (« curve number »), associés respectivement à la cinétique de ruissellement et à la capacité d'infiltration.

Il existe de nombreux autres modèles déterministes, parmi lesquels GR4, Topmodel, SWMM, ... Les différents modèles hydrologiques ont été développés et calés sur une certaine typologie de bassins versants, et ne sont donc pas nécessairement bien adaptés pour décrire toutes les situations. II est important que le modèle hydrologique soit bien calé sur des événements réels, pour calibrer correctement les paramètres.

L'intérêt de cette approche est de pouvoir spécifier dans le détail la forme de la pluie de projet et de décrire le processus de rétention en eau du bassin par un modèle hydrologique. La difficulté réside dans le choix adapté des conditions initiales de saturation du bassin qui permette d'attribuer une période de retour à la crue de référence. Une hypothèse forte de ces approches est la correspondance directe entre une pluie de projet de période de retour donnée avec une crue de projet de même période de retour. 


\subsubsection{Modèles probabilistes pluie-débit}

La méthode du Gradex, développée par EDF (Guillot et Duband, 1967) pour la sécurité des grands barrages, est utilisée couramment en France pour l'estimation des débits de fréquences rare et exceptionnelle. La méthode utilise l'information pluviométrique pour extrapoler la distribution de fréquence des débits. Son succès est en particulier lié à sa (relative) facilité de mise en œuvre qui résulte de la simplification extrême du processus de transformation de la pluie en débit. Elle repose sur trois hypothèses :

- la distribution de fréquence des pluies est à décroissance exponentielle simple ;

- la distribution de rétention en eau du bassin versant ne dépend plus de la valeur de pluie ou débit, au-delà d'une certaine période de retour $T_{g}$;

- la forme générale de la fonction de transfert des crues est invariante avec la fréquence.

Le graphique obtenu, avec au-delà d'une certaine période de retour $T_{g}$ un parallélisme entre la distribution des pluies $P$ et la distribution des débits $Q$ (les deux étant exprimés en lame d'eau précipitée - écoulée sur une même durée), peut laisser croire que le passage de la pluie au débit s'effectue par une règle simple: $Q=P-\mathrm{E}(R)$, où $\mathrm{E}(R)$ est la rétention moyenne.

La méthode du Gradex considère en fait une relation générale $Q=\square(P, R)$, où au niveau événementiel une pluie de période de retour donnée $T_{1}$ donne une crue de période de retour $T_{2}$, fonction de l'état initial de saturation : $T_{1}<T_{2}$ si le bassin est déjà bien saturé, $T_{1}=T_{2}$ en cas de rétention moyenne, $T_{1}>T_{2}$ si le bassin a une forte capacité d'interception. L'hypothèse faite sur le comportement de la distribution asymptotique de la rétention conduit à ce parallélisme entre distributions de pluie et de débit, qui n'est pas valide à l'échelle d'un événement pluie-débit. Garavaglia (2011) a illustré sur un cas simple, où la transformation pluie-débit est modélisée par un modèle à réservoir (débit nul si le volume de la pluie n'excède pas la capacité de rétention du réservoir ; sinon débit égal à la pluie moins la capacité de rétention), le calcul de la distribution du débit, conditionné à l'état initial $R$ du réservoir :

$$
\operatorname{Prob}[Q<q]=\int_{R=0}^{R \max } \operatorname{Pr} o b[Q<q \mid R] f(R) d R
$$

En supposant que la distribution du niveau initial du réservoir est uniforme et avec une distribution exponentielle pour les pluies, on obtient une distribution exponentielle pour les débits, de même paramètre d'échelle.

Deux approches prolongeant la méthode du Gradex sont également utilisées en France :

- la méthode Speed (Cayla, 1990, 1995) prolonge la théorie du Gradex en intégrant des théories complémentaires pour établir les relations probabilistes utilisées. En particulier, la relation intensité-durée-fréquence des pluies de Montana permet une application à de petits bassins versants de temps de concentration inférieur à la journée. La méthode Speed comporte deux parties :

- une analyse probabiliste régionale des pluies journalières, fondée strictement sur les axiomes du processus de Poisson et qui, sous réserve de quelques hypothèses supplémentaires de stationnarité spatiale, permet deux améliorations par rapport au calcul statistique : une réduction des incertitudes d'échantillonnage et la mise en évidence (et la quantification probabiliste) de régimes complexes ou de horsains,

- une analyse de la corrélation probabiliste entre pluies journalières et débits de pointe de crue, qui permet de déterminer la relation entre les lois de probabilité de ces deux grandeurs. De cette analyse découle directement la distribution de la pointe de crue, à partir de la distribution des pluies journalières et d'une 
estimation de la capacité moyenne d'infiltration du bassin versant, en mm (à noter qu'il existe un lien entre cette capacité moyenne d'infiltration et la nature géologique du bassin versant, ce qui permet de réduire l'incertitude) ;

- le modèle Agregee (Margoum et al., 1994), qui assouplit les hypothèses de base de la méthode du Gradex, en autorisant pour la pluie des lois non strictement à décroissance exponentielle et en introduisant une fonction analytique pour raccorder progressivement le domaine des crues observées et celui des crues exceptionnelles (saturation progressive du bassin versant).

Par rapport aux approches déterministes, la pluie est ici représentée de façon succincte, uniquement en terme de distribution de volume de pluie et pas en terme événementiel sous forme de hyétogramme. Le processus de transformation pluie-débit reste très sommaire comparé à celui qui peut être introduit dans un modèle pluie-débit. L'intérêt de ces approches est de pouvoir conditionner l'extrapolation de la distribution des crues avec le paramètre définissant la forme de la distribution des pluies fortes.

\subsubsection{Couplage d'un simulateur d'averses et d'un modèle hydrologique}

Des modèles hydrologiques plus complets ont été introduits, à partir de générateur d'averses pour mieux apprécier l'aléa pluviométrique et de modèles pluie-débit pour mieux représenter la transformation pluie-débit.

Le modèle Shypre (Cernesson et al., 1996 ; Arnaud et Lavabre, 2000 et 2002), acronyme de Simulation d'hydrogrammes pour la prédétermination des crues, permet de générer de très longues chroniques de pluies horaires (sur 100000 ans, par exemple) dont on déduit les hydrogrammes de crue par application d'une modélisation simple de la pluie en débit. Le classement des pluies et des débits simulés permet de tracer les distributions de fréquence " empirique » des pluies et des débits de toutes durées, et d'en déduire les quantiles pour une large gamme de périodes de retour, de 2 à 1000 ans, sans faire appel à ce stade à une loi de probabilité. Le progiciel Gershypre permet d'effectuer l'ensemble des opérations : établissement des couples averses-crues, calage du modèle de simulation des pluies horaires, calage du modèle pluie-débit, simulation des hyétogrammes horaires et des hydrogrammes de crue, échantillonnage des pluies et des débits et tracé des distributions de fréquence.

La méthode Schadex (Paquet et al., 2006) estime l'aléa de crue en composant les aléas pluie et état hydrique du bassin. Pour une saison donnée, les pluies sont probabilisées au moyen de lois exponentielles calées sur les sous-populations correspondant à huit types de temps, recombinées avec la probabilité relative d'occurrence de ces types de temps. Une méthode simplifiée de génération d'averses permet de croiser quasi exhaustivement les aléas précipitations et état hydrique du bassin en exploitant une chronique climatologique continue enregistrée sur le bassin. La réponse de celui-ci aux pluies simulées est obtenue à l'aide d'un modèle hydrologique (modèle MORDOR pluie-température-débit, prenant en compte la composante nivale), et conduit à la probabilisation des écoulements simulés et à la détermination de la distribution des débits de crue.

\subsection{Méthodes de transfert vers un site non jaugé}

Bien qu'il existe en France près de 3000 chroniques de débits, la plupart du temps l'hydrologue ne dispose pas d'information hydrométrique directement exploitable sur le site où il désire estimer un débit de référence. 
L'information sur les bassins versants proches doit être alors recherchée. Mais face à l'hétérogénéité spatiale des débits de crue, il est alors préférable de ne pas se limiter à un seul bassin versant voisin mais à en examiner au moins deux ou trois pour tester l'homogénéité ou non des quantiles de débits.

Pour deux bassins homogènes en pluie et en structure, le transfert d'information entre les deux bassins versants, du site mesuré (station) au site à étudier (projet), pourra se faire par exemple grâce à une relation de la forme :

$$
\frac{Q_{\text {projet }}(T)}{Q_{\text {station }}(T)}=\left(\frac{S_{\text {projet }}}{S_{\text {station }}}\right)^{\alpha}
$$

où :

- $Q(T)$ est le débit relatif à la période de retour $T$;

- $S$ la superficie du bassin versant.

Pour les débits de pointe de crue, on peut retenir pour $\square$ une valeur proche de 0,7 à 0,8.

En cas de non-homogénéité (après correction de surface et pluviométrie), des analogies pourront être recherchées selon les caractéristiques physiographiques et géologiques du bassin, l'occupation du sol... La méthode de l'indice de crue (cf. 2.5.2) ou la méthode Shyreg (cf. 2.5.3) permettent une estimation des crues extrêmes sur un site non jaugé quelconque.

\subsubsection{Formules régionales}

Ces formules empiriques régionalisées sont généralement de la forme :

$$
Q(T)=a S^{b}
$$

où :

- $Q(T)$ est le débit $\left(\mathrm{m}^{3} / \mathrm{s}\right)$ de pointe relatif à la période de retour $T$;

- S est la superficie du bassin versant $\left(\mathrm{km}^{2}\right)$;

- $\quad a$ et $b$ sont des coefficients fonction de la période de retour $T$.

Ces formulations découlent de synthèses régionales. Le coefficient a est lié au régime pluviométrique et à l'aptitude au ruissellement du bassin. Le coefficient $b$ est un exposant généralement compris entre 0,7 et 0,8. II tient compte en fait de l'abattement des pluies, ce qui résout implicitement le problème de la dimension spatiale des zones de précipitations intenses, et de l'écrêtement du débit dans le réseau hydrographique.

\subsubsection{Synthèse des débits de période de retour 1000 ans calculés par la méthode du Gradex (Duband et al., 1988)}

La méthode du Gradex a été appliquée par EDF sur de nombreux bassins versants français dont la superficie varie de quelques $\mathrm{km}^{2}$ à quelques milliers de $\mathrm{km}^{2}$. La régression établie sur 170 bassins versants du débit de pointe de période de retour 1000 ans en fonction de la superficie du bassin versant s'écrit :

$$
Q(T)=a S^{0,72}
$$


où $S$ est la surface du bassin versant en $\mathrm{km}^{2}$ et $a$ un paramètre donné par le tableau suivant pour les trois zones suivantes:

- zone I : les bassins affluents de la Loire-Inférieure (Vienne, Creuse...), situés au nord du Massif central, ceux de la Saône, de la Moselle et de la Bretagne ;

- zone II : les bassins des Pyrénées occidentales et centrales, de l'Aude et de l'Ariège, de la Dordogne et du Lot, les bassins de la Durance, du Fier et de l'Arve, des Dranses, de I'Isère ;

- zone III : les bassins de la Haute-Loire, des Cévennes, du Tarn, des affluents rive droite du Rhône à l'aval de Lyon (Eyrieux, Ardèche...), des Alpes-Maritimes, de la Corse.

\begin{tabular}{cccc}
\hline Zone & $a$ & Fourchette à $\mathbf{9 0 \%}$ & Fourchette à 70\% \\
\hline I & 4,05 & $3,07-5,36$ & $3,4-4,8$ \\
\hline II & 7,4 & $5,2-10,4$ & $5,9-9,2$ \\
\hline III & 16,4 & $9,1-29,7$ & $11,3-23,9$ \\
\hline
\end{tabular}

Tableau 2.1 - Paramètre a de la synthèse réalisée

\subsubsection{Formule Crupedix (Diame, 1980-1982)}

$$
Q(10)=S^{0.8}\left(\frac{P J(10)}{80}\right)^{2} R
$$

où $P J(10)$ est la pluie journalière décennale en $\mathrm{mm}$. Le paramètre $R$ varie en France dans la fourchette $[0,1 ; 1,75]$. Cette formule peut être reprise pour une autre période de retour, avec un recalage local du coefficient $R$ pour le jeu de stations jugé représentatif du site cible étudié.

\subsubsection{Méthode de l'indice de crue}

La méthode de l'indice de crue (Dalrymple, 1960) consiste à raisonner sur une distribution régionale obtenue par agglomération des échantillons de crue de plusieurs stations. Pour s'affranchir des phénomènes d'échelle liés à la forte dépendance des crues à la taille du bassin versant, chacune des valeurs $Q_{i}(1)<Q_{i}(2)<\ldots<Q_{i}\left(N_{i}\right)$ d'une station $n^{\circ} i$ est normalisée par l'indice de crue $\mu_{i}=\sum_{j=1}^{N_{i}} Q_{i}(j)$.

Cette méthode de régionalisation peut être utilisée pour estimer les quantiles de débit en site non jaugé, en combinant la loi régionale adimensionnelle (issue de l'agglomération des données de plusieurs sites) et une méthode d'estimation de l'indice de crue (régression multiple à partir de paramètres climatiques ou physiographiques explicatifs).

\subsubsection{Méthode Shyreg}

Cette méthode a été développée pour permettre une première estimation rapide des débits de référence dans le cadre d'élaboration des Plans de Prévention du Risque Inondation. La méthode a été calée sur les quantiles de débits de pointe et journalier des bassins versants jaugés, dans une gamme de superficie de 10 à $1000 \mathrm{~km}^{2}$. Son domaine d'application doit être limité à cette gamme de superficies et, bien sûr, aux cours d'eau qui ne présentent pas 
de spécificités hydrologiques et hydrauliques marquées : bassins excessivement karstiques, bassins avec des champs d'inondation importants... Shyreg est une application régionale de la méthode Shypre, qui associe un générateur de pluies horaires et une modélisation de la pluie en débit pour générer des scénarios de crue. Le développement de Shyreg a nécessité la régionalisation du générateur de pluies horaires et une approche régionale de la modélisation de la pluie en débit. Shyreg aboutit à l'élaboration de bases de données pixelisées au $\mathrm{km}^{2}$ des pluies et des débits qui sont utilisées pour estimer, en tout point du réseau hydrographique, les débits de différentes durées et de différentes fréquences. Un logiciel a été développé, PRD'crue, pour exploiter les bases de données générées par l'application Shyreg. La figure 2.1 donne un exemple de cartes de quantiles de débits sur la zone méditerranéenne.

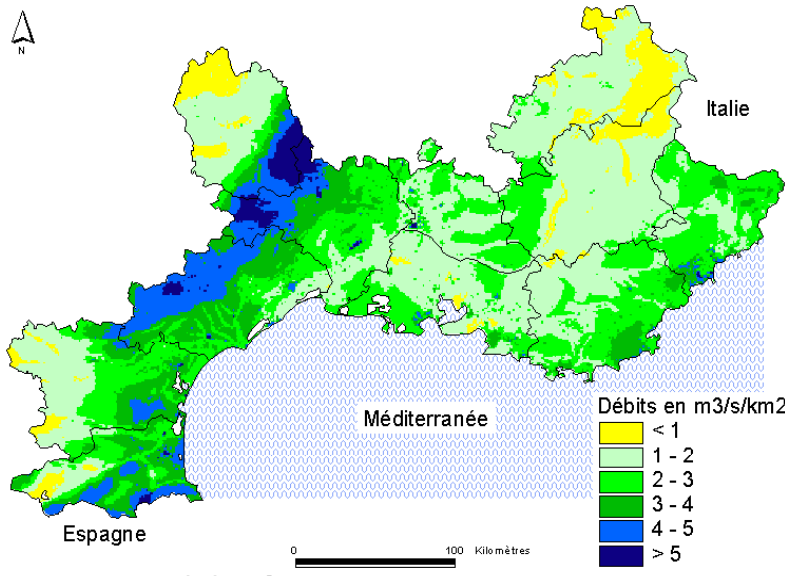

(a) débit de pointe de crue

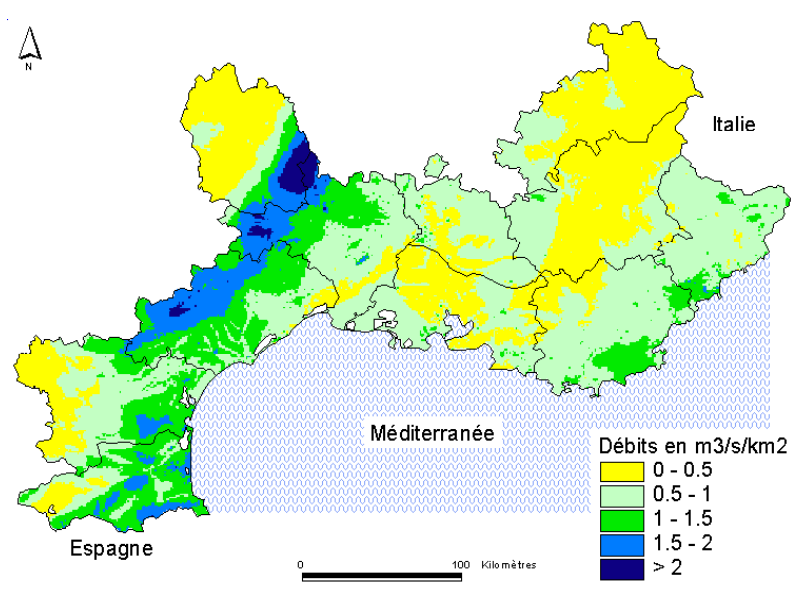

(b) débit journalier

Figure 2.1 - Cartographie par le modèle Shyreg des quantiles décennaux de la zone méditerranéenne française

\subsection{Pluie Maximale Probable / Crue Maximale Probable}

Dans plusieurs pays (en particulier dans les pays anglo-saxons), la méthode Pluie Maximale Probable - Crue Maximale Probable (PMP - CMP), développée par l'école Nord-Américaine, est d'usage courant, sous l'appellation PMP/PMF (probable maximum precipitation / probable maximum flood). Cette méthode définit un débit maximum probable pour le bassin versant considéré, qui est le plus fort débit raisonnablement imaginable. Le risque d'occurrence d'un tel débit est en principe infiniment faible, en tout cas non chiffrable.

La détermination de la Crue Maximale Probable (CMP) repose sur la connaissance de la Pluie Maximum Probable (PMP) sur le bassin versant et sur une modélisation pluie-débit pour calculer le débit de pointe associé. Elle aboutit à un hydrogramme de projet.

\subsubsection{Estimation de la PMP}

La PMP est définie comme étant théoriquement la plus forte précipitation d'une durée déterminée qui est physiquement probable sur un site géographique déterminé et à une certaine époque de l'année (CIGB, 1992). Son estimation repose sur l'utilisation de l'ensemble de l'information pluviométrique disponible sur le site étudié et à proximité. La PMP peut être déterminée à l'aide de plusieurs approches. 


\subsubsection{Approche hydro-météorologique}

L'approche hydro-météorologique d'estimation de la PMP fait intervenir une maximisation des précipitations observées durant les averses. Elle requiert l'analyse systématique de toutes les perturbations atmosphériques ayant affecté le bassin versant qui alimente le cours d'eau à l'étude et les bassins adjacents s'ils sont jugés homogènes du point de vue météorologique. La maximisation exige des corrections d'humidité, suivant l'hypothèse de base voulant que pour produire les précipitations maximales, l'averse a dû disposer d'une alimentation maximale en humidité. Elle utilise un facteur qui tient compte du contenu en eau de l'air au moment de l'averse et du contenu maximal en eau de l'air observé au-dessus de la région, à la même époque de l'année, au cours des années antérieures.

Pour chaque épisode de pluie remarquable sélectionné, l'humidité relative, la température de l'air, la vitesse du vent et la pluie journalière sont relevées. On calcule ensuite la teneur en eau de l'atmosphère associée à cet épisode pluvieux d'une part et au point de rosée maximum d'autre part, tout en tenant compte des effets de barrières orographiques. Le rapport de ces teneurs en eau dans l'atmosphère correspond au facteur de maximalisation.

Le «Manual for Estimation of Probable Maximum Precipitation » (WMO, 1986) comporte des tables de quantité d'eau précipitable pour des couches d'atmosphère variant entre $1000 \mathrm{hPa}$, supposée comme la pression atmosphérique au niveau de la mer, et différentes altitudes jusqu'à 17 km.

Un autre facteur de maximisation à cette relation peut intervenir dans l'estimation de la PMP, c'est le ratio entre la vitesse de vent maximale envisageable et la vitesse de vent observée pendant l'averse sélectionnée. Cependant, l'Organisation Mondiale de la Météorologie (1994) recommande de ne considérer le vent que lorsqu'on est assuré d'une relation nette entre la pluie et la vitesse du vent, ce qui n'est pas toujours évident.

Cette approche a été utilisée dans de nombreuses études hydrologiques destinées à la sécurité des barrages. Ce fut le cas notamment du projet de Tekeze en Éthiopie et du projet de Bagatelle à l'île Maurice. Elle présente l'avantage de prendre en compte explicitement la température et son éventuelle évolution, ce qui peut s'avérer fort utile dans un contexte de réchauffement climatique.

\subsubsection{Approches statistiques}

Si seules des données pluviométriques journalières sont disponibles, mais que l'on ne dispose pas de données d'humidité et autres éléments nécessaires à la maximalisation, la PMP peut être estimée à l'aide des méthodes statistiques suivantes :

- la méthode d'Hershfield (1961) ;

- la méthode Lombardi (1988).

La méthode d'Hershfield a près de 50 ans d'existence. Simple d'utilisation, elle est basée sur une relation statistique faisant intervenir la moyenne et l'écart-type des pluies maximales observées sur une durée donnée. Cette relation a été établie à partir de données pluviométriques sur environ 2000 postes aux USA, mais elle est souvent exploitée sur des sites dont la climatologie est bien différente. Elle permet soit la comparaison avec d'autres méthodes d'estimation soit, dans une certaine mesure, la détermination de la PMP si seules les données pluviométriques journalières régionales sont disponibles.

La méthode Lombardi a été présentée lors du seizième congrès des grands barrages à San Francisco en 1988. C'est une méthode statistique fondée sur l'utilisation de distributions 
bornées. À partir d'une première hypothèse sur le couple (Min, Max) de la population associée à l'échantillon considéré (Garros Berthet, 1995 et 1998), des constantes de valeur centrale et de semi-amplitude sont calculées afin de rendre l'échantillon de pluies maximales journalières adimensionnel avant d'effectuer une transformée logarithmique. Les paramètres d'ajustement à une fonction gaussienne sont obtenus par régression linéaire. Le couple (Min, Max) pour lequel il existe souvent un optimum est obtenu par approximations successives.

Cette approche a été appliquée sur un grand nombre de bassins versants européens, africains et asiatiques. Elle présente l'avantage d'être applicable à tous les climats. Elle présente l'inconvénient, comme la méthode de Hershfield, de reposer entièrement sur l'extrapolation statistique d'une série de pluies à des probabilités très petites. L'utilisation de la méthode Lombardi permet d'avoir une estimation complémentaire de la PMP estimée par la méthode Hershfield.

Ces approches, très simplifiées par rapport à la méthodologie standard de la PMP, doivent être utilisées avec circonspection.

\subsubsection{Approche basée sur un scénario hypothétique mais plausible}

Dans certains cas, la détermination de la Crue Maximale Probable (CMP) peut être basée sur l'établissement d'un scénario hypothétique mais jugé possible dans la zone intéressée par le projet.

Il peut s'agir de supposer qu'un scénario hydro-météorologique connu sur un site ou dans une région donnée peut se déplacer jusque dans la zone intéressée par le projet, et de déterminer un niveau de crue correspondant qui sera une valeur de Crue Maximale Probable. Pour la France, ce déplacement peut être la remontée d'un phénomène cévenol plus au nord que ce qui se rencontre habituellement.

\subsubsection{Estimation de la crue maximale probable}

La Crue Maximale Probable se calcule à partir de la précipitation maximale probable ou de la combinaison la plus défavorable des apports maximaux dus à la fonte des neiges et de la pluie maximale probable pendant la période critique de fonte. La transformation de la pluie en débit est réalisée à l'aide de modèles déterministes.

Le passage de la précipitation à la crue s'effectue en deux étapes successives par l'application d'une fonction de rendement puis d'une fonction de transfert. La première permet de passer de la pluie brute à la pluie nette, la seconde traduit l'atténuation et le décalage temporel de la pluie nette correspondant au décalage des flux dans le réseau hydrographique.

L'estimation du débit de pointe de la CMP peut être obtenue à l'aide de la méthode rationnelle (Mulvaney, 1851). Pour la définition des hydrogrammes de crues, on utilise la méthode de transfert Soil Conservation Service permettant de calculer le volume de ruissellement de la crue à partir de l'averse génératrice et d'un index d'aptitude aux crues des sols du bassin (Ven Te Chow, 1964 ; SCS, 1972). La méthode utilise ensuite le concept de l'hydrogramme unitaire pour la détermination de l'hydrogramme de la Crue Maximale Probable. 


\subsection{Propositions pour la prédétermination des crues extrêmes}

Ce panorama des différentes méthodes de prédétermination des crues montre la diversité des approches, avec la possibilité d'estimations parfois très différentes du risque hydrologique en un site donné. La théorie des valeurs extrêmes constitue la base théorique de référence, mais elle nécessite d'incorporer des informations hydrologiques complémentaires aux séries locales de débits issues du réseau hydrométrique. Dans le domaine des crues extrêmes (à partir d'une probabilité annuelle au dépassement de $10^{-3}$, soit une période de retour supérieure à 1000 ans), les approches basées sur la connaissance des processus de transformation pluie-débit nous semblent incontournables, car elles conditionnent l'extrapolation à l'information pluviométrique. L'effet filtre du bassin versant est beaucoup moins fort pour les crues extrêmes, ce qui donne un poids déterminant aux précipitations dans la genèse des crues extrêmes. De plus, l'analyse des pluies extrêmes, si elle reste délicate, est moins complexe que celle des débits. Le champ spatial des précipitations, lié à la climatologie et aux influences orographiques, est plus homogène que celui des débits, très influencé par la structuration du réseau hydrographique et les propriétés des bassins versants (physiographie, géologie, couverture). On dispose par ailleurs d'une information beaucoup plus riche sur la pluviométrie, grâce à une meilleure densité du réseau d'observation et une plus grande antériorité.

La méthode du Gradex a été depuis les années 1970 la référence en France pour l'estimation des crues extrêmes pour le dimensionnement des évacuateurs de crues des grands barrages. Elle a fait l'objet d'une publication technique (CFGB, 1994), avec la présentation des principes de la méthode et plusieurs exemples d'application. Le fondement de l'approche reste aujourd'hui pertinent. La méthode du Gradex et ses prolongements Speed et Agregee continuent à être utilisés aujourd'hui, et par ailleurs les méthodes de simulation de longues séries hydrométriques, à partir du couplage d'un générateur d'averses et d'un modèle hydrologique, ont fait l'objet d'un travail significatif dans la mise au point de la structure des modèles et ont permis des avancées significatives dans la connaissance de l'aléa pluviométrique et de sa transformation en aléa de crues extrêmes. Le modèle Shypre et la méthode Schadex sont les deux représentants actuels de ces nouvelles approches. Ils sont maintenant utilisés en remplacement ou en complément de la méthode du Gradex par quelques organismes disposant de budgets d'étude suffisants, ainsi que de bases de données et des outils de traitement nécessaires, qui sont relativement complexes.

La validation statistique de ces modèles reste délicate pour les événements extrêmes, car ceux-ci sont généralement rarement observés à une échelle locale sur les dernières décennies. Le projet de recherche ExtraFlo (2009-12) vise précisément à mieux connaître les domaines respectifs d'application des principales méthodes de prédétermination des pluies et crues extrêmes en France, dont les approches par simulation. À partir de la constitution de jeux de données de référence (numérisation de longues séries, jeux régionaux), on disposera d'une information abondante qui permettra de porter un diagnostic sur les points forts et les lacunes de chaque approche et leur sensibilité à la densification de l'information.

En l'état actuel des connaissances, les quatre méthodes d'extrapolation basées sur l'information pluviométrique (modèles hydrologiques déterministes, méthode du Gradex et ses prolongements Speed et Agregee, modèle Shypre, méthode du Schadex) peuvent fournir des réponses acceptables à condition de s'assurer que l'hydrologue chargé de l'étude propose une méthodologie claire et utilise une méthode qu'il connaît bien et dont il a l'expérience. 
Les résultats gagnent en fiabilité lorsque :

- la détermination de la pluie de bassin tient compte de l'abattement spatial et de la variabilité spatiale des pluies au sein du bassin versant considéré : prise en compte de la topographie, de l'exposition, et éventuellement découpage en sous-bassins pour un grand bassin (pluie hétérogène et modélisation de la cinétique des crues sur les différents sous-bassins) ;

- l'extrapolation statistique des pluies aux grandes périodes de retour est effectuée sur des échantillons dont on cherche à optimiser l'homogénéité, et en tenant compte d'éventuelles particularités climatiques régionales: analyse de la genèse des plus forts épisodes de pluie connus, sur le bassin versant et dans la région ;

- la transformation de la pluie en débit est conduite en choisissant des modèles adaptés à la typologie du bassin versant, et de préférence calés sur des événements réels. Elle est discutée en fonction des variations de contexte susceptibles d'intervenir lors de la crue : saturation préalable, averses consécutives, concomitance entre crues des affluents, ...;

- l'information historique et régionale est utilisée pour resserrer les marges d'incertitude liées à l'extrapolation, à la fois pour l'évaluation des pluies extrêmes et pour l'analyse des crues les plus fortes ;

- des approches différentes sont croisées pour estimer une crue sur un site donné.

La finesse d'analyse de l'étude hydrologique doit être adaptée aux enjeux de cette étude : classe du barrage et sensibilité du barrage à l'aléa crue.

Dans certains cas, les méthodes classiques doivent faire l'objet d'adaptations :

- la composante nivale doit être intégrée sur les bassins d'altitude ;

- les petits bassins versants (inférieurs à quelques $\mathrm{km}^{2}$ ) posent des difficultés spécifiques: ils sont généralement non jaugés, et les méthodes traditionnelles ne sont pas toujours bien adaptées. II y a lieu de raisonner sur des pluies et crues à pas de temps adaptés (une à quelques heures), capables de représenter l'occurrence d'orages très concentrés. D'autre part, il convient d'utiliser des méthodes de transformation pluie-débit capables de rendre compte de la réactivité de ces bassins, sans que l'on dispose d'éléments de calage ; les méthodes de transformation par modèle hydrologique déterministe sont bien adaptées pour apprécier la pointe de crue sur ces bassins.

Plusieurs points spécifiques au dimensionnement hydrologique des évacuateurs de crues des barrages font l'objet de travaux de recherche et ne sont pas encore stabilisés. Ils sont mentionnés au chapitre 5 et concernent :

- la prise en compte des incertitudes pour le choix de l'aléa hydrologique de référence ;

- la possibilité de différents scénarios de crue (pointes multiples, durées de crue, etc.) ;

- le choix d'un état initial de remplissage du barrage et du volume de la crue de dimensionnement ou de la crue extrême (Carvajal et al., 2009). 


\section{Recommandations pour les dispositions constructives et les modalités de maintenance et d'utilisation des évacuateurs de crues}

\subsection{Démarche globale pour garantir la fiabilité}

La fiabilité des évacuateurs qui doit constituer un objectif majeur pour les maîtres d'ouvrages et les bureaux d'études, repose sur une démarche globale incluant toutes les étapes de la vie du projet :

- l'identification des conditions du milieu naturel et de ses contraintes et risques (topographie, géologie, hydrologie, transport solide, séismes, etc.) ;

- l'identification des risques potentiels induits à l'environnement, aux populations et aux biens en aval ;

- la fixation des objectifs du projet et des modalités d'exploitation répondant à ces objectifs ;

- la conception, qui comprend la définition des dispositions d'ensemble de l'ouvrage, la détermination de scénarios de fonctionnement et de chargement, le dimensionnement des organes utilisés pour le transit des crues et la définition des dispositions constructives ;

- la définition des consignes d'exploitation ;

- l'établissement des spécifications techniques pour la réalisation de l'ouvrage (construction du génie civil, fabrication et installation des équipements) et sa mise en service ;

- la construction, la fabrication et l'installation des équipements ;

- les essais de mise en service ;

- le suivi et l'entretien des ouvrages, ainsi que leurs incidences sur la disponibilité des organes au cours de la vie de l'ouvrage.

Outre le soin apporté aux études, aux travaux et aux essais à chaque étape de la démarche, une cohérence globale est nécessaire entre les différentes étapes, par exemple :

- les spécifications pour la qualité de réalisation des parements bétons des coursiers doivent être en rapport avec les conditions hydrauliques attendues, telles que des écoulements rapides avec risque de cavitation ou la présence de transport solide à fort pouvoir érosif ;

- les scénarios de chargement doivent être cohérents avec les conditions naturelles (hydrologie par exemple) et avec les modalités d'exploitation et les futures consignes d'exploitation.

La démarche peut même être itérative dans certains cas (par exemple, tel scénario de fonctionnement peut s'avérer intenable pour l'ouvrage au vu des études, et ceci peut amener à reconsidérer les dispositions d'ensemble de manière à éliminer ce scénario).

Le choix de l'évacuateur doit également être cohérent avec les incertitudes sur l'hydrologie. Notamment, quand plusieurs solutions sont possibles, il convient de choisir celle qui offre le plus de robustesse, au sens large (fiabilité mais également capacité de composer avec des débits et des volumes supérieurs, cf. 3.2.1). Cette réflexion peut même conduire à modifier le choix du type de barrage.

Les barrages, dans leur conception et leur gestion, relèvent de différents domaines: ouvrages de génie civil, ils doivent respecter les règles constructives en vigueur; situés 
en milieu aquatique, ils ne doivent pas aggraver les conséquences des crues. Ils sont également équipés d'éléments mécaniques dont la manipulation et l'entretien doivent garantir la sécurité de l'ouvrage et de l'exploitant. À ce titre, les barrages s'inscrivent dans différents cadres réglementaires.

Le but général recherché est la fiabilité des systèmes contribuant à assurer la fonction d'évacuation des crues, notamment par l'élimination systématique des modes communs et par l'application du principe de redondance. Cela concerne aussi bien le choix des équipements que leur implantation ou leur capacité. Pour assurer la fiabilité de la conduite, le principe de redondance doit s'appliquer non seulement aux équipements mais aussi aux moyens humains mis en œuvre, tant lors de la conception que de l'exploitation.

Des dispositions particulières devront notamment être adoptées dans les domaines suivants, afin de sécuriser au maximum le fonctionnement des organes de manœuvre :

- équipements électromécaniques, notamment alimentation électrique : cf. 3.3.1 ;

- systèmes de commande des vannes : cf. 3.3 .2 ;

- systèmes d'acquisition des données (niveaux, position des organes) : cf. 3.3.3 ;

- systèmes de surveillance et de conduite des ouvrages : cf. 3.3.4.

Par ailleurs :

- les organes de manœuvre, les systèmes électriques et de contrôle-commande, les accès aux équipements essentiels et de manière générale tous les éléments permettant une intervention sur les organes de sécurité de l'ouvrage, seront installés au-dessus des plus hautes eaux connues ou attendues ;

- la sécurité de manœuvre d'un organe de bouchure peut être augmentée en utilisant deux organes de manœuvre de cette bouchure, conçus et calculés pour compenser la perte du second organe de manœuvre. Ces équipements seront également pourvus de moyens de manœuvre auxiliaires (manuels, thermiques...). Toutefois, ces dispositions n'assurent pas une garantie totale, par exemple en cas de tige de vérin faussée.

Par ailleurs, la sécurité d'exploitation des ouvrages d'évacuation de crues est basée sur les principes suivants :

- la sûreté intrinsèque d'un ouvrage doit pouvoir être assurée par l'exploitant, en autonomie et en local, le cas échéant sans système automatisé à sa disposition (en situation dégradée) ;

- le maintien en condition opérationnelle des installations doit être assuré par une surveillance, des contrôles et des essais périodiques.

Les grilles de protection, si elles s'avèrent nécessaires, doivent être suffisamment éloignées de l'entonnement pour que les vitesses d'approche soient faibles, afin de limiter les pertes de charge induites par l'obstacle qu'elles créent. Par ailleurs, afin d'assurer une alimentation correcte de l'évacuateur, il convient de dimensionner la position du plan de grille et sa surface de passage avec une marge de sécurité : un colmatage de $50 \%$ du panneau de grilles doit permettre d'assurer une débitance égale à celle attendue de l'organe qu'elle protège. 


\subsection{Systèmes concernés}

Les principaux systèmes concernés par la sécurité des évacuateurs sont les suivants :

- les pertuis, qu'ils soient vannés ou non ;

- les vannes ou clapets, que nous désignerons par le terme vannes ;

- les systèmes fusibles ;

- les organes de manœuvre : en général, la manœuvre est assurée par un système mécanique à entraînement électrique ou hydraulique, de type treuil à chaîne ou vérin (la tendance actuelle est de préférer les vérins) ;

- dans le cas le plus fréquent où les vannes sont à commande manuelle ou automatique nécessitant une source d'énergie autre que celle de la retenue, les systèmes concernés incluent l'alimentation électrique et les systèmes contrôle commande ou systèmes de surveillance et de conduite du barrage. Dans les cas où il s'agit de vannes ou clapets automatiques à commande hydraulique à partir de la retenue, les systèmes concernés incluent les flotteurs, les contrepoids, les capteurs...

Les dispositions constructives et modalités de maintenance présentées dans ce chapitre s'appliquent à tous les évacuateurs, y compris les non vannés.

Par ailleurs, il convient d'insister sur le fait que le dimensionnement hydraulique d'un évacuateur de crues, quel que soit son type, doit intégrer l'ensemble de l'ouvrage, notamment à cause de la possible variation de la section de contrôle en fonction du débit, qui peut avoir une influence importante sur la cote amont (cf. 4.3.4).

\subsubsection{Types d'évacuateur}

Il existe deux grands types d'évacuateurs : les évacuateurs de surface et les évacuateurs en charge (de fond ou de demi-fond). Les évacuateurs de surface peuvent être libres ou vannés. Les évacuateurs de fond sont vannés (sauf pour les barrages écrêteurs de crue à pertuis ouvert).

Les évacuateurs de surface ont un débit proportionnel à la lame déversante puissance $3 / 2$ (différence de cote entre le seuil et la retenue loin à l'amont), tant qu'ils restent dénoyés, tandis que les évacuateurs en charge ont un débit proportionnel à la racine carrée de la différence de niveau d'eau (différence mesurée par rapport à la cote de la section de contrôle aval). De ce fait, l'augmentation de débitance relative de ces derniers est beaucoup plus faible lors d'une augmentation de cote, ils ont donc moins de «marge » vis-à-vis du débit des crues.

Cette caractéristique revêt une grande importance, qui peut être illustrée par le schéma suivant (cf. fig. 3.1), synthétisant la marge de manœuvre disponible en cas de sur-niveau :

- évacuateurs en charge : le débit est fonction de la racine de la différence de niveau entre l'amont et l'aval, car l'écoulement est en charge. Par exemple, si le niveau de la retenue monte de $2 \mathrm{~m}$ pour une chute amont-aval de $50 \mathrm{~m}$, la chute augmente de $4 \%$ et le débit augmente de $2 \%$ seulement ;

- évacuateurs de surface : le débit est fonction de la lame déversante puissance 3/2. Par exemple, si la lame déversante passe de 2 à $4 \mathrm{~m}$, le débit augmente de 180\%. 


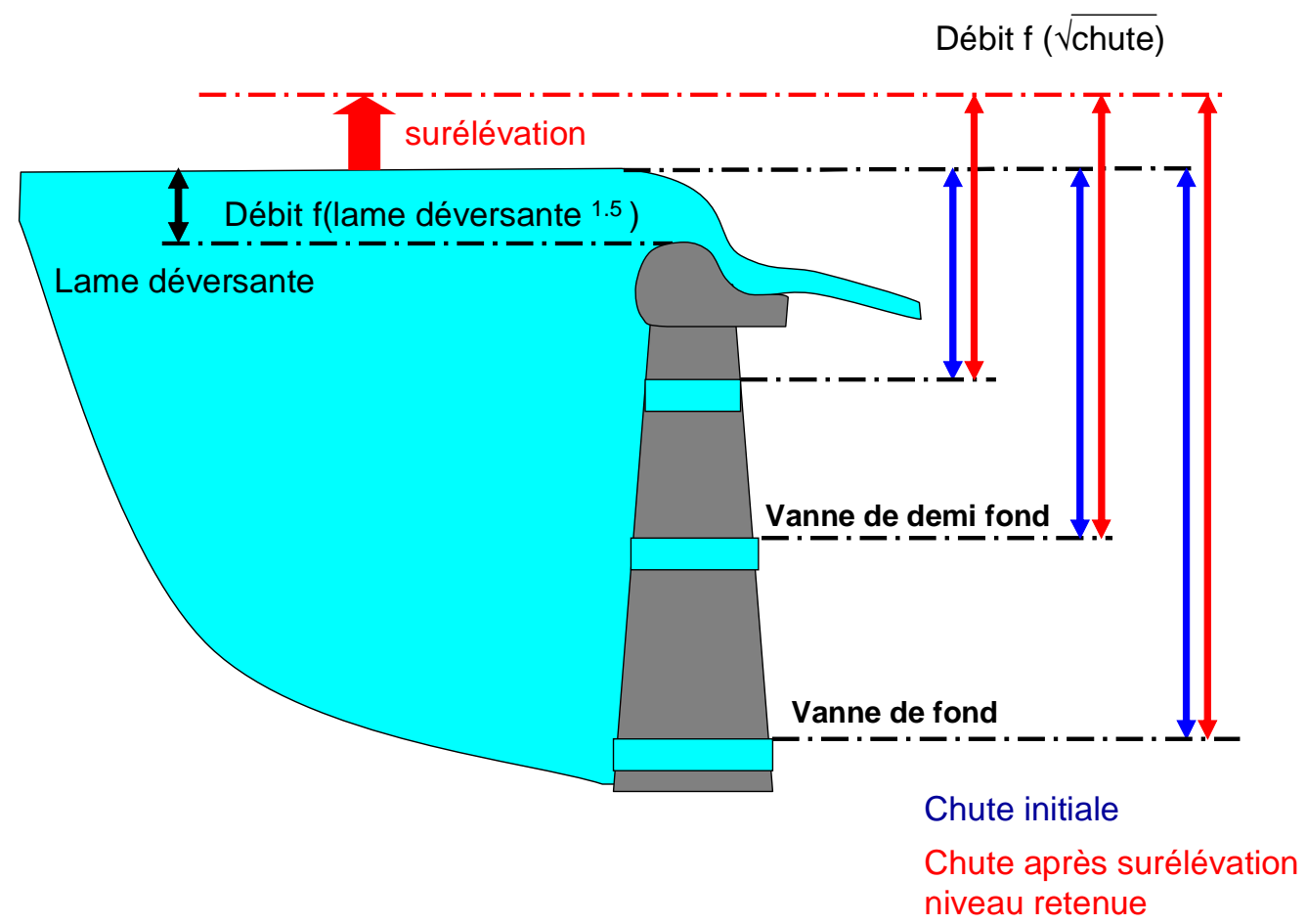

Figure 3.1 - Accroissement de la débitance selon l'organe

Il y a donc une grande différence vis-à-vis des risques entre ces deux types d'évacuateurs en cas de dépassement du débit de la crue exceptionnelle.

On trouve aussi des vannes en position intermédiaire, notamment de demi-fond (cf. fig. 3.1), dont l'accroissement de débitance avec la charge sera meilleur que pour une vanne de fond, mais nettement moins que pour un évacuateur de surface.

Il convient de signaler ici que les évacuateurs en tulipe sont à la lettre des évacuateurs de surface puisque la prise de l'eau se fait en surface. Ils fonctionnent avec un écoulement à surface libre pour les faibles débits. À partir d'un certain débit, l'écoulement n'est plus contrôlé par le seuil, mais par la galerie qui se met en charge. Les tulipes sont donc en pratique à considérer comme les évacuateurs en charge du point de vue de leur loi de débit en crue forte.

Enfin, les évacuateurs de surface à surface libre, peuvent fonctionner en régime noyé audelà d'un certain débit. Leur loi de débit est alors fonction non seulement de la charge amont sur le seuil, mais du niveau à l'aval du seuil. Elle est moins favorable que la loi en puissance $3 / 2$ citée plus haut. Cela concerne aussi bien les évacuateurs à entonnement frontal que ceux à entonnement latéral.

On trouve dans les évacuateurs de surface les évacuateurs à seuil libre, les systèmes fusibles, les systèmes gonflables et certains évacuateurs vannés (pertuis de surface munis de vannes ou de clapets). Les évacuateurs en charge sont évidemment des évacuateurs munis de vannes obturant les pertuis.

Certaines recommandations étrangères proposent d'éviter les évacuateurs de crues vannés lorsque le temps de base de la crue est inférieur à 12 heures et recommandent d'étudier particulièrement cette alternative pour des temps de base entre 12 et 24 heures, en fonction des conditions d'exploitation (USACE - Hydraulic design of spillways). 
Les conditions d'exploitation, de présence ou d'accès de l'exploitant sur le barrage et la cinétique des crues doivent être prises en compte pour le choix et le dimensionnement des matériels voire du type d'évacuateur, vanné ou non vanné. Ces conditions d'exploitation peuvent résulter de la politique propre au maître d'ouvrage, en cohérence avec son modèle d'exploitation et de maintenance, ainsi que des conditions économiques du projet (coût de construction, coût de maintenance, profits d'exploitation).

\subsubsection{1 Évacuateurs à seuil libre (sans organe mobile)}

Les évacuateurs de crues à seuil libre sont naturellement des évacuateurs de surface. Leurs principaux avantages sont les suivants:

- pas d'organes mobiles donc une fiabilité accrue ;

- pas de présence permanente nécessaire sur l'ouvrage, ce qui les rend très intéressants lorsque les crues sont dites « rapides » ou pour des sites isolés ;

- très peu d'entretien.

Il existe divers types de seuils libres, dont la forme hydraulique est plus ou moins complexe et détermine le coefficient de débit de l'ouvrage: seuils profilés, seuils minces ou épais, seuils labyrinthes, seuils à touches de piano (PK-Weir), etc. Le choix du type de seuil dépend de la hauteur d'eau admise pour la surélévation du plan d'eau, du débit à évacuer et de la longueur de seuil disponible. Il résulte le plus souvent d'un calcul technico-économique entre le coût de l'ouvrage et la valorisation de la réserve d'eau.

La hauteur de la lame d'eau doit être étudiée en fonction des corps flottants, afin que ceux-ci ne restent pas coincés sur le seuil par manque de tirant d'eau.

La présence de grilles ou autre barreaudage juste devant ces évacuateurs est fortement déconseillée, car en cas de colmatage, le barrage se retrouve sans organe d'évacuation ou avec une débitance quasi nulle (le risque est évidemment accru lorsqu'il s'agit de grilles à mailles fines). Dans le cas contraire, des précautions doivent être prises (cf. 3.2.2).

Lorsque ces seuils se terminent par une chute libre de la lame déversante, il convient de veiller à la bonne aération de cette lame afin d'éviter la mise en dépression entre cette lame et le corps du barrage, ainsi que des vibrations de lame (mise en place de becs de fractionnement, dispositif d'aération).

Tous les seuils sont dimensionnés pour une hauteur de lame déversante donnée, et en cas d'augmentation de cette lame déversante suite à la révision de la crue exceptionnelle, il convient de vérifier si le profil est toujours adapté et ne risque pas d'engendrer des désordres dans l'ouvrage (vibrations, cavitation, mise en dépression des radiers et coursiers, etc.).

La courbe de débitance (débit en fonction de la cote) de ces évacuateurs doit être établie par le projeteur, mais il faut noter que pour les valeurs de lame déversante inférieures à $10 \mathrm{~cm}$, la précision est très faible et ne permet pas une estimation correcte du débit déversé.

Il convient de vérifier que les vitesses de variation des débits relâchés restent compatibles avec la sécurité des avalisants. En particulier, ces débits sont susceptibles de croître très brutalement si la retenue n'est pas à la cote de RN à l'arrivée de la crue (cf. fig. 3.2). 

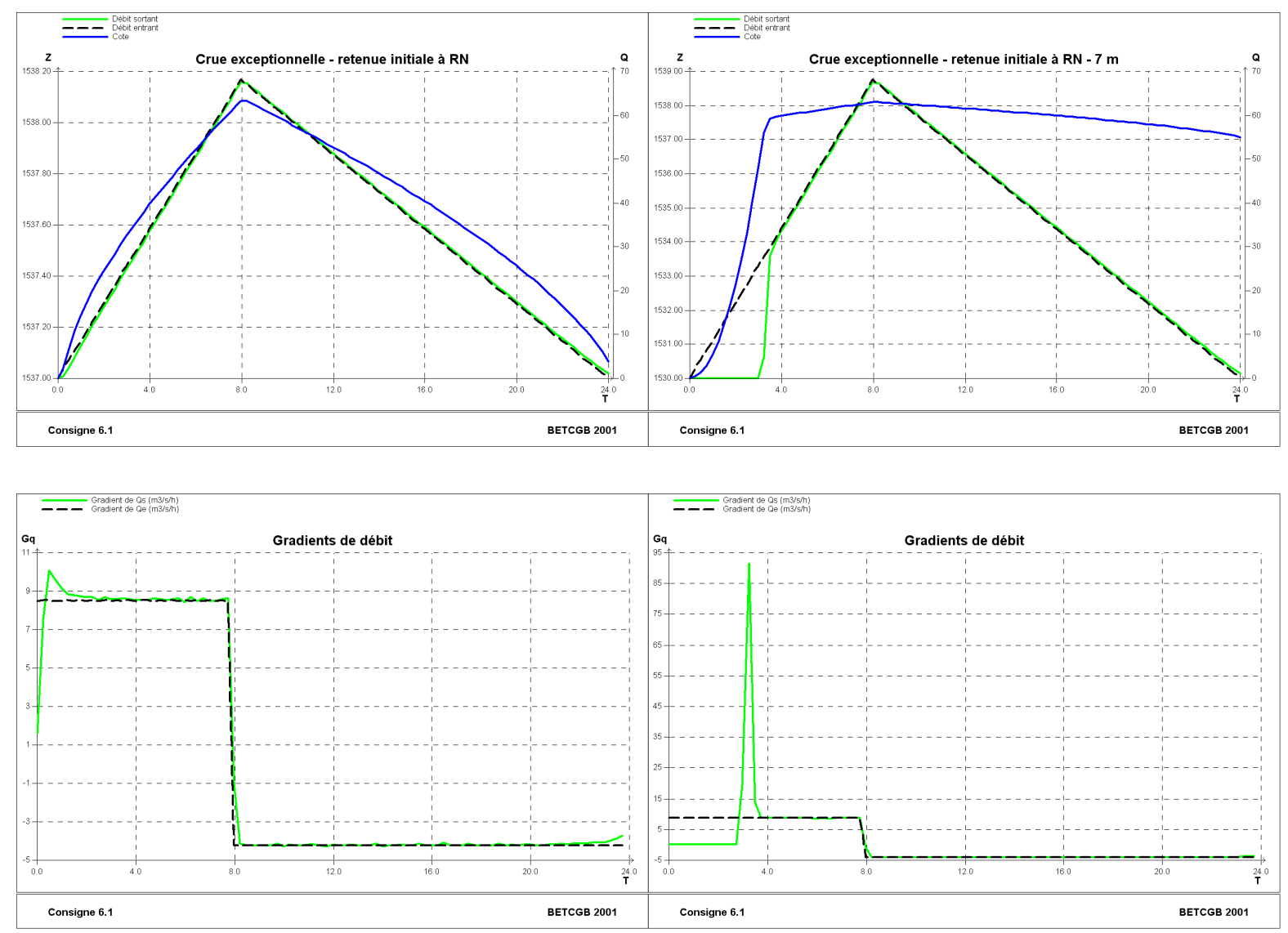

Figure 3.2 - Exemples d'accroissement des débits en fonction de la cote initiale de la retenue (caractéristiques de la retenue et de l'évacuateur identiques)

Certaines dispositions particulières des seuils libres nécessitent des études plus poussées voire des modélisations numériques ou physiques. C'est le cas notamment pour :

- les déversoirs à entonnement latéral dans lesquels l'auge (canal de réception de l'eau en aval du seuil lui-même) a une importance majeure sur la débitance ;

- les tulipes, dans lesquelles le déversoir est en fait une courbe horizontale fermée (souvent un cercle) et où l'eau est évacuée par un puits vertical ou incliné prenant naissance dans la retenue et sortant en aval par un ouvrage en charge ou à surface libre après une section de contrôle. Ces évacuateurs sont sensibles aux corps flottants car le diamètre du puits, ainsi que la section du coude inférieur de raccordement à la galerie d'évacuation ou la section de contrôle de l'écoulement aval, sont souvent trop faibles par rapport aux dimensions d'un arbre avec racines et branches; a minima, il est recommandé d'avoir un diamètre supérieur à $6 \mathrm{~m}$ et une géométrie adaptée au passage des corps flottants (CFGB, 1998).

Par ailleurs, les évacuateurs de surface (déversoirs à entonnement frontal, latéral, en tulipe) présentent l'inconvénient d'être susceptibles de se retrouver en fonctionnement noyé si la lame d'eau devient significativement supérieure à celle prévue pour leur conception, ce qui diminue alors fortement l'accroissement de leur débitance en fonction de la cote (cf. fig. 3.3 et 3.4). Cette particularité doit impérativement être prise en compte lors du dimensionnement de ce type d'évacuateur. 

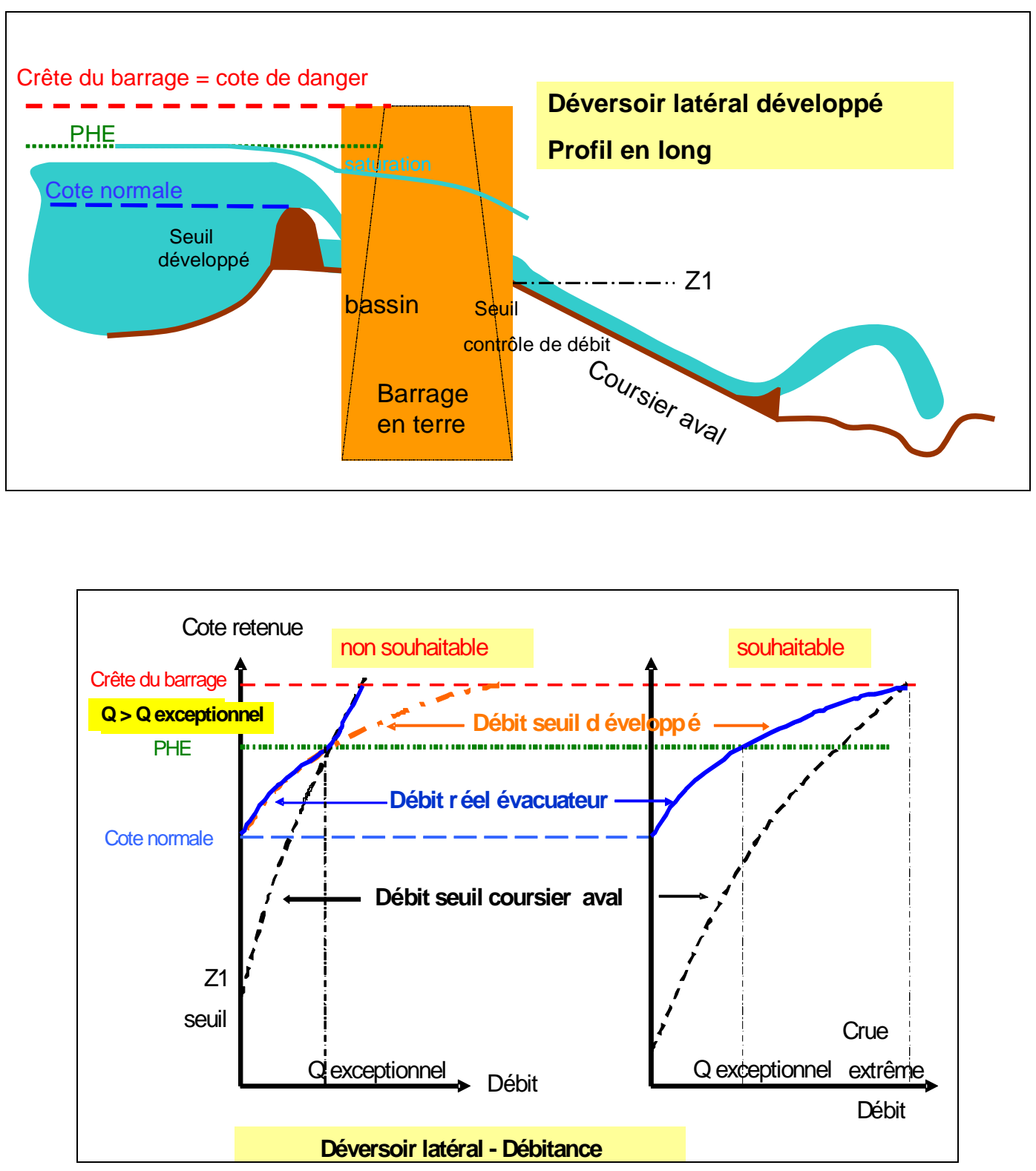

Figure 3.3 - Accroissement de la débitance d'un évacuateur latéral en fonction de la cote, avec Z1 élevée à gauche (ennoiement à partir des PHE), Z1 plus basse à droite (toujours dénoyé) 

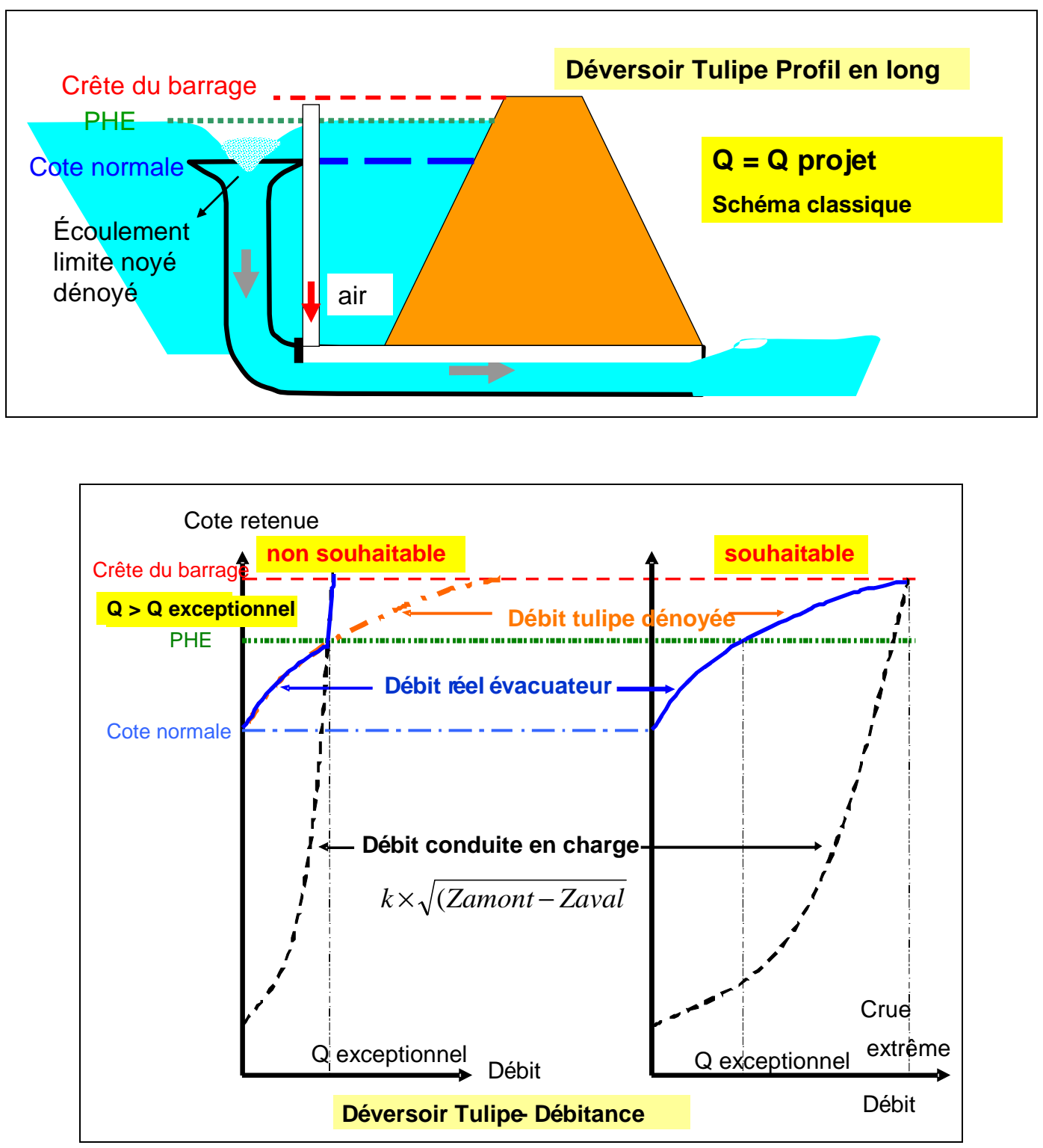

Figure 3.4 - Accroissement de la débitance d'un évacuateur tulipe en fonction de la cote Ennoiement dès PHE à gauche, ennoiement seulement à la cote de danger à droite

\subsubsection{2 Évacuateurs vannés}

Comme indiqué précédemment, les évacuateurs vannés peuvent fonctionner à surface libre ou en charge. Les évacuateurs vannés permettent de contrôler le débit sortant du barrage tout en maintenant la cote amont à une valeur inférieure ou égale à $\mathrm{RN}$, qui ne peut être dépassée tant que toutes les vannes contribuant à l'évacuation des crues ne sont pas totalement ouvertes.

Ainsi, la cote des PHE ne devrait pas, en fonctionnement normal, être atteinte vannes fermées (sauf bien entendu si les cotes de RN et de PHE sont confondues).

Toutefois, les vannes sont sujettes à des défaillances, pouvant remettre en cause la débitance totale de l'ouvrage lors d'une crue. La fiabilité doit donc être un élément prioritaire pour les évacuateurs vannés. 
II est possible d'envisager l'utilisation des vannes de vidange de fond pour l'évacuation des crues. Cela n'est toutefois envisageable qu'à condition que leur sécurité de fonctionnement soit identique à celle des autres évacuateurs de crues, tant du point de vue du fonctionnement hydraulique que du point de vue de l'alimentation électrique, du dispositif de commande et de son accessibilité en toutes conditions. Pour les ouvrages existants, cette possibilité n'est toutefois pas à privilégier en première approche, les installations n'étant généralement pas conçues pour répondre aux exigences des deux fonctions de sécurité (crue, vidange).

La description des différents types d'organes et la conception de leurs systèmes de commande font l'objet de détails dans les paragraphes suivants.

Les évacuateurs de crues vannés de barrages présentent deux caractéristiques spécifiques rendant leur exploitation particulière :

- il n'est pas possible de procéder préalablement à des essais complets de leur fonctionnement en charge ;

- ces organes n'ont a priori aucune position de sécurité évidente vers laquelle il serait possible de se replier systématiquement en cas d'anomalie de fonctionnement; ni la position ouverte, ni la position fermée, ni même le maintien en position ne peuvent jouer ce rôle de sécurité.

Leur conception, leur exploitation et leur maintenance nécessitent un examen au regard des différents risques caractérisant la sûreté hydraulique :

- les risques liés à l'exploitation : la cinétique et l'ampleur d'une ouverture intempestive ou non maîtrisée ont un impact potentiel sur les personnes ou les biens situés à l'aval de l'ouvrage ;

- le risque «crue » : une manœuvre non maîtrisée, la non ouverture ou une ouverture non adéquate présentent un risque pour l'intégrité des ouvrages ou une aggravation des conséquences pour les riverains à l'amont et à l'aval des ouvrages ;

- le risque « rupture d'ouvrage » : une maintenance insuffisante, l'indisponibilité ou le non fonctionnement de l'évacuateur de crues peuvent avoir des répercussions sur la tenue des ouvrages à la suite de l'exhaussement non maîtrisé du niveau de la retenue.

Les cas de défaillance rencontrés invitent à apporter une grande vigilance à la conception des équipements pour éviter les défaillances pouvant se révéler catastrophiques ou plus simplement les complications liées aux modifications à réaliser dans des conditions souvent délicates d'exploitation des ouvrages.

Les défaillances liées aux défauts de conception proviennent fréquemment, non pas de la tenue des structures, dont le calcul est souvent bien maîtrisé, mais des organes de manœuvre, qui sont parfois mal adaptés ou mal calculés en raison des difficultés d'appréhension des efforts et charges «parasites ", liés par exemple aux déformations des équipements et aux efforts de manœuvre. De nombreux cas de défaillances de ce type ont été relevés sur les ouvrages évacuateurs.

Des défaillances liées à un défaut des informations entrant dans le système de gestion sont également rencontrées. Les systèmes de contrôle-commande permettent aujourd'hui une bonne gestion des crues à l'aide d'automates dont il est possible d'assurer la redondance à un coût raisonnable. Cependant, les problèmes les plus fréquemment rencontrés sont liés à une mauvaise information ou, dans certains cas, à un manque d'information fiable en ce qui concerne les débits entrants, surtout en période de fortes crues, du fait de systèmes d'acquisition non fiables. 
Les défaillances liées aux défauts d'entretien sont certainement les plus nombreuses recensées sur les ouvrages hydrauliques. Elles sont le plus souvent sans conséquence grave sur la conduite des équipements. Toutefois, elles peuvent avoir des conséquences dramatiques lorsqu'elles conduisent à la rupture d'un barrage.

\subsubsection{Systèmes fusibles}

Ces systèmes permettent d'augmenter la capacité utile du réservoir, tout en conservant des capacités d'évacuation satisfaisantes. Le système le plus abouti est constitué d'éléments en béton et/ou en acier posés sur un seuil fixe en béton et dont la hauteur peut atteindre plusieurs mètres. La partie supérieure des éléments est généralement en forme de labyrinthe afin d'accroître la longueur déversante avant basculement. Ces éléments sont auto-stables jusqu'à une certaine cote du plan d'eau amont, ce qui permet le passage des crues courantes avec une loi hauteur-débit optimisée, vu le tracé en labyrinthe du seuil. À partir d'une certaine cote, ces éléments basculent automatiquement sous l'effet de la souspression introduite dans une chambre à la base de la hausse par l'intermédiaire d'un puits d'alimentation. Ces puits d'alimentation des chambres sont réglés pour que les hausses basculent de façon séquentielle, au fur et à mesure de la montée du niveau de la retenue, ce qui libère progressivement la section déversante.

Appliqué pour la première fois en France en 1991 (barrage de Lussas en Ardèche), ce système s'est depuis largement développé dans le monde entier, tant sur des ouvrages neufs que sur des ouvrages en service.

Les hausses étant perdues après basculement, l'optimum économique conduit le plus souvent à dimensionner le système pour que le premier basculement se produise pour une crue de période de retour de 50 à 100 ans.

En matière de sécurité, ce système a montré sa fiabilité (risque très faible de basculement intempestif ou de non basculement à la cote prévue), pour autant que des dispositions soient prises pour éviter l'obstruction des puits d'alimentation par les corps flottants (par exemple par installation des puits à l'intérieur des piles ou des bajoyers) et sous réserve d'une surveillance et d'une maintenance adaptées.

Il est également nécessaire d'étudier et de justifier la forme des hydrogrammes sortants, de façon à évaluer les risques liés aux gradients de débit lors du basculement des hausses.

Une autre solution de fusible est basée sur l'utilisation de blocs de béton ordinaire de forme simple en sous-pression permanente, basculant pour une hauteur de lame déversante liée à leur épaisseur. Cette solution est économique mais ne bénéficie pas de l'avantage lié à la forme labyrinthe. Elle est décrite en annexe 3 du bulletin 144 de la CIGB : "Économies dans les barrages » sous le titre « concrete fuse plugs ».

On peut noter également que certains barrages eux-mêmes peuvent être fusibles par conception (cas d'un barrage annexe en terre dans un col, conçu de telle manière que sa rupture sera préalable à celle du barrage principal, cf. 4.3.3.3). Dans ce cas, il ne s'agit cependant pas d'un système d'évacuation à part entière, mais plutôt d'une sécurité supplémentaire s'ajoutant en dernier recours aux ouvrages d'évacuation normaux.

\subsubsection{Systèmes gonflables}

Le principe de base consiste à s'opposer à la poussée de l'eau par une membrane souple fixée sur un radier en béton et gonflée soit à l'air, soit à l'eau. Lorsque le plan d'eau tend à 
s'élever, l'accroissement de la poussée de l'eau dégonfle automatiquement et progressivement la membrane.

Inventé et breveté en 1947 par le professeur français Mesnager, ce procédé a été employé pour la première fois aux États-Unis. Peu développé en France, ce système équipe plusieurs centaines de déversoirs à seuil libre dans certains pays, avec des boudins atteignant des hauteurs jusqu'à plusieurs mètres. Aujourd'hui, les deux systèmes de gonflage à l'eau ou à l'air continuent à coexister, avec chacun leurs avantages respectifs.

L'effet des corps flottants ou des débits solides doit être pris en compte sous la forme de renforcements ou de protections de la membrane.

\subsubsection{Siphons}

Les siphons évacuateurs de crues (cf. fig. 3.5) sont des systèmes qui permettent d'évacuer des débits importants de façon automatique avec de faibles variations de niveau amont. II suffit en général de 2 à $3 \mathrm{~cm}$ pour créer l'amorçage hydraulique d'un siphon et faire passer son débit de quelques $\mathrm{m}^{3} / \mathrm{s}$ à quelques dizaines de $\mathrm{m}^{3} / \mathrm{s}$. Ces dispositifs présentent l'avantage d'une totale autonomie (pas de source d'énergie) et de l'absence de pièce en mouvement.

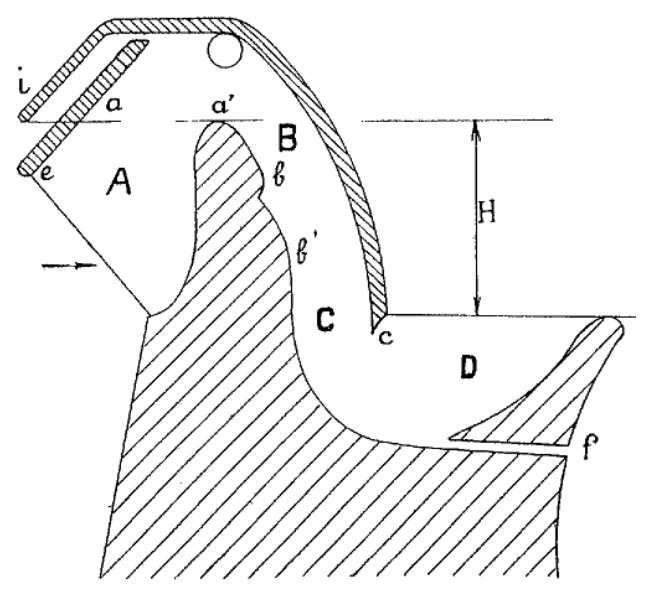

Figure 3.5 - Coupe type d'un siphon

Pour les barrages et en l'absence d'un ouvrage aval de démodulation, il est recommandé de réserver l'usage de ce type d'évacuateur à un fonctionnement au-delà d'une crue de probabilité annuelle de dépassement inférieure à $10^{-2}$ pour les raisons suivantes :

- ils fonctionnent principalement en tout ou rien, et les dispositifs dits de partialisation (fonctionnement à débit partiel par introduction d'air dans le siphon) ont très souvent un fonctionnement aléatoire ;

- ils sont sensibles aux corps flottants qui peuvent en colmater l'entonnement, de section souvent réduite ;

- les efforts sur les parois sont importants : pression atmosphérique d'un côté, dépression de l'autre, avec un maximum au sommet du siphon ;

- l'amorçage et le désamorçage sont rapides et brutaux, ne permettant pas un suivi précis de l'hydrogramme de crue, par l'évacuation de débits intermédiaires (fonctionnement uniquement par paliers). Cette particularité peut entraîner des amorçages et désamorçages successifs pendant la crue, créant un hydrogramme sortant en créneaux ;

- ce fonctionnement en tout ou rien rapide conduit à des gradients de débit aval très élevés, avec les risques associés vis-à-vis des personnes en aval ; 
- vibrations et fatigue du béton.

Aujourd'hui, ce type d'organe est très peu utilisé pour les ouvrages neufs. Leur usage n'est pas recommandé. Certains anciens siphons ont d'ailleurs été démontés et remplacés par des seuils déversants.

À noter que l'on rencontre parfois ces ouvrages comme organes de décharge de canaux : cet usage est acceptable si la restitution des eaux reste contenue dans un bief non accessible aux tiers et prévu pour accepter de fortes variations de débit et de cote.

\subsubsection{Dimensionnement des évacuateurs vis-à-vis des corps flottants}

L'accumulation de corps flottants devant un entonnement crée une perte de charge pouvant altérer significativement le débit de cette passe (évacuateurs vannés, seuils libres surmontés d'un pont ou d'une passerelle, ou évacuateurs suivis d'un puits ou d'une galerie).

Le risque d'obstruction doit être systématiquement pris en compte dans les études, en intégrant l'évolution de l'occupation des sols du bassin versant amont (extension des zones boisées, zones de loisirs aux abords des rivières ou torrents - caravanes, pontons, etc. -, constructions précaires susceptibles d'être emportées par les crues en amont du barrage, zones de dépôts ou de stockage de matériaux ou matériels pouvant flotter, ...).

Les corps flottants peuvent créer des embâcles, du fait d'un tirant d'air insuffisant sous un obstacle ou d'une largeur de passe d'évacuation trop faible, voire par une combinaison de ces deux cas de figure.

La perte de débitance des évacuateurs peut être importante, jusque pour les évacuateurs en puits (tulipes), où le colmatage peut conduire à l'obstruction quasi totale si le diamètre du puits est plus faible que la dimension des corps flottants. Par ailleurs, les évacuateurs en tulipe ont en général des murs anti-vortex qui peuvent faciliter le coincement des corps flottants.

Le dimensionnement des projets d'évacuateurs ou la vérification des évacuateurs existants vis-à-vis de ce risque tient compte des paramètres suivants :

- sensibilité intrinsèque du bassin versant (à la production de corps flottants) ;

- sensibilité intrinsèque de la retenue (configuration de la retenue, orientation des vents, ...);

- sensibilité de l'évacuateur (forme, dispositifs de protection);

- conception de l'évacuateur (largeur des passes, tirant d'air,...).

Ces points sont détaillés dans les paragraphes suivants et un synoptique est proposé en synthèse.

\subsubsection{Sensibilité intrinsèque du bassin versant}

Une étude particulière doit être menée afin de déterminer la propension d'un bassin versant, des rivières amont et de la retenue à produire des corps flottants.

Les corps flottants les plus communément rencontrés sont les débris végétaux naturels, notamment les arbres déracinés par les crues ou lors de bourrasques ou de tempêtes sur l'étendue du bassin versant ou le long des rivières. Des affaissements de berges de la 
retenue peuvent aussi en produire. On notera que ce risque diminue avec l'altitude de la retenue. Au-delà de 1800 mètres, la production de bois devient très faible.

Les activités industrielles ou touristiques peuvent aussi créer des corps flottants: sur les rivières en amont, on identifiera les industries implantées en bord de rivière, telles les scieries, les lieux de campings où des tentes ou caravanes peuvent être emportées, les activités de sport ou de loisirs susceptibles d'en générer (canoë-kayak, hydrospeed,...). En bord de retenue, toutes les activités doivent être recensées : campings là encore, mais aussi toutes les installations légères et équipements de type bungalows, pontons, bateaux, lignes de bouées, corps morts,...

Les corps flottants peuvent être acheminés jusqu'au barrage lors d'événements météorologiques ou de manière différée (les laisses de crues ou les objets flottants peuvent être remobilisés lors d'un événement ultérieur).

L'analyse de risques se fonde à la fois sur le retour d'expérience des situations rencontrées dans le passé et sur la mise à jour régulière d'une base de donnée des activités présentes sur la zone, récentes et/ou intermittentes.

\subsubsection{Sensibilité intrinsèque de la retenue}

La configuration du plan d'eau entre en ligne de compte dans la possibilité de générer une accumulation de corps flottants au droit du barrage. Des vents dominants vers le barrage et un tracé rectiligne de la retenue sont des facteurs aggravants. En revanche, la présence d'anses ou de criques sont susceptibles de stocker ou limiter ce déplacement des corps flottants vers l'ouvrage.

Si pour les barrages dont la retenue est principalement créée par le lit de la rivière, la vitesse d'eau en crue est assez élevée, entraînant vers le barrage la plupart des corps flottants, il n'en est pas de même pour des grandes retenues. À la surface de celles-ci, même en crue, les vitesses sont souvent très faibles sauf à proximité immédiate des déversoirs et des vannes. II y a donc peu d'entraînement d'arbres ou autres débris au travers de la retenue par les courants. Par contre, le vent a souvent une action plus importante sur ces débris, et l'orientation des évacuateurs par rapport aux vents dominants peut avoir une importance majeure.

Pour traduire la capacité d'une retenue à favoriser le transit des corps flottants jusqu'au barrage en situation de crue, il est proposé de comparer le volume des crues de dimensionnement à celui de la retenue. En première approche, on peut considérer comme sensible une retenue dont le rapport de ces deux volumes est supérieur à $30 \%$.

\subsubsection{Sensibilité de l'évacuateur - Système de protection}

Dans le cas où le bassin versant, les rivières amont et la retenue sont susceptibles de produire et d'acheminer des corps flottants jusqu'au barrage, il convient d'examiner la sensibilité de l'évacuateur au regard du risque lié aux embâcles. II peut être envisagé de doter l'évacuateur de crues de dispositifs de protection de l'ouvrage, afin de lui conserver ses performances d'évacuation des débits. En l'absence de tels dispositifs, on retiendra comme sensible un évacuateur dont une réduction de débitance de $30 \%$ conduit à l'atteinte des PHE pour une crue de fréquence centennale. Dans ce cas, il conviendra d'examiner dans le dimensionnement les caractéristiques intrinsèques de l'ouvrage spécifiques au risque d'obstruction - tirant d'air - largeur des passes, exposés en 3.2.2.4 et 3.2.2.5. 
Parmi les systèmes de protection contre les corps flottants, les plus courants sont les dromes, qui sont des barrages flottants souvent constitués de flotteurs reliés par des câbles ou des tiges métalliques. Ils sont maintenus par des ancrages en amont des organes hydrauliques pour intercepter et arrêter les corps flottants et notamment les arbres.

Ils sont fréquemment utilisés pour guider ces corps flottants vers un déversoir de surface et éviter ainsi leur accumulation devant une prise d'eau.

Ils sont parfois utilisés à des fins de protection des organes d'évacuation des crues. Toutefois, cette utilisation requiert des vérifications détaillées de leur résistance et celles des ancrages sous la poussée des corps flottants, voire du gel, car la rupture de la drome provoquerait l'effet contraire, avec un afflux massif et concentré de corps enchevêtrés, créant instantanément un embâcle majeur.

L'impossibilité d'installation ou les inconvénients connus des dromes flottantes ont conduit la CACG à concevoir pour des évacuateurs de type tulipe des systèmes fixes de protection contre les corps flottants, solidaires de l'ouvrage.

Ils comprennent des écrans cylindriques en béton (cf. fig. 3.6) de hauteur suffisante pour être partiellement immergés en partie basse à $R N(-0,50 \mathrm{~m} / \mathrm{RN})$ et hors d'eau en partie haute avec une revanche minimum à la cote des PHE (+0,60 $\mathrm{m}$ au minimum). Ces écrans sont soutenus par des voiles rayonnants encastrés dans la tour de l'évacuateur.

Ils bloquent les corps flottants (avec une tranche "d'eaux mortes ») mais permettent aux " eaux profondes» de passer sous leur base et d'atteindre le déversoir: la vitesse ascensionnelle de l'écoulement reste faible $(0,6$ à $0,7 \mathrm{~m} / \mathrm{s})$ jusqu'à la mise en vitesse à l'amorce du déversement, ce qui annule pratiquement toute perte de charge significative entre la retenue et le plan d'eau en aval des écrans. Les dépressions sur le seuil ne sont que très peu modifiées avec une répartition des vitesses aux abords du seuil quasi inchangée. Secondairement, les voiles supports peuvent avoir un effet anti-vortex non négligeable.

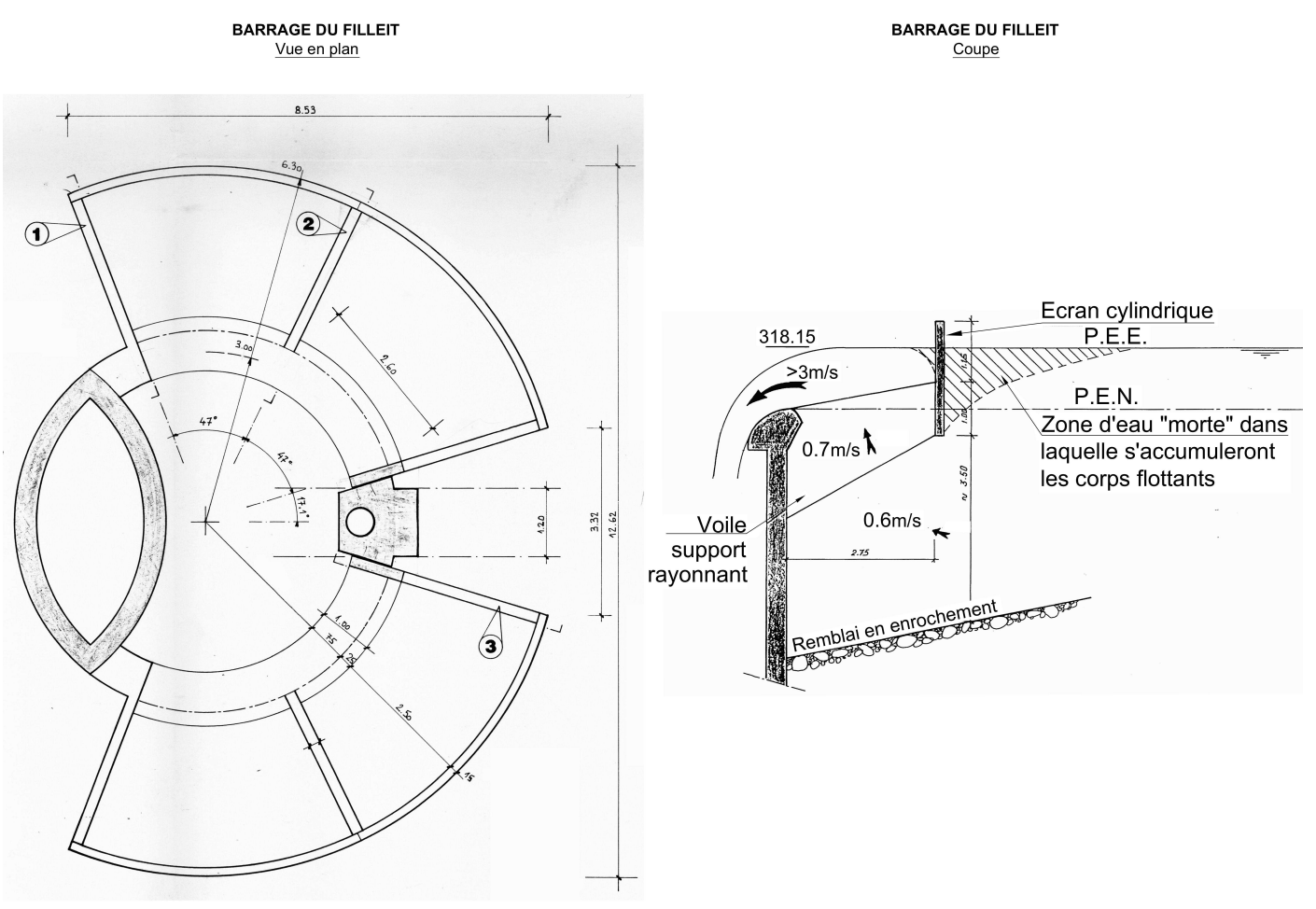

Figure 3.6 - Système CACG de protection contre les corps flottants 
Ce type de dispositifs (cloison siphoïde) est plus généralement adopté à l'amont d'ouvrages lorsque que l'on ne souhaite pas que les corps flottants continuent plus à l'aval.

Dans le même esprit, pour des évacuateurs à seuil libre dont la zone d'approche est peu profonde, on peut installer un peigne à flottants sous forme de poteaux implantés en arc de cercle en amont de l'entonnement (cf. photo 3.1). L'espacement de ces poteaux est en première approche de l'ordre du tiers de la largeur des passes (de façon à ne bloquer que les éléments de plus grande taille, susceptibles de former embâcle). L'ancrage et la section de ces poteaux doivent faire l'objet d'un dimensionnement mécanique pour résister à la poussée des corps flottants qu'ils vont bloquer.

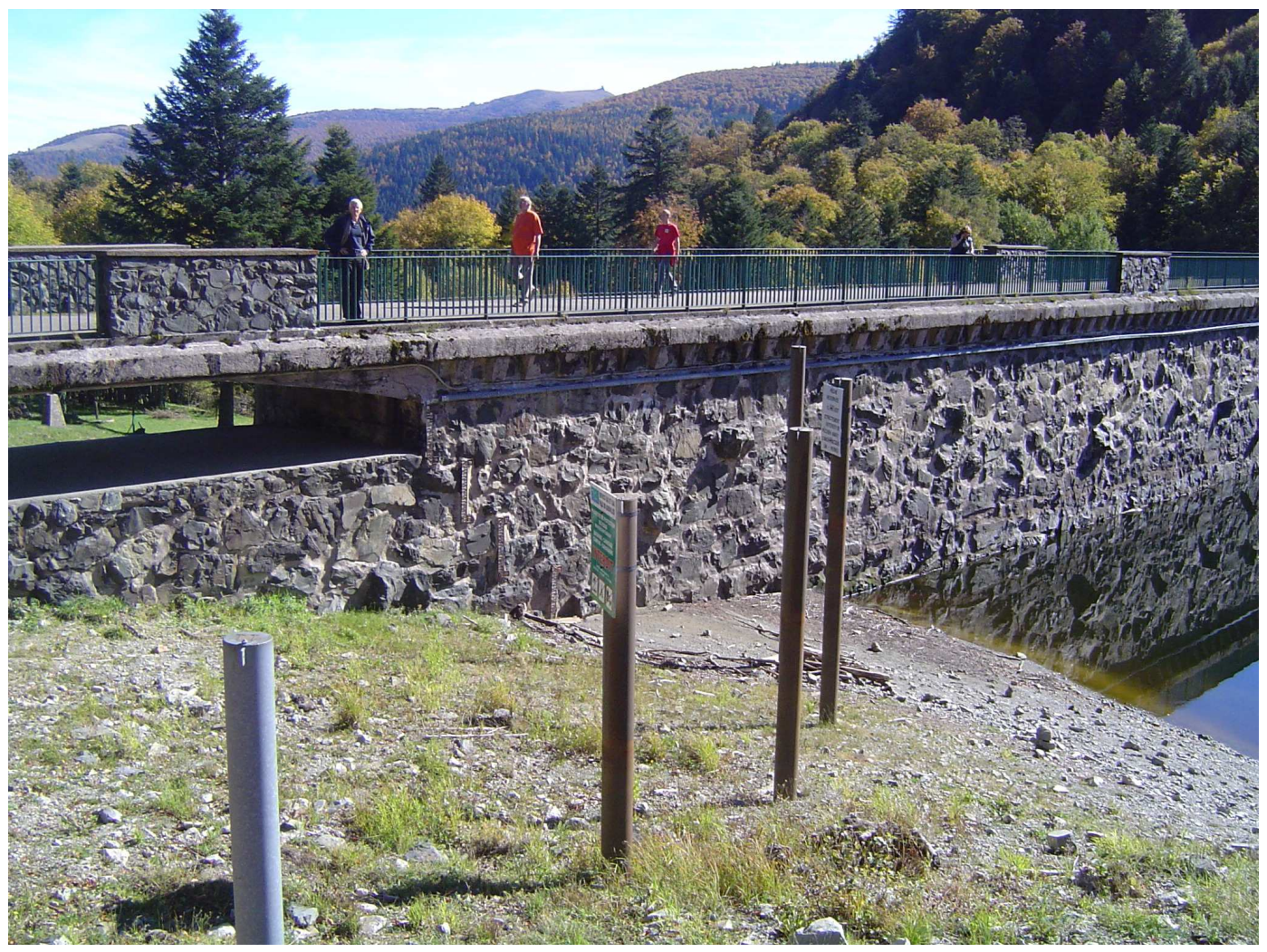

Photo 3.1 - "Peigne à flottants" en amont d'un évacuateur de section restreinte

\subsubsection{Tirant d'air}

Le tirant d'air entre le dessus de la lame déversante et le premier obstacle (couteau de vanne, pont ou passerelle, etc.) doit permettre d'éviter le coincement des corps flottants. Ceux-ci se coinceront d'autant plus que la hauteur de la lame déversante est faible, empêchant les corps de plonger dans la lame d'eau.

Le tirant d'air ne correspond pas à la différence de cote entre le plan d'eau à une cote donnée et le premier obstacle. En effet, la forme de la lame déversante est le plus souvent courbe, ce qui augmente, parfois de façon très importante, la hauteur libre sous cet obstacle. Un calcul hydraulique de la lame d'eau est donc souvent nécessaire.

Dans le cas particulier où l'évacuateur ne présente aucun risque spécifique au regard des corps flottants (pas de production de corps flottants en amont, évacuateur non sensible à l'obstruction), il est proposé de s'assurer d'un tirant d'air minimal de $30 \mathrm{~cm}$. Ce tirant d'air minimal s'applique pour tout écoulement à surface libre sous un obstacle. En effet, le manque de tirant d'air, en l'absence de corps flottants, risque, du fait du batillage de la surface libre, de provoquer un phénomène de mise en charge alternative de l'écoulement, 
induisant une augmentation de la cote amont et des vibrations de la vantellerie et des pièces supports.

Dans tous les autres cas, le CFBR (CFGB, 1997) recommande de laisser un espace suffisant entre le niveau libre de l'eau et la face inférieure du premier obstacle, en moyenne 1,5 à $2 \mathrm{~m}$ suivant l'épaisseur de la lame d'eau pour éviter que les corps flottants ne viennent se coincer : en général, l'obstacle doit être placé en aval du seuil pour les déversoirs libres ou munis de clapets, mais l'encombrement des superstructures sur les ouvrages vannés oblige parfois à reporter ces obstacles à l'amont, ce qui nécessite une rehausse au niveau des piles.

Pour les barrages neufs, on retient ce principe de dimensionnement, pour la crue exceptionnelle (et donc pour une retenue à $\mathrm{PHE}$ ). II est applicable à tout ouvrage au-dessus des écoulements (vannes, ponts et passerelles, ...).

Pour les ouvrages existants, la règle précédente peut être adaptée en fonction du risque réel de transport au travers de la retenue, en tenant compte des obstacles amont (ponts, autre barrage, etc.) et des industries connues.

Dans le cas d'un écoulement libre sur un seuil libre (seuil non vanné ou vannes à pleine ouverture), on doit considérer que les corps flottants ne peuvent passer par-dessus le seuil que si la lame d'eau sur le seuil est supérieure à une lame critique. On devra donc considérer que, tant que la lame d'eau sur les seuils n'est pas supérieure à la lame d'eau critique, une partie au moins des passes peut être bouchée par des corps flottants accumulés sur les seuils, conduisant ainsi à répartir le débit seulement sur les passes opérantes.

En première approche, il est proposé de considérer comme critiques les situations suivantes (pour une crue de dimensionnement) :

- un tirant d'air inférieur à $2 \mathrm{~m}$ si le tirant d'eau est inférieur à $2 \mathrm{~m}$;

- un tirant d'air inférieur à 1,5 m si le tirant d'eau est supérieur à $2 \mathrm{~m}$;

- un tirant d'eau inférieur à $0,5 \mathrm{~m}$.

\subsubsection{Détermination de la largeur des passes}

II est recommandé d'adapter la largeur entre piles (largeur des passes) aux dimensions des arbres susceptibles de transiter. L'ordre de grandeur de la largeur minimale à adopter pour un projet neuf est de 10 à $15 \mathrm{~m}$.

Toutefois, on peut être amené à moduler cette recommandation en fonction de l'altitude : audessus de $1800 \mathrm{~m}$ d'altitude, il y a peu de risque (végétation trop rare). On peut alors réduire nettement la largeur des passes aux environs de $4 \mathrm{~m}$. Au contraire, au-dessous de $600 \mathrm{~m}$ d'altitude, le risque étant accru, il est nécessaire d'envisager une largeur minimale de $15 \mathrm{~m}$.

Cette proposition peut être résumée par la figure 3.7 suivante, pour la France métropolitaine : 


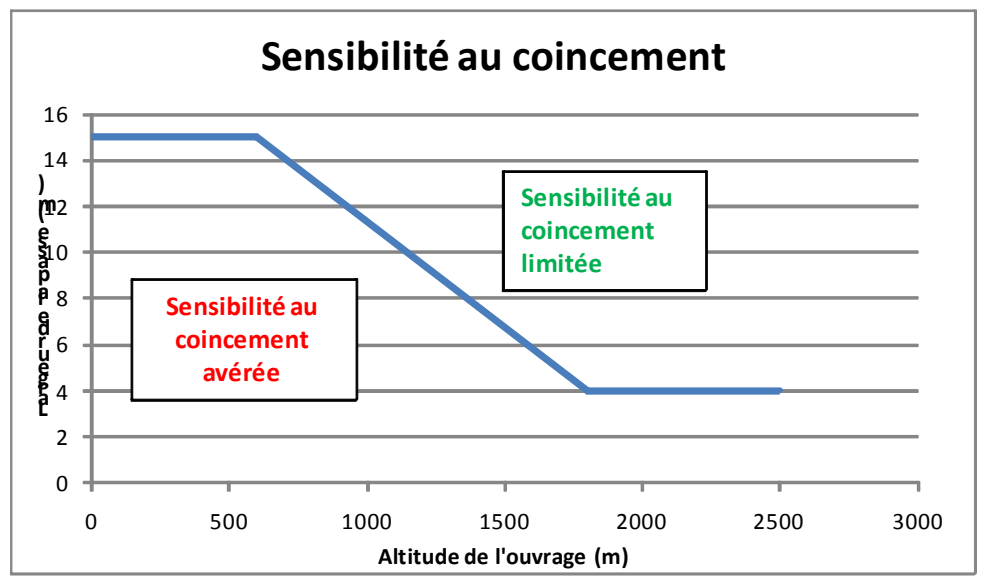

Figure 3.7 - Sensibilité au coincement en fonction de l'altitude

Ces limites pourraient se décaler légèrement vers le haut avec le réchauffement climatique ( $1^{\circ}$ d'augmentation de température entraîne un décal age de $180 \mathrm{~m}$ en altitude des valeurs citées).

\subsubsection{Synthèse}

On propose de considérer le risque embâcles comme non dimensionnant dès lors que ni le bassin versant, ni la retenue, ni l'évacuateur ne sont jugés sensibles. Dès lors, le dimensionnement de l'évacuateur ne répondra qu'aux seules exigences du passage de la crue de dimensionnement (sous réserve d'un tirant d'air minimal de $30 \mathrm{~cm}$ dans ce cas de figure).

Dans tous les autres cas, il est proposé un examen séquentiel de tous les facteurs, pouvant conduire, si le risque persiste après analyse, à tenir compte d'une perte de débitance des pertuis concernés dans le dimensionnement des évacuateurs, ainsi que des efforts supplémentaires générés par ces embâcles sur les structures. (Bister et al., 1994) proposent de prendre en compte une réduction forfaitaire de $30 \%$ de la débitance.

Dans ce cas, les efforts générés par les embâcles sont à prendre en compte. Ces efforts sont horizontaux et appliqués à la cote de PHE, ayant pour valeur la pression exercée par le courant d'eau sur les corps flottants bloqués.

Une étude doit être menée pour déterminer quelle est la population de corps flottants, en particulier les branches ou arbres, qui risquent de se présenter à l'entrée des pertuis de l'évacuateur au moment du passage des crues. Quelles que soient les dimensions des pertuis, on ne peut pas éviter qu'un corps flottant soit bloqué sur le nez des piles. On doit prendre en compte ce cas en supposant un coefficient de contraction supplémentaire à l'écoulement dans la section de contrôle (vanne ou seuil libre) de l'évacuateur de crues.

Un modèle physique peut éventuellement aider à la détermination des conditions d'approche et d'évacuation des corps flottants, pour autant que l'on ait modélisé la retenue suffisamment loin en amont, et que l'on puisse reconstituer sur modèle des corps flottants proches de la réalité.

On notera que la perte de débitance d'une ou plusieurs passes va augmenter fortement l'énergie dissipée à l'aval des passes encore disponibles, qui peuvent subir des affouillements en pied. II convient donc de dimensionner les protections aval en situation dégradée. 
En synthèse, le dimensionnement des évacuateurs au regard du risque lié aux corps flottants est proposé comme suit sur la figure 3.8 (même si bien évidemment la complexité des phénomènes mis en jeu et la variété des situations ne sauraient être réduites à une évaluation par des critères et des valeurs seuils par trop tranchés. Le jugement de l'ingénieur reste et demeure incontournable pour l'appréciation des risques) :

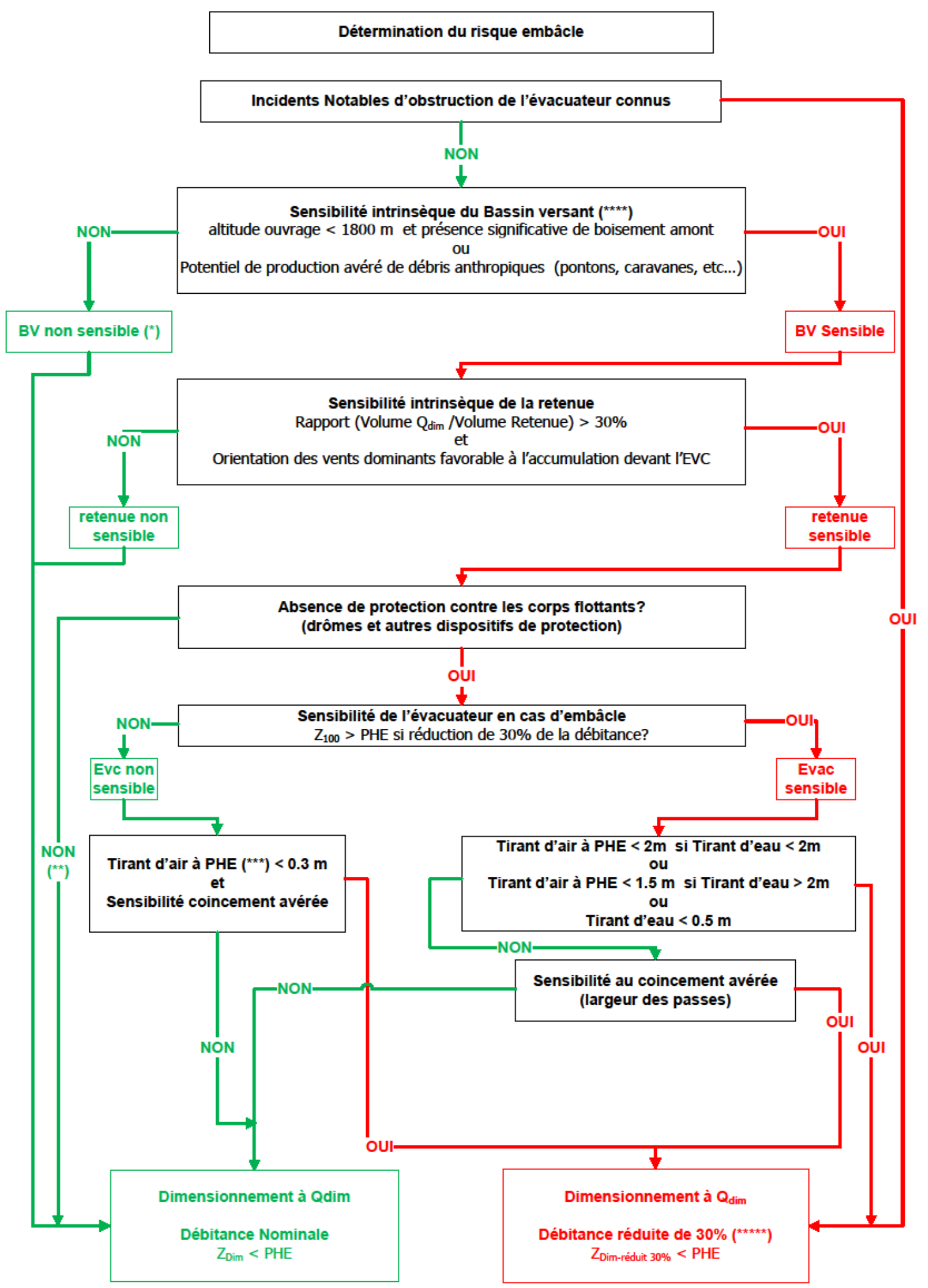

Figure 3.8 - Logigramme de détermination du risque d'embâcles 


\section{Commentaires}

$\left({ }^{\star}\right)$ : on se trouve dans le cas où le bassin versant est jugé non producteur de corps flottants. Les risques de coincement de corps flottants sont donc considérés comme nuls. Les critères de tirant d'air disponible ou de largeur des passes ne sont donc pas déterminants dans la conception de l'évacuateur.

$\left({ }^{* *}\right)$ : on se trouve dans le cas où l'évacuateur est protégé par un artefact contre les corps flottants (drome, masque,...). Les risques de coincement de corps flottants sont donc considérés nuls. Les critères de tirant d'air disponible ou de largeur des passes ne sont donc pas déterminants dans la conception de l'évacuateur.

$\left({ }^{* * *}\right)$ : si le tirant d'air est faible à PHE, la question de la mise en charge du fait du batillage est fondée. Elle ne doit cependant pas être traitée dans cette analyse, qui concerne le risque corps flottants, mais lors de la détermination de la débitance de l'évacuateur qui doit prendre en compte les incertitudes relatives à la cote de mise en charge de l'évacuateur.

$\left({ }^{* * * *}\right)$ : pour limiter les risques d'interprétation variable des recommandations, il faut chercher à objectiver les critères qui permettent de juger de la capacité du bassin versant à produire des corps flottants. Sont donc proposés :

- un critère chiffré sur l'altitude et un taux de boisement ; un boisement " significatif » s'entend comme une présence objective de couverture boisée du bassin, susceptible d'apporter des quantités de débris forestiers (troncs, racines,...) à la retenue. Un taux minimal de boisement de $10 \%$ à $20 \%$ du bassin peut être avancé, mais ne doit pas omettre des situations où l'arrivée de débris végétaux peut être jugée acquise même avec un taux de boisement inférieur ;

- un critère, qui reste malheureusement plus qualitatif, pour tenir compte de la production de corps flottants liée aux activités anthropiques (caravanes, pontons, ...).

$\left({ }^{* * * *}\right)$ : les pertes de débitance à considérer doivent être définies de façon précise. II est suggéré une règle des $30 \%$ excepté pour le cas particulier des tulipes pour lesquelles des pourcentages d'obstruction supérieurs pourront être considérés.

\subsubsection{Coincement des vannes par colmatage du pied des vannes}

L'accumulation des dépôts solides en pied de vannes peut provoquer leur coincement. Ce phénomène peut se produire quelle que soit la nature des dépôts. Dans le cas d'envasement par des matériaux fins, il faut craindre le coincement par effet cumulé de la consolidation des sédiments fortement cohésifs et plastiques et de la poussée de ces matériaux. Pour des matériaux plus graveleux, le coincement est lié à la poussée des sédiments et au frottement important sur le tablier.

Au fil du temps, les matériaux fins déposés dans la retenue se tassent. Par phénomène de consolidation, leur résistance à l'érosion augmente peu à peu ${ }^{5}$. Les matériaux consolidés sont alors très difficilement remobilisables si on a laissé le temps passer. Souvent, ces matériaux sont mêlés à des branches, constituant quasiment de la terre armée, augmentant encore leur résistance. II faut donc impérativement curer régulièrement les matériaux fins déposés en particulier devant les vannes de fond, mécaniquement ou bien en organisant des chasses régulières.

\footnotetext{
${ }^{5}$ la contrainte tractrice critique $\tau_{c,}$ encore appelée rigidité, des matériaux fins $(\mathrm{d}<0,2 \mathrm{~mm}$ ) augmente en fonction de la densité des matériaux à la puissance 4 à 5 , la densité augmentant elle comme le logarithme du temps (cf. travaux de Claude Migniot).
} 
II n'existe pas de dispositif direct de conception susceptible de s'affranchir de ce risque, si ce n'est la création d'obstacles en amont de type seuil batardeau, à condition que ceux-ci soient régulièrement curés en amont pour éviter leur propre engravement. La surveillance et le maintien en conditions opérationnelles des organes de crue sont la garantie du bon fonctionnement de ceux-ci.

La surveillance consiste en un relevé régulier des hauteurs d'envasement/engravement. Ces constats doivent permettre, soit de mettre à jour l'instruction de crue afin de manœuvrer prioritairement les vannes les plus affectées lors de la première crue à venir, soit d'engager un enlèvement mécanique, dès le seuil atteint d'un niveau d'engravement, préalablement estimé ou calculé.

Le niveau d'envasement/engravement peut être contenu par le recours à des essais périodiques de manœuvre des vannes ou par des chasses. II convient cependant de prendre conscience que l'efficacité de ces manœuvres dépend très largement du niveau d'ouverture des organes, de leur durée, de leur fréquence et de l'indice de compacité des matériaux retenus : un cône de sédiments de faible dimension (quelques mètres) peut aisément être dégagé lors de ces manœuvres alors qu'il faudra souvent faire appel à une intervention lourde pour les matériaux restant en place.

\subsubsection{Types de vannes les plus répandus}

Les ouvrages évacuateurs de crues peuvent être constitués de différents types de vannes, en fonction de l'utilisation attendue de l'ouvrage. On peut distinguer les vannes de surface et les vannes de fond.

Les vannes de surface, outre leur utilisation pour contrôler le niveau de retenue dans le fonctionnement courant, assurent la fonction d'évacuation des crues. Ce sont souvent des vannes segments, des vannes wagons ou encore des clapets. On rencontre d'autres types de vannes de surface sur les ouvrages anciens ou s'il existe des contraintes particulières: vannes à rouleaux, vannes bateaux, vannes secteurs, etc.

Ce sont de bons régulateurs de niveau amont, car le débit varie beaucoup en fonction du niveau amont (fonction de la lame déversante à la puissance 3/2).

Les vannes barrant des pertuis en charge (vannes de fond ou de demi-fond) sont rarement choisies pour équiper des évacuateurs de crues. Comme indiqué précédemment, l'inconvénient majeur réside dans la faible variation de la débitance en fonction de la cote de retenue et il convient de prendre en considération l'importance accrue des risques de sédimentation en fond de retenue et donc de blocage des vannages et de colmatage des pertuis. Ce sont principalement des vannes wagons et des vannes segments (cf. 3.2.4.4 également). Les pertuis sont usuellement équipés de deux vannes en série, avec une vanne aval de réglage du débit et une vanne amont dite de garde permettant notamment les opérations d'entretien de la vanne aval. Le dimensionnement de ces vannes (a minima pour la vanne de réglage) doit naturellement permettre la coupure du débit en charge lors de la fermeture de la vanne.

Ce sont de bons régulateurs de débit, car le débit varie peu en fonction des niveaux amont et aval (fonction de la racine de la différence de cote).

Les vannes qui assurent la vidange du réservoir et la chasse des sédiments peuvent éventuellement jouer le rôle d'évacuateur complémentaire sous certaines conditions, avec 
notamment les redondances et dispositifs nécessaires à la fiabilité de fonctionnement de l'organe en situation de crue.

\subsubsection{Seuils réglables}

Les organes suivants sont des seuils dont la cote est réglable, avec déversement à surface libre par-dessus. De ce fait, ce sont de bons régulateurs de niveau amont.

Ils n'ont a priori pas besoin d'énergie pour s'ouvrir, la gravité aidant, ce qui peut représenter un intérêt pour la sécurité. Inversement, ils peuvent aussi s'ouvrir de manière intempestive, ce qui impose des sécurités spécifiques. Ouverts, ils s'effacent dans un logement et ne génèrent pas ou peu de pertes de charges.

\subsection{Clapets}

Les clapets sont des vannes abaissantes constituées d'un bordé généralement de forme cylindrique supporté par une structure en caisson type "ventre de poisson " ou classique articulé sur le seuil du pertuis (cf. fig. 3.9 et photo 3.2).
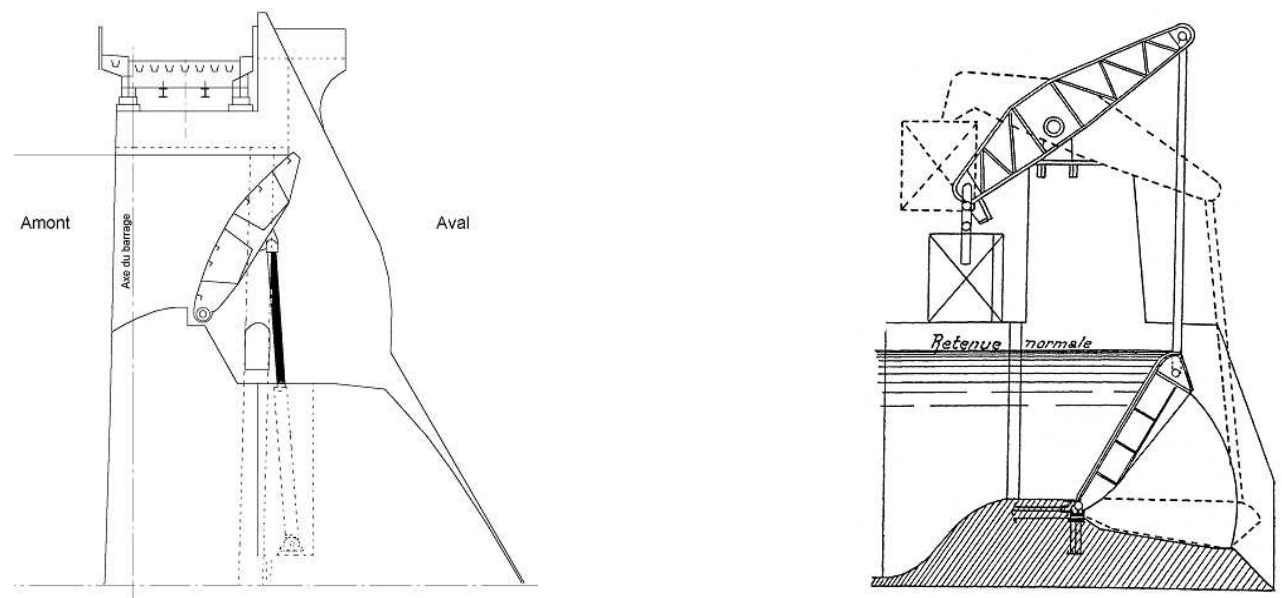

Figure 3.9-Coupes types de clapets

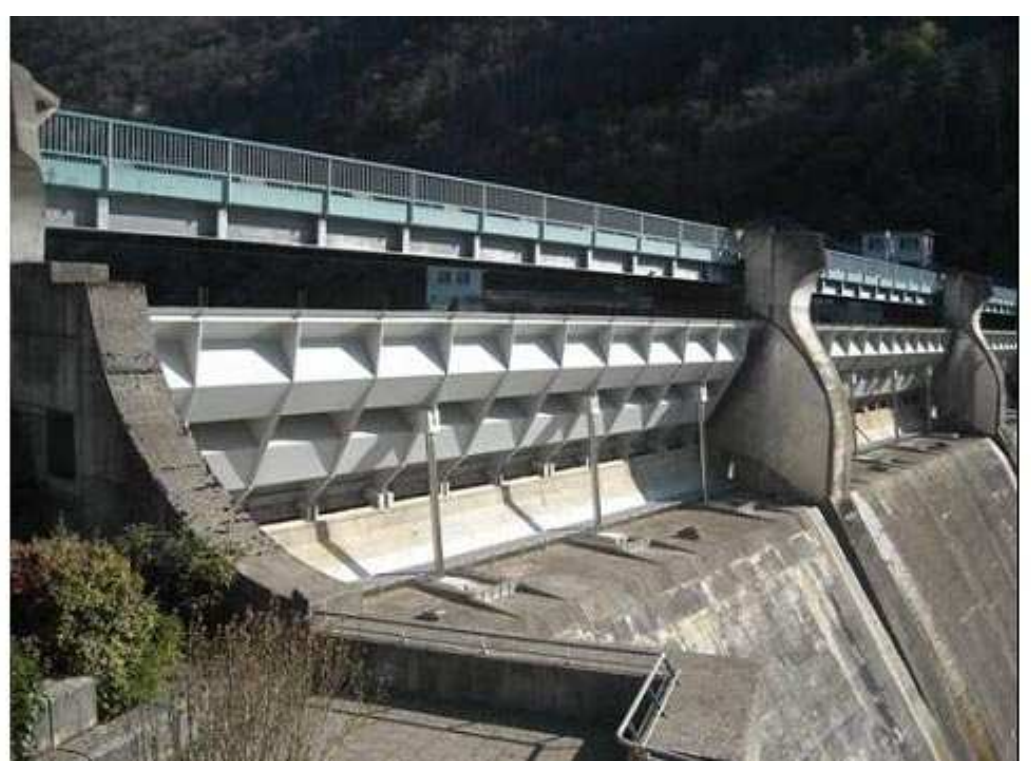

Photo 3.2 - Clapet, barrage de Pinet sur le Tarn 
Leur usage est réservé à un écoulement de surface et ils ne peuvent donc pas être utilisés comme vannes noyées. Le clapet est actuellement la vanne la plus utilisée pour les ouvrages neufs de navigation en rivière.

Les clapets peuvent avoir de très grandes largeurs (supérieures à 30 mètres), mais leur usage en rivière est aujourd'hui limité à des hauteurs de l'ordre de $6,50 \mathrm{~m}$ lorsqu'ils sont en régime partiellement noyé par l'aval, pour des raisons de vibrations engendrées par les fluctuations de pression directement en aval du clapet. L'utilisation de clapets nécessite la mise en place de becs de fractionnement permettant l'aération de la lame déversante pour de faibles ouvertures ainsi qu'un dispositif d'aération latérale réalisée par l'installation de tuyauteries dans les bajoyers afin de réduire les problèmes de vibrations.

Les clapets peuvent être manœuvrés par des vérins, des treuils à chaînes, des systèmes à crémaillère ou des dispositifs à flotteur ou bien encore par la charge d'eau de la retenue, via un contrepoids. Ils ne nécessitent généralement pas de superstructure et permettent le réglage raisonnablement précis des débits et la navigation en période de crues. Ils sont intéressants pour le passage des corps flottants.

Cependant, à cause de leur fonctionnement en vannes abaissantes, des précautions doivent être prises afin d'éviter la chute intempestive du clapet et la perte du plan d'eau en cas d'incident sur le système de manœuvre.

\subsection{Vannes secteurs ou vannes tambours}

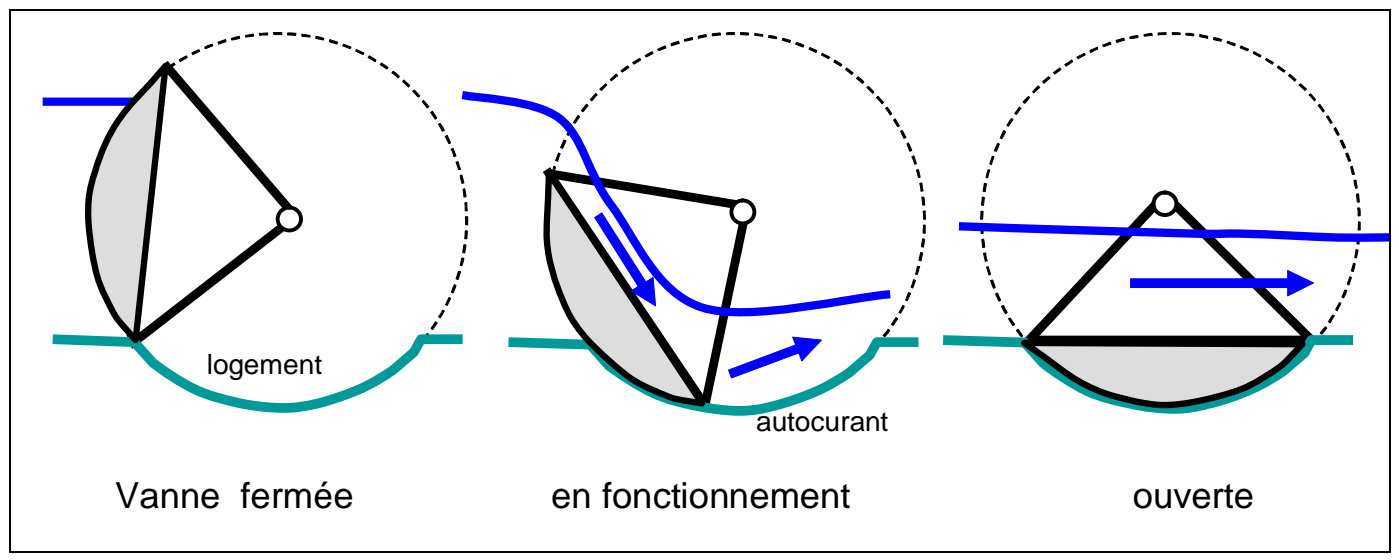

Figure 3.10 - Coupe type d'une vanne secteur

II s'agit d'une vanne avec déversement par dessus puis sur le bordé intérieur. La vanne s'efface complètement dans un logement aval prévu à cet effet. Ce logement est autocurant. 


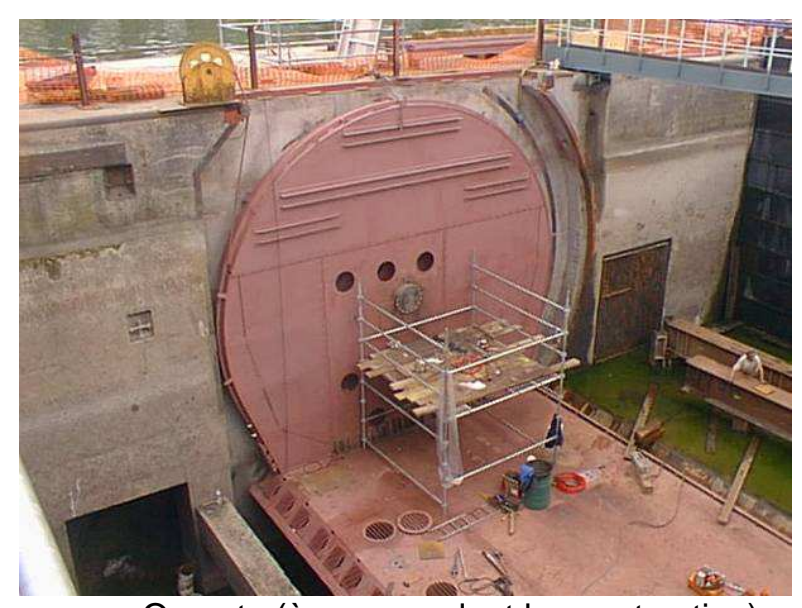

Ouverte (à sec, pendant la construction)

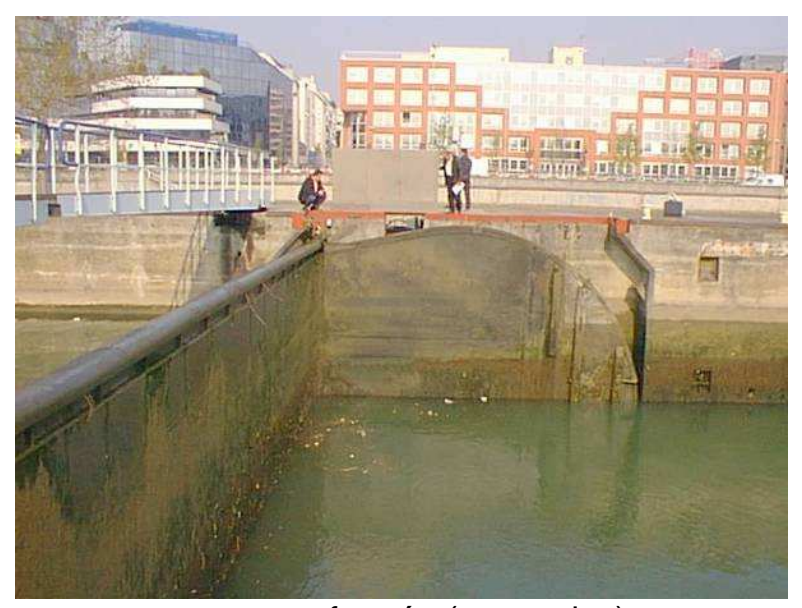

fermée (en service)

Photos 3.3 - Vanne tambour, écluse Andrésy

Le barrage du Couesnon, en baie du mont Saint Michel, est équipé de ce type de vanne, qui reste cependant rare.

\subsubsection{Vannes segments}

Les vannes segments sont des vannes levantes constituées d'un tablier de forme cylindrique assurant la bouchure du pertuis, prolongé par deux bras latéraux permettant le transfert des charges sur le béton des bajoyers par l'intermédiaire de tourillons et éventuellement de corbeaux (cf. fig. 3.11).

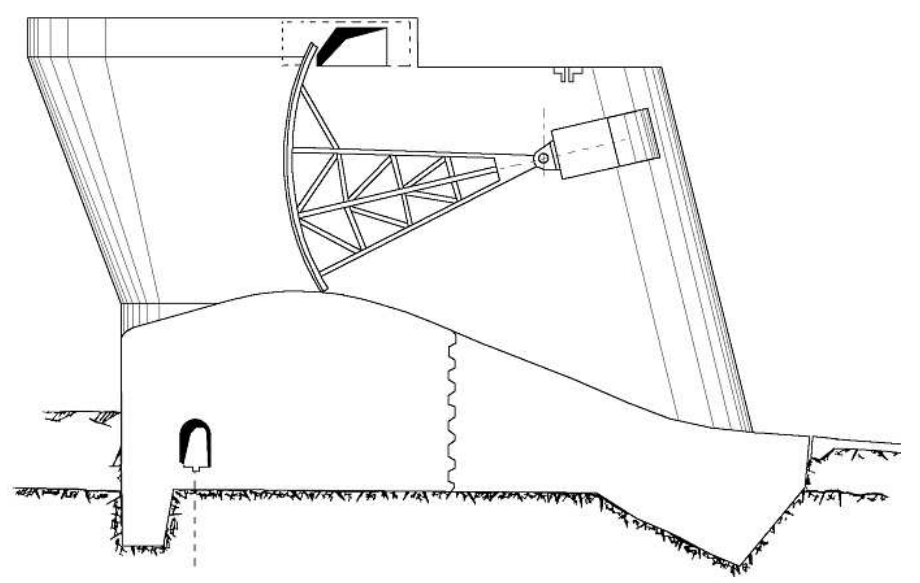

Figure 3.11 - Coupe type d'une vanne segment

Elles ont l'avantage de reporter la poussée de l'eau sur l'axe, les joints latéraux n'étant alors pas sollicités.

Ces vannes peuvent être installées soit en surface soit dans des pertuis de fond noyés.

Lorsqu'elles sont installées en surface, ces vannes peuvent être équipées d'un volet déversant permettant le passage des faibles débits et des corps flottants (cf. photo 3.4). Elles peuvent atteindre de grandes dimensions. 


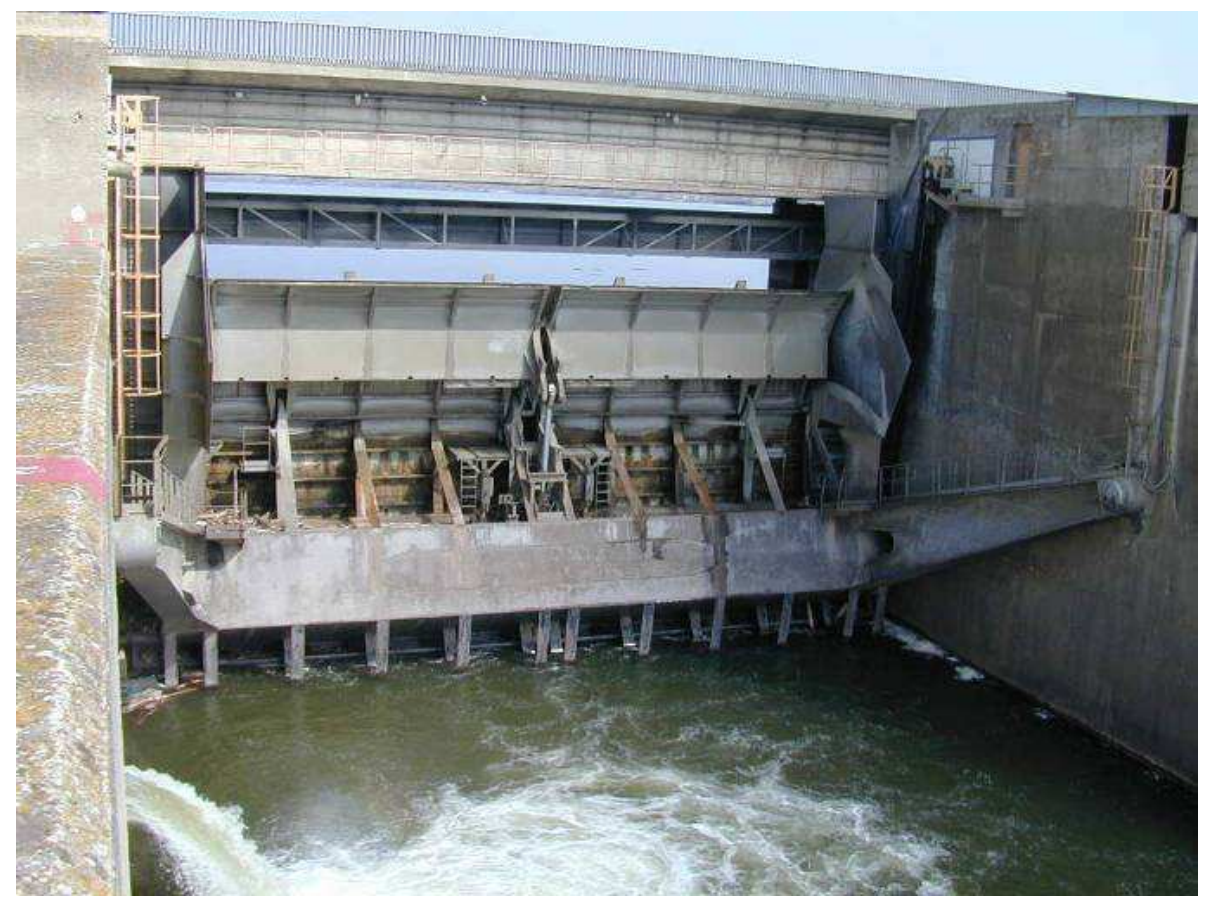

Photo 3.4 - Vanne segment munie d'un volet de surface, barrage de Caderousse

Lorsqu'elles sont utilisées en pertuis de fond, les dimensions sont généralement plus limitées du fait des difficultés d'intégration dans le corps du barrage et du fait des problèmes de déformation sous la pression de la retenue.

Ces vannes peuvent être manœuvrées par des vérins, des treuils à chaînes ou à câbles ou des systèmes à flotteur et contrepoids, et ne nécessitent pas de superstructure.

\subsubsection{Vannes wagons}

Les vannes wagons simples sont des vannes levantes constituées d'un tablier métallique plat, réalisé en un ou plusieurs éléments solidaires entre eux. Chaque élément possède des galets de roulement prenant appui sur des rails situés dans des rainures latérales réalisées dans le béton des bajoyers (cf. fig. 3.12).
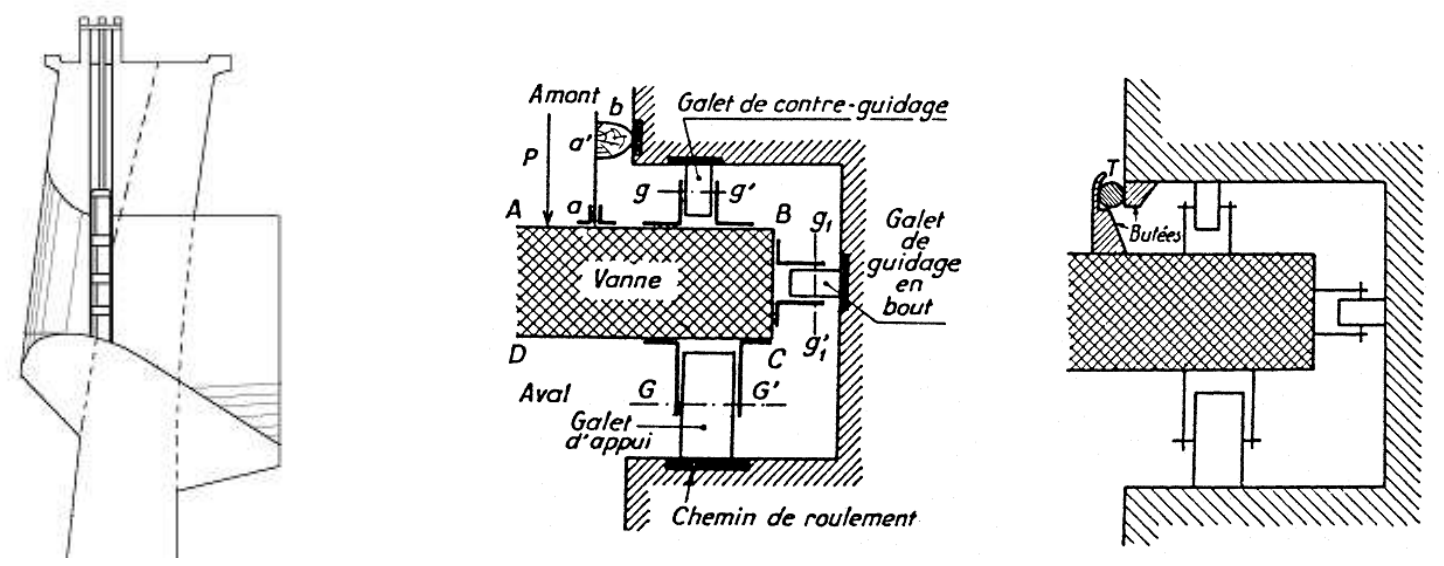

Figure 3.12 - Coupes types d'une vanne wagon 
Ces vannes peuvent être de grandes dimensions. Utilisées en rivière, elles peuvent également supporter un volet déversant pour le passage des petits débits et des embâcles. En vannes de fond, leur usage est limité par la présence des rainures latérales qui peuvent perturber sensiblement l'écoulement et générer des problèmes de cavitation.

Ces vannes sont simples et robustes, utilisent peu de place et sont faciles d'entretien. Elles sont très polyvalentes et peuvent être utilisées dans quasiment toutes les applications, en surface ou noyées, en conduite (avec carénage ou manœuvrées par brimbales), etc. Cependant, elles sont plus lourdes que d'autres types de bouchures et nécessitent une superstructure verticale importante. Comme les vannes segments, elles peuvent être manœuvrées à l'aide de vérins, de treuils à chaînes ou à câbles ou de vis sans fin. Cependant, elles ne sont pratiquement plus choisies sur les évacuateurs de crues des barrages neufs, du fait des efforts de manœuvre importants et de la nécessité de rainures latérales.

Les vannes wagons double corps (appelées aussi vannes crochets) sont exclusivement utilisées en rivières, où elles autorisent la navigation en période de crues tout en garantissant une grande souplesse de contrôle des débits et en permettant l'aération de la lame d'eau. Elles sont constituées d'un élément inférieur de type vanne wagon et d'un élément supérieur prenant appui sur le bordé amont de l'élément inférieur.

Ces deux systèmes permettent une très bonne régulation du plan d'eau amont: lorsque le débit augmente, on passe progressivement de l'écoulement de surface (bon régulateur de niveau amont mais de faible débitance) à celui de fond (écoulement en charge bénéficiant de toute la chute, donc de bonne débitance), puis l'ensemble est dégagé par le haut pour laisser le passage libre aux plus fortes crues. Ce type de vannes correspond à une utilisation astucieuse des lois de l'hydraulique et de la régulation. Cependant, ces vannes nécessitent des superstructures importantes, sont techniquement compliquées et leur entretien n'est pas aisé. En pratique, elles ont souvent été remplacées sur les aménagements plus récents par des vannes segments munies d'un clapet en surface (cf. photo 3.4).

\subsubsection{Autres types de vannes}

Les vannes citées précédemment constituent la grande majorité des vannes d'évacuateurs de crues, au moins en ce qui concerne les ouvrages neufs. On rencontre cependant d'autres types de vannes, plus ou moins répandus, sur les ouvrages en service.

\subsection{Vannes Stoney}

Proches dans leur conception des vannes wagons, elles s'en distinguent par l'existence de trains de galets indépendants roulant entre le tablier et le siège. Elles sont dotées de deux trains de galets (un fixe sur le siège et un mobile lié au tablier). Les trains de galets sont en général entraînés par une poulie qui chevauche un câble acier dont un brin est fixe et l'autre amarré au tablier. Le train de galets se déplace deux fois moins vite que le tablier de la vanne. Lorsque la vanne a parcouru sa hauteur, elle n'est plus en contact qu'avec la moitié du train de galet (cf. fig. 3.13). 

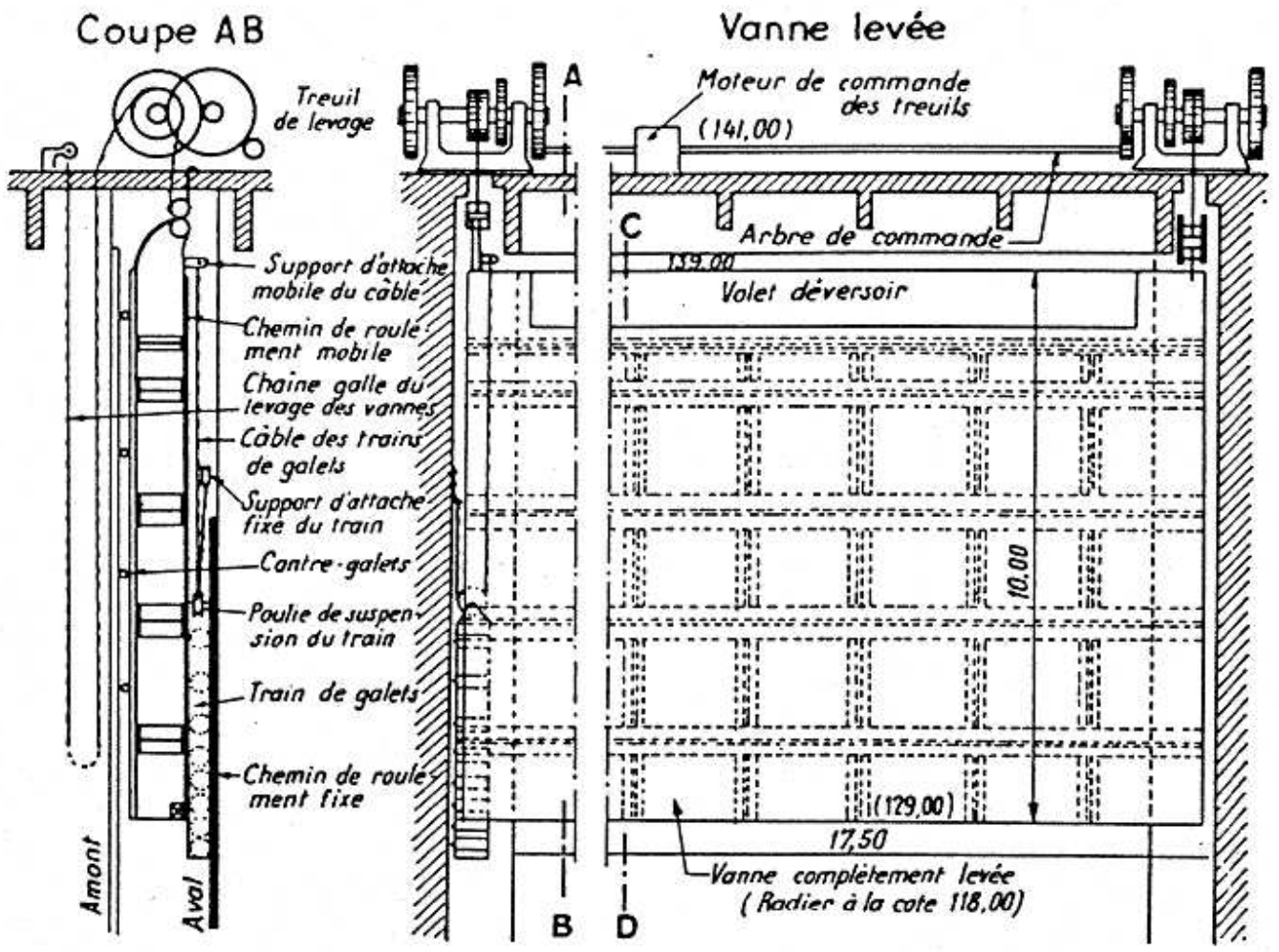

Figure 3.13-Coupe type d'une vanne Stoney

Ces vannes sont utilisées uniquement comme vannes de surface, elles présentent l'avantage d'une meilleure répartition des efforts sur les pièces fixes, ce qui est appréciable sur des maçonneries anciennes. Elles nécessitent des superstructures analogues à celles des vannes wagons. Le risque de coincement du train de galets (par des corps flottants ou autres) constitue leur point faible principal.

\subsection{Vannes papillons}

Ces vannes sont constituées d'un disque pivotant sur un axe dans la conduite qu'elles obturent, le disque étant manœuvré de l'extérieur par l'intermédiaire d'un bras. Elles sont classiquement utilisées en vidange de fond, mais quelques ouvrages en disposent en évacuateurs de crues. Le risque d'obturation par un bois ou un bloc rocheux constitue leur point faible principal (le papillon constitue un obstacle dans le conduit). De ce fait, ces organes n'apportent pas la meilleure sécurité de fonctionnement et garantie de débitance. II convient donc, dans le cas d'une conception nouvelle, d'appréhender avec prudence le choix de ce type de vanne.

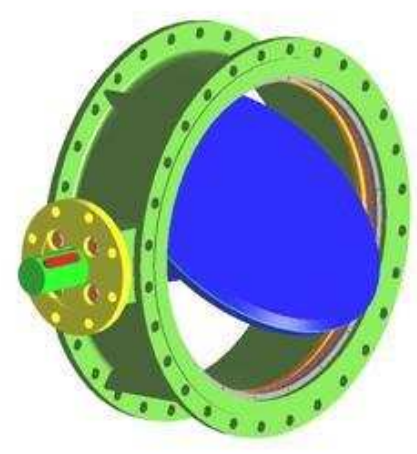

Figure 3.14 - Vanne papillon 


\subsection{Vannes à jet creux}

Ces vannes sont constituées d'une buse fuselée coulissant dans la conduite qu'elles obturent (deux variantes possibles: buse mobile et conduit fixe ou buse fixe et conduit mobile). Le jet passe autour de cette buse, d'où l'appellation de jet creux. Ces vannes sont classiquement utilisées en vidange de fond, mais quelques ouvrages en disposent en évacuateurs de crues. Comme pour les vannes papillons, le risque d'obturation par un bois ou un bloc rocheux constitue leur point faible principal (le pointeau constitue un obstacle dans le conduit). De ce fait, à l'instar des vannes papillons, ces organes n'apportent pas la meilleure sécurité de fonctionnement et garantie de débitance. II convient donc, dans le cas d'une conception nouvelle, d'appréhender avec prudence le choix de ce type de vanne. Ces vannes sont de très bons dissipateurs d'énergie.

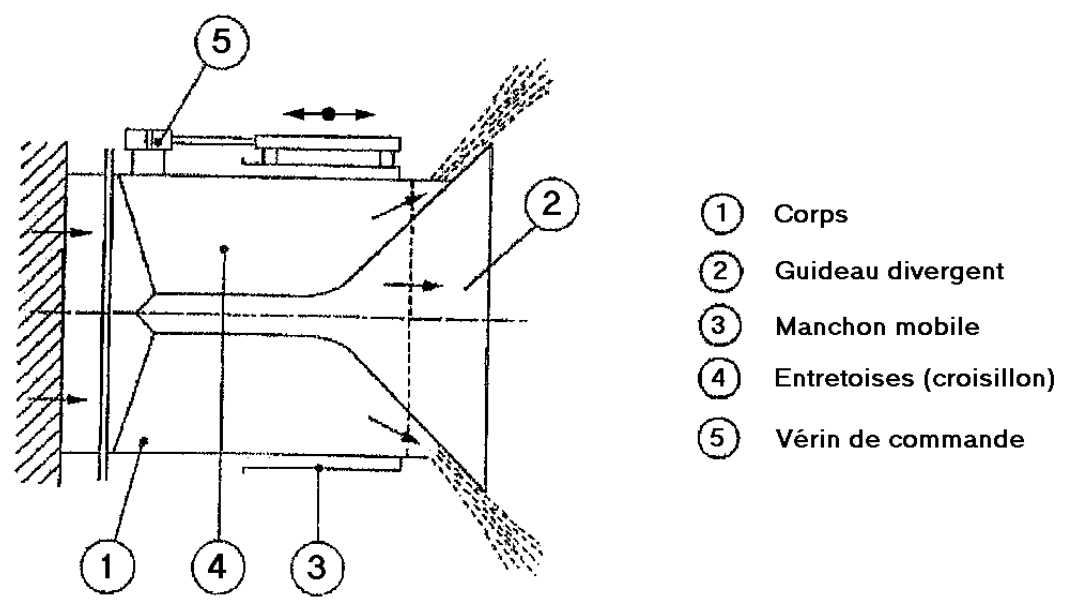

Figure 3.15 - Vanne à jet creux

\subsection{Autres types de vannes}

On pourrait également citer, de manière non exhaustive, l'existence d'autres types de bouchure : toits, aiguilles, hausses Aubert, pointeaux, guillotines, etc.

\subsubsection{Organes de manœuvre}

Les deux principaux types d'organes de manœuvre de vannes rencontrés sur les vannes de barrage sont les vérins hydrauliques et les treuils à chaînes. On peut également citer les treuils à câbles couramment utilisés dans les pays Anglo-Saxons et de l'Est, les vérins électromécaniques plus récemment utilisés, les systèmes vis-écrou, les crémaillères et enfin les dispositifs à flotteurs.

\subsubsection{Vérins hydrauliques}

Ce type de mécanisme a souvent la préférence actuelle du concepteur du fait de sa simplicité, sa relative facilité de mise en œuvre et de maintenance et son coût de fourniture plus faible que pour les autres types d'organes. Il est également possible d'intégrer les vérins dans les structures de façon très discrète. Associés à une centrale hydraulique, les vérins permettent une manœuvre souple et contrôlée des clapets et des vannes.

Ils présentent cependant l'inconvénient de fonctionner sous pression et sont susceptibles de fuites internes et externes qui peuvent être préjudiciables au réglage de la position des 
organes manœuvrés et engendrer de la pollution en cas de fuite (le rejet dans la nature d'huile est proscrit par le code de l'environnement, que l'huile soit biodégradable ou non). Ils nécessitent de nombreux composants pour leur fonctionnement, peuvent s'avérer être des éléments sensibles au séisme et, dans le cas de vérins doubles, la question de leur synchronisation est toujours un point de fragilité. Leur conception doit permettre le dépannage en situation d'urgence.

Du point de vue du dimensionnement, ces systèmes sont limités par la course des vérins.

\subsubsection{Treuils à chaîne}

Les treuils à chaîne utilisés pour la manœuvre des organes de bouchure (vannes, clapets...) sont constitués d'un réducteur et d'une chaîne de type "Galle ", elle-même constituée de tôles d'acier formant les maillons et d'axes appelés fuseaux. Les chaînes peuvent posséder plusieurs mailles (doubles, triples ou quadruples) en fonction de l'effort de levage.

Les treuils à chaîne sont robustes et éprouvés, et leur durée de vie peut être extrêmement longue avec une maintenance souvent relativement simple, surtout en ce qui concerne les réducteurs, qui peuvent fonctionner très longtemps avec un entretien mineur.

Les chaînes de liaison avec l'organe manœuvré doivent faire l'objet d'une maintenance et d'une surveillance adaptées. Les très hautes pressions diamétrales des axes sur les maillons engendrent des difficultés de graissage et le grippage de ce type de chaîne est possible. II est nécessaire de prévoir une maintenance régulière (périodicité variable en fonction de l'exposition des chaînes aux intempéries et aux déversements de la vanne), qui consiste en un graissage soigné, un contrôle d'allongement du "pas » de la chaîne et en une manœuvre aussi complète que possible de la chaîne pour conserver sa "souplesse ". Pour les tronçons les plus exposés, l'utilisation de chaînes en acier inoxydable, éventuellement montées sur antifriction, est envisageable mais cette solution est relativement coûteuse.

Le coût de fourniture et d'installation de moto-réducteurs est, par ailleurs, plus élevé que celui du vérin.

\subsubsection{Treuils à câble}

Les treuils à câbles sont également utilisés sur les vannes d'évacuateur de crues. Ils présentent des avantages comparables à ceux à chaînes (simplicité de conception, robustesse). La possibilité de mouflage permet de réduire la puissance des treuils au prix d'une certaine complexité. La maintenance des câbles doit être soignée (graissage et contrôles). Les règles de dimensionnement des treuils peuvent s'inspirer des règles édictées par la Fédération Européenne de Manutention (FEM, 1998). Les contrôles des câbles peuvent être réalisés en appliquant les critères proposés par la norme ISO 4903 «Appareils de levage à charge suspendue - Câbles - Entretien, maintenance, installation, examen et dépose ».

\subsubsection{Crémaillères}

De conception simple et robuste, ce dispositif peut généralement être équipé d'une commande de secours manuelle (manivelle). La maintenance est simple. D'un point de vue du dimensionnement, ce type de dispositif est limité par la longueur de la crémaillère. 


\subsubsection{Systèmes de contrôle-commande}

Le système de contrôle-commande, ou système de surveillance et de conduite, doit permettre :

- d'assurer la commande des ouvrages (ouverture et fermeture des vannes) par les ordres manuels d'un opérateur, ou automatiquement en fonction de règles prédéfinies basées sur des mesures de niveaux ou de débit d'eau, mesures situées au niveau de l'ouvrage, en amont et/ou en aval afin d'anticiper sur l'évolution de la retenue d'eau ou du bief amont ;

- de surveiller le bon état de l'ensemble de l'installation et de reporter en temps réel cet état ainsi que tous les défauts détectés. Le système doit pouvoir contrôler lui-même la validité des indications données par les capteurs, le bon fonctionnement des appareillages et les ordres aberrants.

Le système doit être conçu en fonction des caractéristiques et objectifs de l'ouvrage, des modes de gestion envisagés et du niveau de sécurité requis: conception globale de l'ouvrage, modes de gestion envisagés dans les différents cas de fonctionnement et notamment lors du passage des crues (manuel ou automatique, localement ou à distance), niveau de sécurité requis, importance du risque en cas de défaillance.

L'architecture des systèmes de contrôle-commande comprend généralement :

- les interfaces homme-machine (écran de visualisation et de commande, pupitre, pupitre de marche dégradée) ;

- les capteurs (niveaux, positions, ...) ;

- les automatismes de surveillance et de conduite (régulation) ;

- les automatismes de sauvegarde (sécurité) ;

- les armoires de commande de puissance des actionneurs et/ou des auxiliaires (groupes hydrauliques...) ;

- le système de communication à distance pour le report des états et des défauts. Les liaisons couramment utilisées sont: réseau numérique privé, ligne téléphonique commutée, liaison hertzienne, GSM, réseau internet...

Les dispositions relatives à la gestion des crues sont parmi les plus importantes en ce qui concerne la sécurité des ouvrages.

L'analyse de ces contraintes et objectifs permet de décider du niveau de redondance des capteurs, des automates de régulation et des actionneurs, et des modes de commande, en marche normale mais aussi en marche dégradée, avec l'ensemble des cas de fonctionnement partiel des installations (équipements indisponibles, en défaut ou en maintenance).

Une attention particulière doit être apportée à la fiabilité des équipements de contrôlecommande, à leur robustesse à supporter les crues, à leur nombre suffisant pour s'assurer d'une acquisition fiable des données, à leur implantation à l'abri des embâcles, des apports de sédiments et des dégradations volontaires. II en est de même pour ce qui concerne leur entretien (étalonnage, tests, ...).

Par ailleurs, un dispositif de sauvegarde doit être prévu. Ce dispositif, indépendant des autres matériels, permet d'assurer l'alerte de l'exploitant, voire l'arrêt de la conduite automatique et les manœuvres de mise en sécurité de l'ouvrage, lorsqu'on s'écarte des conditions d'exploitation normale (cf. 3.3.4, en particulier 3.3.4.2). 


\subsubsection{Commandes hydrauliques à partir de la retenue}

On trouve sur les aménagements hydrauliques existants un nombre important de ce type de vannes ou clapets, motorisés sans apport d'énergie externe, par une commande à flotteurs et contrepoids. Ces vannes ou clapets sont souvent installés sur des évacuateurs de crues.

Les conceptions de ces dispositifs sont multiples, depuis le clapet à contrepoids " simple » s'ouvrant sous la pression hydrostatique et fonctionnant en tout ou rien, jusqu'à des clapets ou des vannes de type secteur disposant de chambres de réglage dont le débit d'alimentation et d'évacuation est réglable et qui permettent un fonctionnement en régulation de niveau d'un plan d'eau.

Pour bien exploiter de tels organes, il est primordial de bien comprendre le fonctionnement de leur commande et de bien appréhender ses limites. Si elles sont maintenues correctement, les vannes à flotteurs constituent des organes de sécurité fiables du fait de leur ouverture automatique sans énergie.

Ces organes tirent leur énergie de manœuvre de la retenue et sont commandés automatiquement par influence directe du niveau de la retenue, soit sur le tablier lui-même, soit sur le remplissage de chambres à flotteurs, avec ou sans l'aide de contrepoids.

Parmi les avantages de ce système, il faut citer :

- le fractionnement des risques de défaut (si la commande est individuelle) ;

- une manœuvre indépendante de toute source d'énergie électrique ;

- une manœuvre indépendante de tout automatisme électrique.

Mais ces systèmes présentent aussi un certain nombre de risques. Ces risques ou inconvénients doivent être examinés dans le détail pour apprécier précisément la possibilité de doter le barrage de ce type d'organe, parmi lesquels :

- les risques d'ouvertures intempestives ;

- les risques de non ouverture : en cas de blocage, en particulier mécanique ou par défaut du circuit hydraulique (obstruction, gel du flotteur), il n'existe généralement pas de manœuvre de secours. En particulier, les alimentations des chambres de manœuvre sont des points à surveiller (risque de bouchage par les débris flottants) ;

- les gradients de débit à la première ouverture peuvent être très forts. Ces vannes ne sont en général pas aptes à régler de manière précise un débit à restituer ;

- certains de ces systèmes présentent l'inconvénient d'un faible couple de manœuvre, qui les rend sensibles aux coincements.

\subsection{Conception des équipements}

\subsection{1 Équipements électromécaniques}

\subsubsection{1 Équipements mécaniques}

Les dispositions constructives à mettre en œuvre sont spécifiques aux ouvrages, aux types de vannes, à leur fonction et à leur importance, et devront être élaborées au cas par cas. On donne ici des recommandations communes relatives aux organes de manœuvre et aux dispositifs de maintien en position de sécurité. D'une manière générale, il est courant de 
prévoir plusieurs redondances (sur l'alimentation électrique, sur les moyens de manœuvre des organes, etc.).

\subsection{Organes de manœuvre}

Les organes de manœuvre doivent être dimensionnés pour les efforts calculés dans la conception des vannes. On doit tenir compte des efforts et charges «parasites », liés par exemple aux déformations des équipements et aux efforts de manœuvre, en tenant compte du fait que ces efforts sont difficiles à appréhender.

La vitesse de manœuvre doit également être prise en compte dans la conception du système. La vitesse d'ouverture des vannes et le débit associé doivent en effet être capables de suivre le gradient de montée de la crue, sous peine de voir la cote de consigne dépassée voire le barrage submergé. La détermination du gradient maximum de montée de crue est un donc un paramètre primordial à déterminer par l'étude hydrologique.

La décrue étant généralement plus lente, la vitesse de fermeture des vannes pourra suivre le gradient maximum de décrue. II est à noter qu'une vidange rapide de la retenue peut générer des glissements de terrain.

Les vannes possèdent chacune leur propre organe de manœuvre et doivent pouvoir être manœuvrées sous toutes les conditions de charge et être en mesure de tenir une position quelconque entre la fermeture et l'ouverture complète.

Les vannes et leurs organes de manœuvre doivent être équipés de dispositions de sécurité, adaptées aux organes à manœuvrer et définies en fonction d'une analyse de risques. Sauf justifications spécifiques, au minimum les dispositions suivantes devront être prises en compte :

- pour les organes de manœuvre, ces dispositions doivent inclure des arrêts en fin de course, fonctionnement par paliers, système de reprise de fuite sur les vérins, limitation de la vitesse d'abaissement des clapets en cas d'incident ;

- pour l'énergie de manœuvre, ces dispositions doivent inclure un dispositif d'alimentation de secours en énergie, tels qu'un groupe de secours à moteur thermique, ou éventuellement un dispositif faisant appel à la force manuelle.

Pour les vannes participant à l'évacuation des crues, la position fermée n'étant pas nécessairement la position la plus sûre pour cette fonction, on ne recommande pas de les munir de la disposition systématique de fermeture sous leur propre poids sans apport d'énergie.

\subsection{Maintien en position des éléments mécaniques}

Le maintien en position des éléments mécaniques doit être garanti en cas de défaillance de l'organe de manœuvre. Les solutions sont multiples et peuvent être constituées d'un crochet venant agripper la porte, d'un verrou (le déplacement d'un axe dans une oreille de levage vient en retenir la structure) ou encore de poutres de stockage que l'on glisse sous la porte en position haute, l'organe de manœuvre étant ensuite libéré en posant la porte sur ces poutres.

Il convient de faire la distinction entre deux types de verrous :

- d'une part les verrous « de sécurité », qui ont pour fonction la condamnation de l'organe lorsqu'il est nécessaire de travailler à l'aval ; 
- d'autre part les verrous « d'exploitation », destinés à éviter, en exploitation normale, un abaissement intempestif du clapet.

L'adjonction d'un verrou "d'exploitation », venant mettre un obstacle au fonctionnement « naturel » de l'installation, est considérée comme un pis-aller, cette technique doit généralement être évitée. Lorsque la fonction de l'organe est d'assurer la tenue de la cote à une valeur donnée (généralement à $\mathrm{RN}$ ), le verrouillage des clapets est contre-indiqué.

\subsubsection{Alimentation électrique}

\subsection{Principes généraux}

L'expérience d'épisodes de crues importants montre que les lignes du réseau d'alimentation de distribution électrique ne sont pas à l'abri d'incidents de fonctionnement (tempête, foudre, chute d'arbres...).

Le Guide pour le contrôle des barrages en exploitation (BETCGB, 2002) recommande pour assurer l'alimentation électrique des évacuateurs de crues vannés, notamment en cas de conditions météorologiques extrêmes, de prévoir :

- une alimentation principale (dite «normale »), fournie par le réseau de distribution, éventuellement doublée par les auxiliaires de l'usine pour les ouvrages hydroélectriques;

- une alimentation de secours à partir d'un groupe électrogène totalement autonome et dédié au barrage.

Ces différents niveaux d'alimentation électrique ne doivent pas être affectés d'un défaut commun. En particulier, les chemins de câblage de l'alimentation principale et des alimentations de secours doivent être autant que possible différenciés afin qu'un défaut n'affecte pas simultanément les deux sources d'alimentation.

La puissance installée doit permettre de manœuvrer les organes mécaniques suffisamment rapidement par rapport aux vitesses maximales d'augmentation des débits à évacuer sous l'effet de la crue de projet.

\subsection{Conception des circuits électriques}

La distribution de l'énergie alternative doit être traitée avec soin.

Il faut en particulier éviter tous les points communs entre la source normale et celle de secours qui, en cas d'incendie par exemple, risqueraient de priver l'évacuateur de son énergie de manœuvre. Les cheminements des câbles d'alimentation doivent être indépendants (entre la voie normale et la voie de secours). Dans le cas où le croisement ou le cheminement commun (forage) ne peut être évité, il convient de prendre toutes les dispositions pour isoler les deux liaisons l'une par rapport à l'autre (plâtre, gaine ignifugée...).

Le matériel électrique de manœuvre et de commande doit être installé de telle sorte qu'il soit hors d'eau et accessible pour un niveau de retenue a minima égal à celui des PHE. Un niveau de retenue plus élevé pourra être envisagé au vu d'une analyse des risques.

Les câbles doivent être protégés contre les risques d'écrasement, d'arrachement ou d'emportement de supports (en particulier sur les passerelles ou les ponts). 
Le schéma d'alimentation électrique des organes devra présenter des dispositions visant à :

- assurer des modes dégradés d'alimentation avant une coupure totale des circuits d'alimentation des vannes d'évacuation des crues ;

- générer des alarmes graduées en fonction du risque de défaillance d'alimentation du matériel concerné.

Ces principes sont assurés notamment par l'élimination maximale des modes communs.

\subsection{Alimentation de secours: groupe électrogène barrage}

Le groupe électrogène de l'alimentation de secours n'alimente que les organes de l'évacuateur de crues et les équipements essentiels à la sûreté d'exploitation du barrage : éclairage des passes et moyens d'accès, automatismes de surveillance et de conduite, ...

Le groupe électrogène du barrage est installé à demeure au barrage et à un emplacement :

- hors d'eau (au-dessus de la cote des plus hautes eaux envisageables) ;

- accessible pour un niveau d'eau a minima égal à celui des PHE ;

- isolé de la source d'alimentation principale.

Il doit être autonome (en source d'énergie de démarrage, tension de contrôle commande, fuel, refroidissement...).

L'autonomie du groupe de secours doit être suffisante pour assurer les manœuvres pendant la crue. Le fonctionnement réel du groupe électrogène doit être pris en compte, avec des marges de sécurité (prise en compte d'une situation perturbée). La capacité installée en carburant couramment admise est de 72 heures à pleine charge (à moduler en fonction du contexte : accessibilité du site, aménagement ou ville à proximité, moyens humains, etc.).

La puissance du groupe de secours est déterminée pour pouvoir assurer, outre l'alimentation de tous les équipements auxiliaires dédiés au barrage (éclairage, atelier d'énergie...), toutes les manœuvres et actions nécessaires:

- manœuvre des vannes, notamment le décollement ;

- transit du débit lorsque le gradient de débit entrant est maximal ;

- le cas échéant, compensation par les vannes du barrage du débit turbiné par l'usine hydroélectrique associée lors d'un déclenchement de cette usine.

Ainsi, il doit supporter l'appel de courant dû à la mise en mouvement de la dernière vanne nécessaire au passage du gradient maximal de débit, les autres vannes étant en régime établi de manœuvre.

\subsubsection{Protection des matériels électriques}

Le niveau de protection des matériels électriques est, dans les normes de fabrication françaises, caractérisé par deux indices :

- l'indice $\mathrm{IP}_{\mathrm{xy}}: \mathrm{x}$ est relatif à la protection contre les corps solides (variant de 0 à 6 ), incluant la protection contre les poussières pour les indices élevés (indice 6 : protection totale sauf pour machines tournantes (moteurs)), y est relatif à la protection contre les liquides (en l'occurrence, condensation-gouttes-projections de liquides-immersion, selon une progression de l'indice de 0 à 8 ) ; 
- l'indice $\mathrm{IK}_{\mathrm{z}}$ : protection mécanique contre les chocs ( $\mathrm{z}$ : indice (de 0 à 8 ) caractéristique d'une énergie de choc que l'appareillage doit absorber).

Les équipements concernés sont les armoires et les coffrets de commande, les automatismes, les motorisations électriques, le matériel électrique annexe et la connectique, les capteurs et les sondes intervenant dans la chaîne de surveillance et de commande des organes d'évacuation (liste non exhaustive).

Très souvent, les matériels choisis dans une gamme standard d'un fabricant ont des indices prédéterminés, en fonction surtout de leur installation, soit à l'intérieur d'un local fermé, soit à l'extérieur, donc soumis aux intempéries, voire au risque de submersion temporaire.

Les coffrets de protection électrique sont également utiles pour la protection contre les intrusions (rongeurs, etc.).

II faut faire attention à ne pas dégrader l'indice de protection d'un coffret ou d'une armoire par des montages ultérieurs non prévus : grilles de ventilation, câbles supplémentaires, etc.

\subsubsection{Ultimes secours}

Des possibilités d'intervention manuelle organe par organe doivent être prévues en ultime secours. En effet, la défaillance des dispositifs de commande et de motorisation principaux des vannes n'est pas à exclure.

Ainsi, au-delà du secours de l'alimentation électrique, le Guide pour le contrôle des barrages en exploitation (BETCGB, 2002) recommande également de prévoir un ultime secours permettant d'actionner directement l'organe de manœuvre (moteur thermique pour les vannes à treuils, pompe de secours alimentée par un mini-groupe électrogène pour les vannes à commande oléo-dynamique).

Le dispositif d'ultime secours est le moyen, utilisé en dernier recours, pour manœuvrer un organe d'évacuation de crue lorsque les moyens classiques sont inopérants : alimentation normale et secours, motorisation principale et son contrôle commande.

Ce moyen doit permettre de stabiliser une situation qui se dégraderait (et pourrait mener à l'incident) inexorablement sans celui-ci, en permettant de stopper l'exhaussement de niveau face à un gradient de débit fixé en manœuvrant un à un les organes. À ce titre, la vitesse de manœuvre d'un organe manœuvré par le dispositif d'ultime secours peut être inférieure à la vitesse normale tant que l'objectif de stabilisation de la situation est garanti.

Le dispositif d'ultime secours concerne a minima l'ouverture des organes de sécurité mais peut également être prévu pour assurer leur fermeture.

Cet ultime secours n'a de sens qu'une fois l'exploitant sur place, ce qui signifie que le paramètre « temps d'accès » n'est pas à considérer dans son dimensionnement. Par contre, le délai et les moyens humains nécessaires à sa mise en œuvre doivent être compatibles avec l'organisation de l'exploitation pour tenir l'objectif. Cette compatibilité doit prendre en compte le séquençage selon lequel sont manœuvrés les organes et le délai de mise en œuvre du dispositif d'ultime secours, notamment dans le cas de dispositifs mobiles et communs à plusieurs organes. 
Parmi les solutions d'ultime secours, on peut envisager :

- vannes à commande par treuils : un moteur thermique ou un groupe électrogène sur un chariot avec moteur électrique de secours à demeure ou démontable peut être embrayé sur les réducteurs mécaniques ;

- vannes à commande oléodynamique: une pompe de secours fixe ou un groupe électrogène avec pompe monté sur un chariot peut être raccordé par flexible sur le circuit hydraulique. En cas de doubles vérins (un sur chaque rive), la synchronisation entre rives doit être assurée. Si les dispositions de sécurité ultime incluent le fonctionnement en mode dégradé avec un seul vérin, il faudra vérifier que le corps de la vanne est suffisamment rigide pour reprendre les efforts et ne pas entraîner de coincement.

Dans tous les cas, l'alimentation en énergie et le contrôle commande de l'ultime secours doivent être indépendants des auxiliaires du barrage (normaux et secourus) et de l'automatisme de $1^{\mathrm{er}}$ rang de l'organe.

Pour des barrages équipés de vannes de faible dimension, un ultime secours manuel (manivelle, pompe à main) peut être admissible, s'il satisfait aux exigences de temps de manœuvre, c'est-à-dire s'il est compatible avec le gradient maximal de débit retenu, en prenant en compte la disponibilité du personnel d'exploitation ainsi que son endurance. Généralement, l'utilisation des manivelles pour la manœuvre en ultime secours est à proscrire sur les grandes vannes, car les vitesses de manœuvre sont très lentes et conduisent à l'épuisement rapide des exploitants.

En outre, afin de gérer la conduite de l'ouvrage en situation dégradée, il est nécessaire que l'exploitant dispose des moyens suivants :

- position des organes : une règle mécanique positionnée au droit de chaque organe. Elle doit être a minima visible depuis le point de commande de l'ultime secours ;

- cote du plan d'eau : une échelle limnimétrique implantée dans la retenue (cf. 3.3.3.1.1) ;

- éclairage adapté à la mise en œuvre du dispositif d'ultime secours (variable suivant la solution technique de l'ultime secours). Rappelons que la situation dégradée pour laquelle la mise en œuvre de l'ultime secours est nécessaire peut provenir de la perte de toute source d'énergie du barrage (normale et secours).

\subsubsection{Commande des vannes}

\subsubsection{Automatisation des vannes}

La conduite d'un évacuateur de crues doit toujours pouvoir se faire en manuel, mais il est possible d'envisager une automatisation des actions dans les conditions suivantes :

- dans le cas de la gestion de l'ouvrage automatisée à l'aide d'un système de contrôle commande, le fonctionnement en mode dégradé, voire la reprise de la conduite en mode manuel, doivent impérativement être pris en compte ;

- même si la conduite automatisée des organes mécaniques ne nécessite pas nécessairement une présence humaine (du point de vue de contraintes techniques), les consignes d'exploitation doivent prévoir une présence humaine au-delà d'un certain niveau de crue ; des dispositions techniques sont alors intégrées pour ne pas dépasser ce niveau (limitations des fonctions automatisées) et pour assister l'opérateur en crue (par exemple, validation des ordres proposés par l'automate).

Par ailleurs, toutes les vannes et tous les systèmes de manœuvre d'un évacuateur de crues ne se prêtent pas à une marche automatique dans des conditions satisfaisantes. Avant de 
procéder à une telle automatisation, il faut se poser la question de l'adéquation du matériel existant à un fonctionnement automatique, avec l'esprit critique suffisant.

\subsubsection{Commandes nécessitant une source d'énergie externe}

\subsection{Points ou niveaux de commande}

II n'est pas recommandé de commander l'ouverture à distance des vannes de barrage (évacuateurs de crues, vidanges de fond...) à partir d'une télécommande située hors du site du barrage: au-delà du risque d'ouverture intempestive, la commande à distance de l'ouverture des vannes de barrage introduit un retard dans les interventions consécutives à la défaillance du dispositif. De plus, le recours à cette commande se situe en général au moment où la probabilité de défaillance des circuits de transmission est la plus grande. En effet, les conditions climatiques sont un facteur commun entre les incidents sur le parcours des liaisons de télétransmissions et le besoin d'utilisation des vannes de contrôle des crues.

\section{II est recommandé de prévoir la commande des vannes à partir de deux, voire trois points (ou niveaux) de commande :}

- au niveau de chacune des vannes avec une commande manuelle ;

- depuis un tableau général pour la conduite du barrage (mode distance/manuel) ;

- et le cas échéant à partir de l'automate de conduite (mode distance automatique).

Les liaisons entre les points de commande, les vannes et les capteurs nécessaires à la gestion doivent éviter les sources de défaut commun.

\section{Commande manuelle locale (au niveau de chacune des vannes)}

Il est recommandé de prévoir d'une part des commandes manuelles éventuellement complétées par des commandes automatiques situées dans le local commande, et d'autre part des commandes manuelles situées au niveau des organes de manœuvre et des centrales hydrauliques. En outre, la vanne doit a minima pouvoir être commandée en manuel local depuis son armoire d'automatisme (mode local/manuel).

\section{Commande depuis le tableau général de conduite du barrage}

Une commande manuelle peut être mise en place depuis un poste de commande centralisée dans le local barrage (mode distance/manuel), ou en un autre lieu, par exemple salle de commande de l'usine si celle-ci est accolée au barrage, et si l'accès rapide aux évacuateurs depuis l'usine est garanti en toutes circonstances.

\section{Commande à partir de l'automate de conduite}

La vanne peut, le cas échéant, être commandée par un système de conduite automatique (mode distance/automatique) situé au barrage, ou dans un lieu proche (par exemple usine hydroélectrique, si celle-ci est accolée au barrage).

Dans le cas de la gestion de l'ouvrage à l'aide d'un automate de conduite, le fonctionnement en mode dégradé, voire la reprise de la conduite en mode manuel, doivent impérativement être pris en compte. L'automate doit, de plus, être en mesure de pouvoir contrôler lui-même la validité des indications données par les capteurs, le bon fonctionnement des appareillages et les ordres aberrants. 
La vanne peut, le cas échéant, être commandée à partir du dispositif de sauvegarde du barrage (dispositif de sécurité ultime du système de surveillance et de conduite, cf. 3.3.4.2).

\subsection{Contrôle-commande local ou de « $1^{\text {er }}$ rang »}

Chaque organe de crue est équipé d'un automatisme local (dit de « $1^{\text {er }}$ rang "), dédié et indépendant des autres organes de crue, assurant sa manœuvre et sa protection intrinsèque.

Lorsqu'une passe comprend deux organes d'évacuation (organe inférieur et organe supérieur), dont le fonctionnement est lié, on admet que l'automatisme de $1^{\text {er }}$ rang soit commun aux deux organes.

Les actions de commandes manuelles locales et le contrôle commande de la manœuvre ne doivent dépendre que des seules sources d'énergie nécessaires à la manœuvre des vannes. Le principe retenu est que les commandes manuelles restent opérantes, tant que l'on dispose, ou qu'il existe une possibilité d'alimenter en énergie électrique (voire sous une autre forme telle qu'hydraulique) les organes de manœuvre des vannes.

L'automatisme gère les protections mécaniques ou hydrauliques qui visent à empêcher le blocage de l'organe ou la dégradation de sa chaîne cinématique.

Une attention toute particulière sera portée aux protections permettant de détecter un blocage de l'organe de crue en phase d'ouverture (surcharge) ou un blocage de l'organe de crue en phase de fermeture (sous-charge ou mou de câble).

Dans le cas où des organes sont commandés par deux systèmes de motorisation situés chacun dans une des piles, non synchronisés mécaniquement, la synchronisation des deux systèmes est assurée par l'automatisme de $1^{\text {er }}$ rang et dans tous les cas par un dispositif indépendant des automates de conduite. Le choix de ce type de motorisation doit préalablement être évalué pour un fonctionnement en ultime secours (cf. 3.3.1.4), l'automatisme de $1^{\mathrm{er}}$ rang étant considéré hors service dans ce cas.

\subsection{Dispositif de crantage}

Un système de commande par cran (dit également «par palier » ou «par pas ") doit être privilégié dans le but d'assurer une manœuvre maîtrisée des organes (vannes ou clapets).

Ces crans sont issus d'un système à base de fins de course totalement indépendants des autres automatismes et de préférence liés le plus directement possible au mouvement de la vanne.

La valeur d'un cran élémentaire est liée à la valeur maximale de variation de débit admise à l'aval.

En cas d'envoi d'un ordre permanent (détecté par dépassement du cran), le système se verrouille, génère une alarme et n'exécute plus aucun ordre jusqu'à l'intervention manuelle de l'opérateur.

Indépendamment et en complément de ce dispositif de crantage, un contrôle du temps maximal de manœuvre continue de chaque vanne (par temporisation) est recommandé. Cette sécurité vérifie que les contacteurs de commande ne restent pas fermés au-delà du temps nécessaire pour réaliser un cran de manœuvre. 
En cas de défaut détecté par la temporisation de fonctionnement trop long, l'alimentation de puissance de la vanne ou de la passe doit être déclenchée et une alarme lancée.

La figure 3.16 ci-dessous explicite les notions d'arrêt sur cran, de protection par dépassement de cran et de protection par temps de fonctionnement trop long.

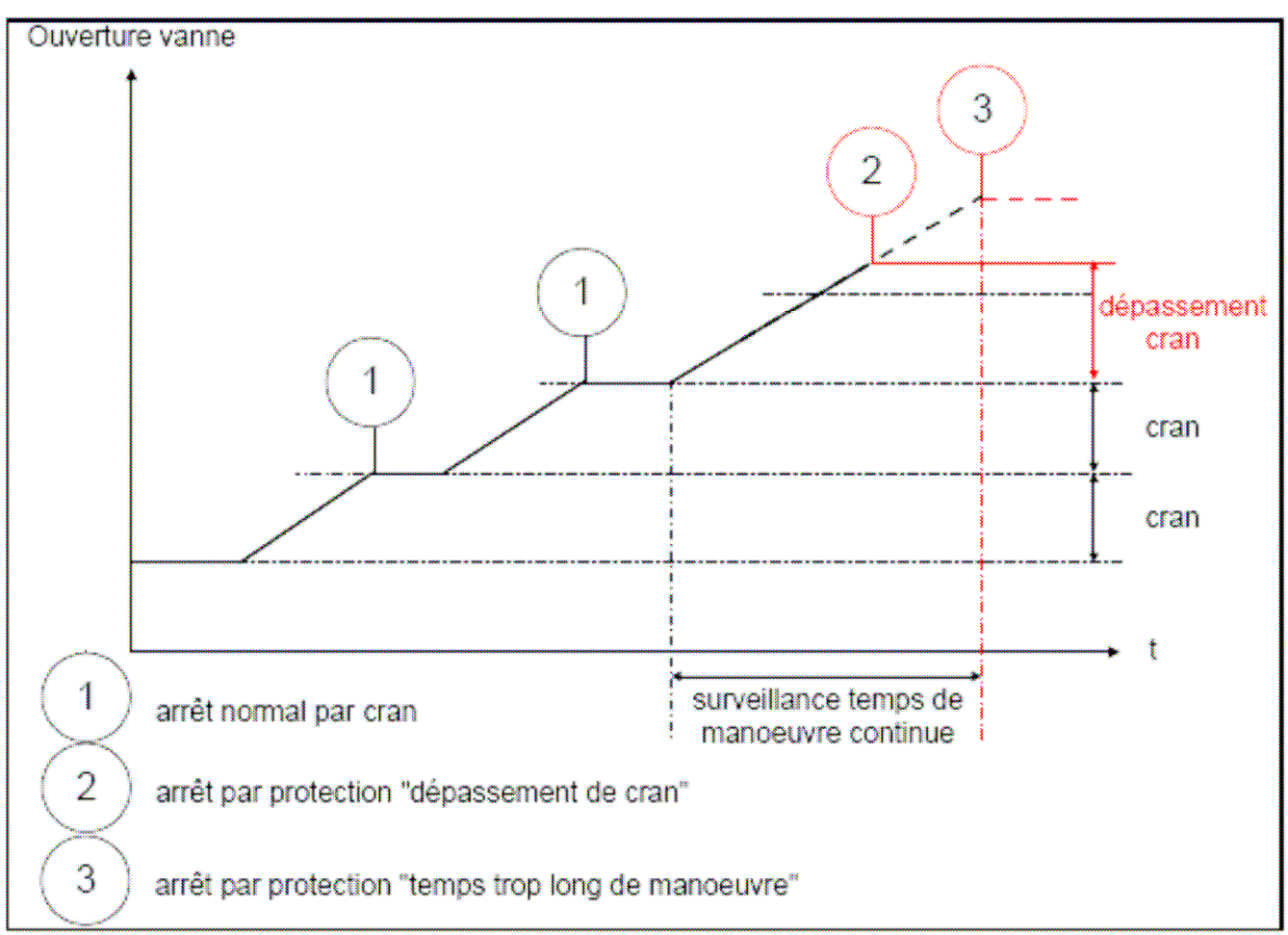

Figure 3.16 - Protections associées au dispositif de crantage

\subsection{Indication de position}

Pour assurer la maîtrise de la manœuvre, chaque point de commande manuelle doit disposer d'un indicateur de position de chacun des organes commandés :

- au droit de l'organe: l'indication pour la commande manuelle locale doit être opérationnelle du moment où la manœuvre de la vanne associée est possible (énergie de manœuvre) ; elle doit être à demeure et constamment visible pour l'opérateur depuis le point de commande (la règle mécanique peut éventuellement remplir cette fonction si elle est visible depuis le point de commande) ;

- au lieu de la commande centralisée : il est recommandé de disposer de deux chaînes de mesure et de deux indications de position indépendantes entre elles (l'utilisation d'un afficheur commun entre passes peut par exemple constituer le deuxième afficheur).

\subsubsection{Commandes hydrauliques à partir de la retenue}

Bien que le système de commande individuel par vanne assure un meilleur comportement d'ensemble puisque les risques de défaut sont répartis sur plusieurs vannes, ce genre de vannes ne permet pas d'éviter, à coup sûr, une non ouverture ou une manœuvre intempestive. Pour ce type d'ouvrage, il convient donc d'évaluer :

- le risque de non ouverture (par exemple obstruction de l'admission d'eau, blocage mécanique, gel des flotteurs...); 
- le risque d'ouverture intempestive (par exemple sur défaut d'étanchéité du flotteur, sur augmentation des fuites alimentant la chambre à flotteur...) ;

- les risques et les enjeux liés à la maîtrise de la variation des débits à l'aval, qui peuvent amener à reconsidérer la pertinence de ce type d'ouvrage.

Des dispositifs peuvent être mis en place pour détecter, dès leur apparition, des défaillances dont les conséquences peuvent s'avérer désastreuses pour la sécurité des tiers ou l'environnement si des mesures correctives ne sont pas prises rapidement. On citera principalement :

- détection de «flotteur noyé » : un capteur de pression embarqué sur le flotteur peut être installé à demeure pour contrôler en permanence si l'effort de manœuvre n'excède pas le pouvoir de flottabilité du flotteur. Un détecteur de seuil réglable déclenche une alarme (après avoir laissé le temps aux joints de se décoller) ;

- détection d'organe évacuateur coincé fermé : la combinaison d'une mesure de niveau située dans le puits à flotteur et de la position fermée permet de contrôler si l'ouverture est bien effective pour une hauteur d'eau dans la chambre correspondant à la mise en mouvement du système. Cette protection ressemble à la précédente, mais elle présente l'avantage de contrôler réellement la non ouverture de l'organe en cas de rupture de la chaîne cinématique ;

- détection d'organe évacuateur coincé ouvert : le principe consiste à contrôler si l'organe évacuateur s'est bien refermé au moment où le niveau dans le puits à flotteur est redescendu au-dessous du seuil prédéterminé.

Pour ce type d'ouvrage, à partir d'un certain débit, correspondant par exemple au tiers du débit d'équipement, il est souhaitable de prévoir une surveillance humaine de l'écoulement.

Dans certains cas, si l'ouverture intempestive est redoutée, on peut utiliser un verrouillage mécanique de l'organe en position fermée :

- il convient alors de respecter certaines dispositions concernant l'emploi de verrous sur les vannes d'évacuation de crues (cf. § 3.3.1.1.2);

- toutefois, lorsque la fonction principale de l'organe est d'assurer la tenue de la cote à une valeur donnée (généralement à $\mathrm{RN}$ ), le verrouillage des clapets est contre-indiqué, ces organes étant par ailleurs souvent prévus pour assurer une sécurité intrinsèque de l'ouvrage en cas d'élévation anormale du niveau de la retenue.

\subsubsection{Acquisition des informations}

L'exploitation d'un barrage fait généralement appel à des mesures de niveau, de débit ou de position et nécessite dans tous les cas d'acheminer des alarmes vers les exploitants. La fiabilité des moyens de détection et de transmission est donc essentielle.

\subsubsection{Mesures nécessaires à l'exploitation}

Les informations indispensables pour assurer, en toute situation, l'exploitation du barrage (surveillance de l'ouvrage, conduite automatique ou manuelle du barrage et des organes réglants) doivent répondre à cinq impératifs, à moduler en fonction de la complexité et de la dangerosité de l'ouvrage, et de son système de contrôle commande :

- emploi de matériel robuste et éprouvé ; 
- doubles points de mesure totalement indépendants (implantation, alimentation, cheminements, voies de transmission le cas échéant...) ;

- visualisation sur dispositifs autonomes et indépendants de l'automate de conduite quand il existe ;

- surveillance de la disponibilité de la mesure (défaut capteur, défaut tranche, défaut transmission le cas échéant...) ;

- surveillance de la cohérence des mesures physiques (écart entre les mesures comparé à un seuil).

Pour les mesures éloignées, on veillera à ne pas transmettre une mesure importante par deux voies présentant des modes communs de télétransmission (équipement, support, ...).

Pour les mesures d'aide à l'exploitation (par exemple débit d'un aménagement amont n'entrant pas dans la consigne de crue...), un seul point de mesure peut être suffisant.

\subsection{Mesures de niveau}

On veillera particulièrement :

- à tenir compte pour les capteurs de niveau des éventuelles particularités dynamiques du plan d'eau : basculement rive gauche/rive droite ou amont/aval ;

- à différencier les technologies de mesure afin de pallier les modes communs de défaillance (gel, envasement, ...) ;

- pour les capteurs de niveau par mesure de pression (bulle à bulle), à séparer très nettement les prises de chaque capteur et leur alimentation en air.

La sécurité de la conduite de l'ouvrage en crue nécessite que l'exploitant connaisse la cote du plan d'eau, qui au minimum doit pouvoir se lire sur une échelle graduée implantée dans la retenue.

II faut être vigilant à ce que cette échelle soit positionnée dans des zones abritées du courant principal (il ne faut pas que les corps flottants puissent l'arracher) et pas trop près de l'évacuateur de crues afin de pouvoir mesurer un niveau dans une zone où les vitesses sont faibles.

Les chiffres doivent être lisibles et pouvoir être éclairés par un projecteur. L'échelle doit être graduée sur l'ensemble de la plage de marnage autorisée dans la consigne d'exploitation et au minimum jusqu'au niveau maximal possible.

\subsection{Mesures de position}

Pour les organes pilotés en manuel depuis l'armoire de $1^{\mathrm{er}}$ rang, un seul point de mesure de position pourra être suffisant.

Pour les organes pilotés en manuel à distance ou en automatique, deux points de mesure de position sont souhaitables.

La fiabilité des prises d'information pour les mesures de position doit être soignée. La mesure physique se fait au plus près de l'organe, de préférence sur celui-ci, par opposition aux mesures intermédiaires de l'actionneur (treuil à chaîne ou à câble, moteurs, réducteurs). En particulier, l'installation des codeurs de position sur les clapets doit être étudiée avec soin car des difficultés ont souvent été rencontrées.

Des fonctions différentes (mesure de position 1, mesure de position 2, crantage...) imposent des prises d'information différentes pour limiter les modes communs. 


\subsubsection{Traçabilité}

Il est recommandé que la traçabilité des mesures nécessaires à la reconstitution de l'exploitation (au minimum, niveau de la retenue, position des vannes) soit assurée.

\subsubsection{Retransmission des alarmes}

L'acheminement des alarmes urgentes, ultimes sécurités de l'ouvrage pour l'intervention du personnel d'astreinte, doit présenter des garanties de fonctionnement élevées (robustesse aux défaillances, disponibilité et détectabilité de la perte de la fonction).

Les principales recommandations techniques qui s'imposent dans ce domaine sont les suivantes :

- utiliser des matériels de télétransmission fiables et éprouvés ;

- mettre en œuvre une organisation robuste de l'exploitant à la hauteur des enjeux (astreinte, compétences,...).

\subsubsection{Système de surveillance et de conduite}

Un système de surveillance et de conduite automatique d'un évacuateur de crues comprend obligatoirement trois composantes :

- un automate principal de surveillance et de conduite;

- un ensemble de dispositions externes à l'automate ;

- un dispositif de sauvegarde assurant la sécurité ultime.

Selon l'importance et le type d'évacuateur considérés, ces trois éléments sont plus ou moins développés mais examinés systématiquement.

Il est important de signaler que l'automate principal et le dispositif de sauvegarde sont deux systèmes distincts qui ne doivent pas être considérés comme assurant une redondance fonctionnelle de l'un par rapport à l'autre. Le dispositif de sauvegarde constitue une sécurité pour l'exploitation de l'ouvrage et son domaine d'action est distinct de celui de l'automate principal (cf. fig. 3.17, synoptique des zones de fonctionnement ci-dessous) : 


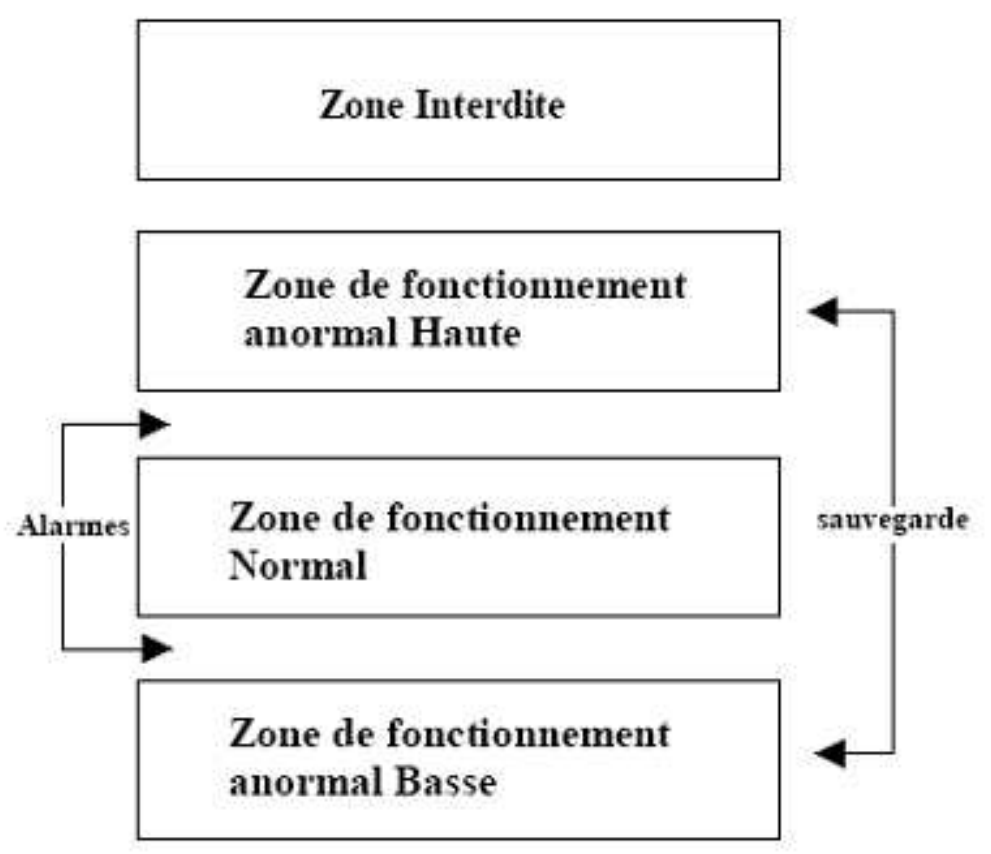

Figure 3.17 - Zones de fonctionnement de l'automate principal et de l'automate de sauvegarde

\subsubsection{Automate programmable de conduite du barrage}

Les organes du barrage sont normalement commandés par l'automate programmable de conduite du barrage.

Cet automate de conduite est situé au barrage pour éviter de passer par un système de télétransmission qui diminue la fiabilité de la conduite automatique. Il est implanté à une cote supérieure à la cote maximale atteinte lors de la crue de projet (PHE).

Cet automate doit posséder quatre catégories de fonctions, qui doivent être traitées de manière indépendante et qui sont développées dans les paragraphes suivants:

- la surveillance de l'aménagement ;

- la conduite proprement dite du ou des évacuateurs ;

- le contrôle et la surveillance de la conduite ;

- les contrôles de vraisemblance des ordres à émettre avec l'état du barrage.

Par ailleurs, des contrôles internes à l'automate doivent permettre de détecter des pannes matérielles et logicielles. Les conditions de retombée du " chien de garde » de l'automate sur défaut interne (défaut carte de communication, carte d'entrée, ...) doivent être évaluées en fonction des risques pour la conduite.

\subsection{Surveillance de l'aménagement}

Cette fonction intègre a minima les modules suivants :

- acquisition, traitement et validation des informations nécessaires à la surveillance ;

- traitement des alarmes ;

- élaboration de l'alarme «état de veille »;

- élaboration de l'alarme « état de crue ». 


\subsection{Conduite du ou des évacuateurs}

Cette fonction peut intégrer les modules suivants :

- acquisition, traitement et validation des informations ;

- calcul des valeurs de niveau filtrées ;

- conversion des hauteurs mesurées en débit ;

- calcul de la consigne de niveau à l'amont du barrage ;

- calcul de la consigne de débit à évacuer par le barrage ;

- répartition de la consigne de débit au barrage entre les vannes inférieures et supérieures ;

- calcul des consignes unitaires de position des vannes supérieures et inférieures ;

- envoi des ordres de commande aux vannes supérieures ;

- envoi des ordres de commande aux vannes inférieures.

Les périodes d'envoi des ordres et leur amplitude sont choisies de manière à être compatibles avec les gradients maximaux de débit entrant.

L'automate programmable de barrage doit interdire le démarrage simultané de deux organes en conduite automatique.

La conduite d'un barrage est souvent exprimée en régulation de niveau. La régulation devra être à la fois :

- assez réactive pour ne pas provoquer des variations de niveau amont inacceptables ;

- assez souple pour optimiser le nombre de manœuvres des organes de crue.

Celle-ci doit faire l'objet de simulations permettant de vérifier que les objectifs recherchés sont respectés.

\subsection{Contrôle et surveillance de la conduite}

L'automate programmable de barrage assure en permanence le contrôle et la surveillance de la conduite de l'aménagement.

Dans un premier temps, il donne l'alerte aux agents d'astreinte.

S'il détecte une anomalie importante, l'automate lui-même décide de se retirer de la conduite, l'état de l'aménagement étant alors figé jusqu'à intervention du personnel d'astreinte. Dans ce cas, le dispositif de sauvegarde reste toutefois en action. Les contrôles effectués par l'automate programmable portent notamment sur:

- la validité des mesures des capteurs d'entrée (alimentation, seuil, gradient, invariance, cohérence d'informations doublées...) ;

- l'état des organes de crue (alimentation, défaut propre, ...).

La surveillance concerne les valeurs des paramètres principaux par rapport aux consignes ou d'autres mesures sur l'état de l'aménagement (niveau amont, débit amont/débit aval). On peut citer :

- contrôle du respect du niveau maximum et minimum autorisé suivant le type d'exploitation ;

- contrôle du respect d'un débit minimum ; 
- contrôle du respect du gradient de débit à l'aval de l'aménagement ;

- contrôle de la cohérence de l'état des organes de crue par rapport à l'état de l'aménagement.

\subsection{Contrôles de vraisemblance des ordres}

Ces contrôles servent à s'assurer du bon fonctionnement des organes de crue. Les informations obtenues sont à émettre avec les informations sur l'état du barrage. On peut citer :

- contrôle du sens de manœuvre de l'organe de crue par rapport à l'ordre émis ;

- contrôle de la variation de la mesure de position de l'organe de crue par rapport à l'ordre émis ;

- contrôle du temps trop long de manœuvre de l'organe de crue ;

- contrôle de l'absence de mouvement de l'organe de crue en dehors de l'émission d'ordres ;

- contrôle de la dérive de l'organe de crue (commande hydraulique).

\subsubsection{Dispositif de sauvegarde}

Le dispositif de sauvegarde est un équipement totalement indépendant de tous les autres matériels (principaux). II intervient dès lors que les mesures de référence s'écartent significativement des plages normales d'exploitation. II fait partie des " sécurités intrinsèques » de l'ouvrage.

C'est le dispositif ultime par rapport à la sécurité d'exploitation et des personnes. II peut suivant les cas assurer les fonctions suivantes :

- uniquement alerter ;

- alerter et bloquer la conduite automatique en attente d'une intervention humaine ;

- alerter et se substituer à la conduite automatique pour mettre l'ouvrage en sécurité en attente d'une intervention humaine. Dans ce dernier cas, on parle d'automatisme de sauvegarde (AS).

\section{La mise en œuvre d'une fonction de sauvegarde agissant sur les organes de crues appelle les remarques suivantes:}

- le but d'un dispositif de sauvegarde agissant sur les organes de crues est, en attendant l'arrivée de l'exploitant, de stopper l'exhaussement (ou l'abaissement) du niveau et non de le ramener à sa valeur initiale de consigne, pour ne pas créer de sur-débits à l'aval. Son action est donc limitée dans le temps ;

- on peut noter les inconvénients d'un automatisme de sauvegarde :

- actions commandées par des seuils de niveau situés dans des puits extérieurs, donc accessibles (vandalisme),

- difficulté à cohabiter avec les contraintes aval,

- absence de garde-fou en cas de dysfonctionnement interne, du fait de sa rusticité.

En conséquence, la mise en œuvre d'un dispositif de sauvegarde est à considérer avec précautions et il conviendra de privilégier, lorsque cela est possible, un dispositif de sauvegarde se limitant à l'appel de l'astreinte. À ce titre, les alarmes urgentes, ultimes sécurités de l'ouvrage, doivent présenter des garanties de fonctionnement extrêmement 
élevées, notamment en intégrant les dispositifs de transmission et de diffusion des messages d'alarmes.

Une étude très précise doit être effectuée dans tous les cas, de manière à justifier la sécurité apportée par le dispositif de sauvegarde retenu.

\subsection{Suivi et maintenance}

\subsubsection{Principes généraux}

\section{Une conception cohérente et sécuritaire des équipements électromécaniques d'un barrage ne peut garantir à elle seule la sécurité de l'ouvrage dans la durée.}

Une maintenance régulière des équipements de l'ouvrage et des organes de secours est en effet indispensable. Le maintien en condition opérationnelle des installations doit également être assuré par une surveillance, des contrôles et des essais périodiques.

La surveillance de routine du barrage doit être effectuée lors de tournées d'inspection par le personnel exploitant, selon une périodicité d'une semaine à un mois en fonction de l'importance du barrage. Chaque barrage doit faire l'objet d'une liste de points à surveiller. Une inspection visuelle plus approfondie peut être programmée périodiquement (une fois par an ou tous les deux ans) ou après un événement exceptionnel tel qu'une forte crue ou un séisme.

Des essais périodiques doivent être réalisés sur le barrage. Ceux-ci peuvent concerner les vannes et clapets en ouverture totale ou partielle, les capteurs et leurs liaisons avec l'exploitant (ou l'automate de commande), l'alimentation électrique de secours (groupe électrogène) et d'ultime secours. Chaque essai doit faire l'objet d'un mode opératoire (conditions de l'essai, critères à vérifier), d'un compte-rendu d'essai et d'instructions écrites et précises pour l'exploitant.

Des contrôles périodiques ainsi que les entretiens doivent être réalisés sur la base des consignes de fonctionnement et d'entretien qui doivent également être fournies par les constructeurs des équipements. II est important de rappeler que ces consignes doivent être demandées dans le cahier des charges, et qu'il est impératif de les recevoir sous une forme exploitable.

On notera également que les consignes des constructeurs en matière de maintenance et d'essais sont parfois ignorées ou reportées, car contraignantes pour les exploitants. II est pourtant impératif que les recommandations des constructeurs et fournisseurs soient respectées ou adaptées en toute connaissance de cause. De plus, et si possible, le choix des équipements de manœuvre devra intégrer la possibilité de leur maintenance.

\subsubsection{Essais périodiques des organes}

La vérification périodique du fonctionnement en réel d'un organe de sécurité est un des moyens les plus sûrs pour contrôler son aptitude à fonctionner.

Pour les évacuateurs de crues, les organes et matériels importants pour la sûreté hydraulique à essayer sont : 
- les vannes ou les clapets :

- l'essai peut être partiel ou total. La périodicité est à adapter en fonction des risques à l'aval, du nombre de vannes, de la complexité des mécanismes, des gradients de débits, du gel. Lorsque le nombre de manœuvres est important entre deux essais, on peut se dispenser de l'essai prévu,

- l'essai d'ouverture totale peut aussi être fait lorsque les vannes sont hors d'eau, bien que cette manœuvre détériore les joints. II est opportun de profiter des vidanges totales pour essayer à ouverture complète les évacuateurs de fond ou de demi-fond,

- pour les vannes manœuvrées par treuils à chaînes, les très hautes pressions diamétrales des axes sur les maillons engendrent des difficultés de graissage et le grippage de ce type de chaîne est fréquent. II est nécessaire de prévoir un entretien régulier qui consiste en un graissage soigné et en une manœuvre complète de la chaîne pour conserver sa "souplesse ". Le fascicule de recommandations techniques français du programme LUBAQUA (STCPMVN, 1993) recommande, si possible, une manœuvre complète toutes les 4 à 8 semaines des tronçons immergés,

- ce dernier point n'est pas toujours possible et il n'est pas rare de voir des chaînes « rigidifiées » par le grippage et la corrosion. Dans ces conditions, et en cas d'utilisation de telles chaînes, des dispositions constructives doivent être prises (bagues autolubrifiantes, chaînes inox, etc.) pour permettre de réduire les fréquences d'entretien ;

- les mesures des niveaux et les alarmes signalant les défauts de matériels : l'essai est fait en réel en agissant sur le capteur pour vérifier la liaison avec l'exploitant et la qualité de l'information ;

- les alimentations de secours et d'ultime secours :

- les systèmes de permutation normal-secours d'alimentation, ainsi que les groupes électrogènes de secours, s'ils existent, doivent être essayés en réel; les commandes manuelles (manivelles, pompes) ou leurs équivalents (moteur thermique) doivent être aussi essayés,

- ces essais sont à faire en charge, de préférence avant une période de risque de crues. On peut, par exemple, profiter des fins de crues pour manœuvrer l'évacuateur de crues par la source de secours ;

- les dispositifs de sauvegarde: ces dispositifs ayant pour rôle d'agir dans des conditions extrêmes (de cote et parfois de débit), il n'est pas toujours possible, voire raisonnable, de réaliser des essais en conditions réelles. Des essais périodiques doivent toutefois être réalisés, par action sur les capteurs, pour vérifier l'ensemble de la chaîne fonctionnelle (liaison avec l'exploitant et qualité de l'information, action sur les vannes...).

\subsubsection{Contrôles périodiques}

Si les essais périodiques permettent de s'assurer de la sécurité de fonctionnement des organes évacuateurs, les contrôles périodiques permettent de caractériser l'état des différents constituants des vannes et clapets dans le but d'en effectuer l'entretien ou le remplacement.

Que ce soit à la construction d'un ouvrage neuf ou après toute opération de maintenance (rénovation, remplacement), une liste des contrôles périodiques en exploitation de l'ensemble des ouvrages, matériels et systèmes doit être proposée par le maître d'œuvre. Après validation, ceux-ci sont ensuite intégrés par le maître d'ouvrage dans les opérations de maintenance du barrage et de ses différents équipements.

Ces contrôles doivent faire l'objet d'un compte-rendu d'essais. 


\subsubsection{Opérations de maintenance}

Compte tenu des enjeux de sûreté, la maintenance préventive conditionnelle est la mieux adaptée à ce type d'ouvrage, en mettant en évidence suffisamment à l'avance les risques d'incidents prévisibles et en permettant d'optimiser les coûts d'entretien. Le principe de la maintenance corrective systématique effectuée après un incident est à proscrire, puisque le matériel serait alors indisponible à une période où il doit servir, diminuant ainsi la sécurité du barrage.

Par ailleurs, il faut prêter la plus grande attention à la période choisie pour la maintenance lourde des vannes et éviter les périodes de crue. De même, toute opération sur des matériels assurant une fonction de sécurité doit faire l'objet d'une analyse de risques. En effet, l'indisponibilité générée sur l'organe ou le matériel dégrade les marges de sécurité. L'analyse devra prendre en compte les moyens d'anticipation sur les risques redoutés (et leur fiabilité), l'identification des conditions pour lesquelles la sûreté d'exploitation peut être compromise, les dispositions compensatoires et la conduite à tenir en cas de dépassement de ces conditions. Il est souhaitable que cette analyse soit régulièrement actualisée durant l'opération, les hypothèses prises pouvant évoluer dans le temps.

\subsubsection{Requalification des ouvrages et matériels}

Il convient de porter la plus grande précaution après toute intervention sur les matériels de sécurité.

Des requalifications doivent être réalisées chaque fois que des opérations ont eu lieu sur un matériel. Cela vaut aussi bien suite à des opérations de maintenance spécialisée que de maintenance courante.

Les démarches de requalification doivent systématiquement être menées lors de toute opération ayant un caractère intrusif sur les ouvrages ou matériels.

\subsection{Utilisation des évacuateurs de crues - Gestion du passage des crues}

La fiabilité du système d'évacuation des crues nécessite que les organes eux-mêmes soient fiables (les dispositions constructives et les modalités de maintenance présentées ci-avant participent à cet objectif), mais les modalités d'utilisation de ces organes en crue demeurent également essentielles.

Des consignes précises sont nécessaires afin de prévoir toutes les situations possibles, sachant que les règles d'exploitation de tels ouvrages sont d'abord gouvernées par le respect de la sécurité des tiers. L'objectif essentiel de ces consignes est d'éviter les prises de décision à chaud et les improvisations qui pourraient être nuisibles à l'efficacité et à la sécurité, en ayant défini la conduite à tenir à l'avance, tenant compte des préoccupations des différents intervenants. La réglementation issue du décret 2007-1735 du 11 décembre 2007 impose l'existence de ces consignes pour tous les barrages, les consignes des classes A, B et $C$ étant approuvées par l'État ; ceci marque toute l'importance des consignes et de leur mise en œuvre pour garantir la sécurité des ouvrages lors des épisodes de crues, qu'il s'agisse de crues assez fréquentes ou des crues extrêmes. 
Les critères d'exploitation retenus résultent toujours d'un compromis entre la sécurité à l'amont du barrage et celle à l'aval.

La gestion des barrages peut être sectorisée en trois états, définis en fonction de la situation hydrologique (BETCGB, 2002) :

- l'état d'exploitation normale, pour lequel il n'y a pas de suivi particulier de la situation hydrologique ;

- l'état de veille, qui définit le niveau de mobilisation de l'exploitant, les essais et les contrôles à effectuer. Cet état implique le contrôle régulier de l'évolution des paramètres hydrauliques et hydrologiques (débits, niveaux, précipitations...). Cet état est prononcé par le personnel d'exploitation à partir de conditions prédéfinies (souvent seuil reliant le débit entrant et le niveau de la retenue), éventuellement déclenché par un automatisme de surveillance. Au cours de l'état de veille, des essais et manœuvres peuvent être réalisés afin de s'assurer qu'en cas de crue, les éléments garantissant la sécurité sont en état de fonctionner. Ces essais permettent notamment de diminuer les risques de dysfonctionnement de l'évacuateur de crues, et notamment les risques de blocage complet des vannes ;

- l'état de crue, qui est prononcé lorsque certains paramètres suivis pendant l'état de veille atteignent une valeur seuil. La surveillance permanente du barrage est alors nécessaire. Cet état coïncide souvent avec les premières manœuvres sur les organes d'évacuateur de crues.

Les manœuvres d'exploitation prioritaires sont celles liées au passage des crues. Dans ce but, il faut :

- adapter les règles d'exploitation aux problèmes rencontrés selon le type d'ouvrage (notamment pour les barrages en enrochement ou en terre), le site, l'environnement ;

- respecter les règles d'exploitation malgré les défaillances éventuelles de matériel ;

- adapter le degré de sécurité aux conséquences potentielles des défaillances (notamment en prenant en compte les contraintes aval propres à chaque cas).

La conduite automatique présente des avantages sensibles pour les évacuateurs des barrages de basse chute.

En revanche, pour les barrages de grande hauteur à réserve importante, l'intérêt de la conduite automatique est plus délicat. Pour ces ouvrages, l'exploitation en période de crues doit se faire en présence de personnel, soit manuellement, soit en automatique jusqu'à un débit précisé dans la consigne d'exploitation.

Si l'évacuateur est constitué d'un déversoir libre ou si l'ouvrage est pourvu de vannes automatiques dont une défaillance n'aurait pas de conséquence sur la sécurité des personnes, la présence humaine au barrage en crue n'est pas nécessaire à la gestion de l'ouvrage mais reste hautement souhaitable, ne serait-ce que pour assurer une fonction d'alerte en cas de complications.

Pour tout ouvrage présentant un enjeu pour la sécurité des tiers, il faut envisager, en complément de l'automatisme normal de manœuvre, lorsqu'il existe, des sécurités indépendantes et un dispositif de sauvegarde, sachant qu'une intervention humaine est indispensable soit en cas d'anomalie, soit à partir de certaines configurations d'exploitation.

Les consignes de crue se présentent généralement sous la forme de deux documents, la consigne générale d'évacuation des crues (CGEC) et la consigne d'exploitation en crue (CEC), dont le contenu est défini ci-après. Pour des ouvrages simples (par exemple, ceux ne disposant pas d'évacuateurs vannés), il est acceptable de fusionner ces deux documents. 


\subsubsection{Consigne générale d'évacuation des crues}

La consigne générale d'évacuation des crues est un document établi par l'exploitant, en concertation avec les différents services de l'État ou des autorités concernées qui agissent au titre d'organisme de contrôle. Elle doit être approuvée par la personne responsable de la sécurité civile, en France, le préfet. Elle a pour objectif de définir les contraintes et de hiérarchiser les objectifs à respecter vis-à-vis de la sécurité propre du barrage et vis-à-vis de son environnement amont et aval. Elle précise également les liaisons à assurer avec les services et autorités concernés par les crues.

Les contraintes dépendent des caractéristiques de l'ouvrage (barrage, évacuateur de crues et retenue), du cours d'eau, de l'environnement amont et aval et des autres usages du cours d'eau.

Les objectifs sont fixés par l'exploitant et peuvent être classés en plusieurs catégories selon qu'ils visent à :

- assurer la sécurité du barrage pendant la crue ;

- assurer la sécurité des tiers : être transparent en période de crues, retarder la montée des eaux à l'aval et écrêter les crues ;

- répondre aux préoccupations de l'exploitant : assurer le remplissage maximal de la retenue, effectuer des chasses de dégravement.

La consigne générale d'évacuation des crues doit définir de façon précise les informations à fournir, les services ou autorités auxquelles les informations sont fournies, la périodicité de fourniture des informations, les moyens de transmission de l'information, et la personne chargée de transmettre l'information.

\subsubsection{Consigne d'exploitation en crue}

La consigne d'exploitation en crue est également établie par l'exploitant et approuvée par le préfet.

Elle définit les conditions de passage en état de veille et en état de crue, à partir de paramètres définis (débit amont ou entrant dans la retenue, mesuré ou estimé, cote de la retenue, vitesse de variation du débit et/ou de la cote, précipitations, etc.).

Pour les ouvrages vannés, cette consigne précise les règles de déstockage pendant toute la durée de la crue, c'est-à-dire pendant la montée de crue mais également en décrue. Les règles exposées font généralement appel aux mêmes paramètres que ceux utilisés pour la définition de l'état hydrologique. Ces règles sont généralement précisées sous forme d'abaques ayant pour abscisse la cote de la retenue et pour ordonnée la variation de cote pendant le pas de temps de scrutation (en $\mathrm{cm}$ par $1 / 2 \mathrm{~h}$ par exemple) ou le débit entrant ou encore le débit stocké. La mise en œuvre de la consigne peut s'appuyer sur des calculateurs mais doit pouvoir être assurée «à la main » (et donc par exemple avec une version papier des abaques).

L'établissement de la consigne d'exploitation des évacuateurs vannés impose une vérification des vitesses d'ouverture des vannes, qui ne doivent pas être trop lentes pour éviter d'être dépassé par la crue, ni trop rapides au risque de générer des sur-gradients de débits préjudiciables pour les avalisants. 
La consigne traite aussi de la conduite à tenir en cas de crues avec complications (lien avec un PPI éventuel, possibilité d'utiliser les autres organes hydrauliques que les évacuateurs de crues, etc.), des rapports à établir après la crue...

\subsubsection{Régulation}

Afin de maintenir le niveau de la retenue, parfois constant, prévu dans la consigne, une régulation est nécessaire par la commande des vannes. II s'agit de respecter cet objectif sans provoquer de sur-niveaux de la retenue ni de sur-débits à l'aval, les deux étant potentiellement dangereux.

On emploie classiquement une régulation type PID (Proportionnel, Intégral, Dérivé), qui ajuste le débit lâché en fonction des variations du niveau de la retenue (proportionnel) et de l'écart à la consigne (intégral). Un terme tenant compte du débit entrant dans la retenue améliore la régulation. Ce type de régulation est en général suffisant pour les grandes retenues avec beaucoup d'inertie. Dans tous les cas, une fonction de régulation de niveau doit faire l'objet de simulations permettant de vérifier que les objectifs recherchés sont respectés.

En cas de besoin d'une régulation fine (petite retenue, forte variation des débits entrants, etc.), une régulation plus précise peut être obtenue avec des modèles prédictifs embarqués sur site. C'est en particulier souvent le cas pour les barrages mobiles en rivière.

\subsubsection{2 Équi-ouverture}

Il est important d'analyser la séquence d'ouverture des vannes. Le plus souvent, il conviendra de favoriser l'équi-ouverture des vannes, pour une bonne dissipation de l'énergie à l'aval. La non équi-ouverture peut provoquer des affouillements à l'aval des vannes, l'énergie à dissiper y étant plus importante. Toutefois, ce point doit justifier une analyse de risques au cas par cas.

Ainsi, pour augmenter le débit, il faudra ouvrir d'un seul pas la vanne la moins ouverte ; pour le réduire, il faudra fermer d'un seul pas la vanne la plus ouverte. Ainsi, toutes les vannes auront la même ouverture à un pas près.

\subsection{Retours d'expérience sur des cas de défaillance}

Les cas de défaillance mentionnés ci-dessous, basés sur des cas réels, invitent à apporter une grande vigilance à la conception et à l'utilisation des équipements pour éviter les défaillances pouvant se révéler catastrophiques ou plus simplement les complications liées aux modifications à réaliser dans des conditions souvent délicates d'exploitation des ouvrages.

L'attention est attirée sur l'augmentation importante des risques de défaillance dès lors que les conditions hydrologiques et météorologiques qui leur sont liées s'approchent des valeurs exceptionnelles voire extrêmes; par ailleurs, ne sont pas ici abordés des exemples de défaillance résultant d'erreurs humaines, dont le risque de survenance, notamment lié aux situations de stress que peuvent connaître les différents opérateurs, peut être très fortement majoré à l'occasion des crues majeures. 


\subsubsection{Défauts de conception}

Les défaillances liées aux défauts de conception proviennent fréquemment, non pas de la tenue des structures dont le calcul est souvent bien maîtrisé, mais des organes de manœuvre qui sont parfois mal adaptés ou mal calculés en raison des difficultés d'appréhension des efforts et charges «parasites » liés, par exemple aux déformations des équipements et aux efforts de manœuvre. De nombreux cas de défaillances de ce type ont été relevés sur les ouvrages évacuateurs. Les principaux organes défaillants sont les suivants :

- rupture des freins et/ou des accouplements des treuils de manœuvre (erreur de calcul ou matériaux défectueux) ;

- rupture des accouplements des arbres de synchronisation suite à un blocage de l'organe manœuvré ;

- rupture des pignons de treuil (efforts sous-estimés, mauvaise conception et/ou réalisation des treuils) ;

- ruptures de chaînes ou de brimbales (efforts sous-estimés) ;

- rupture des bras d'une vanne segment (conception trop légère ${ }^{6}$ ) ;

- rupture des articulations inférieures de clapets (modification des articulations, erreur de calcul) ;

- ouverture intempestive de clapets (rupture de tuyauteries, schéma hydraulique inadapté, rupture de l'étanchéité de fond du vérin) ;

- rupture de la structure par sollicitation d'un mode propre de vibration dans des conditions particulières d'écoulement (HYDRO, 2009).

II n'est également pas rare de rencontrer, lors des essais de mise en service, des vannes de fond qui ne ferment pas sous leur propre poids alors qu'elles sont conçues pour cette fonction. Le calcul de ces vannes n'est pas particulièrement complexe, mais il nécessite une bonne appréhension des efforts liés à la position du couteau de la vanne, de la rigidité de sa traverse frontale, des effets dynamiques de la lame d'eau sur le seuil de la vanne et de l'effet de la poussée d'Archimède dans le cas d'une vanne à étanchéité aval et bordé amont.

On a rencontré également le cas de vannes segments de grandes dimensions (largeur $22 \mathrm{~m}$, hauteur 14,25 m) installées sur un seuil, fléchissant au niveau du couteau sous la charge statique et créant un déplacement horizontal de celui-ci de quelques centimètres au centre de la vanne. Cette déformation, qui était acceptable d'un strict point de vue mécanique, a engendré une fuite au centre de la vanne due à l'inclinaison du seuil. La pression chutant sous l'influence de la fuite, la vanne reprenait sa position initiale puis se déformait de nouveau, créant ainsi une « respiration » inacceptable.

Enfin, on constate également la difficulté d'appréhender les efforts de manœuvre, particulièrement sur les clapets déversants où les conditions hydrauliques et les corps flottants peuvent sensiblement faire varier les charges sur les organes de manœuvre.

\subsubsection{Défauts des informations entrantes du système de gestion}

Les équipements hydromécaniques doivent souvent obéir à des consignes de gestion des ouvrages définies par l'exploitant en fonction d'objectifs fixés à l'avance. Des programmes sont aujourd'hui établis afin de gérer au mieux ces paramètres et permettent une bonne automatisation de la gestion de l'aménagement à l'aide d'automates, dont il est possible

\footnotetext{
${ }^{6}$ Barrage de Sainte-Marguerite, 1995
} 
d'assurer la redondance à un coût raisonnable (voir cependant les réserves émises sur cette automatisation en 3.3.2.1).

Les problèmes les plus fréquemment rencontrés sont liés à une mauvaise information ou, dans certains cas, à un manque d'information fiable en ce qui concerne les débits entrants, surtout en période de fortes crues. II n'est en effet pas rare de voir des stations de mesures de niveaux et/ou de débits situées en amont de la retenue détériorées par les crues ellesmêmes, privant l'automate de données essentielles pour la gestion des aménagements en crue. Également, le manque d'information lié à l'absence d'équipement peut engendrer des incidents plus ou moins graves. La solution à ces problèmes reste la fiabilisation des données à l'aide de plusieurs sources redondantes permettant la validation par l'automate des informations reçues et l'élimination des informations aberrantes ou erronées.

On notera par exemple l'incident survenu sur le barrage de Nang Béto au Togo, où la seule information de débit était donnée par la mesure sur l'ouvrage de la variation du plan d'eau dans le temps. Un orage violent s'étant abattu sur la retenue $(100 \mathrm{~mm}$ en une heure environ), la variation de niveau a été interprétée par l'automate comme une crue de fréquence décamillennale, ce qui a immédiatement conduit à l'ouverture automatique de l'ensemble des vannes (qui étaient initialement fermées) afin d'assurer l'amortissement de la crue. Sans l'intervention immédiate du personnel en charge de l'exploitation des ouvrages, les conséquences sur la population en aval du barrage auraient pu être dramatiques.

On peut aussi noter l'incident du barrage de La Croux (Tarn, 1984), où une crue artificielle a été générée suite à une série de dysfonctionnements avant requalification suite à des travaux de maintenance (clapets hors service, erreur de logiciel dans l'automate programmable, pas de commande des vannes augmentés à cause de vibrations aux petites ouvertures, dispositif de sauvegarde inopérant, etc.). Le retour d'expérience de cet incident a permis de poser les bases de la sécurité d'exploitation des barrages et notamment des précautions à prendre pour leur automatisation, dont de nombreuses dispositions sont reprises dans le présent document.

\subsubsection{Défaut de maintenance}

Les défaillances liées aux défauts de maintenance sont fréquemment recensées sur les ouvrages hydrauliques. Elles sont le plus souvent sans conséquence grave sur la conduite des équipements. Toutefois, elles peuvent avoir des conséquences dramatiques lorsqu'elles conduisent à la rupture d'un barrage tel que celui de Belci en Roumanie où un manque d'entretien des équipements a conduit en 1991 à la rupture de ce barrage en terre $\left(\mathrm{H}=18,5 \mathrm{~m}, \mathrm{~V}=12,7 \mathrm{hm}^{3}\right)$ et à la mort de 25 personnes (Diacon et al., 1992).

À l'amorce d'une crue importante, le gardien du barrage a commencé à ouvrir les quatre vannes. Dix minutes plus tard, alors qu'une vanne est ouverte, une panne de secteur stoppe l'ouverture des autres. Les batteries du groupe électrogène de secours ayant été retirées quelques jours auparavant pour secourir un camion, ce système de secours est inexploitable. L'ouverture complète à la main de la vanne entrouverte s'avère impossible car bloquée par un arbre. Cette succession d'avaries a entraîné le déversement par-dessus le barrage et l'ouverture de brèches, une de $112 \mathrm{~m}$ de longueur par $15 \mathrm{~m}$ de hauteur, l'autre de $40 \mathrm{~m}$ de longueur par $10 \mathrm{~m}$ de profondeur.

Ce concours exceptionnel d'événements défavorables n'est heureusement pas fréquent. II montre néanmoins l'importance de garantir le maintien de l'ensemble des équipements de sécurité d'un ouvrage en parfait état de fonctionnement. 
Plus couramment, les incidents recensés concernent principalement des défaillances liées à des négligences ou des difficultés au niveau de l'entretien. Quelques cas de défaillances rencontrés sont résumés ci-après:

- chaînes Galle rigidifiées par la corrosion ou grippage (obligation de remplacer les chaînes avant la manœuvre complète des vannes) ;

- accouplements moteur-réducteurs affaiblis par l'usure (risque de rupture de l'accouplement). On peut noter que tous les treuils en service actuellement ne sont pas encore équipés de double système de freinage, et la rupture d'un accouplement peut engendrer de graves accidents si le seul frein est par exemple intégré au moteur électrique ;

- organes de contrôle de position (fins de courses, capteurs de position, synchronisation) défectueux ou déplacés ;

- groupe électrogène défectueux, hors service ou mal entretenu (parfois simplement par manque de carburant...). 


\section{Recommandations pour le dimensionnement des évacuateurs de crues}

\subsection{Pratiques françaises actuelles pour le dimensionnement des évacuateurs neufs ou existants}

\subsubsection{Préambule}

La pratique française en matière de barrages et pour le dimensionnement des évacuateurs de crues en particulier repose sur des règles de l'art couramment appliquées sans que des textes à caractère normatif ou des guides officiels ne viennent l'imposer.

Les éléments indiqués dans ce paragraphe résultent notamment de positions communes prises par le Comité Technique Permanent des Barrages (devenu Comité Technique Permanent des Barrages et des Ouvrages Hydrauliques depuis décembre 2007) à l'occasion de l'examen de nombreux projets de grands barrages (selon la définition française, c'est-àdire intéressant la sécurité publique au sens de la circulaire du 14 août 1970 ou de classe $A$ depuis le décret 2007-1735). Pour les petits barrages, on peut faire référence au document publié par le Comité Français des Grands Barrages (CFGB, 1997), dont le champ d'application est défini comme celui des ouvrages de hauteur inférieure à $25 \mathrm{~m}$.

\subsubsection{Cote des PHE et revanche}

Comme indiqué au chapitre 1, la cote des PHE est au plus égale à la cote de retenue admissible (et vérifiée par une note de calcul) pour la structure. En outre, il est d'usage de prendre en compte une revanche minimale entre la cote des PHE et la crête.

Pour les barrages rigides (poids, voûtes, à contreforts, ...), la cote des PHE est au plus égale à celle de la crête, sauf pour les barrages déversant sur toute leur longueur (dans ce cas, la vérification se fait par rapport aux culées d'extrémité).

Pour les barrages en remblai, la revanche à prendre en compte intègre la hauteur des vagues de façon à limiter le franchissement de vagues sur le barrage. Ce dernier paramètre est habituellement calculé à partir de la forme de la retenue (le fetch), la vitesse des vents, l'inclinaison du parement, la présence éventuelle d'un pare-vague... Cette revanche sur les PHE peut conduire à adopter une cote de PHE plus faible que ce qu'autoriserait la simple vérification de la résistance de la structure.

\subsubsection{Crues de dimensionnement}

Pour les grands barrages en béton ou en maçonnerie, la crue de dimensionnement correspond souvent à une période de retour de 1000 ans. Pour certains barrages-poids, la crue de dimensionnement a parfois été fixée à une période de retour de 5000 ans.

Pour les grands barrages en remblais, la crue de dimensionnement correspond à une période de retour de 10000 ans. 
L'hydrologue fournit généralement au projeteur un débit de pointe, un débit moyen pendant l'épisode de crue et un hydrogramme. Dans certains cas, plusieurs hydrogrammes sont proposés, par exemple avec des durées de crue différentes.

\subsection{4 Écrêtement par la retenue}

Lorsque les capacités d'écrêtement par la retenue sont très faibles (volume de la crue notablement supérieur à la différence de volume de retenue entre la cote des plus hautes eaux et la cote de retenue normale), on néglige l'effet de l'écrêtement et l'évacuateur de crues est conçu pour « passer » le débit de pointe de la crue.

Dans le cas contraire, il est admissible de prendre en compte l'effet de l'écrêtement en faisant les hypothèses suivantes:

- retenue initialement à la cote de retenue normale ;

- $\quad$ prise en compte du volume de la crue (ce paramètre, très important lorsque l'écrêtement est pris en compte, doit dans ce cas faire l'objet d'une attention particulière dans l'étude hydrologique) ;

- $\quad$ prise en compte de la dynamique des phénomènes (forme de l'hydrogramme de crue et gradient de débit entrant, vitesse de manœuvre des évacuateurs vannés) ;

- prise en compte de la débitance des organes évacuateurs de crues et de sa variation en fonction de la cote de retenue ${ }^{7}$;

- non prise en compte des autres organes hydrauliques (prises d'eau usinières, vidange de fond). En particulier, les capacités d'évacuation de la vidange ne sont prises en compte que dans la mesure où cet organe est spécifiquement conçu pour assurer aussi une fonction d'évacuation des crues (donc avec les redondances nécessaires sur l'alimentation électrique, le mode de commande...) et où son usage est compatible avec les consignes d'exploitation en crues.

\subsubsection{Dimensionnement hydraulique de l'évacuateur de crues}

Le ou les organes prévus pour l'évacuation des crues doivent, a minima, être capables de faire transiter, en additionnant la débitance de chacun des organes ou des passes, le débit de pointe de la crue (éventuellement écrêté comme indiqué ci-dessus) pour une cote de retenue égale à celle des PHE. II n'est, sauf cas particulier, pas tenu compte d'un abattement pour indisponibilité partielle de certaines passes et l'évacuateur est considéré comme fonctionnant de façon nominale.

\subsubsection{Dimensionnement hydraulique des coursiers, organes de dissipation...}

L'ensemble de ces organes doit être conçu pour fonctionner sans entraîner ni de risques de surverse, ni de désordres par érosion, cavitation... lors de l'épisode de crues. Une érosion aval (enrochements de protection par exemple) peut être autorisée si les désordres occasionnés par le passage de la crue de dimensionnement restent limités et s'il est prévu des procédures de maintenance associées.

\footnotetext{
${ }^{7}$ Le dimensionnement de l'évacuateur de crues est donc un processus itératif dans la mesure où écrêtement et capacité d'évacuation des crues sont directement liés.
} 


\subsubsection{Analyse du risque « crues » pour les PPI}

Les Plans Particuliers d'Intervention (PPI) de barrages concernent a minima les grands barrages de hauteur au moins égale à $20 \mathrm{~m}$ et retenant un volume d'eau supérieur ou égal à 15 millions de $\mathrm{m}^{3}$. Ces plans sont établis à partir d'un dossier d'analyse des risques qui est transmis par le responsable de l'ouvrage et qui traite notamment des crues.

Dans la majorité des dossiers d'analyse des risques soumis à l'avis du CTPB depuis le décret du 15 septembre 1992, l'analyse du risque «crues » indique la cote de danger du barrage, définie par la cote de la retenue au-delà de laquelle l'exploitant estime ne plus pouvoir garantir la sécurité de l'ouvrage. II s'agit de la cote ultime qui, si elle était atteinte, aurait imposé à l'exploitant de passer à l'état de péril imminent (déclenchement de l'alerte et notamment des systèmes d'alerte aux populations). En théorie, elle est inférieure ou égale à la cote de stabilité ultime de l'ouvrage. En outre, l'exploitant peut considérer que la décision de passage en état ultime d'alerte peut être prise avant l'atteinte de cette cote si certaines situations (coupure de l'accès à certains organes essentiels, submersion de l'usine, ...) conduisent à une perte du contrôle du barrage.

Pour un ouvrage correctement dimensionné et sans pathologie particulière, la cote de danger ${ }^{8}$ est généralement fixée de façon forfaitaire et sans vérification particulière de la stabilité comme suit :

- barrages en matériau meuble (terre ou enrochement) :

$\circ$ barrages en terre homogène ou en enrochement avec masque amont : cote de la crête ou celle du sommet du masque, sans tenir compte d'un pare-vague éventuel,

- barrages zonés à noyau: cote du sommet du noyau ou une cote un peu supérieure si le remblai situé entre le sommet du noyau et la crête peut supporter une percolation temporaire ;

- barrages rigides (maçonnerie ou béton) : cote du couronnement ou, éventuellement, une cote supérieure.

Afin d'évaluer la sensibilité de l'ouvrage vis-à-vis du risque «crues », l'analyse des risques indique l'hydrogramme de crue, de forme générale comparable à celle de l'hydrogramme de la crue de projet, qui provoque la montée des eaux jusqu'à la cote de danger. Pour ce calcul, on tient compte du laminage de la retenue, et, le cas échéant, de la non ouverture de la vanne de l'évacuateur de plus forte débitance ; l'estimation de la sensibilité du barrage aux crues résulte d'une comparaison de cette crue à la crue de projet.

\subsubsection{Cas des petits barrages}

Pour les petits barrages en terre, le document «Petits Barrages » du CFGB (CFGB, 1997), d'un usage très courant et faisant actuellement référence dans la profession, propose (tableau 1 en page 25 du document) une modulation de la période de retour de la crue exceptionnelle, avec une valeur minimale de 1000 ans si l'ouvrage intéresse la sécurité publique. Cette modulation dépend d'un critère de risque intrinsèque représenté par un paramètre numérique égal à $\mathrm{H}^{2} \mathrm{~V}^{0,5}$ et dont la définition est identique à celle du décret du 11 décembre 2007. Cette formule combine la hauteur $\mathrm{H}$ (en $\mathrm{m}$ ) du barrage au-dessus du terrain naturel et le volume $\mathrm{V}$ (en millions $\mathrm{de}^{3}$ ) de la retenue à la cote de retenue normale.

\footnotetext{
${ }^{8}$ L'attention est attirée sur le fait que la majorité des dossiers PPI fixe la cote de danger de façon forfaitaire ; dans certains cas, cette cote est différente de la cote définie au 4.3.1.2.
} 


\begin{tabular}{|c|c|c|c|c|c|}
\hline $\mathrm{H}^{2} \mathrm{~V}^{0,5}$ & $<5$ & 5 à 30 & 30 à 100 & 100 à 700 & $>700$ \\
\hline $\begin{array}{c}\text { Périodes de retour en } \\
\text { années (crue) }\end{array}$ & 100 & 500 & 1000 & 5000 & 10000 \\
\hline
\end{tabular}

Tableau 4.1 - Périodes de retour des crues exceptionnelles pour les petits barrages en terre [CFGB, 2000]

\subsection{Réglementations et pratiques étrangères}

\subsubsection{Documentation}

Quelques réglementations et pratiques étrangères ont été examinées, en privilégiant les pays " occidentaux » ayant récemment conduit une réflexion sur la sécurité en crue des barrages. Le tableau 4.2 ci-dessous en dresse la liste :

\begin{tabular}{|c|c|c|c|}
\hline Pays & Texte de référence & Nature de ce texte & Date \\
\hline $\begin{array}{l}\text { Australie - } \\
\text { New South } \\
\text { Wales }\end{array}$ & $\begin{array}{l}\text { "Dam safety committee } \\
\text { guidelines" }\end{array}$ & $\begin{array}{l}\text { Recommandations } \\
\text { émises par l'autorité } \\
\text { de contrôle (Dams } \\
\text { Safety Committee) }\end{array}$ & 2002 \\
\hline Brésil & $\begin{array}{l}\text { «Criterios de Projeto Civil de } \\
\text { Usinas Hidrelectricas » }\end{array}$ & $\begin{array}{l}\text { Recommandations } \\
\text { émises par le Comité } \\
\text { Brésilien des } \\
\text { Barrages } \\
\end{array}$ & 2003 \\
\hline États-Unis & $\begin{array}{l}\text { «Federal Guidelines for Dam } \\
\text { Safety » }\end{array}$ & $\begin{array}{l}\text { Recommandations } \\
\text { (non obligatoires) } \\
\text { émises par l'agence } \\
\text { fédérale FEMA }\end{array}$ & 1998 \\
\hline Italie & $\begin{array}{l}\text { Loi de 1959, «règles techniques } \\
\text { » de } 1982 \\
\text { Demande émise par l'Autorité } \\
\text { Italienne des Barrages (RID) }\end{array}$ & \begin{tabular}{|l} 
Loi \\
Circulaire \\
(demandant ré- \\
examen sécurité en \\
crue des barrages).
\end{tabular} & $\begin{array}{l}1982 \\
2004\end{array}$ \\
\hline Norvège & $\begin{array}{l}\text { Norwegian Dam Safety } \\
\text { Regulation, Water Ressources } \\
\text { Act }\end{array}$ & Loi & 2010 \\
\hline Québec & Loi sur la sécurité des barrages & Loi & 2002 \\
\hline $\begin{array}{l}\text { Royaume } \\
\text { Uni }\end{array}$ & $\begin{array}{l}\text { Reservoir Act : projet de mise à } \\
\text { jour }\end{array}$ & Loi & $1975 ; 2011$ (projet) \\
\hline Suède & $\begin{array}{l}\text { "Swedish Guidelines for Design } \\
\text { Flood Determination for Dams" }\end{array}$ & $\begin{array}{l}\text { Recommandations } \\
\text { issues de la } \\
\text { profession (maîtres } \\
\text { d'ouvrages) }\end{array}$ & 2007 \\
\hline Suisse & $\begin{array}{l}\text { «Documentation de base } \\
\text { relative à la vérification de la } \\
\text { sécurité en } \\
\text { cas de crue ». }\end{array}$ & $\begin{array}{l}\text { Directive émise par } \\
\text { l'Office Fédéral }\end{array}$ & 2008 \\
\hline
\end{tabular}

Tableau 4.2 - Réglementations et pratiques étrangères examinées

Une fiche de synthèse par pays est fournie en annexe 1. 


\subsubsection{Classement des barrages}

Toutes ces références étrangères graduent les crues à considérer pour le dimensionnement selon des classes d'ouvrages. Ces classes dépendent des dimensions de la retenue et/ou des conséquences en cas de rupture.

\begin{tabular}{|l|l|}
\hline Pays & Modalités de classement des barrages \\
\hline $\begin{array}{l}\text { Australie - New } \\
\text { South Wales }\end{array}$ & $\begin{array}{l}\text { Croisement de deux critères : } \\
-\quad \text { Risque humain (5 classes) } \\
-\quad \text { Sévérité des dommages économiques }\end{array}$ \\
\hline Brésil & $\begin{array}{l}\text { Deux classes : critères géométriques mais prenant en compte } \\
\text { également le risque de perte de vies humaines (habitations } \\
\text { permanentes ou non à l'aval) }\end{array}$ \\
\hline États-Unis & Deux classes : selon qu'il y a risque humain ou non \\
\hline Italie & - \\
\hline Norvège & Selon risque humain aval en cinq classes \\
\hline Québec & $\begin{array}{l}\text { Croisement de deux critères : } \\
-\quad \text { Risque humain } \\
-\end{array}$ \\
\hline Royaume Uni & Selon rérité des dommage humain aval en trois classes \\
\hline Suède & Deux classes : selon qu'il y a risque humain ou non \\
\hline Suisse & - \\
\hline
\end{tabular}

Tableau 4.3 - Modalités de classement des barrages

La Suisse et l'Italie ont opté pour une classification des barrages basée sur la seule hauteur. Dans le cas de l'Italie, ce choix a été fait en 1982, et n'a pas été reconsidéré par la circulaire de 2004. Dans le cas de la Suisse, il s'agit d'un choix récent.

Les pays de culture anglo-saxonne, le Québec et les pays du Nord de l'Europe considérés dans ce recensement ont tous opté pour un classement basé sur le risque aval. $\mathrm{Ce}$ classement est parfois simplement dichotomique (avec ou sans risque), parfois plus fin. Dans l'estimation du risque aval, priorité est donnée à une estimation du risque incrémental causé par le barrage.

\subsubsection{Crues considérées}

Un ensemble de critères de dimensionnement des barrages considère simultanément : (1) la définition des crues à considérer, (2) l'état de disponibilité des évacuateurs, (3) la revanche éventuelle. ${ }^{9}$

\subsubsection{Crues pour les barrages de plus forte classe}

Le tableau 4.4 ci-dessous rassemble ces critères, exprimés ici pour le cas d'un barrage de plus forte classe. Ces critères ont été classés en "Crue de projet 》 ou "Crue de sécurité " selon qu'une revanche plus ou moins importante est considérée.

\footnotetext{
${ }^{9}$ Il faudrait également ajouter dans cette liste les méthodes d'évaluation de ces crues (plus ou moins prudentes). Mais les textes de référence ne donnent pas beaucoup d'indications à cet égard.
} 
Les critères de revanche indiqués dans ce tableau correspondent au cas d'un barrage en remblai.

Lorsque les calculs intègrent un critère de disponibilité des évacuateurs, ce critère est mentionné dans le tableau. Dans certains cas, la défaillance de l'évacuateur est traitée séparément, par une situation de projet spécifique ; ces cas sont mentionnés au tableau 4.4.

\begin{tabular}{|c|c|c|}
\hline Pays & Crue de projet & Crue de sécurité \\
\hline $\begin{array}{l}\text { Australie - } \\
\text { New } \\
\text { South } \\
\text { Wales }\end{array}$ & 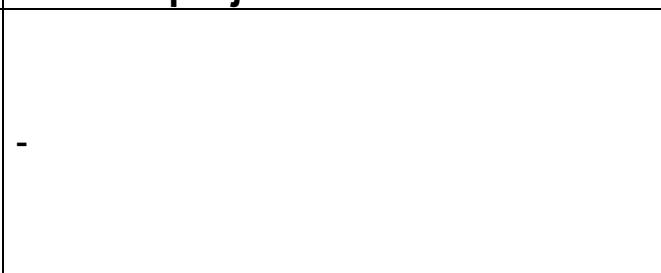 & $\begin{array}{l}\text { PMF (Probable Maximum Flood) } \\
\text { Disponibilité évacuateur : selon } \\
\text { analyse de fiabilité } \\
\text { Revanche }:=R\left[T ; \quad \text { I] } \quad{ }^{*}\right) \\
\text { Revanche mini }: 0,3 \text { à } 0,6 \mathrm{~m}\end{array}$ \\
\hline Brésil & PMF & - \\
\hline États-Unis & $\begin{array}{l}\text { PMF } \\
\text { Disponibilité évacuateur : selon } \\
\text { analyse de fiabilité } \\
\text { Revanche }=\mathrm{R}[\mathrm{T}, \mathrm{V}, \mathrm{S} . . .] \\
\end{array}$ & - \\
\hline Italie & $\begin{array}{l}T=1000 \text { ans } \\
\text { Revanche }=R[T, V, \ldots]+\text { sécurité. } \\
\text { Sécurité }: 1,5 \text { à } 4 \mathrm{~m} \text { pour les remblais } \\
\text { selon hauteur }\end{array}$ & - \\
\hline Norvège & $\begin{array}{l}\mathrm{T}=1000 \text { ans } \\
\text { Revanche }=\mathrm{R}[\mathrm{V}]+0,5 \mathrm{~m}\end{array}$ & \begin{tabular}{|l} 
PMF \\
Cote de danger
\end{tabular} \\
\hline Québec & 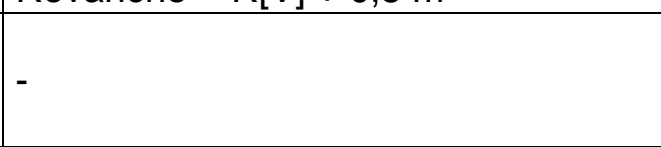 & $\begin{array}{l}\text { PMF } \\
\text { Revanche }=0\end{array}$ \\
\hline $\begin{array}{l}\text { Royaume } \\
\text { Uni }\end{array}$ & $\begin{array}{l}\text { PMF } \\
T=10000 \text { ans si la surverse est } \\
\text { tolérable } \\
\text { Revanche }=R[V] \text {, vague du vent max } \\
\text { annuel } 1 \mathrm{~h}\end{array}$ & 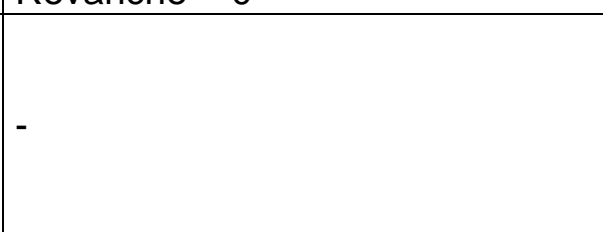 \\
\hline Suède & $\begin{array}{l}\text { Crue de projet : Pluie Max Régionale } \\
\text { avec ruissellement } 100 \% \text {. } \\
\text { Revanche = R[V], pour le vent } 20 \text { - } \\
25 \mathrm{~m} / \mathrm{s} \text {. }\end{array}$ & - \\
\hline Suisse & $\begin{array}{l}\text { Crue } T=1000 \text { ans, hydrogramme } \\
Q B(T) \\
\text { Revanche = } 1 \text { à } 3 \text { m pour remblais } \\
\text { selon hauteur } \\
\text { Calculs } 1 \text { vanne fermée }\end{array}$ & $\begin{array}{l}\text { Crue : Qs }(t)=1.5^{\star} Q_{\mathrm{B}}(2 / 3 \mathrm{t}) \\
\text { ou PMF } \\
\text { Cote de danger } \\
\text { Remblais : calculs } 1 \text { vanne fermée }\end{array}$ \\
\hline
\end{tabular}

$\left({ }^{*}\right)$ la revanche est calculée en tenant compte de différents aspects : V (vagues), T (tassements), S (séisme), I (incertitudes hydrologiques). Revanche $=\mathrm{R}[\mathrm{V}, \mathrm{T}, \ldots]$ signifie : " la revanche est déterminée en tenant compte des vagues, des tassements et d'éventuels autres aspects ».

\section{Tableau 4.4 - Crues de projet et de sécurité}

La PMF est presque toujours considérée. Mais elle ressort parfois d'une crue de projet (avec revanche significative au-dessus des Plus Hautes Eaux) et parfois d'une crue de sécurité (avec peu ou pas de revanche). 
Trois pays proposent une crue de projet nettement plus faible que la PMF ( $T=1000$ ans). Mais ils comblent au moins partiellement le déficit de sécurité par des critères très sévères de revanche (Italie) ou par une double niveau de vérification (Suisse, Norvège).

L'approche suédoise est difficile à comparer. Selon ses concepteurs, la crue calculée est de l'ordre de grandeur de $\mathrm{T}=10000$ ans.

\subsubsection{Note sur les barrages de classes inférieures}

À l'exception de Québec, le tableau 4.4 vaut pour tous les barrages qui posent un risque significatif. Ainsi, le tableau ci-dessus reste valable dans le cas d'un barrage "moyen » (typiquement, hauteur $20 \mathrm{~m}$, avec quelques dizaines d'habitations dans l'emprise de l'onde de rupture sous une hauteur d'eau d'au moins $1 \mathrm{~m}$ ).

La PMF est réservée aux barrages à très forts enjeux par le Québec (sinon : $T=1000$ à 10000 ans).

Pour les barrages à risque humain «négligeable », la crue centennale est régulièrement considérée.

\subsubsection{Note sur les parapets et la conception de la crête}

Plusieurs règlements recommandent de porter l'étanchéité le plus haut possible. La question des parapets, et de leur éventuelle contribution à la revanche, n'est pas abordée.

\subsubsection{Note sur les barrages en béton}

Les réglementations étrangères ne considèrent pas des crues différentes pour les barrages en béton et les barrages en remblai. Le cas des barrages en béton est traité par une revanche plus faible.

\subsubsection{Situations particulières de défaillance des ouvrages d'évacuation}

Plusieurs pays ajoutent une vérification de la sécurité du barrage en cas de défaillance des ouvrages d'évacuation:

\begin{tabular}{|l|l|}
\hline Pays & Situation « évacuateur défaillant » \\
\hline $\begin{array}{l}\text { Australie - New South } \\
\text { Wales }\end{array}$ & $\begin{array}{l}\text { Néant (l'éventuelle défaillance des évacuateurs est intégrée aux } \\
\text { calculs de référence) }\end{array}$ \\
\hline Brésil & Non spécifié \\
\hline États-Unis & $\begin{array}{l}\text { Néant (l'éventuelle défaillance des évacuateurs est intégrée aux } \\
\text { calculs de référence) }\end{array}$ \\
\hline Italie & $\begin{array}{l}\text { Évacuateurs vannés : réduction de } 50 \% \text { de la débitance } \\
\text { Flottants : si portée }<10 \mathrm{~m} \text { ou tirant d'air < } 1 \mathrm{~m} \text {, réduction de } 50 \% \\
\text { de la débitance }\end{array}$ \\
& $\begin{array}{l}\text { Critère : perte de seulement } 50 \% \text { de la « sécurité », et sécurité } \\
\text { résiduelle }>1 \mathrm{~m}\end{array}$ \\
\hline
\end{tabular}




\begin{tabular}{|l|l|}
\hline Pays & Situation « évacuateur défaillant » \\
\hline Norvège & $\begin{array}{l}\text { Vannes : 1 à 3 vannes coincées selon le nombre de vannes } \\
\text { Flottants : 25\% de perte de débitance si bassin très boisé. } \\
\text { Critère : Cote de danger }\end{array}$ \\
\hline Québec & Néant \\
\hline Royaume Uni & $\begin{array}{l}\text { Une vanne inopérante : la crue de 150 ans doit passer sous les } \\
\text { PHE. }\end{array}$ \\
\hline Suède & Non spécifié \\
\hline Suisse & $\begin{array}{l}\text { Néant (l'éventuelle défaillance des évacuateurs est intégrée aux } \\
\text { calculs de référence) }\end{array}$ \\
\hline
\end{tabular}

\section{Tableau 4.5 - Situations d'évacuateur défaillant}

À l'exception de Québec et de la Suède, le risque de défaillance des évacuateurs est considéré. Deux pratiques sont utilisées:

- des recommandations quantitatives (indépendantes de la nature des vannages et de leur commande) qui définissent le nombre de vannes inopérantes ;

- le recours à l'analyse de fiabilité.

La défaillance des évacuateurs est parfois intégrée aux calculs de référence, et parfois considérée comme une situation supplémentaire.

Italie et Norvège ont explicitement intégré le risque d'obstruction par les corps flottants, mais avec des approches (identification des situations à risques) et des critères très différents.

\subsection{Propositions pour le dimensionnement ou la vérification des évacuateurs de crues en fonction de la classe (vulnérabilité), du type de barrage et du type d'évacuateur}

Ce paragraphe propose des critères généraux de dimensionnement sans entrer dans le détail des calculs hydrauliques, ni revenir sur les dispositions constructives évoquées au chapitre 3. En particulier, le lecteur intéressé par les calculs de débitance des évacuateurs, des coursiers, des dissipateurs d'énergie... devra se référer aux publications spécialisées et bien connues du domaine (cf. annexe 4). Comme indiqué en introduction, les critères proposés s'inscrivent dans le contexte français, à la date de rédaction du présent rapport. Ils pourraient être différents pour d'autres pays.

\subsubsection{Cotes de référence}

\subsubsection{Cote des PHE}

Elle est définie au point 1.1.3 du chapitre 1. À cette cote, la stabilité du barrage est vérifiée avec des coefficients de sécurité correspondant à des cas de charge exceptionnels.

\subsubsection{Cote de danger}

Elle est définie au point 1.1.4 du chapitre 1. L'atteinte de cette cote constitue une situation extrême pour laquelle on examine des conditions d'états-limites ultimes pour l'ouvrage. 
La cote de danger tient compte des modes de rupture de l'ouvrage, de sa sécurité structurale et celle des appuis.

Sauf justifications particulières ou précautions motivées par l'état de l'ouvrage, les recommandations suivantes s'appliquent à la détermination de cette cote :

- pour un barrage en remblai semi-homogène ou à masque amont : cote de la crête ou du sommet du masque, sans tenir compte d'un éventuel parapet. On devra également se préoccuper de la résistance du parement aval au déferlement de vagues ;

- pour un barrage en remblai zoné : cote du sommet du noyau (ou une cote supérieure si on peut justifier que le remblai situé entre le sommet du noyau et la crête peut subir sans dégradation préjudiciable une percolation temporaire ou s'il est étanché par un écran) ;

- pour tous types de barrages en remblai, avec un coursier en contact avec le remblai : cote conduisant au débordement du coursier de l'évacuateur de crues, avec érosion du talus aval du remblai (en tenant compte, le cas échéant, de la formation d'ondes stationnaires et du gonflement de la lame d'eau par entraînement d'air) ;

- pour un barrage-poids, un barrage à contreforts ou un barrage-voûte avec des culéespoids : cote correspondant à l'atteinte d'un critère d'état-limite ultime de résistance de la structure ou de la fondation (cisaillement), avec les jeux de coefficients partiels s'appliquant à la catégorie des situations extrêmes. L'attention est attirée sur la sensibilité aux variations de cote des barrages-poids de hauteur modeste. Leur stabilité peut aussi être compromise par la sous-pression résultant d'un niveau d'eau aval important. Par ailleurs, la rupture d'un barrage-poids en maçonnerie peut se produire dans la masse de l'ouvrage avant déversement ;

- pour un barrage-voûte, dont les culées ne sont pas l'élément dimensionnant (sinon, cas précédent) : cote pouvant être sensiblement supérieure à la crête.

Pour les barrages pour lesquels la cote de danger est supérieure à celle de la crête, il convient de mener deux vérifications complémentaires qui pourront amener à modifier la cote initialement déterminée :

- vérification de la tenue ou non du parapet à la poussée hydrostatique et des corps flottants ;

- vérification de la résistance à l'érosion sur les appuis et en pied aval du barrage, en tenant compte des débits et de la durée de la surverse.

Le calcul de la cote de danger peut s'avérer délicat (par exemple lorsque la rupture du barrage est plus conditionnée par des questions d'érosion que par une résistance mécanique, qui peut être très élevée comme dans le cas des voûtes). Le résultat peut en outre être très sensible au choix de certains paramètres (comme une résistance à la traction). En tout état de cause, il conviendra de retenir une estimation prudente, après un calcul de sensibilité par rapport à la valeur des paramètres.

On attire également l'attention sur la présence éventuelle d'un parapet en crête : il convient de tenir compte de sa continuité, de sa résistance à la charge hydraulique, et de statuer sur l'aspect favorable ou défavorable de cette présence, suivant les cas. Par exemple, dans le cas d'un barrage en remblai, un parapet continu de rive à rive et résistant à la poussée de l'eau améliore la sécurité vis-à-vis des vagues et des crues, et peut permettre, sur justifications particulières, d'augmenter la cote de danger par rapport au même barrage sans parapet. Inversement, dans le cas d'un barrage-poids, un parapet continu et résistant peut être défavorable pour la situation extrême, la crue permettant d'atteindre la cote de danger pouvant alors être plus fréquente que sans parapet, suivant que la cote de danger est plutôt déterminée par des considérations de poussée hydrostatique ou par des considérations d'érosion due à la surverse. 


\subsubsection{Situations de projet}

\subsubsection{Généralités}

Les situations de projet modélisent un ensemble de conditions physiques représentant les conditions réelles auxquelles l'ouvrage est soumis et qui se produisent au cours d'une certaine durée pendant laquelle les distributions de toutes les données (actions, résistances) sont considérées comme constantes. Elles correspondent à des chargements que l'ouvrage est susceptible de connaître durant sa vie: cote normale d'exploitation, crue de projet, séisme, retenue vide, défaillance particulière d'un composant de l'ouvrage, etc.

Le concepteur définit les situations qu'il envisage pour le projet. Pour cela, il tient compte de la conception du projet, du mode de fonctionnement des installations, de l'environnement auquel l'ouvrage est soumis et de la vraisemblance des situations de défaillances.

Les conditions d'exploitation de l'ouvrage et les sollicitations hydrauliques associées permettent au concepteur de définir les situations de projet hydrauliques, qui revêtent une importance particulière pour les barrages.

Les situations de projet fixent les états-limites à justifier, selon l'ouvrage considéré. Les situations de projet à considérer doivent être sélectionnées en tenant compte des circonstances dans lesquelles l'ouvrage doit remplir sa fonction. Elles sont classées de la manière suivante :

- les situations normales d'exploitation. Elles se réfèrent aux conditions d'exploitation normale de l'ouvrage, et notamment hors crue ;

- les situations transitoires ou rares ${ }^{10}$. Elles se réfèrent à des conditions temporaires de fonctionnement ou à des probabilités d'occurrence assez élevées sur la durée de vie de l'ouvrage ;

- les situations exceptionnelles. Elles se réfèrent à des conditions exceptionnelles applicables à l'ouvrage ou à des probabilités d'occurrence faibles sur la durée de vie de l'ouvrage ;

- les situations accidentelles ou extrêmes. Elles se réfèrent à des conditions extrêmes applicables à l'ouvrage ou à des probabilités d'occurrence très faibles sur la durée de vie de l'ouvrage.

Dans ce document, nous nous intéressons plus particulièrement aux situations de crues pour tous les types de barrages.

\subsubsection{Situation normale d'exploitation}

Dans ce document, la situation normale d'exploitation n'est considérée que pour le dimensionnement de la revanche par rapport à la RN. Cette situation correspond à la cote atteinte dans la retenue pour la cote normale d'exploitation. Dans le cas particulier des barrages ayant un objectif principal d'écrêtement des crues, c'est le niveau du pertuis de fond ou le niveau correspondant au plan d'eau semi-permanent.

\footnotetext{
${ }^{10}$ On propose la nuance suivante entre transitoire et rare : une situation transitoire est une situation de courte durée (au regard de la durée de vie de l'ouvrage) dont la survenue est quasiment certaine pendant la vie de l'ouvrage (fin de construction, vidange), alors qu'une situation rare est une situation dont la probabilité d'occurrence est assez élevée (sans pour autant être égale ou proche de 1) pendant la vie de l'ouvrage.
} 


\subsubsection{Situation rare de crue}

Cette situation de projet concerne essentiellement les barrages écrêteurs de crues. Le projeteur peut, dans certains cas, être amené à considérer une situation rare de crue pour les barrages de stockage.

Pour un barrage écrêteur de crues normalement équipé d'un pertuis de fond et d'un déversoir de surface, on évalue le débit maximal non dommageable du cours d'eau en aval du barrage (par exemple débit de plein bord du lit mineur ou débit d'un ouvrage aval créant section de contrôle) et on dimensionne les pertuis pour délivrer au maximum ce débit. Lors des crues, il y a stockage temporaire dans la tranche d'eau de laminage et évacuation d'un débit plafonné par ces pertuis, jusqu'à atteindre la cote d'un déversoir de surface dont la fonction est d'assurer la sécurité du barrage lors des événements extrêmes. La situation rare de crue correspond donc à un niveau de remplissage atteignant la cote de protection, c'està-dire la cote du déversoir de surface.

La cote de protection est associée à une crue ou à une famille de crues qui, vu la présence de l'ouvrage, ne seront pas dommageables pour les enjeux en aval du barrage.

La probabilité annuelle de dépassement de la cote de protection associée à cette famille de crues est habituellement de $10^{-1}$ à $10^{-2}$ selon les enjeux à protéger, soit une période de retour de 10 à 100 ans. Le choix de la valeur de cette probabilité (ou période de retour) relève du maître d'ouvrage et répond à des considérations économiques et d'aménagement du territoire. A cette cote, la vérification de la stabilité structurelle de l'ouvrage est effectuée de la même façon et avec les mêmes coefficients de sécurité que ceux qui sont requis en situation normale d'exploitation.

\subsubsection{Situation exceptionnelle de crue}

Cette situation de projet concerne tous les barrages.

La situation exceptionnelle de crue conduisant à la cote des PHE peut résulter de divers événements hydrologiques, depuis la crue courte présentant un débit de pointe très élevé (donc écrêtée par la retenue) jusqu'à la crue longue présentant un débit durablement élevé (écrêtement réduit) en passant par les crues à pointes multiples (écrêtement de la première pointe, mais pas des suivantes).

La situation exceptionnelle de crue correspond à un niveau de remplissage laissant encore une revanche pour se protéger de l'effet des vagues et des irrégularités de la ligne d'eau, mais plus faible que pour la situation normale d'exploitation. Pour cette situation de crue, l'ouvrage doit répondre à tous les standards de sécurité, que ce soit sur le plan structural (résistance au cisaillement ou au glissement, résistance en fondation, résistance à l'érosion interne, résistance à l'affouillement) ou sur le plan hydraulique (marge par rapport au débordement des coursiers, ouvrages de dissipation correctement dimensionnés). On dispose donc encore de marges avant d'atteindre des états-limites de rupture.

La probabilité annuelle de dépassement de cette situation de projet est fixée au paragraphe 4.3.3.2.1 ci-après.

\subsubsection{Situation extrême de crue}

Cette situation de projet concerne tous les barrages. Elle est définie par l'atteinte de la cote de danger. 
Cet état peut résulter soit d'une crue extrême avec un fonctionnement nominal de l'évacuateur de crues, soit d'une crue plus faible combinée avec un dysfonctionnement de l'évacuateur de crues (cf. 4.3.3.2.2).

En effet, la capacité d'évacuation des crues peut être réduite par certaines défaillances d'éléments ou de composants impliqués directement dans la sécurité des évacuateurs, ce qui amène à des niveaux hydrauliques potentiellement plus défavorables.

Par ailleurs, pour les barrages en rivière, il convient également d'envisager deux situations extrêmes supplémentaires :

- combinaison séisme de base d'exploitation (SBE) et passe batardée durablement pour les barrages situés en zone 3 et 4 ;

- combinaison situation exceptionnelle de crue et passe batardée durablement.

\subsubsection{Autres situations de projet liées à une indisponibilité totale ou partielle de l'évacuateur de crues}

Certaines défaillances d'éléments ou de composants impliqués directement dans la sécurité de l'évacuateur de crues peuvent conduire à des situations rares, exceptionnelles ou extrêmes devant être spécifiquement étudiées. Parmi celles-ci, on peut citer (voir chapitre 3 pour plus de détails) :

- la défaillance d'une ou plusieurs vannes ;

- la défaillance d'un évacuateur de surface par obstruction partielle ou totale du fait d'embâcles.

La détermination des situations liées aux défaillances des éléments de sécurité de l'évacuateur relève d'études spécifiques d'analyse de risques, qui fixent au cas par cas les défaillances potentielles. Pour les barrages de classes A et B, les études d'analyse de risques sont intégrées dans les études de dangers.

Les études d'analyse de risques devraient permettre à terme d'estimer les probabilités de défaillance de l'élément particulier analysé combinée au niveau de crue. On évalue ainsi une probabilité d'occurrence globale rattachée à un scénario combinant simultanément la défaillance d'un composant et un niveau d'eau.

Le niveau de détail et la précision de l'étude d'analyse de risques sont adaptés à la taille et aux enjeux de l'ouvrage. Pour une première approche, on pourra se contenter d'une estimation des probabilités de défaillance à dire d'expert ou à partir de la littérature, et on limitera le nombre de situations examinées à quelques combinaisons " défaillances / niveau de crue » jugées a priori les plus caractéristiques ou les plus défavorables. Cette première approche permet de juger de l'opportunité d'études d'analyse de risques plus complètes.

En fonction de la probabilité ainsi évaluée, la situation examinée peut être considérée comme situation rare, exceptionnelle ou extrême.

Toutefois, la question de l'estimation des probabilités de défaillances des évacuateurs de crues doit être considérée comme du domaine de la recherche ; les études de dangers en cours pourront contribuer à faire progresser cette thématique. II est aujourd'hui quasiment impossible de fixer des probabilités de défaillance avec une précision suffisante pour que la démarche proposée ci-dessus relève réellement d'une utilisation opérationnelle. A minima, il est nécessaire d'effectuer une estimation prudente des probabilités de défaillances, de 
réaliser des études de sensibilité pour les probabilités estimées de défaillance. On peut aussi explorer des règles forfaitaires de type $\mathrm{N}-1$ (prise en compte de la possibilité de défaillance d'une passe de l'évacuateur de crue) et avec calcul de la crue conduisant à une cote de retenue exceptionnelle voire extrême.

En outre, il convient d'intégrer à la réflexion les conditions de maintenance périodique des ouvrages qui peuvent régulièrement rendre indisponibles certaines des passes d'un évacuateur, ce qui peut conduire à diminuer la sécurité en crue d'un barrage. Par exemple :

- batardage d'une ou plusieurs passes d'un évacuateur, aux fins de maintenance des vannes. Par exemple sur le Rhône, il est prévu tous les 40 à 50 ans une révision complète des vannes wagons des barrages mobiles. Démontage, réparation et remontage durent 6 mois. II y a 6 vannes à traiter. Sur 50 ans, le fait d'avoir 47 ans avec $N$ vannes $\left(Q_{1000}\right)$ et 3 ans avec $N-1$ vannes multiplie les risques par 2 environ sur la période. Ceci concerne les situations extrêmes mais aussi les situations exceptionnelles ;

- intervention sur la crête (ou l'étanchéité) du barrage, avec abaissement temporaire de la cote de crête (ou des PHE admissibles) ;

- intervention sur la structure du barrage, avec affaiblissement temporaire de sa résistance.

II y a donc lieu de définir les critères de sécurité à considérer pendant ces phases transitoires et éventuellement d'en tenir compte dans la conception.

L'approche privilégiée consiste à considérer que la sécurité en crue de l'aménagement ne doit pas être significativement diminuée pendant les travaux : les crues de référence restent celles de l'ouvrage en service. La sécurité du barrage est alors acquise par une adaptation des méthodes d'intervention (batardage), un éventuel abaissement de la cote de retenue pendant les travaux, et par le choix d'une saison d'intervention à moindre risque hydrologique.

Cette approche n'est pas toujours raisonnablement envisageable. Dans certains cas, il faut nécessairement admettre une sécurité moins forte pendant la période de travaux. La démarche consiste alors à évaluer quels sont les critères de sécurité " raisonnablement envisageables » pour la période des travaux. II faut pour cela démontrer que :

- les critères de sécurité retenus sont bien calés au mieux de ce qui est raisonnablement envisageable ;

- les gains escomptés de l'opération justifient bien les risques accrus pendant les travaux, ce qui peut par exemple être apprécié en comparant la probabilité de rupture en service normal et la probabilité de rupture pendant les travaux;

- des mesures appropriées sont prises pour gérer les situations de crise pendant les travaux ;

- sauf cas de barrages de classe $D$ et sauf cas très particulier, la sécurité pendant les travaux est acquise pour la crue centennale.

Nota: ces réserves sur les critères hydrologiques en conditions de maintenance ne s'appliquent pas au choix des critères hydrologiques pour la construction de nouveaux barrages.

\subsubsection{Dimensionnement hydrologique}

\subsubsection{1 Écrêtement des crues}


L'écrêtement d'une crue, tenant compte le cas échéant des champs d'expansion et/ou des barrages amont, est un comportement hydraulique dynamique de la crue dû au stockage provisoire d'une partie du volume de la crue dans une retenue. II se traduit par une déformation de l'hydrogramme de la crue : l'hydrogramme des débits sortants est différent de l'hydrogramme des débits entrants, parfois de manière importante ; il est également décalé temporellement.

Les conséquences de l'écrêtement sont généralement les suivantes:

- diminution du débit de pointe de crue à évacuer par rapport au débit de pointe entrant dans la retenue ;

- décalage dans le temps de la pointe de la crue ;

- décroissance retardée du débit pendant la décrue.

\subsubsection{1 Écrêtement par la retenue concernée}

Pour calculer l'effet du laminage par la retenue, on considère que le niveau de la retenue à l'arrivée de l'épisode de crue est égal à la cote de RN.

De facto, les barrages pour lesquels la cote des PHE est égale à celle de $\mathrm{RN}$ ne permettent pas la prise en compte de l'écrêtement en situation exceptionnelle de crue.

\subsubsection{2 Écrêtement par une retenue ou un champ d'expansion amont}

$\mathrm{Si}$, en amont d'un barrage, une retenue ou un champ d'expansion est susceptible d'écrêter naturellement le volume d'eau arrivant dans son bassin versant propre en l'absence de manœuvres volontaires d'exploitation (cas par exemple d'un barrage équipé d'un seuil déversant et où la cote de retenue normale est calée sous la cote du seuil), il faut en tenir compte car l'hydrogramme naturel de la crue arrivant au barrage aval sera déformé par l'effet de l'écrêtement amont, mécanique et inéluctable.

Si l'écrêtement éventuel résultait d'une exploitation particulière de l'ouvrage amont (par le biais d'une consigne de crue), il est recommandé de ne pas en tenir compte pour le dimensionnement de l'évacuateur de crues du barrage aval.

\subsubsection{Critères de dimensionnement}

Des critères de dimensionnement sont proposés en situation exceptionnelle et en situation extrême. Le respect de ces deux critères est préconisé pour tous les barrages. Pour les barrages existants, le groupe de travail recommande que soient respectés en priorité les critères en situation extrême; une vérification en situation exceptionnelle sera systématiquement réalisée et elle pourra, le cas échéant et en fonction de l'importance des enjeux et des risques, permettre d'apprécier le niveau de priorité d'une mise à niveau des capacités d'évacuation des crues ou de la fiabilisation de l'évacuateur de crues (en lien avec 4.3.2.6).

Par ailleurs et pour tenir compte de l'existant, il a été choisi de différencier, en situation exceptionnelle, le cas des barrages rigides (poids béton et maçonnerie, voûtes, contreforts, etc.) de celui des barrages meubles (terre, enrochements, etc.), afin de ne pas trop s'écarter des règles précédemment utilisées. Dans l'absolu, il eut été préférable de prendre en compte une période de retour indépendante du type de barrage (l'aléa hydrologique contre lequel on souhaite se protéger ne devrait a priori pas dépendre du type d'ouvrage). 


\subsection{En situation exceptionnelle}

Cette vérification, en continuité avec les pratiques antérieures, constitue une bonne ligne de défense en profondeur de la sûreté sans que, dans cette situation, la sécurité ultime des avalisants soit mise en cause. Les risques à ce stade concernent la dégradation potentielle limitée des installations. Le groupe de travail recommande que les vérifications en situation exceptionnelle soient menées avec les hypothèses suivantes :

- possibilité de laminage par la retenue et les ouvrages amont et pour une cote initiale de retenue correspondant à la cote maximale en situation normale d'exploitation (RN) ;

- prise en compte de la débitance des évacuateurs sans réduction pour cause de dysfonctionnement (sauf restrictions exposées en 3.2.2) ;

- $\quad$ prise en compte d'une crue de période de retour dépendant du type d'ouvrage et de sa classe (définie par le décret 2007-1735 du 11 décembre 2007) selon le tableau cidessous :

\begin{tabular}{|c|c|c|}
\hline & $\begin{array}{c}\text { Barrages } \\
\text { rigides }\end{array}$ & $\begin{array}{c}\text { Barrages } \\
\text { meubles }\end{array}$ \\
\hline$A$ & 1000 à 3000 & 10000 \\
\hline B & 1000 & 3000 \\
\hline$C$ & 300 & 1000 \\
\hline D avec V $\geq 50000 \mathrm{~m}^{3}$ & 100 & 300 \\
\hline
\end{tabular}

Tableau 4.6 - Périodes de retour des crues en situation exceptionnelle

La fourchette pour les barrages rigides de classe A résulte de la difficulté du groupe de travail à obtenir un consensus complet sur ce point. Une certaine latitude pourrait ainsi être donnée pour juger de la nécessité de recalibrer un évacuateur (la valeur basse de la fourchette) ou pour un dimensionnement nouveau faisant appel à la fourchette haute.

Pour les ouvrages mixtes comportant par exemple un bloc central en béton entouré de deux digues en remblai, on retient la crue la plus défavorable.

Pour les barrages non classés, il reste nécessaire de disposer d'organes d'évacuation des crues, si possible par seuil libre, ainsi que d'une revanche adéquate, voire d'un pare-vagues. II ne paraît pas raisonnable de dimensionner ces ouvrages pour une crue plus faible que la crue centennale.

Si la rupture d'un barrage amont peut entraîner la rupture d'un barrage aval, le barrage amont doit être dimensionné pour tenir compte de ce risque et donc éventuellement être surclassé.

\subsection{En situation extrême}

Les recommandations de cet alinéa s'appliquent aux ouvrages classiques. L'objectif de la vérification en situation extrême est d'assurer que, au-delà d'une situation déjà considérée comme exceptionnelle ou en cas de dysfonctionnement des organes d'évacuation des crues, le barrage, son système d'évacuation des crues, le cas échéant, les organes hydrauliques annexes (sous réserve qu'on démontre qu'ils restent opérationnels), voire même en tenant compte du débit déversé sur la crête, possèdent encore une marge de sécurité suffisante avant la survenance d'un accident. Celui-ci est supposé se produire à partir du moment où la retenue atteint, d'une façon ou d'une autre, la cote de danger. 
Avec les limites d'application indiquées en introduction au document, les probabilités cibles associées à ces événements sont les suivantes:

\begin{tabular}{|c|c|}
\hline Classe du barrage & Probabilité annuelle de dépassement \\
\hline $\mathrm{A}^{11}$ & $10^{-5}$ \\
\hline $\mathrm{B}$ & $3.10^{-5}$ \\
\hline $\mathrm{C}$ & $10^{-4}$ \\
\hline $\mathrm{D}$ avec $\mathrm{V} \geq 50000 \mathrm{~m}^{3}$ & $10^{-3}$ \\
\hline
\end{tabular}

Tableau 4.7 - Probabilités annuelles de dépassement en situation extrême

Dans certains cas, on peut penser que, à situation hydrologique donnée, l'impact supplémentaire à l'aval sur les personnes et les biens lié à la rupture du barrage est négligeable. Sous réserve que cette hypothèse soit démontrée, notamment à l'occasion d'une analyse de risques, cela peut permettre une augmentation de la probabilité cible pour cet ouvrage (cf. 5.6).

La probabilité annuelle de $10^{-5}$ correspond par exemple à une probabilité de $10^{-3}$ pour une durée de vie de 100 ans.

L'estimation des crues de probabilité de dépassement inférieure à $10^{-4}$ est considérée comme très hasardeuse. On recommande à ce stade d'estimer la crue de période de retour 100000 ans en majorant la crue décamillennale par un coefficient multiplicateur sur les débits de l'ordre de 1,3 et d'estimer la crue de période de retour 33000 ans en la majorant par un coefficient multiplicateur sur les débits de l'ordre de 1,15 . On se reportera à la section 5.4 (méthodes en développement) pour le cas des bassins avec une forte capacité d'interception dont le comportement en situation extrême reste très difficile à modéliser.

Les vérifications sont menées en prenant en compte le laminage par la retenue et les ouvrages amont et pour une cote initiale de retenue correspondant à la cote maximale en situation normale d'exploitation (RN).

Les vérifications sont menées avec deux jeux d'hypothèses :

1/ Avec un fonctionnement nominal de l'évacuateur de crues: la débitance des organes hydrauliques est la débitance théorique à la cote de danger, sans réduction pour cause de dysfonctionnement.

2/ Avec un dysfonctionnement de l'évacuateur de crues.

Étude spécifique de la fiabilité de l'évacuateur de crue:

La crue à prendre en compte est telle que la survenance de cette crue combinée avec le dysfonctionnement de l'évacuateur de crues a une probabilité globale correspondant au tableau 4.7 ci-avant. L'attention est attirée sur le lien possible entre le niveau de crue et les possibilités de défaillance de l'évacuateur de crues.

Il peut y avoir plusieurs vérifications avec plusieurs niveaux de dysfonctionnement ou d'obstruction de l'évacuateur.

\footnotetext{
${ }^{11}$ La classe A regroupe notamment tous les barrages dont la hauteur dépasse 20 mètres au-dessus du terrain naturel et donc avec des potentiels de dangerosité très différents. Pour les plus importants d'entre eux, une diminution de la probabilité cible n'est pas à écarter
} 
Cette approche est a priori celle qui est préconisée lorsqu'une telle analyse paraît possible, en particulier pour les barrages soumis à étude de dangers (classes A et B). Cependant, la plus grande attention est à apporter à la justification des paramètres utilisés pour une telle étude (taux de défaillance possible, en lien avec les procédures de maintenance, d'essais, etc., lien entre ce taux et le niveau de crue, etc.).

\section{Pas d'étude spécifique de la fiabilité de l'évacuateur de crues:}

On vérifie que la cote de la retenue ne dépasse pas la cote de danger avec une crue dont la période de retour est celle de la crue exceptionnelle mais avec un évacuateur amputé de la passe de plus grande débitance et sous réserve de la vérification d'absence de mode commun de non fonctionnement simultané de plusieurs passes.

\section{Nota :}

Pour les évacuateurs vannés, il est nécessaire de considérer la possibilité de blocage total de l'ensemble des vannes en position fermée, situation qui pourrait générer des risques incrémentaux majorés. Pour les ouvrages des classes $C$ et $D$ munis de tels évacuateurs, non soumis à études de dangers et ne bénéficiant pas d'une étude particulière de fiabilité, on peut ainsi envisager le passage de crues de faible période de retour (annuelle voire décennale) toutes vannes bloquées en position fermée.

\subsubsection{Cas des retenues à barrages multiples}

L'évacuateur de crues concerne l'ensemble des barrages qui peuvent fermer une même retenue mais avec des hauteurs, des volumes d'eau relâchés en cas de rupture différents et donc des classes qui peuvent être différentes. Les critères de dimensionnement sont ceux associés au barrage de la classe la plus élevée. Au-delà de ces critères, il est prudent de privilégier la tenue du barrage pour lequel la vulnérabilité aval est la plus élevée (en général, la tenue du barrage principal par rapport à la tenue d'un ou des barrages secondaires). Les cotes de la crête de ces barrages peuvent être différenciées, afin de minimiser les risques résiduels.

Par exemple, dans le cas d'une retenue constituée d'un barrage principal en terre et d'un ou plusieurs barrages annexes en terre également, il est évidemment très intéressant que le dernier barrage qui risque de casser par submersion soit a priori le barrage principal (cf. fig. 4.1 ci-dessous).

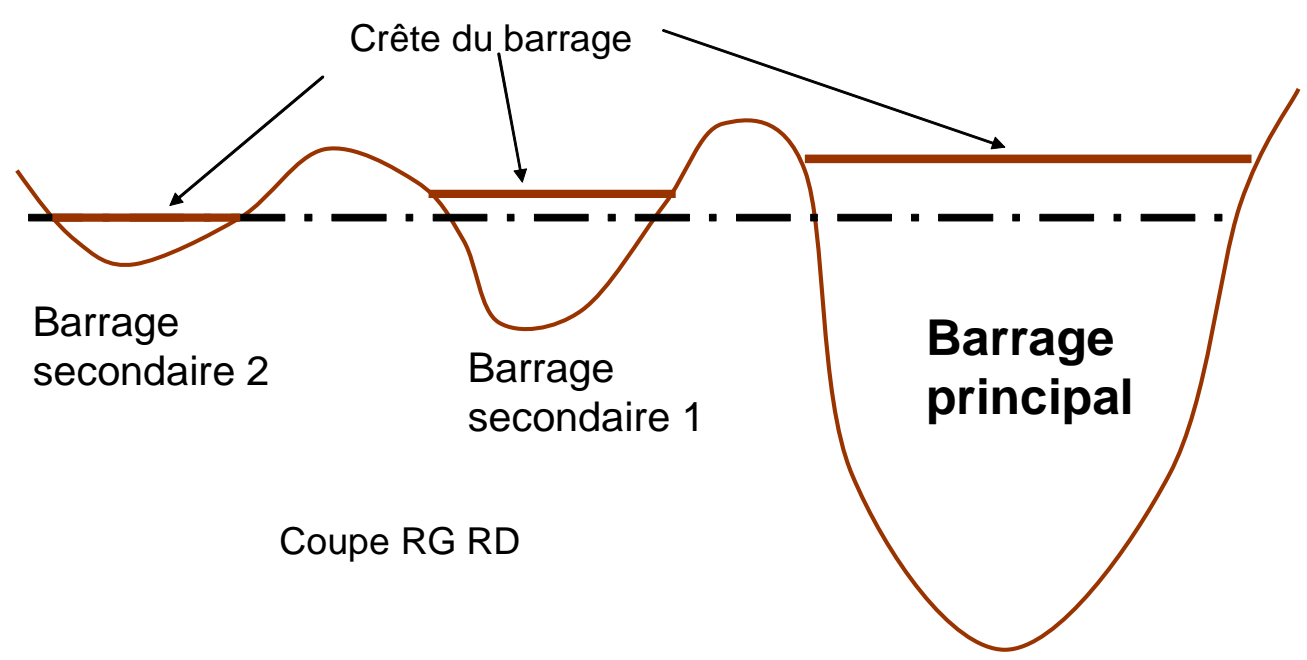

Figure 4.1 - Exemple d'une retenue constituée par 3 barrages en terre 
Dans ce cas, les barrages secondaires jouent un rôle de protection supplémentaire du barrage principal même bien au-delà de la crue extrême. On notera qu'un simple cavalier, d'un coût négligeable, suffit à orienter la rupture vers le bon ouvrage.

En cas de combinaison de barrages en terre, barrages-poids, barrages-voûtes, on peut par un raisonnement similaire réduire notablement les risques (cf. fig. 4.2 ci-dessous).

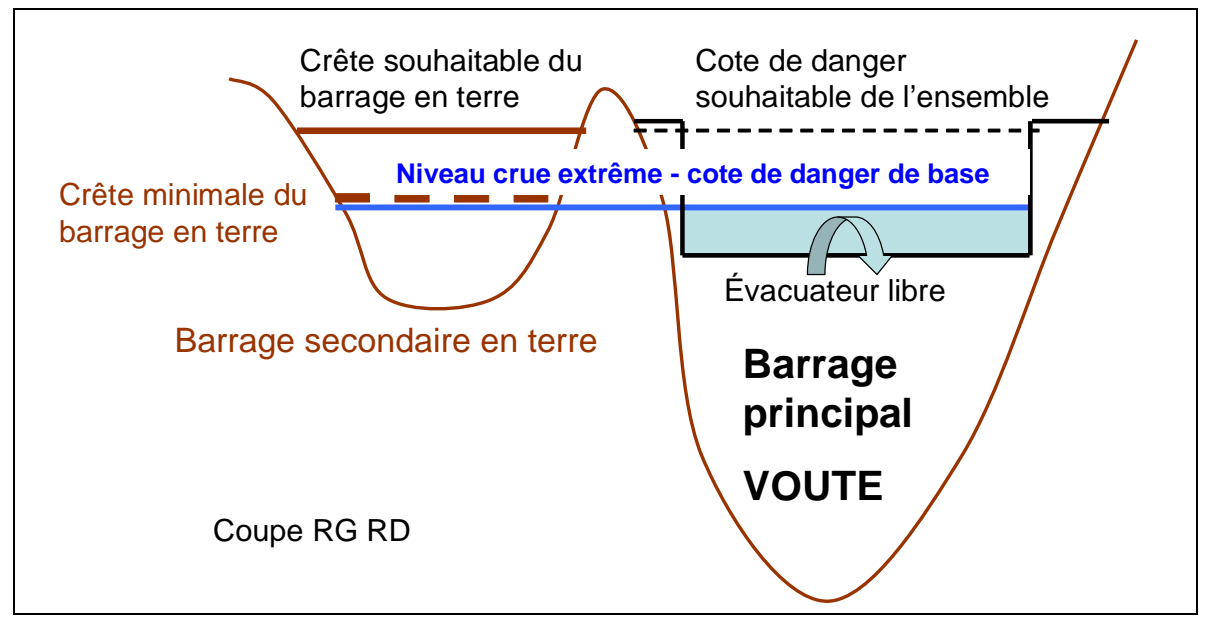

Figure 4.2 - Exemple d'une retenue constituée par un barrage-voûte et un barrage en terre

La cote minimale du barrage en terre est, selon les recommandations, calée par rapport au niveau maximum atteint par la crue extrême, cette cote correspond à la cote de danger de l'ensemble de l'aménagement. Or, il se trouve que l'ouvrage principal (barrage-voûte) admet une cote de danger supérieure (qui permettrait de passer un débit très supérieur à la crue extrême sans dommage). Dans ce cas, on obtiendra une diminution significative du risque de rupture du barrage en terre, en calant la cote de celui-ci plus haut, ne serait-ce qu'avec un simple cavalier. La sur-hauteur du barrage en terre peut être estimée en comparant coûts supplémentaires et risque évité.

\subsubsection{Cas des barrages écrêteurs de crue}

Un barrage écrêteur de crue comporte une retenue partiellement ou totalement vide (barrage sec) qui pourra stocker temporairement une partie du volume des crues, réduisant ainsi les débits aval donc les dégâts aval. On peut résumer l'effet du barrage en trois points :

- tant que la crue ne remplit pas la retenue au-delà de la cote de protection (voir définition en 1.1.2), il n'y a pas de débordements en aval (sous réserve d'un dimensionnement correct des pertuis) ; c'est ce qui se passe pour les crues courantes jusqu'à des périodes de retour de 50 à 100 ans selon l'objectif de protection assigné par le maître d'ouvrage ;

- pour les crues plus rares et jusqu'à la crue extrême qui remplit la retenue jusqu'à la cote de danger, le barrage continue à jouer un rôle d'écrêtement qui en général diminue avec la rareté de l'événement. Vu que l'évacuateur de surface entre en service (en plus des pertuis), le barrage ne permet plus d'éviter les débordements en aval, même si ces derniers sont moins importants que sans l'ouvrage. Toutefois, au début du fonctionnement de l'évacuateur de surface, le gradient de débit aval peut être plus fort que le gradient de la crue naturelle. Dans cette gamme de crues, le barrage continue à apporter un bénéfice, pour autant que l'on ait un dispositif d'alerte efficace ${ }^{12}$ et que le

\footnotetext{
${ }^{12}$ le dispositif d'alerte doit impérativement prendre en compte et donc anticiper le début de fonctionnement de l'évacuateur de surface, qui va se traduire par une augmentation rapide du débit relâché en aval.
} 
fonctionnement courant (qui gomme les crues en aval) n'ait pas conduit à oublier le risque associé aux fortes crues, voire à augmenter les enjeux dans le lit majeur sensément protégé ;

- pour des crues extrêmes amenant à dépasser la cote de danger, le barrage a une probabilité significative de se rompre. Les dégâts seront alors considérables et brutaux, le système d'alerte étant potentiellement moins efficace dans une telle situation (au moins en aval immédiat).

Une analyse de risque simplifiée permet de vérifier le bénéfice du barrage. Si le barrage a été correctement dimensionné :

- le risque inondation est pratiquement nul jusqu'à la crue de protection ;

- il est partiellement réduit dans le domaine intermédiaire jusqu'à la crue extrême ;

- il devient considérable au-delà.

Un calcul associant le chiffrage des dégâts évités et la probabilité de l'événement associé pour toute la gamme des périodes de retour jusqu'à la crue de danger permet, par intégration, d'estimer le bénéfice moyen annuel apporté par le barrage, soit B.

Cependant, le barrage construit fait courir chaque année à l'aval le risque de rupture dont le calcul économique est le suivant :

$$
\mathrm{R}=\mathrm{PV} \text {, }
$$

avec $\mathrm{P}: \quad$ probabilité de la crue extrême atteignant la cote de danger ;

$V$ : vulnérabilité, montant des dégâts pour cette crue.

Pour justifier l'investissement I de la construction du barrage (que l'on amortit en général sur $\mathrm{n}=30$ à 50 ans), il est nécessaire de vérifier que $\mathrm{B}$ est nettement supérieur à $R+\mathrm{l} / \mathrm{n}$. Et ce d'autant plus que le calcul ne porte que sur les aspects économiques, sans inclure les risques pour les personnes, beaucoup plus difficiles à estimer.

Si B est proche de $R+I / n$, le barrage ne fait que remplacer un risque par un autre, ce qui ne justifie pas son financement.

Cette approche est recommandée pour des projets neufs mais peut aussi être déployée lorsqu'un barrage existant nécessite d'importants investissements de confortement ou de remise à niveau.

\subsubsection{Détermination de la revanche}

Comme indiqué au 1.1.5, la revanche est la différence de cote entre la retenue et la crête du barrage. Cette revanche est dimensionnée à partir de critères sur la hauteur des vagues, tant par rapport à la $\mathrm{RN}$ que par rapport aux PHE (cf. fig. 4.3). Elle peut, dans certains cas, inclure un parapet s'il est continu et joue le rôle de pare-vagues (cf. fig. 4.4). Elle n'est en aucun cas associée à la notion de sur-hauteur mise en place, sous forme de contre-flèche, sur les barrages meubles ou sur fondation compressible pour faire face aux futurs tassements de l'ouvrage. 


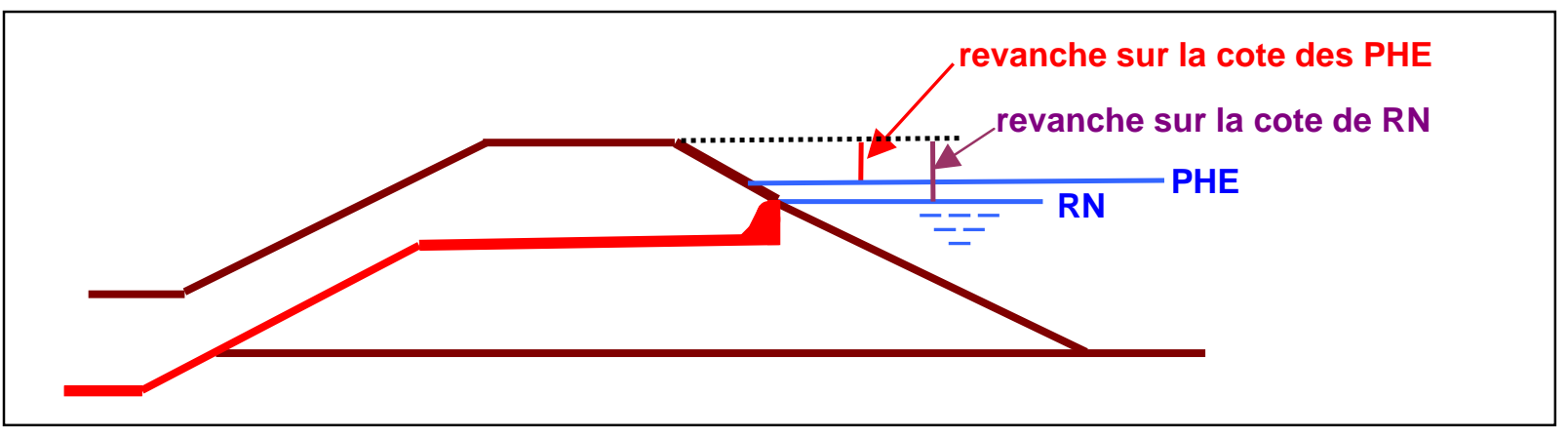

Figure 4.3 - Revanche sur les PHE ou sur la RN pour un barrage non équipé d'un mur pare-vagues

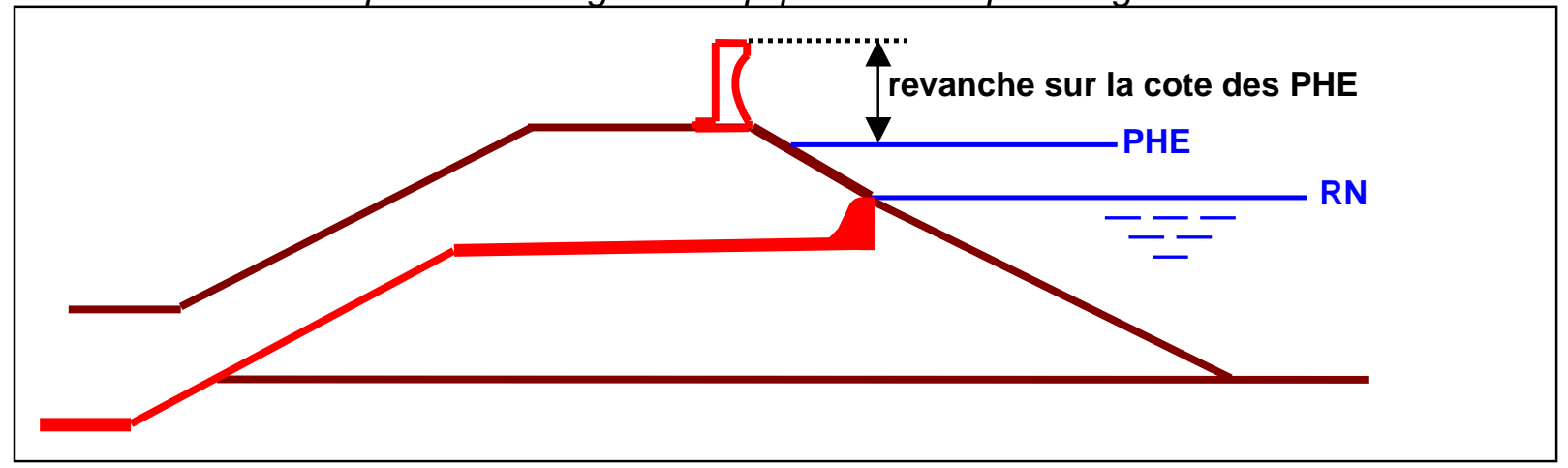

Figure 4.4 - Revanche sur les PHE pour un barrage équipé d'un mur pare-vagues

Le déroulement du calcul de la revanche est détaillé en annexe 2. Les étapes sont les suivantes.

\subsection{Détermination des caractéristiques du vent}

On détermine les caractéristiques du vent en considérant deux situations de projet :

- un vent de période de retour 50 ans soufflant sur une retenue qui se trouve à la cote des PHE ; c'est cette situation qui s'avère généralement dimensionnante, sauf pour les retenues où $\mathrm{RN}=\mathrm{PHE}$;

- un vent de période de retour 1000 ans sur la retenue normale.

\subsection{Estimation de la hauteur des vagues}

On s'intéresse à leur hauteur significative (notée $H_{s}$ ), c'est-à-dire la hauteur moyenne du tiers supérieur de toutes les vagues du spectre. Elle dépend de la vitesse du vent, de la longueur du fetch et de la profondeur de la retenue.

\subsection{Calcul de la vague de projet}

Notée $H_{d}$, elle dépend du pourcentage de vagues du spectre que l'on accepte de laisser dépasser la crête.

$$
H_{d}=K H_{s}
$$

On propose de retenir les valeurs suivantes pour le coefficient $K$ en fonction du type de barrage (plus particulièrement de son talus aval) : 


\begin{tabular}{|l|c|}
\hline \multicolumn{1}{|c|}{ Type de barrage } & $\boldsymbol{K}$ \\
\hline Béton ou maçonnerie & 0,75 \\
\hline Enrochements & 1,0 \\
\hline Terre & 1,25 \\
\hline
\end{tabular}

Tableau 4.8 - Coefficient $K$ en fonction du type de barrage

\subsection{Calcul de la hauteur de déferlement}

Pour la vague de projet, on estime la hauteur de déferlement (run-up en anglais), notée $R$, qui est la différence de hauteur verticale entre le niveau du plan d'eau et le niveau maximal atteint par le déferlement de la vague sur le parement amont. Cette hauteur dépend de la nature du barrage (la pente du parement, la rugosité du matériau) - voir tableaux en annexe 2.

4.3.3.5.5 Détermination de la cote de la crête du barrage ou du pare-vagues

À l'issue du calcul de hauteur de déferlement pour les deux situations de projet, on trouve la plus forte des cotes $R N+R_{R N}$ et PHE $+R_{P H E}$ et on fixe la cote de la crête (après tassement) au moins à cette valeur ; en variante, on peut atteindre cette cote à l'aide d'un mur parevagues construit contre le bord amont de la crête. Dans ce cas, la cote de crête doit être au moins égale à celle des PHE. II est recommandé que la hauteur du pare-vagues ne dépasse pas $1,5 \mathrm{~m}$.

\subsection{Conclusion}

La revanche nécessaire pour les vagues varie dans une large gamme en fonction des conditions de vent, de la longueur de la retenue, de la pente du talus amont et de sa rugosité. Des règles forfaitaires simplistes ne peuvent être suffisantes et un calcul est indispensable à chaque fois. Le cas d'un parement lisse de barrage en remblai est nettement le plus défavorable.

\subsubsection{Dimensionnement hydraulique}

\subsubsection{Principes généraux}

Les différentes situations de projet évoquées au paragraphe 4.3.2 impliquent le transit de certains débits par les organes d'évacuation. La justification hydraulique vise à s'assurer de la capacité des organes d'évacuation à évacuer ces débits pour les cotes déterminées à l'amont et à l'aval, avec le niveau de sécurité requis pour ces différentes situations de projets.

On s'assurera que ces ouvrages sont capables non seulement d'évacuer le débit souhaité (dimensionnement des pertuis d'évacuation) mais aussi de le transférer en aval sans risque pour l'ouvrage (par exemple, suivant le cas, suffisamment loin du barrage par des coursiers ou un saut de ski) et enfin de dissiper l'énergie au niveau de la restitution en aval du barrage (bassins ou fosses de dissipation).

À cause des marges de sécurité requises qui sont différentes d'une situation à l'autre, la situation la plus critique peut être suivant les cas une situation exceptionnelle ou une situation extrême, ou même dans certains cas une situation intermédiaire (comme le 
passage d'un écoulement à l'air libre dans une galerie à un écoulement en charge pour de plus forts débits, ou des situations intermédiaires plus critiques dans le cas d'un bassin de dissipation). La justification hydraulique étudiera donc l'ensemble des situations potentiellement critiques.

Le dimensionnement hydraulique doit viser à vérifier que le niveau de sécurité hydraulique des ouvrages est cohérent avec les niveaux de sécurité requis pour les autres aspects de la conception des ouvrages dans les différentes situations de projet.

Pour atteindre cet objectif, on serait tenté d'utiliser la notion de coefficient de sécurité, par exemple des coefficients du type rapport entre les forces stabilisatrices et les forces motrices comme il en existe en mécanique des structures. Cependant, on se heurte à plusieurs difficultés :

- certains aspects de la justification hydraulique échappent à la notion de coefficient de sécurité tel que défini ci-dessus; par exemple, c'est le cas pour l'apparition de la cavitation ou pour la limite de débordement sur les bajoyers d'un coursier d'évacuateur ;

- dans d'autres cas, un coefficient de sécurité tel que défini ci-dessus a physiquement du sens mais les formules de dimensionnement disponibles, souvent empiriques, n'indiquent pas le degré de sécurité déjà pris par les auteurs dans les dimensionnements proposés.

Ainsi, la justification hydraulique fait appel dans bien des cas au jugement et à l'expérience du concepteur, mais en tout cas on se fixera les objectifs suivants :

- les situations d'écoulement intermédiaires, normales et exceptionnelles ne doivent entraîner aucun dommage aux ouvrages, que ce soit les ouvrages d'évacuation euxmêmes ou les autres ouvrages potentiellement affectés par les écoulements ;

- les situations extrêmes peuvent entraîner des dommages aux ouvrages, à condition que ces dommages ne mettent pas en cause la fonctionnalité de l'évacuateur de crues et ne risquent pas d'entraîner par voie de conséquence la rupture du barrage (par exemple érosion au pied d'un barrage en enrochement suite à la destruction d'un coursier d'évacuateur de crues ou sous l'action de courants de retour causés par une dissipation d'énergie devenue insuffisante).

Les ouvrages hydrauliques présentant des situations non traitées par les formules ou abaques de dimensionnement classiques, et plus généralement les ouvrages devant transiter des débits importants ou pouvant présenter un risque pour la sécurité du barrage, doivent être étudiés à l'aide d'une modélisation spécifique, modélisation physique ou modélisation numérique si les modèles numériques disponibles sont capables de traiter correctement tous les aspects intervenant dans la formation des écoulements et la sécurité des ouvrages.

L'étude sur modèle devra étudier toutes les situations potentiellement critiques jusqu'aux situations extrêmes et même au-delà, ce qui permettra de fournir une information sur les marges de sécurité offertes par le dimensionnement.

Il est à noter que la modélisation physique est un outil précieux pour orienter et soutenir le jugement du concepteur par la possibilité offerte de représenter facilement diverses situations de fonctionnement, de visualiser les écoulements, de mesurer des vitesses d'écoulement, de représenter le cas échéant des lits érodables, et d'une manière générale par la possibilité offerte de tester facilement diverses modifications des ouvrages pouvant améliorer la sécurité à moindre coût. 
En cas d'augmentation du débit de projet lors d'un recalibrage des évacuateurs existants, il faut évaluer l'incidence de ce sur-débit sur les ouvrages de transfert des écoulements et de dissipation d'énergie.

\subsubsection{Débitance des ouvrages}

La détermination de la débitance d'un évacuateur de crues ressort d'un calcul hydraulique parfois complexe. Outre la géométrie réelle de l'ouvrage, qui conditionne sa capacité tant par ses dimensions que par son profil hydraulique, les conditions particulières d'écoulement en crue, notamment l'influence des évacuateurs voisins et celle de la ligne d'eau en aval, sont autant de facteurs à prendre en compte pour déterminer la débitance de l'ouvrage.

Par ailleurs, des conditions particulières éventuelles de fonctionnement et d'aération de lame d'eau peuvent conduire à des calculs complexes.

Il pourra être nécessaire, compte tenu de la complexité de certains ouvrages et de leur caractère unique, de recourir à un modèle hydraulique pour valider les calculs, tant numérique que physique.

II n'est pas d'usage d'appliquer des coefficients de sécurité à la débitance calculée ou mesurée sur un modèle réduit. En effet, une débitance sous-estimée conduirait au fait que des débits plus importants que prévu transiteraient dans l'ouvrage et cela pourrait causer des problèmes tels que le débordement des coursiers ou une énergie à dissiper plus importante que prévu (Lencastre, 1996).

\subsubsection{Coursiers des évacuateurs}

Ces coursiers, destinés à canaliser l'eau à l'aval des pertuis ou des seuils, ne sont à prévoir que si des dégâts sont à craindre sur le barrage (érosion, éboulements, cavitation, etc.) par le passage de la lame d'eau, et que de ce fait il faut éloigner du barrage la zone de restitution et de dissipation d'énergie des ouvrages d'évacuation.

Dans le cas des barrages en remblais, il est particulièrement important de donner une revanche suffisante aux bajoyers des coursiers quand ils sont implantés à proximité du barrage, afin de tenir compte de la formation éventuelle d'ondes stationnaires et des ressauts éventuels liés à l'usure des coursiers, aux décalages éventuels des joints et à l'aération de la lame d'eau.

Cette revanche doit être calculée en considérant une augmentation maximum de rugosité du coursier, ainsi qu'un décalage défavorable d'un joint de construction transversal à l'écoulement ou d'un obstacle équivalent. Elle doit tenir compte de l'entraînement d'air dans l'écoulement.

La formation d'ondes stationnaires est liée à la présence de singularités dans la section du coursier (angles vifs, rétrécissements brusques, etc.). Ces ondes, dont la localisation reste fixe pendant l'écoulement à un débit donné mais peut aussi se déplacer au fur et à mesure de la variation du débit, se réfléchissent lorsqu'elles rencontrent un des bajoyers et se développent ainsi vers l'aval. Leur hauteur peut largement dépasser celle de la lame d'eau. Les débordements éventuels vont donc se manifester toujours aux mêmes endroits pour un certain débit, ce qui peut conduire à de dangereuses érosions localisées. Le modèle réduit hydraulique avec des essais à différents débits reste le meilleur moyen pour mettre en évidence de tels phénomènes, même si les progrès récents de la modélisation numérique laissent entrevoir des développements prometteurs. 
Si tous les phénomènes indiqués ci-dessus ont bien été pris en compte dans le calcul, et ceci pour toutes les situations jusqu'aux situations extrêmes, on ne prendra pas de marge de sécurité supplémentaire par rapport au calcul en situation extrême. En général, un coursier ainsi dimensionné assurera une certaine marge de sécurité vis-à-vis du débordement pour les situations intermédiaires.

\subsubsection{Dissipation d'énergie}

La dissipation de l'énergie hydraulique lors de la chute de l'eau en aval des pertuis d'évacuateurs (à l'air libre ou en charge) peut créer, si elle n'est pas maîtrisée, de graves dégâts au point de restitution en rivière, pouvant aller jusqu'à la ruine des ouvrages.

Les chutes de faible et moyenne hauteur ne permettent pas d'éloigner suffisamment du barrage des jets libres issus des organes évacuateurs. Ces jets libres pourraient alors entraîner des érosions des fondations ou du pied du barrage. Si l'on ne peut pas dissiper l'énergie de la crue en coulant sur le rocher (matériau friable ou peu résistant aux fortes vitesses), une solution classique est de conduire l'eau provenant de l'organe évacuateur dans un coursier jusqu'au niveau de la restitution et de dissiper son énergie dans un bassin de dissipation à ressaut. Celui-ci peut être naturel, ou équipé de blocs disperseurs de formes diverses. De tels bassins ont été construits jusqu'à des chutes d'une centaine de mètres, mais leur champ d'application est plutôt les chutes de quelques dizaines de mètres. Les blocs disperseurs sont sensibles à l'érosion en cas de transport solide et sont aussi sujets à la cavitation au-dessus de certaines hauteurs de chute.

Lorsque la chute est plus importante, il est possible de projeter le jet suffisamment loin du barrage en utilisant un coursier terminé par une cuiller ou déflecteur, on parle alors de saut de ski. Les cuillers ou déflecteurs éventuellement munis de dents peuvent servir à étaler le jet à la restitution afin de diminuer l'énergie à dissiper par unité de surface, à augmenter la dissipation d'énergie dans l'air, ou encore à orienter les jets pour éloigner la restitution d'une zone sensible telle que le pied d'une rive ne devant pas être déstabilisée.

À la réception des jets, on devra toujours s'assurer que la dissipation d'énergie à la restitution est suffisante pour empêcher que le développement d'une fosse d'érosion ou l'action de tout courant direct ou de retour puisse éroder en pied et déstabiliser soit le barrage proprement dit, soit sa fondation, soit les rives en aval. Ce principe impose, dans la plupart des cas, de s'assurer de la présence d'un volume d'eau dans une fosse d'amortissement pour dissiper l'énergie à la réception du jet. La fosse d'amortissement peut être bétonnée ou ménagée sans revêtement dans le lit de la rivière, avec éventuellement des injections des joints du massif rocheux. Le niveau dans le bassin est contrôlé par la loi hauteur-débit de la rivière, ou surélevé par un contre-barrage aménagé à l'aval de la fosse de dissipation. On peut également pré-excaver la fosse de dissipation pour augmenter la profondeur d'eau.

Pour l'étude de ces dispositifs, et notamment les tapis de réception et fosses d'amortissement, les études sur modèle réduit sont indispensables pour préciser les formes et dimensions des ouvrages, en particulier les déflecteurs, et pour s'assurer qu'aucun écoulement à forte vitesse ne risque de mettre en péril les ouvrages, ou leurs fondations ou encore la stabilité des rives.

Le bassin à ressaut constitue un cas où la notion de coefficient de sécurité au sens de la mécanique (rapport des forces stabilisatrices sur les forces motrices) s'applique. En effet, le ressaut hydraulique reste fixé dans un bassin de dissipation tant que l'impulsion de l'écoulement en sortie du bassin, contrôlé par le chenal en aval si celui-ci est en régime fluvial, est plus grande que l'impulsion de l'écoulement amont issu de l'organe d'évacuation (on appelle impulsion la somme des forces de pression hydrostatique et de la quantité de 
mouvement). La profondeur d'eau aval dite « conjuguée » de la profondeur de l'écoulement torrentiel imposé par l'amont correspond à un coefficient de sécurité 1, c'est-à-dire à la limite du ressaut chassé. On calera le bassin de manière à ce que, dans les situations normales ou exceptionnelles, la profondeur d'eau aval soit au moins $10 \%$ plus grande que la profondeur d'eau conjuguée, ce qui revient à s'assurer que le coefficient de sécurité ou rapport des forces stabilisatrices (impulsion aval) sur les forces motrices (impulsion amont) vaut 1,2. Pour les situations extrêmes, on se contentera de vérifier que le coefficient de sécurité est supérieur ou égal à 1 (limite du ressaut chassé).

\subsubsection{Cavitation}

Tout au long du chemin d'eau au travers des ouvrages d'évacuation des crues, une attention particulière doit être portée sur le risque de cavitation entre la lame d'eau et les parements. En effet, des vitesses importantes de l'eau peuvent créer des dépressions au niveau des parements à la limite de l'écoulement soit du fait de l'existence de formes favorisant ces dépressions (seuil déprimé, rainures de vannes, dents, décrochements en général, etc.), soit du fait de l'état de surface de ces parements (coursier béton même neuf, ou pire encore éventuellement dégradé par du transport solide). Cette cavitation peut rapidement engendrer des dégradations et à l'extrême la ruine de l'ouvrage. L'étude théorique et sur modèle de l'écoulement sur le coursier doit explicitement analyser ce risque.

Des dispositifs tels que blindages ou aérateurs seront utilisés si nécessaire pour éviter des dommages par la cavitation.

Du point de vue des marges de sécurité, on retiendra que les ouvrages d'évacuation ne doivent subir aucun dégât du fait de la cavitation pour toutes les situations jusqu'aux situations exceptionnelles. La cavitation est acceptable pour les situations extrêmes, dans la mesure où dans le contexte de la France ces situations sont d'une durée relativement limitée.

\subsubsection{Cas des protections en enrochement}

Les zones de terrains érodables situées soit au voisinage de l'approche des pertuis d'évacuation, soit au voisinage des ouvrages de dissipation, doivent dans certains cas être protégées par des enrochements pour éviter que des érosions ne viennent déstabiliser les ouvrages construits.

La notion de coefficient de sécurité au sens de la mécanique (rapport des forces stabilisatrices sur les forces motrices) peut s'appliquer au cas des enrochements de protection. En effet, la résistance à l'arrachement d'un enrochement de protection par l'écoulement est liée à la valeur du coefficient d'arrachement adimensionnel (noté $\square^{*}$ ou $\mathrm{Q}$ ou $A=\square / \square g D$ ) introduit par Shields, qui caractérise le rapport entre le couple moteur tendant à renverser le bloc, représenté ici par la force tractrice de l'écoulement, et le couple stabilisateur représenté ici par la densité déjaugée $\mathrm{u}$ et le diamètre $\mathrm{D}$ (Ulrich, 1987).

Malheureusement, les formules courantes de dimensionnement des protections en enrochement telles que la formule d'lsbash $V=m(2 g a D)^{0,5}$ reliant le diamètre $D$ des enrochements de densité déjaugée $\square$ à la vitesse moyenne $V$ de l'écoulement avec un paramètre $m$ choisi suivant les circonstances, ne font apparaître clairement ni la limite d'arrachement ni le coefficient de sécurité.

Cependant, on peut déjà établir que : 
- Shields a conclu de ses essais de transport solide que le début d'entraînement des grains (assimilable à un coefficient de sécurité égal à 1) correspondait à un coefficient d'arrachement adimensionnel de l'ordre de 0,05, ce qui permet un dimensionnement lorsque le coefficient d'arrachement peut être calculé (cas d'un écoulement à couche limite développée dans un chenal protégé en enrochements) ;

- si on dispose d'un modèle réduit, on peut trouver avec ce modèle quelle situation d'écoulement correspond à la sécurité de 1 pour un diamètre d'enrochement testé, c'està-dire au départ des premiers blocs ;

- on augmente de $10 \%$ le coefficient de sécurité à l'arrachement ou rapport des forces stabilisatrices sur les forces motrices lorsqu'on augmente de $10 \%$ le diamètre des enrochements.

Enfin, une protection en enrochement n'a pas un diamètre unique mais est constituée par un fuseau granulométrique ou gravimétrique. Une sécurité suffisante doit être prise entre le diamètre $D$ fourni par les formules de dimensionnement et le fuseau de la protection. Par exemple, dans le cas des protections des berges ou du plafond d'un chenal, on assimile parfois le diamètre de dimensionnement $D$ issu des formules ou abaques au diamètre D25 du fuseau de la protection.

\subsubsection{Cotes atteintes par le remous de la crue}

Lors de crues importantes et selon la configuration de la retenue, le plan d'eau n'est plus horizontal et la cote en queue de retenue ou en un autre point éloigné du barrage est plus élevée que la cote du plan d'eau au contact du parement du barrage.

II est souvent nécessaire de calculer la ligne de remous en crue avec la cote PHE au barrage, afin de déterminer, en particulier en cas d'étude de rehausse de cette cote, la cote de submersion amont ainsi que l'incidence de cette ligne d'eau sur l'écoulement de la rivière en amont de la retenue (le remous dû au barrage peut remonter très loin au-delà de la queue de retenue, dans des zones aménagées après construction de l'ouvrage).

\subsubsection{Dimensionnement structurel et mécanique}

Les barrages dans leur conception et leur gestion relèvent de différents domaines. C'est le cas notamment pour les organes d'évacuation des crues : ouvrages de génie civil, ils doivent respecter les règles constructives en vigueur qu'il s'agisse des superstructures en béton, des vannes métalliques... Ils sont également équipés d'éléments mécaniques dont la manipulation et l'entretien doivent garantir la sécurité de l'ouvrage et de l'exploitant.

À défaut d'un cadre réglementaire spécifique, le dimensionnement structurel et mécanique peut faire appel à des textes généraux (en particulier dans le domaine du génie civil) ou à des documents issus d'autres domaines (cf. annexe 4).

II est recommandé de faire appel à une approche semi-probabiliste des dimensionnement/vérification des ouvrages. Ceci est cohérent avec :

- nature exceptionnelle des événements pris en compte, par exemple prise en compte de crues de grandes périodes de retour et éventuelle combinaison de plusieurs phénomènes jugés exceptionnels ;

- des incertitudes inhérentes à l'estimation des charges unitaires ;

- des incertitudes sur les caractéristiques de résistances des matériaux ;

- la prise en compte d'objectifs complémentaires vis-à-vis de l'apparition de désordres ou de la résistance ultime... 
À ce jour, les règlements ne couvrent pas tous les besoins du concepteur d'évacuateurs de crues, qui est laissé à son propre jugement sur de nombreux aspects, notamment la formulation des conditions naturelles et les calculs hydrauliques.

Le dimensionnement des équipements mécaniques, incluant la détermination des contraintes et des déplacements induits pour différentes combinaisons de charges et d'efforts de manœuvre, est souvent un exercice délicat mais qui doit être mené avec le plus grand soin.

Les aspects relatifs à la déformabilité des vannes et leurs conséquences sur l'étanchéité ne doivent pas être négligés.

\subsubsection{Cas de charge}

Des précautions doivent être prises en ce qui concerne la définition des cas de charges que les normes ne précisent pas. En effet, si les normes définissent les pondérations à appliquer en fonction du type de charges (charges permanentes, d'exploitation ou accidentelles), le choix des scénarios à considérer et de l'affectation du type de charges reste à définir par le concepteur en accord avec le maître d'ouvrage.

Les cas de fonctionnement en mode dégradé doivent être considérés pour le dimensionnement.

Exemple de charges appliquées (liste non exhaustive)

\begin{tabular}{|l|l|l|}
\hline Description de la charge & Caractéristique & Nature de la charge \\
\hline Poids propre & Poids propre de la structure & Permanente \\
\hline Retenue Normale amont & Pression hydrostatique & Permanente \\
\hline Retenue Normale aval & Pression hydrostatique & Permanente \\
\hline $\begin{array}{l}\text { Niveau PHE, Crue de Projet } \\
\text { Danger de Danger, Crue de } \\
\text { Danger }\end{array}$ & Pression hydrostatique & Variable \\
\hline Force de frottement, guidage & Force linéaire & Accidentelle \\
\hline $\begin{array}{l}\text { Chocs de bateaux ou corps } \\
\text { flottants }\end{array}$ & Force ponctuelle & Permanente \\
\hline Séisme & $\begin{array}{l}\text { Accélérations appliquées à la } \\
\text { masse des ouvrages, } \\
\text { surpressions } \\
\text { hydrodynamiques sur la face } \\
\text { amont des ouvrages et des } \\
\text { vannes }\end{array}$ & Accidentelle \\
\hline $\begin{array}{l}\text { Gel pris en compte } \\
\text { uniquement si aucune } \\
\text { disposition constructive n'est } \\
\text { prise pour l'éviter - chauffage } \\
\text { par exemple) }\end{array}$ & Poussée sur le tablier & Accidentelle \\
\hline Sédiments & $\begin{array}{l}\text { Capacité de blocage d'une } \\
\text { vanne }\end{array}$ & Permanente \\
\hline
\end{tabular}

$\left(^{*}\right)$ Dans le cas du choc d'un bateau ou de corps flottants, la charge est considérée comme accidentelle (non pondérée) dans le cas où il n'existe pas de dispositif de protection et où l'on souhaite que la structure résiste aux chocs des bateaux et/ou des corps flottants. 


\subsubsection{Vérification des déplacements}

Les déplacements des structures métalliques sont vérifiés avec la combinaison de type ELS.

Les déplacements limites peuvent être définis de la façon suivante, si $L$ est la largeur de la vanne :

- pour les vannes de surface, vannes tournantes ou levantes et clapets manœuvrés par deux vérins : la flèche admissible doit être inférieure à $L / 1000$;

- pour les clapets manœuvrés d'un seul côté, la déformation maximale de l'arête supérieure doit être inférieure à L/500.

Par ailleurs, les déplacements limites devront tenir compte des capacités d'adaptation en déformation des joints d'étanchéité.

\subsubsection{Cas particulier des barrages mobiles en rivière}

\subsubsection{Description générale}

« Un barrage mobile est un ouvrage qui comporte des éléments mobiles permettant de régler le niveau de la retenue lorsque le débit varie et pouvant être totalement effacés du cours d'eau, en période de crues, afin d'éviter que l'ouvrage ne provoque, par le remous qu'il crée, une surélévation du niveau atteint par les crues en amont » (Ginocchio, 1978).

Le volume de la retenue est très faible par rapport au volume des crues et les effets de laminage quasi inexistants.

À pleine ouverture des passes de l'évacuateur de crues, l'influence du barrage sur les niveaux d'eau atteints en amont est faible ; ces niveaux ne dépendent que du débit de la crue et non plus du dimensionnement des passes (aux pertes de charges provoquées par les piles du barrage près et sous réserve qu'aucun écoulement en charge ne se produise au droit de l'ouvrage, par la mise en charge des vannes levées ou d'un ouvrage supérieur de franchissement par exemple). Dans l'idéal, la rivière en crue devrait se comporter comme s'il n'y avait pas de barrage. Comme pour les barrages classiques, la connaissance des niveaux atteints au droit de l'ouvrage lors des crues imposent des dispositions de conception (élévation des passerelles, des motorisations, etc.).

Par ailleurs, les lignes d'eau à l'amont du barrage ne peuvent généralement plus être considérées comme horizontales lors du passage d'une crue et donc la sécurité peut également être conditionnée par une critère de non-dépassement de la ligne d'eau en un point situé bien à l'amont de l'ouvrage. La notion de cote de PHE et de cote de danger est donc plus complexe dans la mesure où elle signifie une cote au droit au barrage (et donc sa stabilité) mais elle implique aussi une ligne d'eau dans la rivière.

Lors des crues importantes, le cours d'eau retrouve son lit majeur. Les barrages en rivière sont donc souvent contournés par les flots, parfois très à l'amont. L'étude des crues (exceptionnelle et extrême) vis-à-vis de ce type d'ouvrage doit permettre d'apprécier son influence sur l'écoulement de l'eau et d'évaluer les débits contournant éventuellement l'ouvrage et qui ne transitent pas par le barrage lui-même.

Les calculs hydrauliques sont plus complexes que pour un barrage classique à retenue et nécessitent des modélisations fines, parfois en 3D, le cas échéant avec la prise en compte de la dynamique des phénomènes si les champs d'inondation amont sont importants. 
L'ouvrage pouvant être submergé lors des fortes crues, les calculs prennent en compte les cotes d'eau à l'aval du barrage.

Certains barrages de faible hauteur, ainsi que les petits barrages mobiles, peuvent être submergés par l'aval lors d'une crue majeure. Dans ce cas, l'incidence du passage de la crue s'apprécie par un calcul hydraulique mené de l'aval vers l'amont.

Enfin, la rupture de ces ouvrages en crue libère un volume d'eau généralement faible par rapport à celui de la crue et donc ne génère souvent qu'une surélévation limitée dans les zones déjà impactées à l'aval par l'inondation naturelle.

Sur les grands fleuves (par exemple, le Rhin ou le Rhône), les aménagements hydroélectriques peuvent être constitués de la manière suivante :

- un barrage situé sur le lit mineur de la rivière et constitué d'une série de vannes. Il est destiné à créer une chute ;

- une usine hydroélectrique et/ou une écluse, soit accolée(s) au barrage, soit en général en dérivation conformément à la figure 4.5 ci-après ;

- des digues latérales à l'amont du barrage et le long du canal d'amenée à l'usine. Cette disposition permet d'augmenter la chute et de ne pas empiéter sur les zones latérales agricoles ou parfois urbaines.

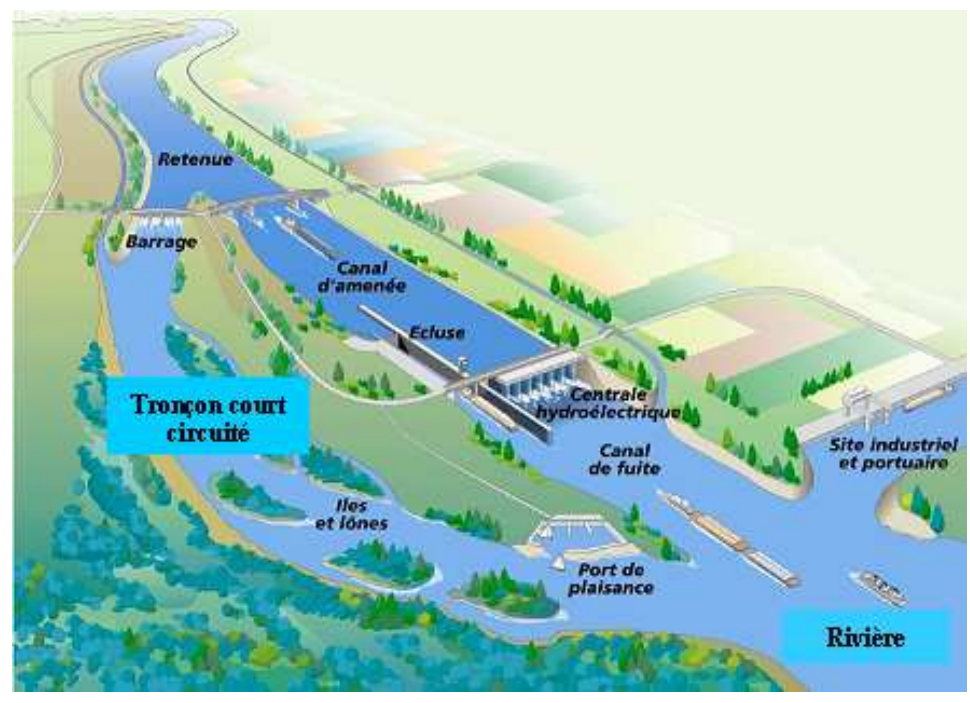

Figure 4.5 - Schéma type d'aménagement au fil de l'eau, en dérivation

En présence d'endiguement amont, il est primordial d'examiner la question du passage des crues sur l'ensemble de l'aménagement et pas seulement sur le barrage en rivière lui-même. Les digues sont conçues pour être insubmersibles pour les débits inférieurs ou égaux à celui de la crue exceptionnelle (et aussi sous l'effet des ondes de disjonction qui se produisent en cas d'arrêt brusque de la centrale hydroélectrique et qui se propagent à l'amont de l'usine en remontant le canal d'amenée puis la retenue et dont l'effet peut être amplifié par des odes secondaires, dites ondes de Favre).

Sur ces ouvrages, on prête une attention particulière au transit des sédiments dont les dépôts peuvent modifier le fond de la rivière et donc les niveaux atteints en crue. Cependant, pour les plus fortes crues, compte tenu des vitesses dans la retenue, il se peut que celle-ci soit auto-curante, ce qui doit être vérifié. Si ce n'est pas le cas, des dragages d'entretien doivent être effectués pour maintenir la débitance en crue. 


\subsubsection{Recommandations pour les barrages mobiles en rivières}

Il est proposé :

- de considérer l'aménagement dans son ensemble (voire même plus à l'amont), contournement des digues ou des appui compris; dans certains cas, il pourra être intéressant de favoriser ces contournements (ou de les localiser) grâce à des zones déversantes ;

- de dimensionner l'ensemble, et notamment les digues amont, pour des crues de fréquence définies comme les barrages classiques (cf. 4.3.3).

Par exemple, pour un aménagement en dérivation, on peut concevoir le schéma hydraulique suivant :

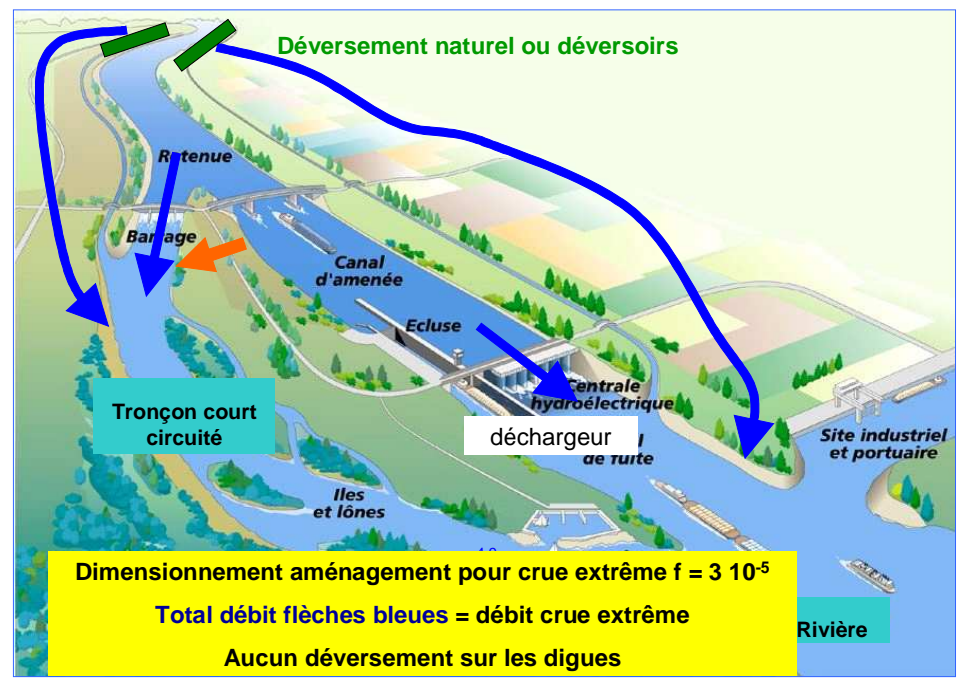

Figure 4.6 - Dimensionnement global proposé pour la crue extrême

Le débit de la crue extrême est assuré par (cf. fig. 4.6) :

- le débit du barrage toutes vannes ouvertes sous la cote de danger ;

- le débit de contournement de l'aménagement par l'amont, par déversement naturel sur les berges ou par des déversoirs appropriés si nécessaire ;

- accessoirement le débit de l'usine en déchargeur.

Le contournement par l'amont se fait en principe de manière naturelle, sinon il faut l'aider avec des déversoirs, digues fusibles, voire siphons positionnés aux endroits appropriés. De la même façon et pour les autres sections de digues, il peut être intéressant de privilégier, du fait de l'absence d'enjeux, des zones de débordement/rupture (flèche orange sur la figure 4.6 ci-dessus).

L'attention est attirée sur les conséquences du dimensionnement des ouvrages en cas de crues extrêmes. Ceci pourrait imposer :

- de sur-dimensionner la retenue: augmentation de la hauteur des digues et/ou de la largeur de la retenue ;

- de prolonger les endiguements vers l'amont, voire d'endiguer aussi les affluents (cf. fig. 4.7). 


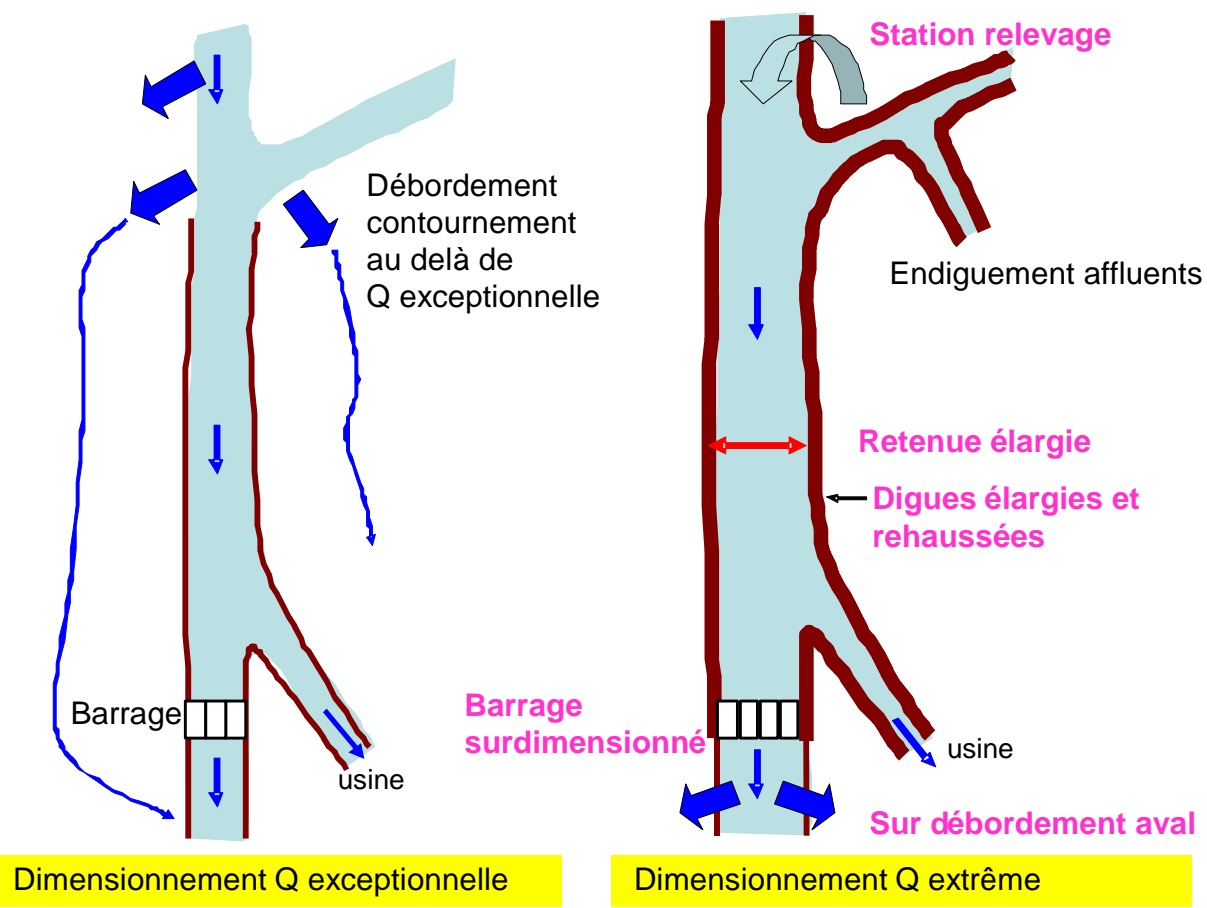

Figure 4.7 - Dimensionnement des digues pour la crue exceptionnelle ou pour la crue extrême, vue en plan

Les inconvénients peuvent être les suivants :

- suppression des zones naturelles d'expansion de crue, pouvant accroître les inondations à l'aval, soumis alors à une crue extrême non écrêtée ;

- sur-dimensionnement de la retenue, pouvant avoir des effets néfastes sur la morphologie :

- pour les petites crues, la sédimentation va être favorisée du fait de la diminution de vitesse induite par l'augmentation de section,

- pour les très fortes crues, le confinement d'un débit extrême avec des hauteurs d'eau anormalement hautes pourra au contraire déclencher une incision du lit dans la retenue ;

- création de coins d'eau entre rivière principale et affluent, nécessitant une station de relevage (cf. fig. 4.7).

\subsection{Adaptation des barrages existants aux nouvelles recommandations}

Les recommandations du présent document modifient les usages actuels, d'une part en fixant des périodes de retour de la crue amenant la retenue à PHE qui peuvent être différentes de celles utilisées à la construction (indépendamment d'une réévaluation éventuelle des crues), d'autre part en introduisant la notion de cote de danger et de crue extrême. Ceci est susceptible de conduire au constat d'un sous-dimensionnement d'un évacuateur de crues existant.

Un changement du mode d'exploitation de la retenue (en permettant par exemple une augmentation de la tranche de laminage) ou une modification de la cote des $\mathrm{PHE}^{13}$ peut

\footnotetext{
${ }^{13}$ Sous réserve d'une vérification par le calcul de la résistance suffisante de l'ouvrage et sans préjuger des problèmes d'autorisation administrative que ceci pourrait nécessiter.
} 
permettre de répondre simplement aux recommandations des paragraphes précédents. Dans le deuxième cas, il convient de vérifier l'ensemble de la chaîne hydraulique avec une nouvelle valeur de PHE (hauteur des bajoyers, tirants d'air sous une vanne, un pont, une passerelle, etc.).

Le cas échéant, des gains de débitance peuvent être obtenus par un reprofilage d'un seuil, sous réserve d'étudier les conséquences sur l'ensemble de la chaîne hydraulique. Si cela s'avérait insuffisant, des adaptations plus importantes de l'évacuateur peuvent être envisagées. L'annexe 3 fournit quelques exemples.

Enfin, il peut être nécessaire de recourir à la construction d'un seuil supplémentaire. Dans la majorité des cas, on doit privilégier une solution avec un seuil libre qui présente une meilleure garantie de robustesse par rapport à la variabilité possible des valeurs de crues. Ceci n'exclut pas d'intégrer à la réflexion des problématiques parallèles, comme par exemple le transit sédimentaire, qui peuvent plaider pour des solutions plus lourdes.

On rappelle en outre les règles de priorisation proposées au paragraphe 4.3.3.2.

\subsection{Utilisation des recommandations en dehors du contexte français}

Les présentes recommandations ont été préparées dans le contexte des caractéristiques du parc de barrages français et en relative continuité avec les pratiques en usage en France. Plusieurs points ne peuvent pas être transposés sans réflexion et doivent être adaptés pour être pertinents dans un autre contexte.

\subsubsection{Période de retour de la crue extrême}

Concernant les grands barrages, barrages de classe A dans la réglementation française, on peut noter que certaines réglementations internationales peuvent apparaître plus sévères que celles proposées dans les présentes recommandations.

La pratique française traditionnelle est fondée sur la notion de crue de projet avec une période de retour comprise entre 1000 ans et 10000 ans. L'adoption d'une vérification à la cote de danger correspondant à la crue extrême ou crue de danger (check flood) est une évolution majeure visant à vérifier le comportement ultime de l'ouvrage, en ligne avec l'évolution des pratiques internationales.

La période de retour proposée pour les crues extrêmes pour les barrages de classe A est équivalente à 100000 ans, alors que plusieurs réglementations étrangères s'appuient sur la PMF, crue réputée la plus forte possible. Compte tenu de la difficulté d'estimer la PMF, plusieurs réglementations étrangères proposent de retenir pour la crue extrême 1,5 fois la crue décamillennale. Les présentes recommandations proposent de retenir 1,3 fois la crue décamillennale (barrage de classe A), en fonction des connaissances actuelles sur les crues extrêmes. II faut insister ici sur le fait que l'estimation de ce ratio reste encore très approximative et qu'il n'y a pas de raison particulière à ce qu'il soit le même pour toutes les régions du monde.

À noter enfin que la France dispose de services de contrôle des ouvrages hydrauliques et que, à l'appui de ces services, le Comité technique permanent des barrages et des ouvrages hydrauliques émet un avis sur certains projets de nouveaux barrages ou de révision de 
barrages anciens. Les barrages moyens (classe B) et les grands barrages (classe A) font l'objet d'une étude de dangers périodique, fondée sur une analyse des risques, permettant une approche approfondie de la sûreté en crue dans ses diverses composantes (incertitude hydrologique, fiabilité des évacuateurs vannés, risque d'embâcles, etc.).

Ces spécificités françaises justifient les choix retenus dans les recommandations, qui ne sont pas directement transposables dans un autre contexte.

\subsubsection{Très petits barrages}

Les recommandations excluent les barrages non classés au sens de la réglementation française et les barrages de classe $D$ de moins de $50000 \mathrm{~m}^{3}$ de capacité.

Cette limite, fixée dans le contexte français, est à définir au cas par cas dans un autre contexte géographique et socio-économique. Le risque correspondant à ces petites retenues est en général très faible et il convient de ne pas décourager des solutions innovantes et économiques, en particulier les ouvrages déversants ou des évacuateurs de crues rustiques, et de ne pas empêcher un développement extrêmement utile et socialement indispensable de ces tout petits barrages dans certaines régions du monde. 


\section{Axes de recherches et de développements}

Le groupe de travail a mis en exergue quelques points évoqués dans les paragraphes suivants et sur lesquels il est indispensable de poursuivre la réflexion. Certains relèvent d'axes de recherche ; d'autres peuvent avoir un caractère opérationnel dans certains pays mais n'ont pas encore été mis en œuvre dans le contexte français.

Le groupe considère également que la thématique de l'acceptabilité du risque associé au comportement des barrages à l'occasion des crues majeures est une question primordiale mais qu'elle relève d'une démarche sociétale, voire politique, hors du champ du groupe de travail du CFBR.

\subsection{Notion de ligne d'eau de danger}

Dans les retenues où des lignes d'eau s'établissent en crue, il convient d'introduire la notion de ligne d'eau de danger en remplacement de la notion de cote de danger.

Les plans d'eau des retenues de barrage peuvent en général être considérés comme quasi horizontaux même en crue. En effet, les vitesses, donc les pertes de charge de l'écoulement, y sont faibles.

II n'en est pas de même pour les retenues étroites, c'est-à-dire des retenues caractérisées par une faible largeur et/ou une faible profondeur aboutissant à des vitesses d'écoulement significatives. Les niveaux de la rivière à l'amont du barrage y sont en pente, et ceci d'autant plus que le débit de crue est important.

Ceci n'a en général que peu d'importance pour les retenues étroites avec berges naturelles non submersibles.

II n'en est pas de même pour les aménagements du type "barrages mobiles en rivière » faisant l'objet du paragraphe 4.3.6, dont la retenue est généralement munie de digues latérales présentant un risque de rupture par submersion. II convient alors de caler ces digues par rapport à des lignes d'eau en crue et non par rapport à des cotes fixes.

Les notions de plus hautes eaux (cf. 1.1.3) et de cote de danger (cf. 1.1.4), correspondant aux situations exceptionnelle et extrême, doivent alors être remplacées par celles de :

- plus haute ligne d'eau ;

- ligne d'eau de danger, qui vont alors être dimensionnantes.

On notera que les barrages en rivière destinés à maintenir des niveaux suffisants pour la navigation sont ouverts en crue et n'ont en général pas d'endiguement amont barrage. La notion de ligne d'eau de danger est alors sans objet.

Au paragraphe 4.3.6, il est proposé de considérer les aménagements « barrages en rivière » dans leur ensemble, y compris les zones de déversements qu'il convient de bien connaître, voire de favoriser dans les zones de moindre vulnérabilité. L'insubmersibilité des digues devra en revanche être garantie dans les zones de forte vulnérabilité. 
Ceci nécessite donc l'établissement des lignes d'eau nommées ci-avant, ligne d'eau de danger en particulier, et ceci assez précisément. L'établissement de ces lignes d'eau est assez délicat :

- difficulté d'étalonnage des modèles mathématiques ou physiques, les débits correspondant n'ayant jamais été observés ;

- influence du transport solide en crue : les phénomènes suivants peuvent apparaître, pouvant modifier notablement les lignes d'eau en crue, jouant dans un sens ou dans l'autre :

- pavage, dépavage, consolidation des matériaux,

- dépôts ou affouillements, généralisés ou localisés,

- autocurage de la retenue en crue ${ }^{14}$, liée aux phénomènes cités au premier alinéa,

- apparition de dunes pouvant augmenter très notablement la rugosité ${ }^{15}$;

- revanche : la revanche à prendre en compte, par rapport aux vagues par exemple, peut être différente le long des digues suivant l'orientation de celles-ci par rapport au vent, comme le montre la figure 5.1:

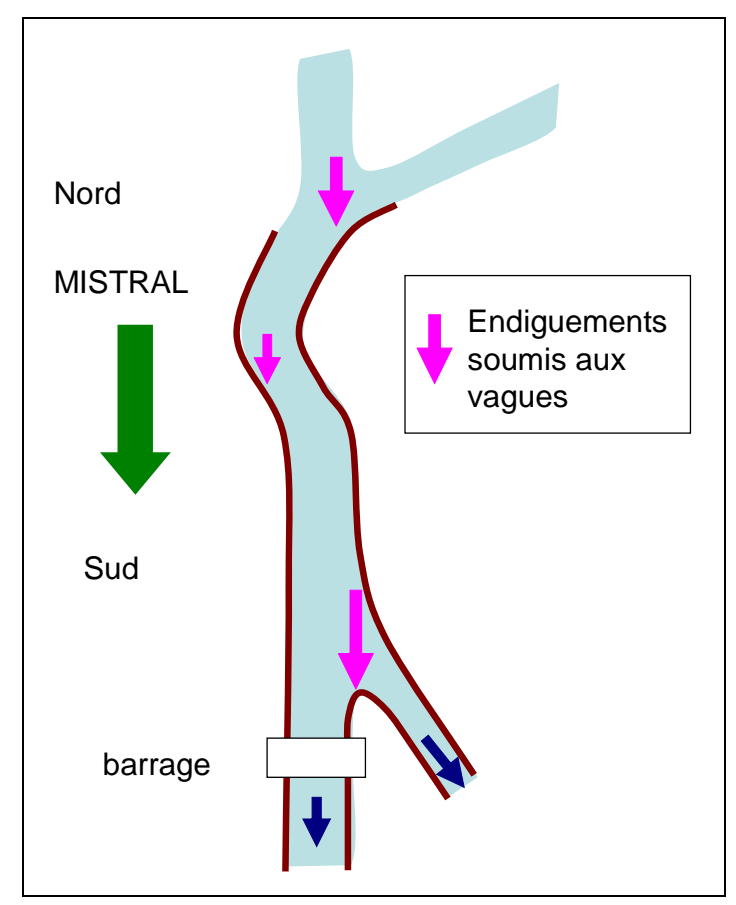

Figure 5.1 - Exemple schématique des digues du Rhône soumises aux vagues en cas de mistral

La cote des digues doit donc être ajustée en fonction de ce critère.

\section{Conclusion :}

Pour les barrages en rivière avec retenue étroite et endiguement amont, le but est d'établir les différentes lignes d'eau en crue et en particulier la ligne d'eau de danger, en tenant compte des différents phénomènes cités qui peuvent intervenir en crue, associés à un calcul d'incertitudes, de manière à :

\footnotetext{
${ }^{14}$ Pour l'aménagement CNR de Bourg lès Valence, des dépôts importants ont été observés dans I'Isère à son débouché dans la retenue de l'aménagement. Des études sur modèle physique ont montré qu'en crue, le dépôt était autocurant et in fine n'aggravait pas les lignes d'eau.

${ }^{15}$ Travaux de Marcel Ramette EDF LNH. Le coefficient de Strickler peut diminuer de 30\% en cas de dunes. Il est fortement recommandé aux exploitants d'effectuer des profils en long dans la retenue autour de la pointe de crue pour détecter les dunes et en mesurer amplitude et longueur d'onde, les dunes disparaissant en décrue.
} 
- ne jamais déverser sur les digues insubmersibles qui protègent les zones de forte vulnérabilité ;

- garantir en revanche la submersion aux endroits prévus à cet effet, ceux-ci servant de soupape de sécurité.

Les crues et débordements en résultant auront alors un caractère plus prévisible facilitant la gestion de crise et l'organisation des secours.

II est donc proposé ce thème comme axe de recherche. Celui-ci sera d'autant plus fructueux s'il peut prendre un cas concret d'aménagement avec barrage mobile en rivière comme exemple. La recherche s'appuiera sur la bibliographie existante, des simulations sur modèle mathématique à fond mobile adapté 1,2 ou $3 \mathrm{D}$, voire essai sur modèle physique, des mesures in situ en crue sur la rivière ou le fleuve.

Une publication sera effectuée conduisant à un guide de bon dimensionnement des aménagements avec barrage mobile en rivière, sous l'égide du CFBR.

\subsection{Prise en compte des incertitudes pour le choix de l'aléa hydrologique de référence}

La répartition habituelle des rôles repose d'une part sur le choix d'une probabilité annuelle de défaillance comme référence du dimensionnement, et d'autre part sur un calcul hydrologique donnant la «meilleure » estimation possible de cet aléa de référence, avec une information sur les incertitudes associées à cette estimation.

La probabilité de référence est fonction de la classe de l'ouvrage, elle-même liée au type d'ouvrage et aux conséquences possibles en aval. L'inventaire des pratiques internationales (cf. 4.2 et annexe 1) montre une forte disparité sur le choix de cet aléa de référence. Les recommandations françaises sont présentées au chapitre 4.3.3.

La question du mode de prise en compte de l'incertitude d'estimation de l'aléa hydrologique dans le choix du débit de référence est complexe. Dans l'absolu, la meilleure approche résulte d'un calcul économique visant à minimiser le coût global : coût de l'ouvrage + coût des dommages résiduels (en cas de dépassement de la crue de projet). Le second coût est obtenu en pondérant les dommages de toutes les crues $q$ supérieures à la crue de référence $Q_{\text {ref }}$ avec leur densité de probabilité :

$$
\operatorname{CDR}\left(Q_{\text {ref }}\right)=\int_{Q_{r e f}}^{+\infty} \operatorname{Dommage}(q) \cdot \frac{\partial F}{\partial q}(q) \cdot d q
$$

Il est possible de raffiner le calcul en considérant l'incertitude hydrologique (distribution sur le vecteur $\square$ des paramètres de la distribution des crues) :

$$
\operatorname{CDR}\left(Q_{r e f}\right)=\int_{Q_{r e f}}^{+\infty} \int_{\rho} \text { Dommage }(q) \cdot \frac{\partial F}{\partial q}(q \mid \rho) \cdot f(\rho) d q d \rho
$$

En pratique, on est vite confronté au problème d'attribution d'un coût aux pertes en vies humaines. Une première approche consiste à retenir la crue maximale possible pour justement ne pas avoir à se prononcer sur une acceptation d'un risque résiduel en terme de décès. En réalité, ce choix conduirait à des valeurs de référence extrêmement élevées, très incertaines et disproportionnées par rapport aux valeurs de référence pratiquées pour 
d'autres risques (naturels ou industriels). Dans la pratique, les approches de type PMP/PMF par maximisation de l'aléa reposent sur un passage implicite de la crue maximale possible à la crue maximale probable, en introduisant une part de subjectivité dans le choix de l'état initial de saturation du bassin versant et du rendement des pluies en débit. Cette crue maximale probable résulte de choix non clairement affichés et l'absence de normalisation de la méthode PMP/PMF a conduit en France à ne pas utiliser cette méthode.

Une seconde réponse consiste à combiner de façon forfaitaire aléa de référence et incertitude associée, en raisonnant par exemple sur la borne supérieure de l'intervalle de confiance $(a ̀) 70,90,95 \%$...) de la crue de référence (probabilité annuelle de dépassement $10^{-3}$ ou $10^{-4}$ ). Cette approche retenue aujourd'hui pour la sûreté des installations nucléaires vis-à-vis du risque hydrologique repose sur une grande part de subjectivité dans le choix de la largeur de l'intervalle de confiance. Elle suppose par ailleurs une certaine homogénéité dans le calcul des incertitudes. À ce jour, les incertitudes d'échantillonnage sont assez bien connues, à l'inverse de celles liées au modèle hydrologique.

Une troisième réponse consiste à raisonner sur la distribution prédictive :

$$
F(\text { Qref })=\int_{0}^{Q_{\text {ref }}} \int_{\rho} \frac{\partial F}{\partial q}(q \mid \rho) f(\rho) \cdot d q \cdot d \rho
$$

au lieu de la distribution centrale obtenue avec le jeu de paramètres estimé $\hat{\rho}$ :

$$
\hat{F}(\text { Qref })=\int_{0}^{Q_{\text {ref }}} \frac{\partial F}{\partial q}(q \mid \hat{\rho}) \cdot d q
$$

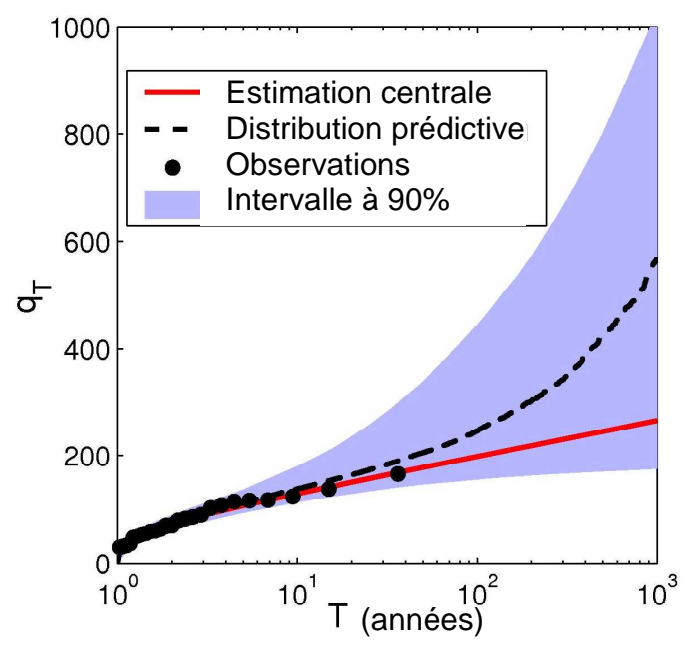

Figure 5.2 - Différence entre distribution centrale et distribution prédictive

Les différences entre les distributions sont faibles pour les quantiles courants pour lesquels les incertitudes sont réduites, mais elles deviennent significatives pour les crues rares (cf. fig. 5.2). La distribution prédictive intègre les incertitudes. Son calcul suppose la connaissance de ces incertitudes. Des applications hydrologiques sont en cours (cf. projet ExtraFlo) pour montrer l'intérêt et le caractère applicable de la distribution prédictive.

Des applications simplifiées d' « ingénierie » de cette approche ont été proposées et utilisées (Deroo, 2006). 
Une dernière réponse consiste à raisonner par analyse de sensibilité et à tester l'impact d'une surestimation (ou d'une sous-estimation) de l'aléa hydrologique sur le coût de l'ouvrage et les conséquences en cas de défaillance (crue supérieure à la crue de référence) en terme de dommages et pertes de vies humaines. On raisonne ici en valeurs relatives, de façon à évaluer l'intérêt d'un dimensionnement plus sécuritaire en rapport avec les avantages attendus. Aux États-Unis, Bowles (2008) a introduit la notion de coût disproportionné pour la réduction des conséquences.

Au final, la prise en compte des incertitudes pourrait déboucher sur le choix d'une probabilité annuelle de défaillance plus faible, si les incertitudes sont trop élevées (par rapport à une situation standard), en relation avec les conséquences importantes liées à ces incertitudes.

\section{3. État initial de remplissage du barrage et volume de la crue de dimensionnement ou de la crue extrême}

Un premier niveau d'analyse du risque de défaillance du fonctionnement du barrage peut se faire en raisonnant sur le débit de pointe de la crue, en supposant un niveau initial de la retenue à la cote de retenue normale. II est en général adapté au cas des barrages avec un très grand volume de retenue. Mais il est nécessaire d'affiner l'analyse en raisonnant sur la chronologie de l'événement hydrologique et en intégrant le fonctionnement hydraulique de la retenue via un bilan entrée-sortie, qui peut induire un diagnostic différent de celui obtenu en régime permanent à la pointe de crue. Deux conclusions opposées sont possibles, avec une marge de sécurité additionnelle liée à un stockage d'une partie du volume de la crue et à une réduction de la pointe de crue, ou au contraire un risque accru lié à la succession rapprochée de plusieurs épisodes et à un niveau initial de la retenue supérieur à la cote de retenue normale pour l'épisode le plus critique (Carvajal et al., 2009).

Aujourd'hui, pour des questions de temps de calcul, on raisonne sur un événement hydrologique de référence, avec la modélisation hydraulique de la retenue sous hypothèse de niveau initial de la retenue et d'hydrogramme de crue de référence. Une étude statistique multi-variée peut permettre d'apprécier le risque d'un niveau initial de retenue supérieur à la cote de retenue normale, si à l'expérience il existe une liaison entre le débit de base de la crue et son débit de pointe ou volume. Sachant que l'on s'intéresse aux événements extrêmes, il y a toutefois peu de chance que l'on dispose d'un nombre suffisant d'observations pour évaluer correctement ce risque additionnel. En l'état, une analyse de sensibilité peut être réalisée sur l'impact du choix du débit de base sur le fonctionnement hydraulique de la retenue (pointe et volume étant fixés), à argumenter ensuite en fonction d'informations générales sur la genèse hydro-météorologique des événements et une expertise sur la succession d'épisodes de crue.

La construction de l'hydrogramme de crue nécessite de définir précisément son mode de rattachement à une période de retour $T_{\text {ref }}$ de référence. Une première approche consiste à raisonner sur le débit de pointe $Q_{\max }\left(T_{\text {ref }}\right)$ et à faire un certain nombre d'hypothèses sur la forme de l'hydrogramme. Une expression analytique peut être retenue (Sherman, 1932 ; Roche, 1963 ; SCS, 2003...), calée à l'aide d'informations sur les hydrogrammes de crue observés : volume de la crue, durée totale de la crue, temps de montée, rapport débit de pointe sur volume de crue, débit de base... La difficulté réside dans la variabilité des formes de crue pour les événements courants, liée à une combinatoire importante sur la variabilité temporelle des averses, la contribution différenciée des sous-bassins versants, le rendement très variable de la pluie en débit suivant l'état d'humidité des sols, la genèse de la crue (crue nivale, pluviale...). Pour les événements extrêmes, la dispersion est moins forte du fait qu'une crue extrême résulte de la conjonction d'une pluie forte et d'un état initial propice à un écoulement rapide. La forme de la crue est alors la signature du bassin versant, avec un temps de réaction moyen et une forme moyenne de crue. II n'en reste pas moins plusieurs 
possibilités d'arriver au même débit de pointe, avec des formes de crue plus ou moins accentuées.

La notion de volume de crue n'est pas simple à définir : dans le cas de crues à plusieurs pics, le positionnement du début de la crue est délicat ; par ailleurs, la fin de la crue n'est pas très marquée sur les bassins à décrue lente. Pour éviter l'arbitraire dans le choix du début et de la fin de la crue, il est possible de définir la crue comme la période pendant laquelle le débit dépasse un seuil de référence. Cette solution peut dans certains cas être inappropriée lorsque ce seuil est trop élevé par rapport au débit de base (troncature d'une partie de l'épisode de crue) ou au contraire en prolongeant trop loin la fin de la crue. Une meilleure solution consiste à ne pas définir un volume de crue, ni une date de début et de fin de crue, mais à raisonner sur le débit moyen maximal $V_{d}$ de la crue, sur une durée $d$ donnée. De façon analogue aux courbes intensité-durée-fréquence pour les averses, on peut définir les courbes débit-durée-fréquence (QdF). Un point quelconque $\mathrm{V}_{d}(T)$ des courbes $\mathrm{QdF}$ correspond à un quantile de débit moyen maximal calculé sur la durée $d$ et de période de retour $T$. Le débit de pointe $Q_{\max }$ correspond au cas limite du débit $V_{d}$ pour $d=0$. Javelle (2001) a proposé une formulation assez simple des courbes QdF (modèle QdF convergent) à partir du débit de pointe et d'un paramètre $\square$ homogène à une durée :

$$
V(d, T)=\frac{Q_{\max }(T)}{1+d / \Delta}
$$

Cette relation a pu être étendue aux courbes $Q d F$ en débit seuil (débit $Q d$ dépassé de façon continue pendant la durée $d$ ) :

$$
Q(d, T)=\frac{Q_{\max }(T)}{(1+d / \Delta)^{2}}
$$

On peut alors construire un hydrogramme synthétique monofréquence (HSMF) qui présente la propriété d'être homogène en fréquence, quel que soit le débit utilisé pour le caractériser :

- $1^{\text {ère }}$ itération (calcul d'une forme moyenne de crue) : la méthode consiste (Garçon et al., 2002) à normaliser les hydrogrammes observés par le débit moyen maximal sur une durée de référence (caractéristique du bassin versant), et à faire coïncider la pointe de crue. La figure 5.3 présente un exemple d'hydrogramme moyen normalisé dont on conservera la forme pour la montée de crue ; 


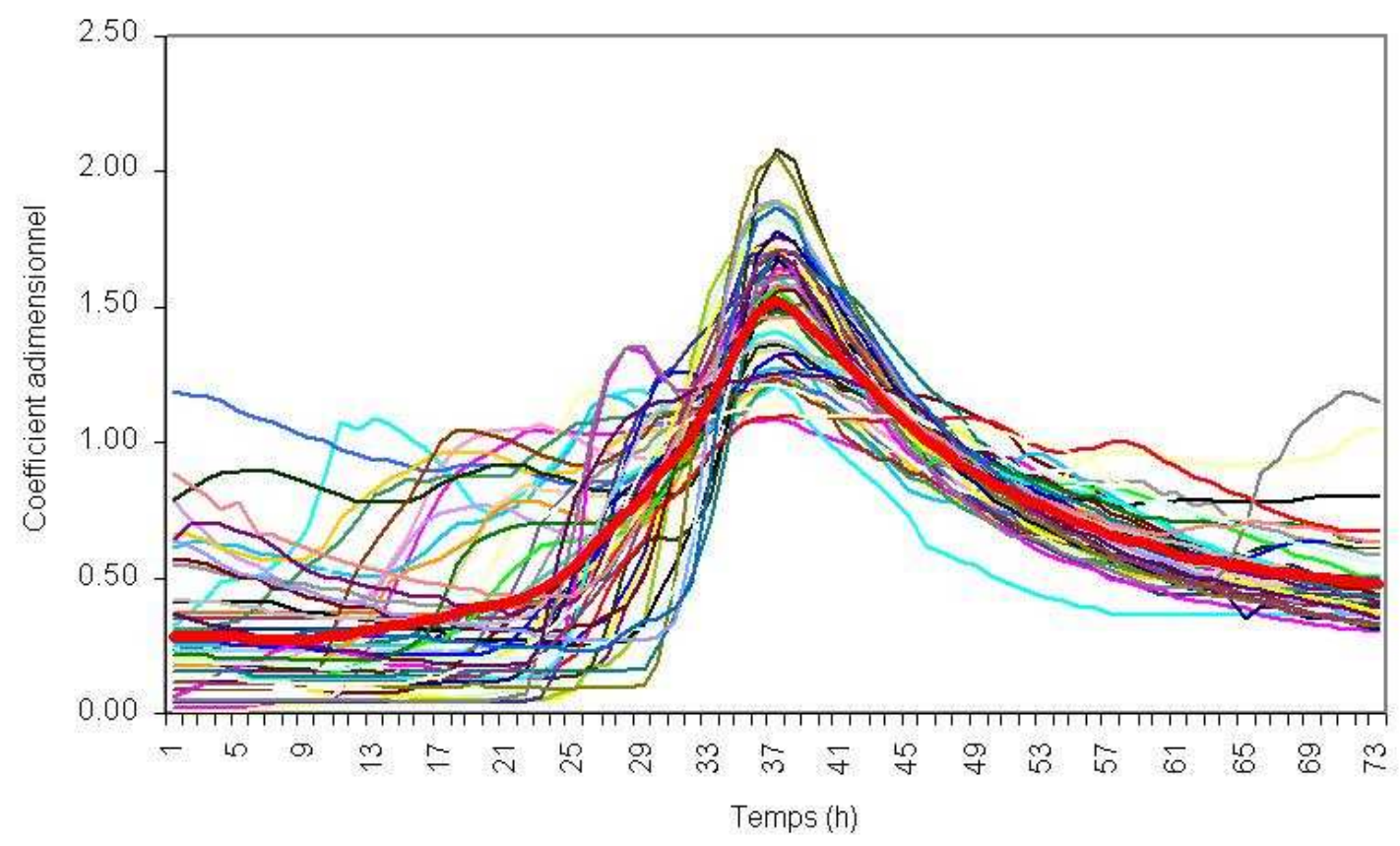

Figure 5.3 - Hydrogramme moyen issu des hydrogrammes normalisés sur la Valserine à Lancrans

- $2^{\text {ème }}$ itération (correction de la décrue de façon à rester homogène en fréquence) : la phase de décrue est constituée pas à pas, à partir des quantiles en débit seuil $Q d(T)$. Sur la figure 5.4, le point $n^{\circ} i$ ( $i$ varie de 2 à 4) est obtenu en reportant en ordonnée la valeur du quantile $Q d_{i}(T)$, et en abscisse la durée $d_{i}$ à partir de la branche de montée de l'hydrogramme. Le débit $Q d_{i}(T)$ est en effet le débit dépassé de façon continue pendant la durée $d_{i}$. Galéa et Prudhomme (1994) ont montré la cohérence de l'hydrogramme HSMF avec les courbes QdF en débit moyen maximal Vd. L'intégration de l'hydrogramme HSMF pour une durée $d$ donnée donne un débit $V d_{\text {HSMF. }}$ Les deux auteurs ont montré que la période de retour de ce débit, déduite des abaques QdF en débit moyen $V d$, était très voisine de la période de retour $T$ de l'hydrogramme HSMF construit à partir des débits seuils $Q d(T)$.

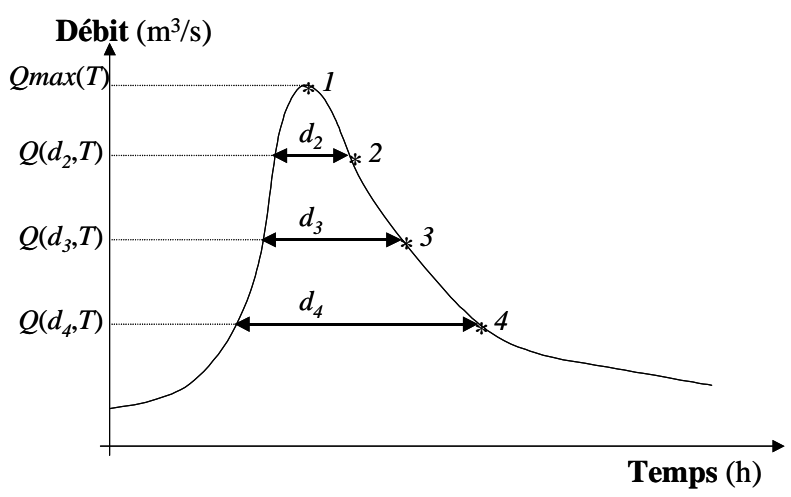

Figure 5.4 - Construction d'un hydrogramme synthétique monofréquence HSMF

Des variantes permettent par ailleurs d'introduire des contraintes supplémentaires dans la construction de l'hydrogramme (Le Clerc, 2004), pour respecter les gradients de montée de crue (cas où l'on s'intéresse à la sécurité d'un ouvrage d'écrêtement pour lequel le mode de gestion est très sensible à la rapidité de montée des eaux) ou des formes spécifiques d'hydrogrammes de crue (à deux bosses) en classant les événements suivant une typologie 
de formes (coefficient d'asymétrie et écart-type). Lorsque le régime des crues met en évidence une forte variabilité des formes de crue, due par exemple à une origine météorologique différente (crue océanique, crue cévenole) ou une genèse physique différente (crue nivale, crue pluviale), il est possible de raisonner par scénario de crue, chacun étant spécifique d'une typologie de forme de crue. La figure 5.5 illustre le cas d'un événement avec deux pointes successives rapprochées, qui peut être plus pénalisant qu'une crue à une seule pointe de plus fort débit maximal.

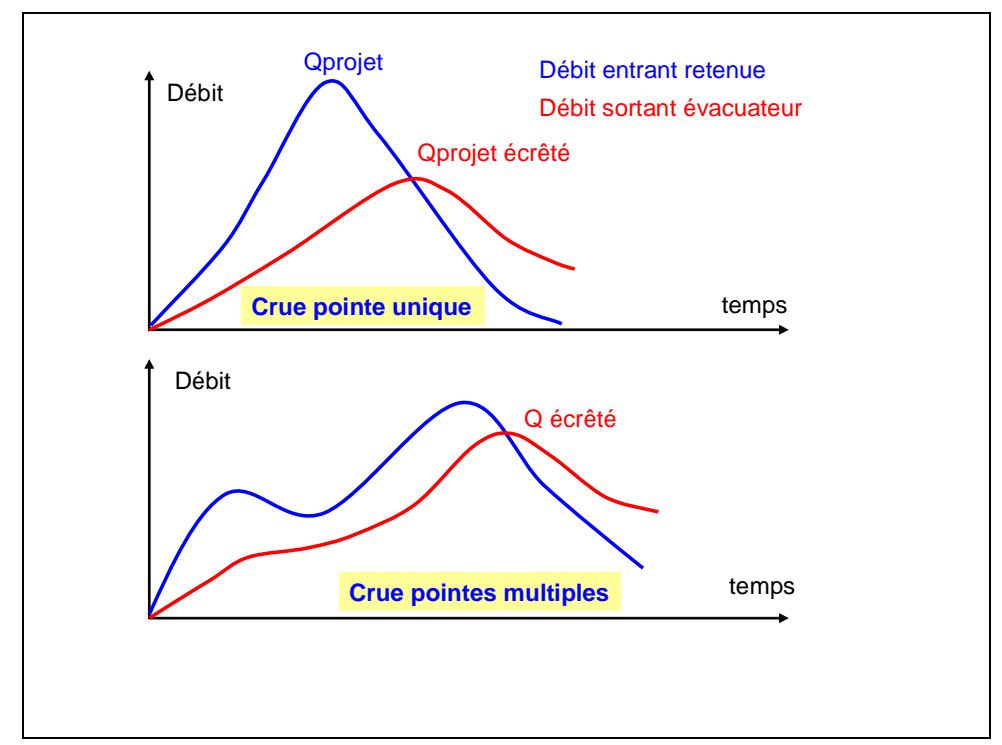

Figure 5.5 - Laminage de deux crues de référence : pointe unique et pointes rapprochées

Enfin, une dernière approche consiste à raisonner par simulation hydrologique et à tester le fonctionnement hydraulique de la retenue sur une longue série hydrométrique simulée de plusieurs milliers d'années. Celle-ci contient toute la variabilité possible des formes de crue et de succession d'épisodes.

Au lieu de définir d'abord une crue de projet, puis ensuite de l'injecter dans un modèle hydraulique pour en déduire le niveau atteint dans la retenue, on simule le comportement hydraulique de l'ouvrage pour toute une série de crues obtenues par une approche de simulation (cf. chapitre 2). Il est alors possible d'étudier la distribution de fréquence des cotes atteintes dans la retenue et d'en déduire une cote de projet, associée à une probabilité au non-dépassement cible. Lavabre et al. (2009) ont présenté une application de cette approche, à l'aide du simulateur Shypre, pour les barrages de Sénéchas et de Conqueyrac sur le Gard.

La question de l'analyse des concomitances a concerné ici le risque d'avoir à la fois un niveau initial élevé de la retenue et un fort volume de crue, du fait notamment d'une succession rapprochée de pointes de crue. Elle se pose également dans le cas d'ouvrages situés en bord de mer, où il faut gérer le risque de concomitance entre une crue et une surcote marine, ou bien dans le cas de très grands bassins versants où l'on envisage une concomitance des crues de plusieurs affluents. Là encore, les analyses multi-variées peuvent être utilisées, mais elles restent d'un usage délicat en extrapolation. Calées sur les observations disponibles avec peu de référence d'événements concomitants, elles donneront des résultats différents sur les événements extrêmes suivant la fonction de dépendance introduite dans la modélisation multi-variée. La recherche d'information régionale sur des événements historiques concomitants peut orienter le choix empirique de situations de concomitance. 


\subsection{Estimation des crues extrêmes}

La notion de crue de danger fait référence à une situation extrême issue de la conjonction d'une crue extrême et du disfonctionnement des ouvrages d'évacuation. Dans le tableau 4.7, il est proposé de se référer pour la crue extrême à une probabilité annuelle de dépassement qui va de $10^{-3}$ à $10^{-5}$, suivant la classe de barrage. II faut rappeler ici que les méthodes d'extrapolation les plus récentes développées par les hydrologues (méthodes Shypre et Schadex) ne vont pas au-delà de $p=10^{-4}$. Un exercice de simulation a été réalisé par P. Arnaud (Irstea Aix) pour analyser la variabilité du ratio :

$$
R=Q\left(10^{-5}\right) / Q\left(10^{-4}\right)
$$

Le modèle Shypre, poussé au-delà de son domaine d'utilisation standard, donne la valeur de ce ratio par pixel de $1 \mathrm{~km}^{2}$, sur l'ensemble de la métropole. La carte produite montre une valeur $R$ assez stable (de l'ordre de 1,2 à 1,3) sur la moitié sud de la France (diagonale Bayonne-Strasbourg) concernée par des pluies fortes. Sur la moitié nord, avec un régime des pluies de type océanique, le ratio $R$ varie dans une fourchette assez large (entre 1,3 et $3,0)$. II peut paraître paradoxal que le ratio d'accroissement des débits extrêmes soit plus fort pour les secteurs où la pluie est moins intense. L'interprétation est que sur les bassins soumis à une pluie océanique, le modèle pluie-débit utilisé pour les simulations a une forte capacité d'interception, qui dépasse largement l'intensité des pluies courantes. Dans ce cas, la distribution des débits ne décolle que pour les pluies extrêmes, alors que, pour les bassins soumis à un régime de pluies méditerranéennes, la saturation du bassin est déjà atteinte pour une probabilité $p=10^{-4}$.

Ce résultat, un peu contre-intuitif mais plausible, reste lié à l'utilisation du modèle Shypre. Avec un autre référentiel d'étude (Gradex, Schadex), il n'est pas certain qu'utiliser un ratio $R$ plus fort sur les bassins « mous » que sur les bassins « réactifs » ne soit pas trop pénalisant. De fortes incertitudes existent actuellement sur la modélisation de la capacité d'interception des bassins « mous » pour les crues extrêmes.

En l'état actuel des connaissances, il est proposé d'approcher la crue de danger de la manière suivante :

- conserver le domaine ultime d'estimation des crues extrêmes à une probabilité de dépassement $p=10^{-4}$ et estimer la crue $Q\left(10^{4}\right)$ par une des méthodes basées sur l'information pluviométrique (cf. section 2.7) ;

- appliquer un coefficient multiplicatif sur la crue $Q\left(10^{4}\right)$, fonction de la cible à atteindre pour la crue de danger: $Q_{\text {danger }}=C \cdot Q\left(10^{4}\right)$

avec :

$C \approx \operatorname{Ln}\left(10^{5}\right) / \operatorname{Ln}\left(10^{4}\right)$ arrondi à 1,3 pour les barrages de classe $A$,

$C \approx \operatorname{Ln}\left(1 / 3.10^{-5}\right) / \operatorname{Ln}\left(10^{4}\right)$ arrondi à 1,15 pour les barrages de classe $B$.

Dans les zones soumises à faible précipitation (au Nord de la diagonale BayonneStrasbourg) et pour des bassins avec une forte capacité d'interception, un argumentaire spécifique est à développer pour chaque bassin. On pourra raisonner en majorant la pluie $P\left(10^{4}\right)$ ou le scénario de pluies prévalant à une crue de débit $Q\left(10^{4}\right)$ par le coefficient multiplicatif $C$, et la transformer ensuite avec un modèle déterministe pluie-débit dans les conditions hydrologiques identifiées pour la crue de débit $Q\left(10^{4}\right)$.

Outre cette approche simple, l'évaluation de la crue de danger pourra être critiquée / modulée par le jugement de l'hydrologue et par la prise en compte d'approches historiques ou naturalistes. Dans le futur, une solution pourra être de majorer la crue $Q\left(10^{4}\right)$ à l'aide d'un 
calcul en mode prédictif (cf. 5.2). II reste encore à progresser dans la connaissance et la modélisation des incertitudes.

\subsection{Nouvelles problématiques posées par l'approche des états limites ultimes}

La compétence des ingénieurs a été jusqu'à une époque récente mobilisée essentiellement pour concevoir et réaliser des ouvrages ou organes apportant une réponse « totalement fiable » par rapport aux fonctionnalités requises et ceci dans le domaine d'utilisation qui leur était fixé.

Au niveau de la conception, cette notion de « totale fiabilité » passait par :

- la prise en compte de marges de sécurité plaçant l'ouvrage ou l'organe suffisamment loin de sa limite de rupture ou de dysfonctionnement pour que les phénomènes physiques encore mal cernés soient inclus dans de telles marges de sécurité ;

- le choix de types d'ouvrages ou d'organes permettant de se ramener à des situations bien connues, faisant l'objet d'abaques ou de formules de dimensionnement éprouvées ou ayant fait l'objet de réalisations similaires à partir desquelles on pouvait réaliser des extrapolations fiables.

Dans ce contexte, l'ingénieur devait refuser d'être mis dans la situation où, par exemple sous la pression d'un donneur d'ordre souhaitant faire des économies, telle disposition ne serait adoptée que si on pouvait prouver que sans cette disposition il y aurait rupture de l'ouvrage ou de l'organe, ou non réponse aux fonctionnalités requises.

Par ailleurs, on se posait rarement la question du comportement de l'ouvrage ou de l'organe en dehors du domaine d'utilisation qui lui était fixé.

Le contexte réglementaire tend d'une part à introduire dans tous les domaines la notion d'état limite ultime s'approchant de la rupture et d'autre part à caractériser et prendre en compte dans la démarche de conception les probabilités de rupture ou de dysfonctionnement des ouvrages et des organes.

Globalement, ceci oblige le concepteur à s'intéresser à des nouvelles situations non considérées jusqu'ici.

Un bon exemple pour le domaine qui nous intéresse dans le présent document est le cas d'un barrage-voûte et de sa capacité à passer les crues :

- dans le contexte précédent, l'évacuateur était conçu pour éviter la surverse sur le corps du barrage pour la crue de projet fixée. La fosse de dissipation était conçue pour assurer sa fonction en cas de fonctionnement normal, c'est-à-dire un écoulement dont l'impact était dirigé vers le centre de la vallée et suffisamment loin du pied du barrage, en adoptant si nécessaire des déflecteurs dont l'effet pouvait être prévu avec précision. On pouvait par exemple se contenter de s'assurer que la fosse d'érosion limite à long terme correspondant à la crue de projet, déterminée par des formules empiriques et confirmée par une modélisation physique avec fond érodable, ne mettait pas en péril la stabilité du barrage et de sa fondation ;

- la prise en compte des situations extrêmes proposée dans les présentes recommandations amènera l'ingénieur à évaluer quel niveau de surverse est acceptable par ce barrage pour éviter des travaux de confortement très ou trop coûteux. Par 
exemple pour ce qui concerne l'hydraulique, cela passera par l'évaluation de la capacité de la fondation en pied du barrage et sur toute la hauteur de ses appuis à résister à l'écoulement pendant la durée limitée de la crue extrême. On introduit ici deux nouvelles problématiques, d'une part la prise en compte d'un type d'écoulement moins bien contrôlé et d'autre part la notion de durée de l'événement.

Même si l'approche est nouvelle, en l'état actuel, l'ingénieur n'est pas totalement démuni pour aborder les nouvelles problématiques exposées ci-dessus. Certaines situations sont déjà étudiées aujourd'hui pour les besoins de projets réels, des travaux de recherche ont déjà été publiés et des retours d'expérience sont ou peuvent être établis à partir des ouvrages existants.

D'une manière générale, les modélisations numériques et physiques, chacune avec ses limites qui doivent être connues de l'utilisateur, permettent d'apprécier les débitances, les lignes d'eau y compris les ondes le long des coursiers, les pressions dynamiques ainsi que les érosions dans des situations non prévues par les formules et les abaques de dimensionnement classiques. Ces outils utilisés jusqu'ici principalement pour modéliser des conditions de crue de projet sont néanmoins disponibles pour apprécier des conditions d'écoulement relevant des situations extrêmes.

Des dispositions simples, souvent déjà mises en œuvre sur des ouvrages existants et dont l'effet peut être estimé avec les outils disponibles, sont déjà à notre disposition pour améliorer la tenue des ouvrages dans les conditions d'événements extrêmes, par exemple :

- surélévation de la crête d'un barrage béton près des rives, pour éloigner les écoulements des fondations en rive en cas de surverse ;

- plus généralement, construction de massifs brise-charge ou déflecteurs sur les fondations en rive pour contrôler les écoulements ;

- clouage des écailles rocheuses dans les zones potentiellement sujettes à des écoulements en cas d'événement extrême ;

- ouvrages fusibles pour faire en sorte que les écoulements liés aux événements extrêmes se forment sur les ouvrages ou dans les zones où leurs conséquences sont les moins importantes ou peuvent être contrôlées.

Cependant, il reste nécessaire de continuer les efforts pour :

- formuler des conclusions à partir des publications et des retours d'expérience disponibles dès maintenant ;

- poursuivre les recherches et l'analyse de cas existants pour progressivement affiner notre connaissance des phénomènes physiques mis en jeu à la limite de la rupture des ouvrages pour apporter des éléments permettant d'améliorer la formulation.

Nous donnons ci-après quelques exemples où des éléments de réponse existent mais sont perfectibles, pour traiter la question des états limites ultimes :

Pour ce concerne les érosions, et en particulier l'érosion des massifs rocheux :

- dans la conception des fosses de dissipation des évacuateurs de crues construits sur des rivières puissantes, dans des pays où la réglementation fait appel à des crues de type extrême ou en l'absence de réglementation, les concepteurs sont depuis longtemps amenés à apprécier l'ampleur envisageable des érosions à l'échelle de temps d'une ou plusieurs fortes crues ;

- il existe dès aujourd'hui des évacuateurs dont le coursier est constitué par des chenaux simplement excavés dans le rocher et un retour d'expérience peut être tiré de ces exemples. Des auteurs ont travaillé sur la vitesse d'érosion des massifs rocheux et ont 
proposé des conclusions en fonction des caractéristiques géomécaniques classiques entrant dans la classification des masses rocheuses (cf. congrès CIGB de Durban, 1994) ;

- la tenue des rives rocheuses sous l'effet des écoulements en cas de surverse du barrage concerne en grande partie la tenue d'écailles rocheuses soumises à des sous-pressions dues à l'introduction de pressions dynamiques. La stabilité de ces écailles peut être étudiée de manière classique à partir de la géométrie de ces écailles qui peut être déterminée, et à partir d'une valeur maximale des sous-pressions qui peut être évaluée d'après des mesures sur des modèles physiques ou d'après les vitesses des écoulements qui est limitée par la charge disponible ;

- des travaux ont déjà été menés pour améliorer la connaissance des pressions dynamiques pouvant intervenir en fond des fosses de dissipation et tenter de formuler des conclusions ;

- la tenue des parements béton ou même des blindages sujets à des écoulements rapides et/ou chargés en sédiments (par exemple en cas de l'utilisation en urgence d'une vanne de fond en cas de crue extrême) fait déjà l'objet de publications et se pose déjà dans des cas réels, mais il reste encore à formuler des conclusions générales à partir des informations existantes qui proviennent de sources diverses, et à poursuivre les recherches sur des matériaux performants à cet égard.

\subsection{Détermination de l'occurrence des crues de danger par la méthode des dommages incrémentaux}

\subsubsection{Introduction}

Historiquement, cette méthode a été élaborée lors des débats de la commission publique spéciale d'expert, diligentée par le gouvernement de la province du Québec (Canada) à la suite des crues dévastatrices de 1996 dans la région du Saguenay-Lac St Jean, ayant entraîné la rupture de digues, notamment celles des barrages Hydro Québec de ChuteGarneau \& Pond-Arnaud.

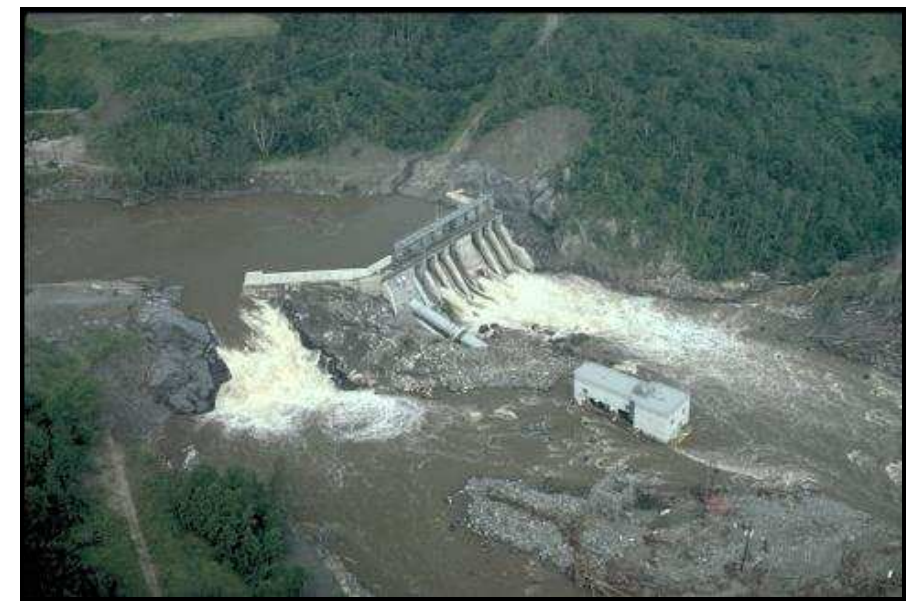

Photo 5.1 - Brèche de 20 m dans le mur en aile du barrage de Jonquière 


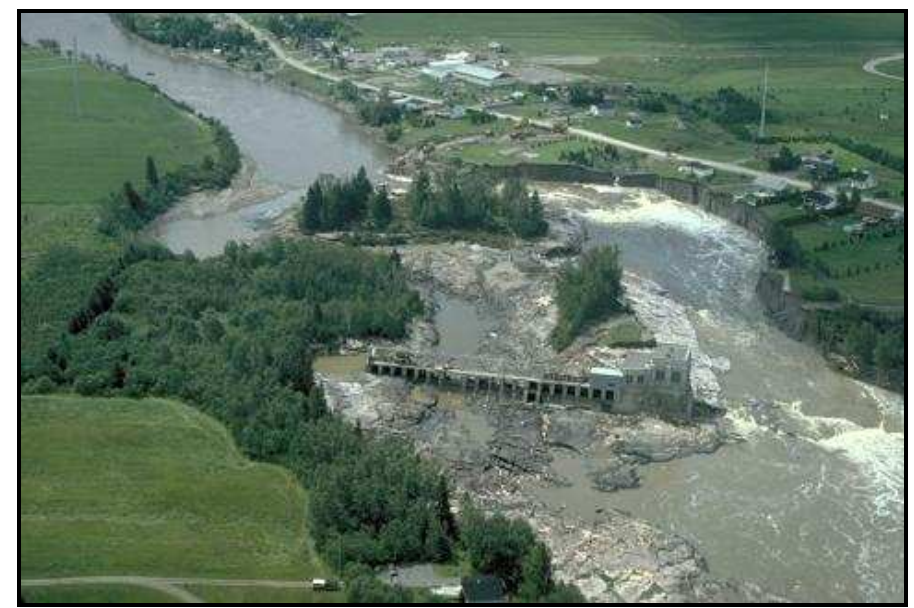

Photo 5.2 - Barrage de Chute Garneau - Chenal de contournement

Le but recherché était d'estimer la crue extrême qu'un barrage devait passer dans des conditions ultimes de fonctionnement (équivalent d'une crue de danger). L'approche utilisée est une approche par les risques aval, indépendamment de la classe intrinsèque du barrage (ou de son importance).

Cette méthode a été introduite dans la loi québécoise sur la sécurité des barrages de 2000 et son règlement (équivalent de décret) de 2002, ce dernier ayant fait l'objet d'une révision en 2011, pour en supprimer certains biais.

On signalera que cette méthode ne vise à déterminer que la crue de danger du barrage (cf. crue de sécurité dans le règlement québécois) : la notion de crue de projet ou de dimensionnement n'est alors pas un critère de dimensionnement de l'évacuateur de crues et ne figure pas dans la loi québécoise.

Le texte ci-après présente les principes de la méthode utilisée au Québec (paragraphes 5.6.2 à 5.6.4).

\subsubsection{Principe de la méthode telle qu'appliquée au Québec}

La détermination de la crue de danger est fondée sur le niveau des dommages constatés à l'aval du barrage, du fait de sa rupture en crue, indépendamment de sa vulnérabilité ou de sa classe. L'exposé de la méthode présentée ici s'appuie sur des extraits des articles du règlement québécois qui y font référence.

Extrait de l'article 17 du règlement québécois : "Le niveau des conséquences d'une rupture du barrage est déterminé selon les caractéristiques du territoire qui serait affecté par la rupture, localisé, sauf exception, en aval du barrage et en considérant, parmi plusieurs scénarios de rupture, celui qui entraîne le niveau de conséquences le plus important. Ces caractéristiques sont évaluées en termes de densité de population et d'importance des infrastructures et services qui seraient détruits ou lourdement endommagés en cas de rupture ».

On ne s'intéresse donc ici qu'à l'incrément de dommages subis à l'aval d'un barrage, entre les situations sans rupture et celles avec rupture: ce sont les dommages incrémentaux induits par la rupture de l'ouvrage. 
Une table en annexe du règlement dresse les niveaux de conséquence :

- les densités de population concernées par cet incrément de niveau d'inondation vont de zones non habitées à des zones de plus de 10000 habitants ;

- les infrastructures concernées sont de plusieurs natures: infrastructures industrielles, infrastructures de transport (routes, voies ferrées), services publics tels que les hôpitaux;

- les niveaux de conséquence sont qualifiés en fonction de la gravité des dommages: minimal, faible, moyen, important, très important, considérable.

\subsubsection{Détermination de l'incrément de hausse de niveau au- delà duquel on impute les dommages à la rupture du barrage}

La rédaction initiale de l'article 17 mentionnait expressément le niveau au-delà duquel on pouvait estimer un impact significatif de la rupture du barrage au regard des populations et des infrastructures et services:

"Les dommages dont l'augmentation est appréciée en vertu du présent article sont ceux susceptibles de survenir en aval du barrage. II n'y a pas d'augmentation des dommages attribuables à la rupture d'une crue donnée, aux termes du présent article, lorsque le rehaussement du niveau d'eau causé par la rupture du barrage ne dépasse pas $60 \mathrm{~cm}$ ».

Ce seuil ne figure plus dans l'article modifié de 2011. Dès lors, Hydro Québec recommande les modalités suivantes: "Lorsque le terrain d'un résident est inondé en son point le plus bas sur le pourtour de la résidence, les personnes de la résidence sont considérées affectées par la rupture du barrage dès qu'il y a un rehaussement de $15 \mathrm{~cm}$ et plus.

Il y a de lourds dommages ou la destruction de résidence, d'infrastructure et de service lorsque le rehaussement provoqué par la rupture d'un barrage est de $30 \mathrm{~cm}$ et plus ».

\subsubsection{Détermination des zones de dommages incrémentaux}

L'article 18 du règlement précise le mode de détermination :

"La délimitation du territoire qui serait affecté par la rupture du barrage ainsi que l'identification des caractéristiques de ce territoire sont définies sur la base d'une étude de rupture du barrage, à laquelle sont jointes des cartes d'inondation. Cette étude consiste, par des méthodes reconnues, en une évaluation détaillée des conséquences de la rupture au moyen de la délimitation précise du territoire affecté et de l'identification des caractéristiques de ce territoire. Cette étude implique l'examen de divers scénarios de rupture, en conditions normales et en période de crues. Elle comporte une description des hypothèses et des méthodes utilisées pour le choix des scénarios étudiés ainsi que pour la détermination de l'onde de submersion, de son temps de propagation et de l'étendue du territoire affecté. Dans les scénarios prévoyant la rupture du barrage en période de crues, le territoire affecté correspond à celui dont l'inondation est exclusivement attribuable à la rupture du barrage ».

Les articles 21 à 23 font le lien entre niveau des conséquences et occurrence de la crue de danger à considérer dans le dimensionnement des évacuateurs et stabilité du barrage :

Article 21 : Les caractéristiques de tout barrage doivent assurer au minimum la résistance aux crues de danger mentionnées dans le tableau ci-dessous en tenant compte du niveau des conséquences d'une rupture du barrage. Toutefois, si les conséquences de la rupture ont été évaluées sur la base d'une étude de rupture, le niveau à considérer aux fins du 
tableau ci-dessous est celui qui, parmi les scénarios de rupture en période de crues, entraîne le niveau de conséquences le plus important ».

\begin{tabular}{|l|l|}
\hline $\begin{array}{l}\text { Niveau } \\
\text { des } \\
\text { conséquences }\end{array}$ & $\begin{array}{l}\text { Crue de danger } \\
\text { (probabilité d'occurrence) }\end{array}$ \\
\hline Minimal ou Faible & $1: 100$ ans \\
\hline $\begin{array}{l}\text { Moyen ou } \\
\text { Important }\end{array}$ & $1: 1000$ ans \\
\hline Très important & $1: 10000$ ans ou $1 / 2$ CMP \\
\hline Considérable & $\begin{array}{l}\text { Crue maximale probable } \\
\text { (CMP) }\end{array}$ \\
\hline
\end{tabular}

L'article 21 impose donc une corrélation arbitraire entre le niveau des conséquences et la crue de sécurité (danger) à retenir, et ce indépendamment de la situation de crue qui a conduit à ce niveau de conséquences. Par exemple, à l'aune de l'article 21 , si la rupture d'un barrage lors d'une crue centennale occasionnait un niveau de conséquences jugé "considérable» (cf. tableau), la crue de sécurité (danger) à considérer dans le dimensionnement de l'ouvrage et son évacuateur de crues serait de niveau CMP.

Ce « biais », introduit par l'article 21, a été révisé via un nouvel article 23 remanié en 2011 :

Article 23 : "La crue de danger d'un barrage peut être moindre, sans toutefois être inférieure à la crue centennale, s'il est démontré qu'une rupture lors d'une telle crue entraînerait un niveau de conséquences inférieur à celui utilisé aux fins de l'application du tableau cidessus ».

\subsubsection{Traduction graphique de détermination de la crue de danger}

On propose ici une illustration de l'application des articles 21 et 23 du règlement québécois, au travers de trois exemples. Les trois barrages pour lesquels on se propose de déterminer la crue de danger par la méthode des dommages incrémentaux ont des typologies très différentes:

- le barrage «bleu » retient un réservoir de grand volume et sa rupture entraîne, quelles que soient les situations à cet instant, de grands dommages incrémentaux à l'aval ;

- le barrage «jaune » est un barrage de faible capacité, de type barrage mobile, les dommages incrémentaux consécutifs à sa rupture se trouvent diminués fortement avec l'importance de la crue (car pour un barrage mobile sans capacité, le passage d'une crue CMP ou équivalent dans la vallée n'est pratiquement pas affecté par la tenue ou la rupture de l'ouvrage) ;

- le barrage « vert » est un cas intermédiaire.

Pour le barrage «jaune », l'application directe de l'article 21 conduit à une crue de danger d'occurrence 1000 ans (flèches 1 et 2). L'application de l'article 23 corrige cette valeur, les dommages incrémentaux de la rupture du barrage pour cette crue conduisant à des niveaux de dommages inférieurs à ceux causés par la crue inférieure : la crue de danger dans ce cas est alors la crue de retour 100 ans (flèches 3, 4 et 5). 


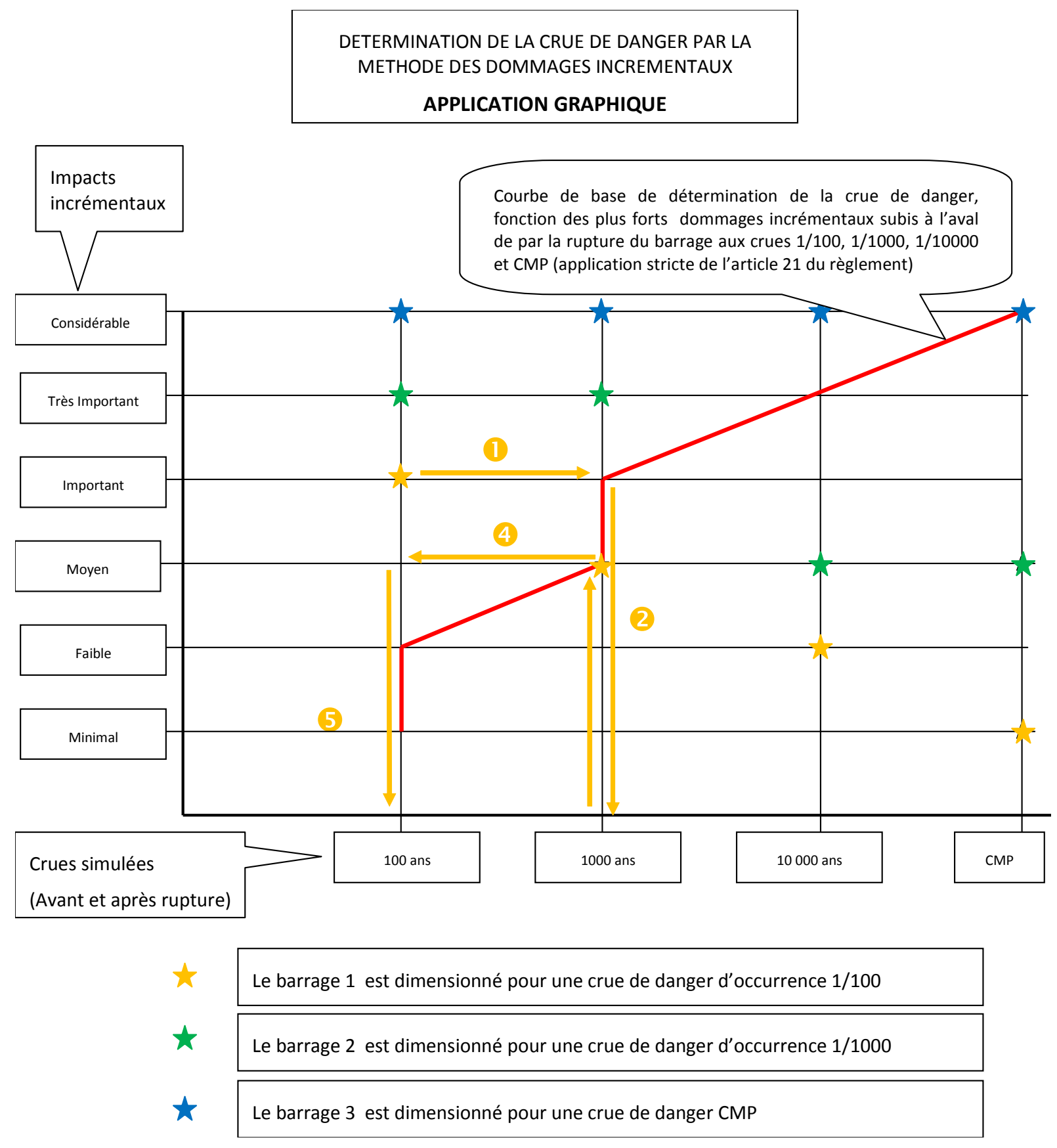

\subsubsection{Conduite pratique de la méthode}

La méthodologie de conduite d'une étude de détermination d'une crue de sécurité (danger) par les dommages incrémentaux est la suivante:

- calculs hydrologiques et hydrauliques de référence : évaluation des débits des crues, débitance des organes d'évacuation des crues, débits et formes de brèche ;

- choix des hypothèses et des méthodes utilisées pour les scénarios de rupture et pour la détermination de l'onde de submersion résultante: mode de rupture progressive ou instantanée, critère de rupture au pic de crue ou à l'atteinte du couronnement, etc. ;

- élaboration d'un modèle numérique hydraulique du cours d'eau (généralement 1D sauf si insuffisance démontrée) en aval du barrage et calé sur les laisses de crues ; 
- étude des ondes de rupture du barrage propagées sur différents scénarios hydrologiques (différents temps de retour, typiquement : 100 ans , 500 ans, 1000 ans, 10000 ans voire au-delà) ;

- étude des mêmes scénarios hydrologiques (mêmes temps de retour) mais cette fois sans rupture du barrage:

- établissement des cartes d'inondation pour tous les scénarios étudiés, avec et sans rupture de l'ouvrage (de préférence au moyen d'un SIG) ;

- pour chaque scénario hydrologique étudié : délimitation précise du territoire affecté par la rupture du barrage (c'est-à-dire frange du territoire dont l'inondation est exclusivement attribuable à l'incrément d'aléa dû à la rupture). Pour chaque scénario hydrologique étudié: identification des caractéristiques de cette frange de territoire (zone des dommages incrémentaux) affectée par la rupture du barrage (sauf exception comme les prises d'eau potable), en termes de densité de population et d'importance des infrastructures et services qui seraient détruits ou lourdement endommagés ; un niveau de conséquences peut donc être estimé pour chaque scénario hydrologique examiné ;

- identification, parmi les scénarios hydrologiques étudiés, de celui qui entraîne le niveau de conséquences le plus important ;

- application des articles 21 et 23 du règlement québécois pour déterminer la crue de danger (cf. schéma précédent).

\subsubsection{Applicabilité de la méthode en France}

La méthode des dommages incrémentaux repose fondamentalement sur des principes conventionnels, touchant globalement au niveau de risque contre lequel la communauté souhaite se protéger et en particulier au niveau d'acceptabilité/refus des populations au regard du risque industriel consécutif à un risque naturel. II conviendrait donc de débattre des sujets suivants pour une application en France :

- la forme et la cinétique des brèches ;

- la détermination physique de l'incrément de niveau d'inondation imputable, lors d'un épisode de crue, à la seule rupture du barrage ;

- la nature des dommages subis par cet incrément d'inondation et sa gradation ;

- la règle de dimensionnement des évacuateurs de crues en fonction de ces dommages.

\subsubsection{Forme et cinétique des brèches}

La forme et la cinétique des brèches ou des ruptures des barrages sont des paramètres déterminants dans la forme de l'hydrogramme du lâcher d'eau et donc des surélévations des niveaux d'eau incrémentaux à l'aval de l'ouvrage et leurs gradients. II conviendrait donc de fixer des règles partagées selon les types d'ouvrages et les différentes situations rencontrées.

Pour le cas des barrages en béton ou maçonnerie, les brèches sont en général instantanées ou importantes, de largeur nettement supérieure à la hauteur: le débit résultant, très important pour un ouvrage haut, peut même atteindre plusieurs milliers de $\mathrm{m}^{3} / \mathrm{s}$ pour un ouvrage de $15 \mathrm{~m}$ de hauteur. Elles ne constituent pas nécessairement un effacement total de l'ouvrage.

La submersion d'un barrage en remblai entraîne une vitesse de l'eau faible en crête mais beaucoup plus forte en pied aval où s'amorce généralement la brèche, qui progresse ensuite vers la crête, puis s'ouvre à l'amont ; l'ouverture se fait d'abord sur une largeur assez faible, d'où un débit initial (en $\left.\mathrm{m}^{3} / \mathrm{s}\right)$ de l'ordre de $\mathrm{H}^{2,5}(\mathrm{H}$ en $\mathrm{m}$ - hauteur du barrage dans la zone de 
la brèche) avec une vitesse modérée de l'eau (en $\mathrm{m} / \mathrm{s}$ ) de l'ordre de $\sqrt{ } \mathrm{H}$; la brèche peut s'élargir rapidement si le remblai est peu cohésif et mal compacté, assez lentement pour un remblai argileux moderne.

À titre d'exemple, pour de vieux barrages de 25 à $30 \mathrm{~m}$ de hauteur (Banquiao en Chine en 1975, Machu en Inde en 1979), le débit a donc été de l'ordre de $80000 \mathrm{~m}^{3} / \mathrm{s}$ et des brèches se sont ouvertes sur plus de $500 \mathrm{~m}$ de longueur.

Des ruptures de barrages plus hauts, plus modernes ont conduit à des débits relativement plus faibles (Téton aux USA en 1975, $80000 \mathrm{~m}^{3} / \mathrm{s}$ pour $80 \mathrm{~m}$ de hauteur - Euclides d'Acuna au Brésil en 1977, $40 \mathrm{~m}$ de hauteur, brèche triangulaire).

Le débit d'une brèche peut être assez faible pour des barrages modernes en remblai mais plusieurs brèches peuvent se créer si la crue est longue (grand bassin versant).

À titre d'illustration, les hydrogrammes liés à l'onde de rupture sont schématisés sur la figure 5.6 ci-dessous pour différents types de barrages et des volumes de crue proches du volume stocké.

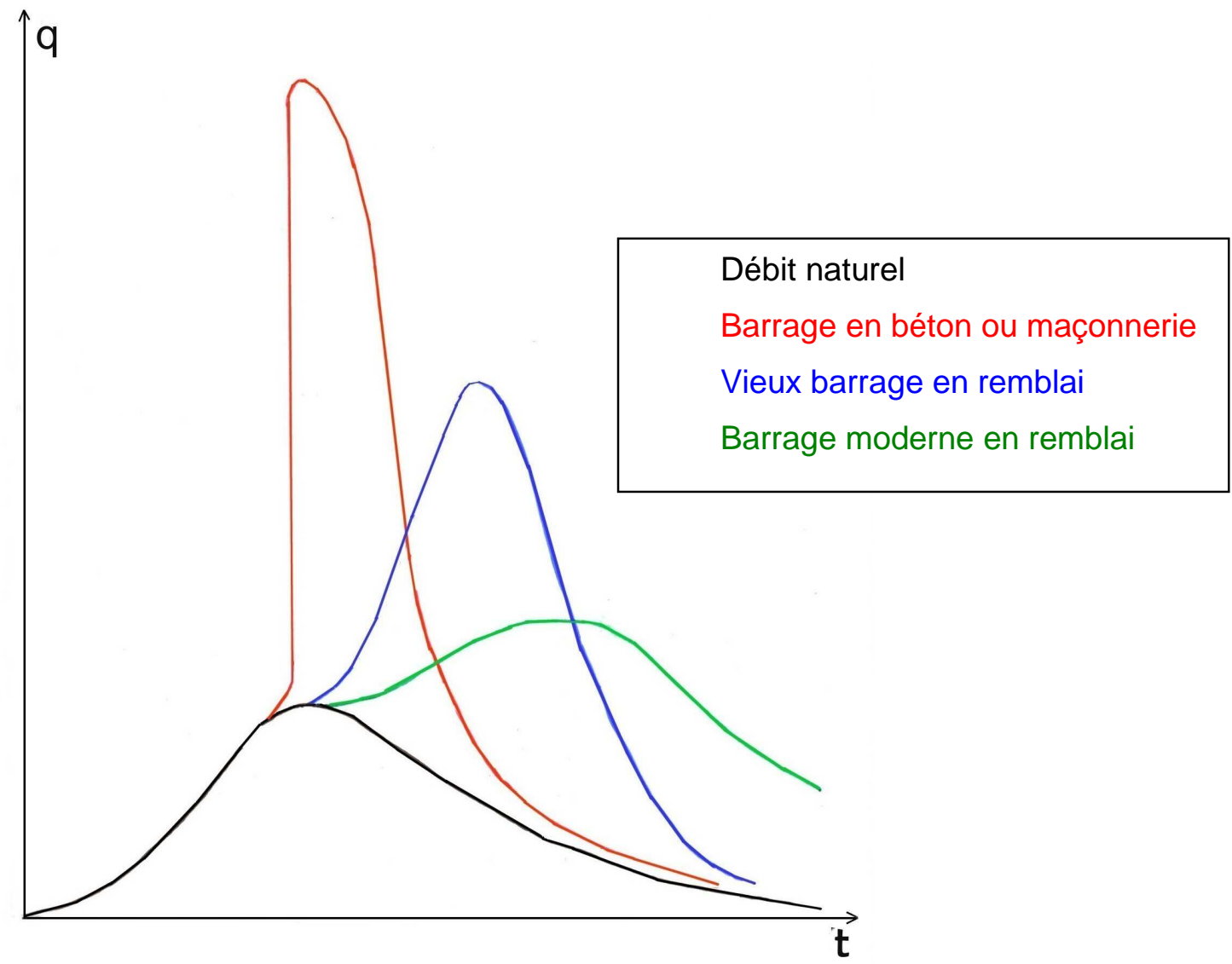

Figure 5.6 - Hydrogrammes des ondes de rupture

\subsubsection{Détermination de l'incrément}

La détermination de l'incrément de hauteur d'eau doit être discutée. Comme exposé en 5.6.2.2, cette valeur a fait l'objet de débats et le texte réglementaire québécois a évolué sur ce point. Pour sa détermination, il convient sans doute de bien distinguer les dommages matériels, dont la couverture relève de l'assurance, des dangers pour les tiers. Sur ce dernier point et bien que les contextes soient de nature très différente, on pourrait se référer notamment aux travaux menés dans le cadre de l'application de la circulaire interministérielle 
du 13 juillet 1999, relative à la sécurité à proximité et à l'aval des barrages en exploitation, où la détermination de paramètres repères tels que le niveau d'eau, son gradient de montée et la vitesse du courant ont fait l'objet d'études spécifiques sur l'atteinte directe à l'intégrité des personnes présentes dans le lit des rivières, lors d'opérations de lâchers d'eau depuis le barrage.

Ces études incrémentales (comparatif entre une situation de crue sans rupture et avec rupture) permettraient de préciser la notion de dangerosité d'un barrage, au-delà des critères actuels synthétisés par la valeur de l'expression $\mathrm{H}^{2} \sqrt{ } \mathrm{V}$ différenciant les classes des barrages français, et ceci particulièrement pour les barrages mobiles, dont le volume de retenue est faible par rapport à des débits de crue notables.

\subsubsection{Nature des dommages subis par cet incrément d'inondation et sa gradation}

La France est un pays dont la densité de population est bien supérieure à celle du Québec, constat qu'il convient de prendre en compte, tout en régionalisant ce point. II conviendrait d'adapter les termes réglementaires québécois de conséquences « minimal, faible, moyen, important, très important, considérable » à un référentiel compatible avec le corpus de textes réglementaires, guides ou études existant en France. Un lien naturel avec les études de dangers de barrage, réglementairement introduites en France par le décret 2007-1735 du 11 décembre 2007, pourrait être établi, et étendu à cet égard à l'ensemble des barrages de classes $\mathrm{C}$ et $\mathrm{D}$.

\subsubsection{Règle de dimensionnement des évacuateurs de crues fonction de ces dommages}

La transformation du niveau de dommages incrémentaux, induits par la rupture d'un barrage lors d'une crue, en occurrence de la crue de danger de cet ouvrage et de son évacuateur est là encore un sujet délicat, touchant à la fois à l'acceptabilité sociétale d'un niveau de risque et à l'examen détaillé et complexe des effets combinés des causes et conséquences d'une crue importante sur les dommages, à l'aval comme à l'amont d'un barrage (cas de rupture de digues de protection entraînant elles-mêmes des inondations subies ou intentionnelles). Là encore, les principes proposés doivent être examinés au regard d'autres dispositions réglementaires ou recommandées pour le dimensionnement d'autres infrastructures potentiellement dangereuses ou celles concernant les ouvrages de protection eux-mêmes.

\subsubsection{Suites à donner}

Le Groupe de travail recommande d'engager une réflexion au niveau français sur cette approche. Il est proposé d'établir notamment un retour d'expérience sur son application, ses limites, ses difficultés de mise en œuvre au Québec, afin d'examiner l'éventualité d'une application sur des ouvrages français, ses niveaux de transposition et d'adaptation. 


\section{Annexe 1 : Pratiques étrangères}

\section{Australie - New South Wales}

Dam safety committee guidelines

DSC13 : consequence categories for dams

DSC11 : acceptable flood capacity for dams

\section{Éléments de terminologie}

Population at Risk (PAR) : toutes les personnes qui seraient directement exposées à la crue, si elles ne prenaient aucune action pour évacuer ; itinérants sont pondérés par une probabilité de présence. Dam crest flood (DCF) : crue qui amène à la crête (a remplacé la notion de IFF : Imminent Failure Flood, semble-t-il car IFF a été un concept ambigu et difficile à manier.

\section{Classification des barrages}

\begin{tabular}{|c|c|c|c|c|}
\hline \multirow{2}{*}{$\begin{array}{c}\text { Population at } \\
\text { Risk (PAR) } \\
\text { (Note 7) }\end{array}$} & \multicolumn{4}{|c|}{ Severity of Damage and Loss } \\
\hline & Negligible & Minor & Medium & Major \\
\hline$<1$ & Very low & Very Low & Low & Significant \\
\hline 1 to 10 & $\begin{array}{c}\text { Low } \\
\text { (Notes 1, } 4 \& \text { \&) }\end{array}$ & $\begin{array}{c}\text { Low } \\
\text { (Notes } 4 \& 5 \text { ) }\end{array}$ & $\begin{array}{c}\text { Significant } \\
\text { (Note 5) }\end{array}$ & $\begin{array}{l}\text { High C } \\
\text { (Note 6) }\end{array}$ \\
\hline 10 to 100 & \multirow[t]{3}{*}{ (Note 1) } & $\begin{array}{l}\text { Significant } \\
\text { (Notes 2\& } 5 \text { ) }\end{array}$ & $\begin{array}{l}\text { High C } \\
\text { (Note 6) }\end{array}$ & $\begin{array}{l}\text { High B } \\
\text { (Note 6) }\end{array}$ \\
\hline 100 to 1000 & & \multirow[t]{2}{*}{ (Note 2) } & $\begin{array}{l}\text { High A } \\
\text { (Note 6) }\end{array}$ & $\begin{array}{l}\text { High A } \\
\text { (Note 6) }\end{array}$ \\
\hline$>1000$ & & & (Note 3) & Extreme \\
\hline
\end{tabular}

- Les risques sont évalués de manière incrémentale, par rapport à la situation de référence qui est la crue générée sans rupture du barrage. Cependant, le risque total est également considéré.

- Il est également demandé de commenter, outre la PAR, une estimation du nombre potentiel de victimes.

\section{Crue a CONSIDERER}

\section{Critères de dimensionnement}

\begin{tabular}{|l|l|}
\hline \multicolumn{1}{|c|}{ FCC RATING (i) } & \multicolumn{1}{c|}{ FLOOD OR AEP (ii) } \\
\hline Extreme \& High A & PMF (reservoir full) \\
\hline High B & Max AEP (PMP Design Flood or $10^{-6}$ ) (iii) \\
\hline High C & Max AEP (PMP Design Flood or $10^{-5}$ ) (iii) \\
\hline Significant & $10^{-4}$ \\
\hline Low & $10^{-3}$ \\
\hline Very Low & No requirements (iv) \\
\hline
\end{tabular}

Revanche

- A dimensionner au cas par cas, de manière conservative, pour tenir compte de : tassements, incertitude sur les crues, en particulier pour les barrages en remblais.

- Barrage en remblais : revanche minimale 0,3 $\mathrm{m}$ à $0,6 \mathrm{~m}$ selon la classe

- Barrage en béton : on peut admettre revanche nulle, et parfois négative.

\section{Prise en compte de la disponibilité des évacuateurs}

Les calculs doivent tenir compte des conditions les plus défavorables en termes de : gestion de la retenue, comportement des ouvrages. En particulier, pour les barrages vannés, le Comité demande une analyse de fiabilité.

Cote initiale de la retenue : dans certains cas, il est souhaitable d'examiner la probabilité conjointe entre cote initiale de retenue et débit de crue. 


\section{Divers}

Analyse préliminaire de sensibilité : Le Comité doit être averti immédiatement si DCF est de probabilité supérieure à $10^{-4}$ pour les barrages Extreme et High $\mathrm{A}$ et $10^{-3}$ pour High $\mathrm{B}$, High $\mathrm{C}$ et Significant. Dans le cas des barrages en remblai, cela impose à une mise en sécurité immédiate. 


\section{Brésil}

\begin{tabular}{|r} 
Référence et date \\
Document ELETROBRAS/ Comite Brasileiro de Barragens
\end{tabular}

«Criterios de Projeto Civil de Usinas Hidrelectricas » Oct 2003

Eléments de terminologie

Niveau Maximal en Crue du Réservoir = niveau maximum atteint lors du passage de la Crue de Projet, le réservoir étant initialement à son Niveau Normal Maximal d'Opération. Pas de définition de la Cote de Danger

Crue de Projet du Barrage: PMF ou crue de période de retour donnée, suivant classification du barrage, pour laquelle sont dimensionnés les ouvrages d'évacuation.

\section{Classification des barrages}

Classe $A$ : Barrages $\mathrm{H}>30 \mathrm{~m}$ ou dont la rupture implique un risque de pertes de vies humaines (habitations permanentes à l'aval)

Classe $B$ : Barrages $\mathrm{H}<30 \mathrm{~m}$ ou Vretenue $<50 \mathrm{hm}^{3}$, dont la rupture n'implique pas de risque de perte de vies humaines (pas d'habitations permanentes à l'aval)

\section{Type d'évacuateur}

\section{Critères de dimensionnement}

Adoption d'un évacuateur libre si Vitesse max. de montée du plan d'eau $>2 \mathrm{~m} / \mathrm{h}$

Au moins 2 vannes en cas d'évacuateur vanné

Crue de Projet du Barrage

Barrages de classe A : PMF déterminée suivant procédure de la World Meteorological Organization (1973)

Barrages de classe $B$ : crue de période de retour $T>1000$ ans, définie à partir d'une analyse de risques

Revanche minimale au-dessus du Niveau Maximal en Crue du Réservoir : 0,5 m pour les barrages en maçonnerie et béton ; $1 \mathrm{~m}$ pour les barrages en remblai

Structures de dissipation d'énergie

Préférence pour les sauts de ski et fosses d'érosion, vis-à-vis des structures à ressaut hydraulique ou

«roller buckets». Prédimensionnement des fosses d'érosion par la formule de Veronese avec coefficient dépendant de la nature du fond

\begin{tabular}{|l|}
\hline \multicolumn{1}{|c|}{ Prise en compte de la disponibilité des évacuateurs } \\
\hline- \\
\hline- \\
\hline \multicolumn{1}{|c|}{ Méthodes de calcul de la crue } \\
\hline $\begin{array}{l}\text { Obligation de réaliser une « étude de rupture des ouvrages » avec formation d'une brèche (critères de } \\
\text { dimensions et temps de formation de la brèche donnés) et propagation à l'aval de l'onde de crue } \\
\text { correspondante } \\
\text { Obligation d'élaborer un Plan d'Actions d'Urgence (PAU) basé sur l'analyse des conséquences de } \\
\text { cette propagation d'onde de crue, précisant: identification des urgences, actions préventives, } \\
\text { procédures de notification, systèmes de communication, modes d'intervention en cas de panne } \\
\text { électrique ou d'intempéries, sources d'équipements et de main d'œuvre pour interventions } \\
\text { Révisions périodiques de ce PAU, en fonction du degré de risque (5 ans au plus si risque élevé, } \\
10 \text { ans au plus si risque faible) }\end{array}$ \\
\hline
\end{tabular}




\section{États-Unis}

FEMA Federal Guidelines for Dam Safety

\section{Référence et date}

Selecting and accommodating Inflow design floods for dams, 1998 (reprinted 2004)

\section{Éléments de terminologie}

Inflow Design Flood (IDF) : la crue utilisée pour le dimensionnement des évacuateurs, pour déterminer la hauteur maximale du barrage. IDF $\leq$ PMF

Normal freeboard : crête - RN

Minimum freeboard : crête - cote atteinte pour IDF.

Intermediate freeboard : crête - cote de stockage intermédiaire (par exemple pour écrêteurs de crues)

\section{Classification des barrages}

High hazard dam : la rupture ou l'erreur de manœuvre conduisent probablement à une perte en vies humaines. La classification doit être faite en considérant le cas de rupture le plus défavorable.

\section{Critères de dimensionnement}

Crue : PMF

Une crue inférieure à la PMF peut être acceptée si les conséquences d'une rupture sont acceptables, c'est-à-dire en général si :

- il n'y a pas d'habitations humaines ou permanentes ou commerces ou industries, ou projets de développements de la sorte dans la zone qui serait inondée en cas de rupture,

- il y a des habitations humaines permanentes mais le risque incrémental (aux personnes, aux biens) est non significatif. C'est le cas par exemple des retenues à tout petit volume de stockage. En première approche, «non significatif » = « 2 feet or less », mais cela doit être justifié au cas par cas.

Dans ce cas, évaluation des probabilités des crues et analyse de risque. En tous cas, pas moins que la crue centennale.

Revanche

Il faut dimensionner la revanche normale (cote $=\mathrm{RN}$, vents très forts) et la revanche minimale (cote $=$ $\mathrm{PHE}$, vents raisonnablement possibles dans ces conditions). Ajouter tout ce qu'il faut pour tassements, séismes, impacts gravitaires, ...

\section{Prise en compte de la disponibilité des évacuateurs}

En général, cote initiale de retenue $=\mathrm{RN}$; une cote inférieure peut parfois être considérée.

Seuls les équipements dont la fiabilité est attendue sont réputés être opérationnels pour la crue. La fiabilité dépend des caractéristiques structurelles et de la disponibilité, et notamment de : disponibilité en alimentation électrique (réseau, secourue), effets des corps flottants, embâcles par la glace, accessibilité aux organes de manœuvre, aux postes de commandes, état des routes d'accès aux ouvrages, disponibilité du personnel sur site, ...

\section{Méthodes de calcul de la crue}

\section{Divers}

Contrairement aux pratiques, il est demandé de ne pas faire de distinction entre ouvrages neufs et ouvrages existants.

Les ruptures en cascades doivent être évaluées.

Un $\S$ sur le choix entre évacuateur vanné / non vanné. À noter en particulier les commentaires suivants :

quand le temps de réaction disponible est de seulement quelques heures, les barrages non vannés sont plus fiables, notamment pour les structures à fort risque aval,

la capacité des évacuateurs vannés d'abaisser rapidement le niveau de retenue peut être un avantage pour les opérations de vidange rapide.

Un § sur Service spillways / auxiliary spillways / emergency spillways. 


\section{Italie}

Article G. Galeati lors du colloque Evacuateurs

Référence et date

Loi de 1959, « règles techniques » de 1982 et l'équivalent d'une circulaire de 2004.

\section{Éléments de terminologie}

Revanche nette $=$ Crête - PHE $-1 / 2$ vague - provisions pour tassements, ...: il s'agit donc d'une revanche nette, uniquement destinée à fournir une sécurité hydrologique.

\section{Classification des barrages}

Crue

Crue de période de retour 1000 ans.

Revanche nette minimale

$1 \mathrm{~m}$ pour les barrages en maçonnerie et béton ; 1,5 à $4 \mathrm{~m}$ pour les barrages en remblai.

\section{Prise en compte de la disponibilité des évacuateurs}

Disponibilité des évacuateurs vannés : selon les « règles techniques »

Barrage en remblais : la revanche nette ne doit pas être réduite de plus de $50 \%$, et doit rester au moins égale à $1 \mathrm{~m}$, si les vannes ne sont pas manœuvrables.

Disponibilité des évacuateurs vannés : selon la directive de 2004

Evacuateurs munis de vannes automatique : réduction de $50 \%$ de la débitance ( ? texte ambigu ?).

Risques d'obstruction:

Blocage possible si la portée est inférieure à $10 \mathrm{~m}$ ou si le tirant d'air au-dessus des PHE est inférieur à $1 \mathrm{~m}$. Si le blocage est possible, alors les calculs sont faits en considérant une réduction de $50 \%$ de la débitance de l'évacuateur.

\section{Méthodes de calcul de la crue}

\section{Divers}

Évacuateurs de fond (morning glory ou similaire) : il doit être démontré qu'il n'y a pas saturation pour un niveau de retenue égal à $\mathrm{PHE}+2 / 3$ de la revanche nette. 


\section{Norvège}

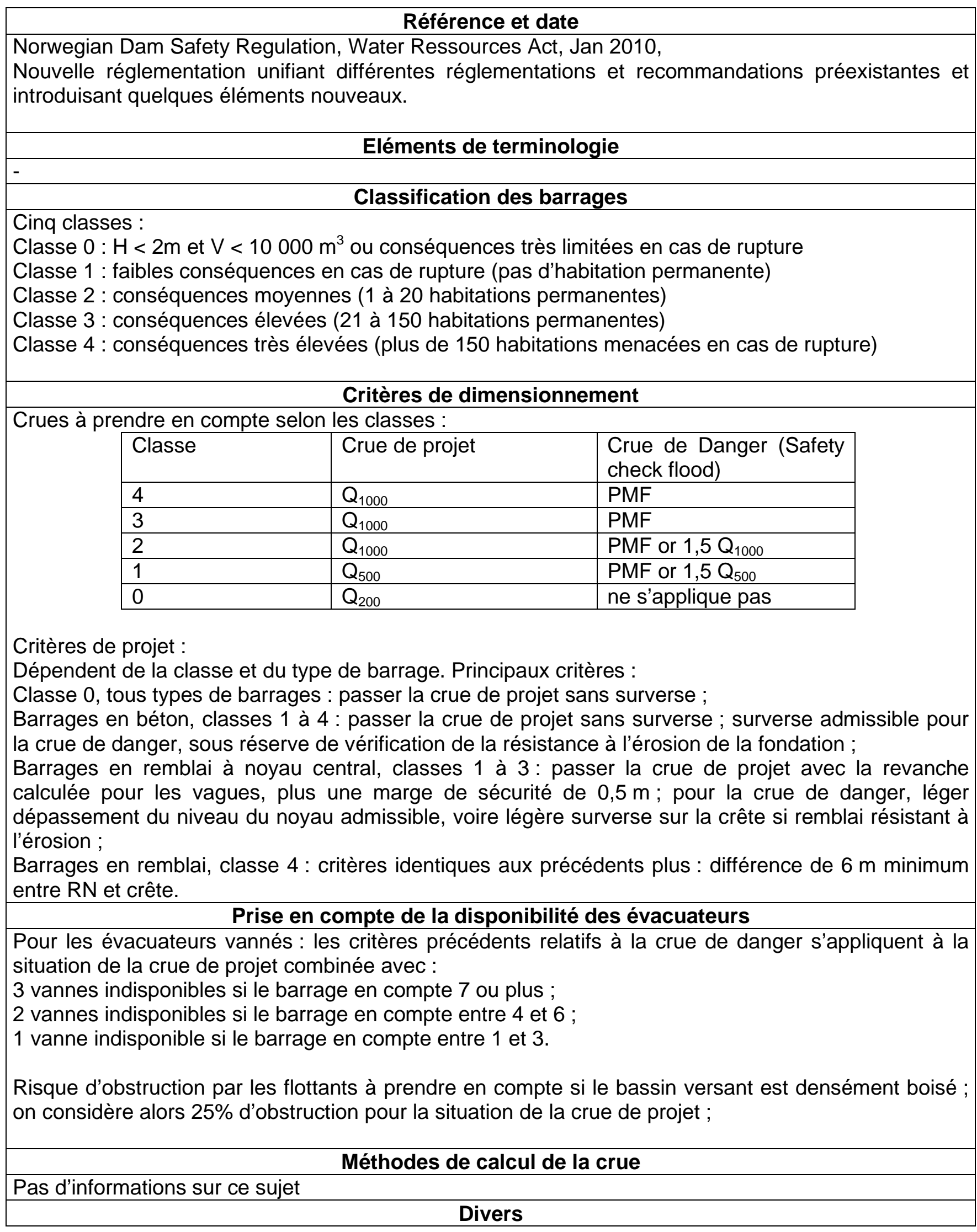




\section{Québec}

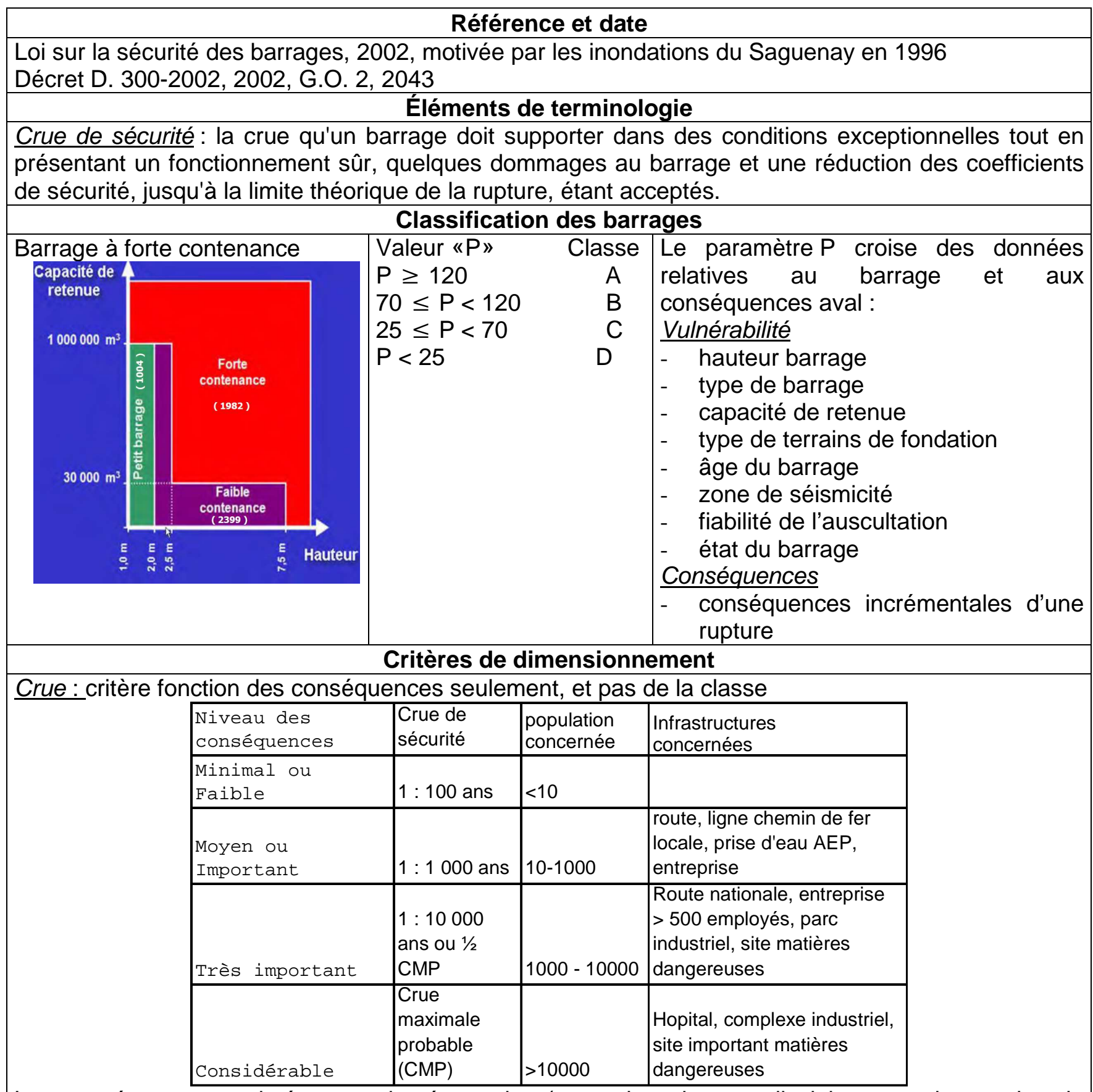

Les conséquences estimées sont incrémentales (cependant, la nouvelle loi ne mentionne plus de seuil de rehaussement en dessous duquel il n'y a pas d'augmentation des dommages attribuables à la rupture). La crue de sécurité applicable peut alors correspondre à la crue la plus faible pour laquelle il n'y a pas augmentation des dommages attribuables à la rupture, mais ne peut être inférieure à une crue centennale.

Revanche $: 1 \mathrm{~m}$ (sauf si crue $=\mathrm{CMP}$, alors pas de revanche exigée).

Pour les barrages neufs, l'étanchéité doit être portée au niveau de retenue atteint en crue de sûreté.

\section{Prise en compte de la disponibilité des évacuateurs}

$-$

\section{Méthodes de calcul de la crue}

"Des hypothèses et des méthodes réalistes et prudentes, eu égard aux règles de l'art, doivent être utilisées dans l'estimation de la crue de sécurité applicable au barrage et dans le calcul de la capacité de ce dernier de la gérer. » 


\section{Divers}




\section{Royaume-Uni}

Article A. Hughes lors du colloque Évacuateurs

\section{Référence et date}

- Reservoirs Act 1975 et propositions pour une nouvelle loi en 2010/2011 (motivée par des incidents sur des petits barrages, causés par des orages de forte intensité mais très localisés).

- Guide Floods and Reservoir Safety, 1978, mis à jour en 1989 et 1996.

\section{Éléments de terminologie}

\section{Classification des barrages}

Reservoirs Act : concerne les retenues de plus de $25000 \mathrm{~m}^{3}$

\begin{tabular}{|c|l|c|}
\hline Dam Category & \multicolumn{1}{|c|}{ Potential effect of Breach } & Reservoir Design Flood \\
\hline A & Endangers lives in a community & PMF yr \\
\hline B & $\begin{array}{l}\text { endangers lives not in a community } \\
\text { cause extensive damage }\end{array}$ & $1000 \mathrm{yr}$ \\
\hline C & $\begin{array}{l}\text { Negligible risk to life and limited } \\
\text { damage }\end{array}$ & $1000 \mathrm{yr}$ \\
\hline D & No loss of life foreseen & $150 \mathrm{yr}$ \\
\hline
\end{tabular}

Catégorie $A$ : communauté : plus de 10 personnes

Catégorie B : habitat isolé ; dommages par exemple sur routes importantes ou chemins de fer

Catégorie $\mathrm{C}$ : chemins piétons par exemple.

Propositions de changement (rapport Pitt)

- supprimer ou abaisser $\left(10000 \mathrm{~m}^{3}\right)$ le critère de volume minimal

- trois catégories de risque : High, Significant, Low ; le classement serait basé sur des facteurs combinant la probabilité de rupture et ses conséquences.

\begin{tabular}{|c|c|c|c|c|c|}
\hline \multicolumn{6}{|c|}{ Critères de dimensionnement } \\
\hline \multirow[b]{2}{*}{$\begin{array}{l}\text { Darn } \\
\text { category }\end{array}$} & \multirow[b]{2}{*}{$\begin{array}{l}\text { Potential effect of } \\
\text { a dam breach }\end{array}$} & \multirow{2}{*}{$\begin{array}{l}\text { Initial reservoir } \\
\text { condition } \\
\text { standard }\end{array}$} & \multicolumn{2}{|c|}{ Reservoir design flood inflow } & \multirow{2}{*}{$\begin{array}{l}\text { Concurrent wind speed } \\
\text { and minimum wave } \\
\text { surcharge allowance }\end{array}$} \\
\hline & & & General & $\begin{array}{l}\text { Minimum standard } \\
\text { if overtopping is tolerable }\end{array}$ & \\
\hline A & $\begin{array}{l}\text { Where a treach could } \\
\text { ondanger lives in a comnaunity }\end{array}$ & $\begin{array}{l}\text { Spilling long.term } \\
\text { average inllow }\end{array}$ & $\begin{array}{l}\text { Probsble Maximum } \\
\text { Flood (PMF) }\end{array}$ & 10,000 -year flood & $\begin{array}{l}\text { Mean annual maximum } \\
\text { hourly wind speed }\end{array}$ \\
\hline B & $\begin{array}{l}\text { Where a breach } \\
\text { (i) could endanger lives not } \\
\text { in a community or } \\
\text { (iii) could result in extensive } \\
\text { camage }\end{array}$ & $\begin{array}{l}\text { Just full } \\
\text { (i.e. no spill) }\end{array}$ & 10,000-year flood & 1000-year flood & $\begin{array}{l}\text { Mean annual maximum } \\
\text { hourly wind speed } \\
\text { Wave surcharge allomice } \\
\text { not less tha? } 0.6 \mathrm{~m}\end{array}$ \\
\hline c & $\begin{array}{l}\text { Where a treach would pose } \\
\text { negligible risk to líe ard } \\
\text { cause innited damige }\end{array}$ & Just full (i.e.ne spill) & 1000-year fleod & 150-year tood & $\begin{array}{l}\text { Mean annual maximum } \\
\text { hourly wind speed } \\
\text { Wave surcharge a lowance } \\
\text { not less than } 0.4 \mathrm{~m}\end{array}$ \\
\hline D & $\begin{array}{l}\text { Speciaj cases where nc los: } \\
\text { of life can be foroseen as a } \\
\text { resuit of a breaci and very } \\
\text { limitec additonal flood damage } \\
\text { would be caused }\end{array}$ & $\begin{array}{l}\text { Spilling long-term } \\
\text { average inflow }\end{array}$ & 150-year floed & Not applicable & $\begin{array}{l}\text { Mean annual maximum } \\
\text { hourly wind speed } \\
\text { Wave surcharge allowance } \\
\text { not less than } 0.3 \mathrm{~m}\end{array}$ \\
\hline
\end{tabular}

Prise en compte de la disponibilité des évacuateurs

Évacuateurs vannés: au moins deux vannes; si une vanne est hors-service, les autres organes doivent au moins permettre de faire passer la crue de 150 ans; si le barrage est en catégorie A, les vannes doivent être automatisées.

\section{Méthodes de calcul de la crue}

\section{Divers}




\section{Suède}

\section{Référence et date}

Swedish Guidelines for Design Flood Determination for Dams, 2007

\section{Éléments de terminologie}

$-$

Deux catégories :

\section{Classification des barrages}

I - Probabilité non négligeable de perte de vie humaine ou de blessure OU Probabilité forte de dommages économiques importants.

II - Probabilité non négligeable de dommages économiques importants.

Barrages de classe I:

- crue de projet

- revanche suffisante pour faire face au wind set-up et vagues générées par un vent de 20 à $25 \mathrm{~m} / \mathrm{s}$ Barrages de classe II :

- crue centennale

- $\quad$ analyse coût-bénéfice pour vérifier qu'il ne faut pas considérer de crues plus fortes

- revanche suffisante pour faire face au wind set-up et vagues générées par un vent de 20 à $25 \mathrm{~m} / \mathrm{s}$.

Non évoqué

Prise en compte de la disponibilité des évacuateurs

Le Guide définit entièrement la procédure de calcul des crues :

Crue de projet

- la Suède est découpée en régions ; pour chaque région, une pluie de référence (24h) est donnée, qui correspond approximativement à la pluie maximale mesurée dans la région,

- la pluie est ensuite corrigée en fonction de l'altitude et de la taille du bassin,

- la fonte des neiges est prise en compte (valeurs préfixées),

- le ruissellement est de $100 \%$

Cela est réputé fournir une pluie de période de retour 10000 ans au moins.

Crue centennale

Calculée par ajustement des débits mesurés au barrage. 


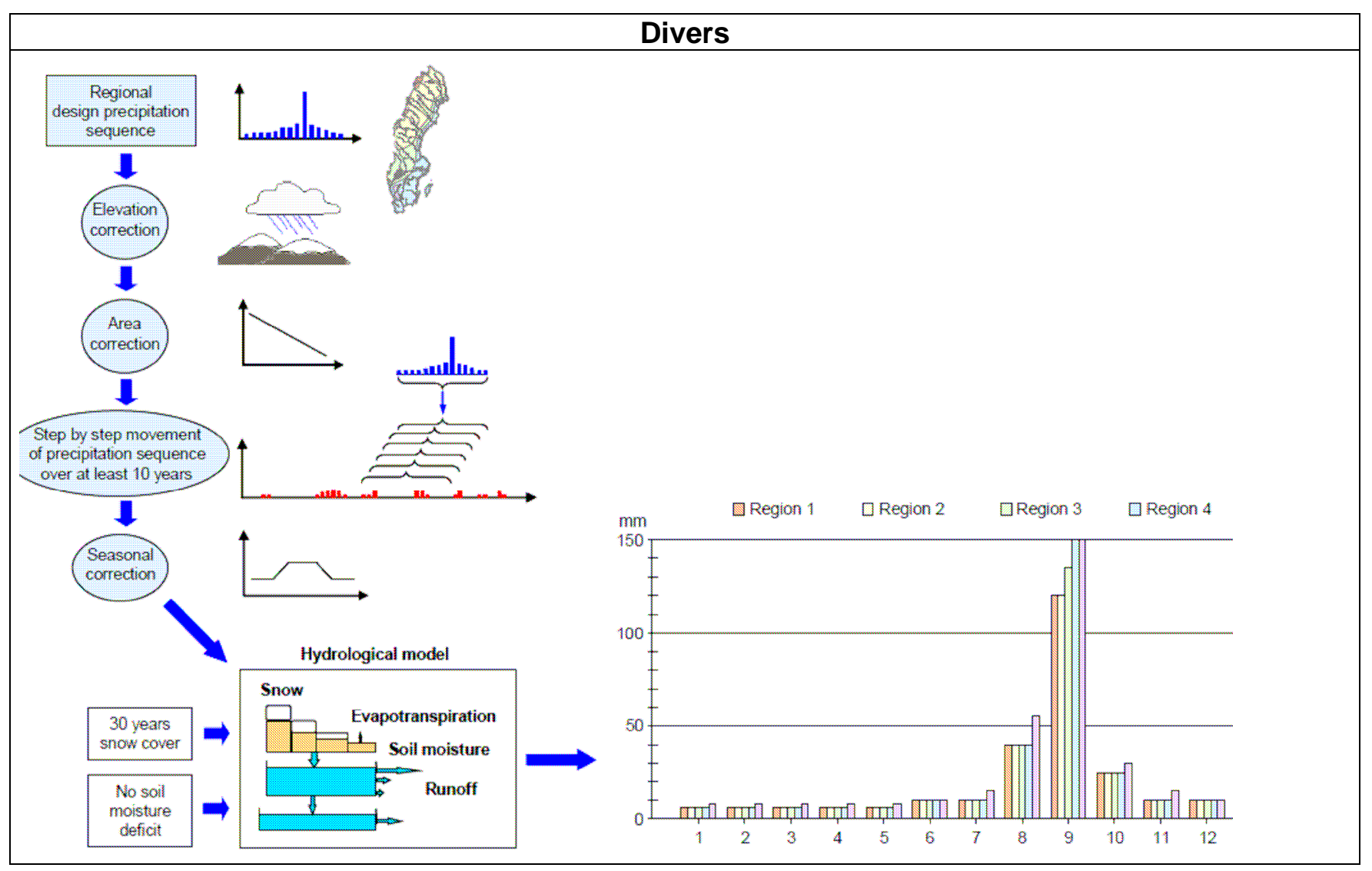




\section{Suisse}

Article G. Darbre lors du colloque Évacuateurs

Référence et date

Directive de 2008.

\section{Éléments de terminologie}

Crue de projet : événement exceptionnel ayant une période de retour de 1000 ans; hydrogramme QB $(\mathrm{t})$;

Crue de sécurité : événement extrême ; hydrogramme Qs(t).

Cote de danger: niveau du plan d'eau au-delà duquel la sécurité de l'ouvrage risque d'être compromise en raison d'un manque de stabilité ou de dégâts survenus ( $p$. ex. érosion du couronnement et des appuis ou affouillement au pied du barrage), d'une sous-pression accrue ou de l'érosion interne due à l'augmentation de la pression hydrostatique.

Revanche de sécurité : Cote de danger - Cote atteinte en crue de projet

\section{Classification des barrages}

\section{Critères de dimensionnement}

1 - La crue de projet doit être évacuée sans provoquer de dégâts, avec les critères suivants : revanche indicative

\begin{tabular}{|l|l|l|l|}
\hline Hauteur du barrage & $\mathrm{H}<10 \mathrm{~m}$ & $10 \mathrm{~m} \leq \mathrm{H}<40 \mathrm{~m}$ & $\mathrm{H} \geq 40 \mathrm{~m}$ \\
\hline Barrage en béton & $0,50 \mathrm{~m}$ & $1,00 \mathrm{~m}$ & $1,00 \mathrm{~m}$ \\
\hline $\begin{array}{l}\text { Barrage en remblai avec } \\
\text { protection pierreuse amont }\end{array}$ & $1,00 \mathrm{~m}$ & $2,00 \mathrm{~m}$ & $3,00 \mathrm{~m}$ \\
\cline { 2 - 4 } & $1,00 \mathrm{~m}$ & $1,50 \mathrm{~m}$ & $2,50 \mathrm{~m}$ \\
\hline
\end{tabular}

la vanne de plus forte débitance est hors service (règle «n-1 »)

les prises usinières sont fermées

l'éventualité d'une obstruction des organes de décharge doit être examinée.

2 - Sous crue de sûreté :

cote $\leq$ cote de danger

barrages en béton : toutes les vannes sont opérantes

barrages en remblai : règle « $n-1$ »

les prises usinières sont fermées.

3 - Cas particuliers :

stations de pompage-turbinage : combinaisons débits naturels - débits de pompage-turbinage

écrêteurs de crue : $Q_{B}$ : bassin plein et vidange $H S ; Q_{S}$ : bassin bas et vidange $H S$

ouvrages de montagnes : retenues pleines de sédiments et pertuis obstrués.

\section{Prise en compte de la disponibilité des évacuateurs}

Cote initiale : retenue normale.

Les phénomènes accompagnant un événement de crue doivent eux aussi être pris en compte: érosion, sédimentation, matériaux charriés, bois flottants, autres difficultés telles qu'interruption de courant ou accès

impossible suite à des conditions météorologiques extrêmes (orages, tempêtes).

\section{Méthodes de calcul de la crue}

Crue de projet :

- transformation pluie débit, en considérant plusieurs scénarios d'averses, de durée variable,

l'intégralité des pluies contribue aux apports

des formes d'hydrogrammes prédéfinies sont données (ambiguïté : il semble pourtant que le débit de pointe est déterminé indépendamment de cette forme spécifiée ?)

- il faut utiliser plusieurs méthodes pour calculer les crues.

Crue de sécurité :

choix 1:

- pour les ouvrages existants : $Q_{s}(t)=1.5{ }^{*} Q_{B}(t) \quad$ (débit et volume augmentés de $50 \%$ )

- pour les ouvrages neufs: Qs $(\mathrm{t})=1.5^{*} \mathrm{QB}_{\mathrm{B}}(2 / 3 \mathrm{t}$ ) (débit augmenté de $50 \%$ et volume de $\sim 100 \%$ )

choix 2 : 


\section{Divers}




\section{Annexe 2 : Calcul de la revanche due aux vagues}

\section{1 - Terminologie}

La revanche est la différence entre la cote de retenue et la crête du barrage. Cette définition tout à fait générale mérite deux commentaires :

- on sous-entend classiquement que la revanche est calculée pour les PHE. Or il arrive que des non spécialistes considèrent que la revanche est au-dessus de la $R N$. Confusion évidemment gênante. D'où la proposition de parler systématiquement désormais de revanche pour un état donné : revanche sur les PHE, sur la RN (cf. fig. A2.1). D'ailleurs, le principe, de plus en plus employé, consiste à calculer deux revanches, l'une sur RN, l'autre sur PHE puis à retenir le cas qui conduit à la cote « retenue + revanche » la plus élevée ;

- la parade pour assurer une protection contre les vagues peut consister en un mur pare-vagues placé sur le bord amont de la crête. Dans ce cas, la revanche est donnée par rapport au sommet du parapet (cf. fig. A2.2).

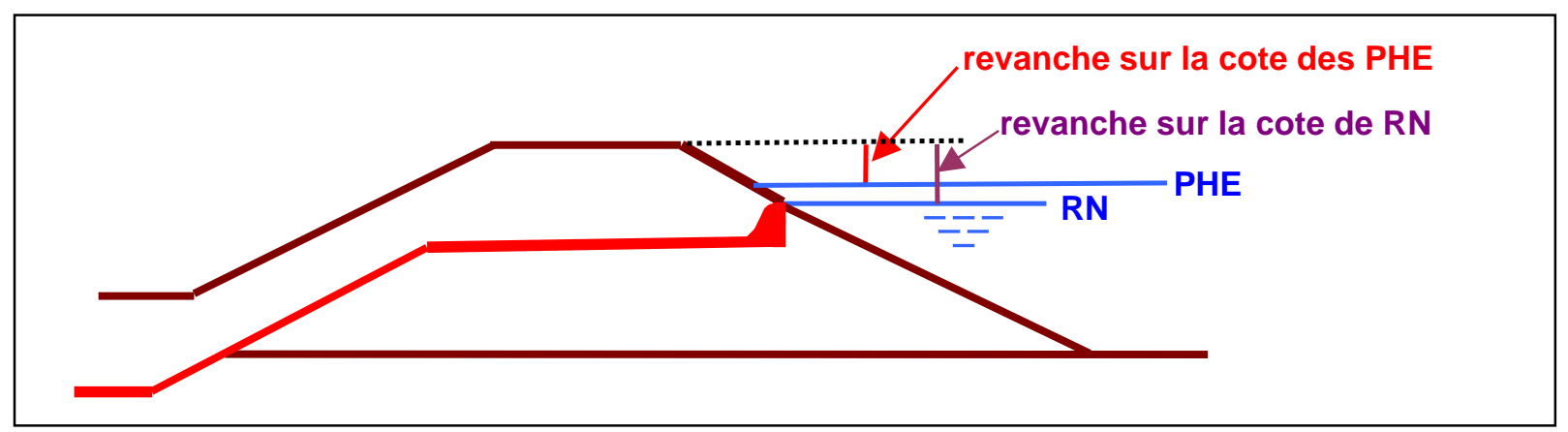

Figure A2.1 - Revanche sur les PHE ou sur la RN pour un barrage non équipé d'un mur pare-vagues

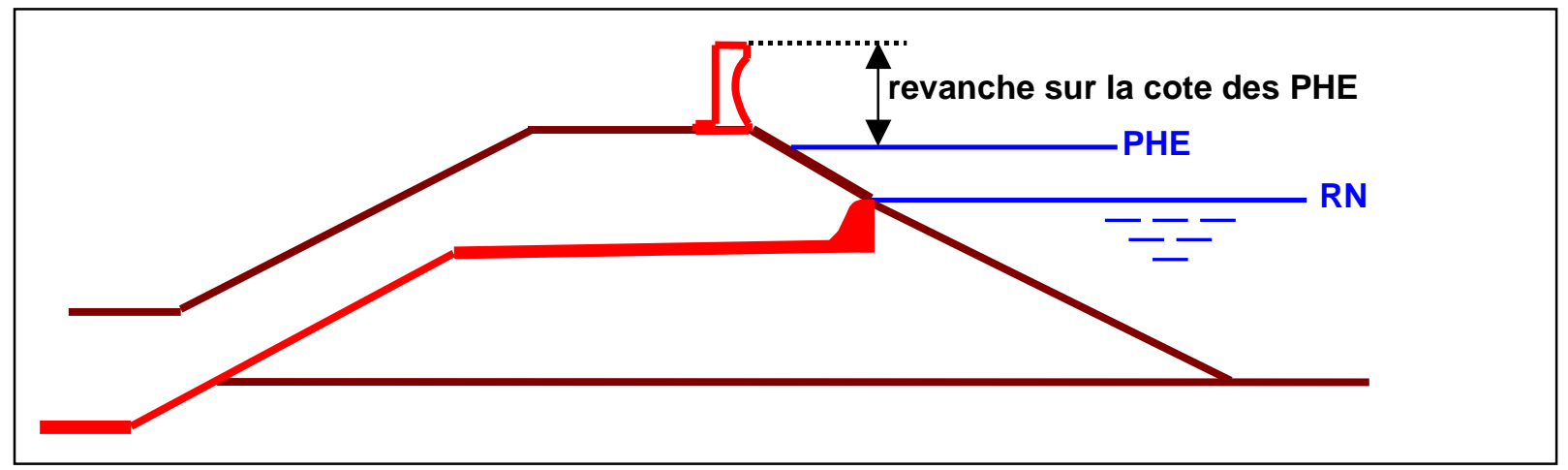

Figure A2.2 - Revanche sur les PHE pour un barrage équipé d'un mur pare-vagues

\section{2 - Déroulement du calcul}

Lorsque le vent souffle sur un plan d'eau, il génère au bout d'une certaine durée des vagues qui peuvent se propager en direction du barrage. En supposant que le vent souffle en direction du barrage, les vagues formées déferlent sur le parement. En fonction de la différence d'altitude existant entre la cote du plan d'eau en situation de vent de projet et la crête, un certain pourcentage des vagues passe par-dessus la crête et ruisselle sur le parement aval. Dans le cas d'un barrage en remblai, une érosion plus ou moins importante de la crête et du parement aval en résulte. Un barrage en béton ou en maçonnerie sans 
remblai à l'aval résiste à l'action des vagues ; mais pour des raisons liées à l'exploitation, il n'est pas souhaitable que la crête soit le siège de déferlements fréquents. On doit donc positionner la crête à une altitude suffisante pour que le pourcentage de vagues qui l'atteignent soit faible.

On considère généralement deux situations de projet vis-à-vis du vent :

- un vent de période de retour 50 ans soufflant sur une retenue qui se trouve à la cote des PHE ; c'est cette situation qui s'avère généralement dimensionnante, sauf pour les retenues où $\mathrm{RN}=\mathrm{PHE}$;

- un vent de période de retour 1000 ans sur la retenue normale.

Pour chaque situation de projet vis-à-vis du vent, le calcul de la revanche s'effectue ensuite en quatre étapes ( $\$ 4$ à 7 ).

\section{3 - Le vent}

Pour la connaissance du vent, il faut distinguer :

- une approche avant-projet, où on peut se contenter d'une carte de vent, par exemple ROSA 2000 (CETMEF, 2001) ou règles NV 65 (CSTB, 2009) ;

- une approche projet, où il est nécessaire d'obtenir de Météo France des données plus locales, avec indication des vents selon leur direction.

On a vu que la revanche doit être calculée dans les deux combinaisons rares suivantes : à partir de la cote des PHE avec un vent de période de retour 50 ans et aussi à partir de la RN avec un vent de période de retour 1000 ans. En l'absence de données de vent pour des périodes de retour élevées, on pourra utiliser les indications de ROSA 2000 :

La vitesse de vent pour une période de retour $T$ en années est donnée par :

$$
\frac{V_{T}}{V_{50}}=\sqrt{\frac{1-0,33 \cdot \ln (-\ln (1-1 / T))}{2,29}} \text { ou par le tableau A2.1 suivant : }
$$

\begin{tabular}{|c|c|}
\hline $\begin{array}{c}\text { Période de retour } T \\
\text { (ans) }\end{array}$ & $\begin{array}{c}\text { Facteur } \\
\text { multiplicatif }\end{array}$ \\
\hline 5 & 0,81 \\
\hline 10 & 0,87 \\
\hline 20 & 0,93 \\
\hline 50 & 1 \\
\hline 100 & 1,05 \\
\hline 200 & 1,10 \\
\hline 500 & 1,15 \\
\hline 1000 & 1,20 \\
\hline 10000 & 1,33 \\
\hline
\end{tabular}

Tableau A2.1 - Facteur multiplicatif entre la vitesse du vent 50 ans et la vitesse du vent $T$ ans

On retiendra que la vitesse du vent pour une période de retour 1000 ans vaut 1,2 fois celle pour 50 ans. Par exemple, si la vitesse de vent cinquantennale vaut $150 \mathrm{~km} / \mathrm{h}$, la vitesse millennale vaut $180 \mathrm{~km} / \mathrm{h}$. 


\section{4 - Estimation de la hauteur des vagues sur le plan d'eau}

Connaissant la vitesse du vent (cf. § 3), on estime la hauteur des vagues ; plutôt qu'à la hauteur maximale des vagues, on s'intéresse à leur hauteur significative (notée $H_{s}$ ) c'est-àdire la hauteur moyenne du tiers supérieur de toutes les vagues du spectre.

Pour le calcul de la hauteur des vagues, on propose d'utiliser la formule de (Smith et al., 1991), adaptée aux conditions d'eau profonde :

$H_{S}=0,00048 . \hat{U}_{a} \sqrt{F}$

$H_{s}$ désigne la hauteur significative des vagues en $m$;

$F$ est la longueur du fetch ${ }^{16}$ de la retenue en $\mathrm{m}$ (cf. fig. A2.3) ;

$\hat{U}_{a}$ désigne la vitesse efficace ajustée du vent à $10 \mathrm{~m}$ de hauteur en $\mathrm{m} / \mathrm{s}$, soit $\hat{U}_{a}=U_{a} \cdot \cos \theta$ avec $\theta$ l'angle entre les directions du vent et des vagues ;

$U_{a}=U \sqrt{(0,75+0,067 \times U)}$

avec $U$ vitesse moyenne du vent sur la durée à considérer, à $10 \mathrm{~m}$ au-dessus de la surface de l'eau, pour la période de retour choisie. Sans information sur la direction des vents, on prendra $\hat{U}_{a}=U_{a}$.

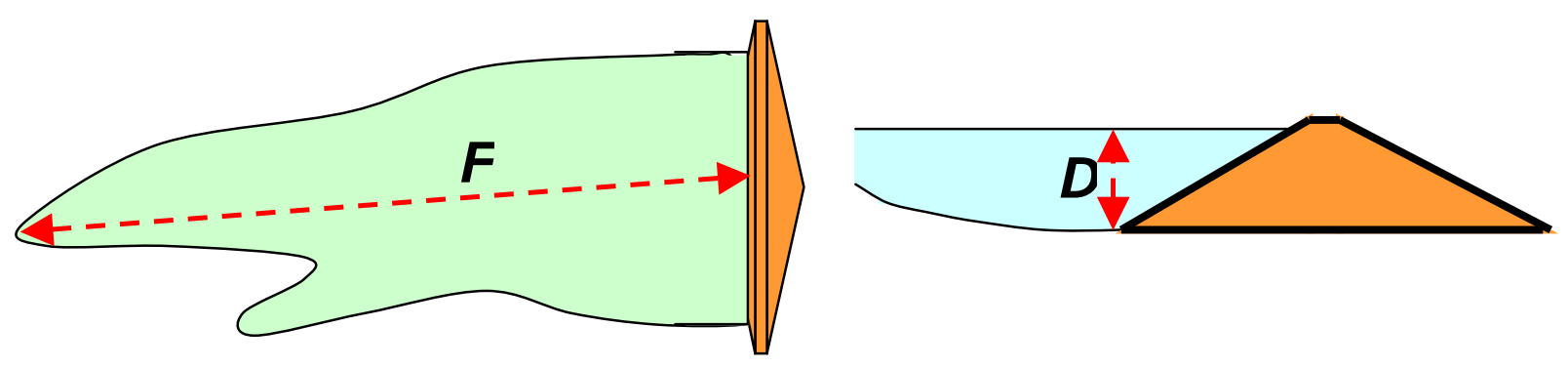

Figure A2.3 - Paramètres intervenant dans le calcul des vagues, pour une situation de projet donnée

Dans le cas des vents supérieurs à $120 \mathrm{~km} / \mathrm{h}$, cette méthode donne de meilleurs résultats que celle de Bretschneider, dont les formules font également intervenir la profondeur $D \mathrm{du}$ plan d'eau (cf. fig. A2.3), et qui est proposée dans l'ouvrage (CFBR, 1997).

Dans cette formule (2), il faut donner la vitesse du vent à la surface de l'eau. Comme les données proviennent en général d'une station terrestre, elles doivent alors être corrigées du fait du passage du vent sur la surface très lisse du plan d'eau (majoration de 1,01 pour un fetch de $100 \mathrm{~m}$, de 1,06 pour un fetch de $500 \mathrm{~m}$, de 1,1 pour un fetch de $1000 \mathrm{~m}$, de 1,2 pour un fetch de $5000 \mathrm{~m}$ et de 1,3 à partir de $8 \mathrm{~km}$ selon (Saville et al., 1962)).

Une seconde correction sur les données de vitesse de vent (généralement sur une heure) est nécessaire pour les transposer à la durée minimale de vent $\left(t_{\min }\right)$ nécessaire à la formation des vagues. C'est la durée à partir de laquelle les hauteurs de vague sont limitées par le fetch et elle correspond aux vagues de hauteur maximale. Elle peut être obtenue par application d'une autre formule de (Smith et al., 1991) :

$t_{\min }=27 . F^{0,72} \cdot \hat{U}_{a}^{-0,44}$

\footnotetext{
${ }^{16}$ On appelle « fetch » la surface d'eau continue sur laquelle le vent souffle.
} 
La vitesse moyenne du vent sur une heure $\left(U_{3600}\right)$ est liée à la vitesse $U_{t_{\min }}$ correspondant à $t_{\min }$ par la formule suivante, selon (USACE, 1984) :

si $\mathrm{t}_{\min }<3600 \mathrm{~s}, \quad \frac{U_{t_{\min }}}{U_{3600}}=1,277+0,296 \cdot \tanh \left(0,9 . \log \frac{45}{t_{\text {min }}}\right)$

et si $t_{\min }>3600 \mathrm{~s} \quad \frac{U_{t_{\min }}}{U_{3600}}=1,5334-0,15 \cdot \log \left(t_{\min }\right)$

Un calcul itératif permet de déterminer $U_{a}$ correspondant à la donnée du vent mesurée au sol pour la durée adéquate $\mathrm{t}_{\min }{ }^{17}$.

Le tableau A2.2 ci-après donne les valeurs de la hauteur significative des vagues pour des cas courants (profondeur de l'eau $D$ et longueur du fetch $F$ en mètres), calculée par la méthode de Smith pour les cas d'eau profonde. Elles ne dépendent pas de la profondeur d'eau. Pour $D=5 \mathrm{~m}$, les valeurs résultent de l'application de formules valables en eau non profonde, dues à Bretschneider et Reid (USACE, 1984) et sont légèrement inférieures, d'au plus $6 \%$ dans les exemples du tableau.

\begin{tabular}{|c|c|c|c|c|c|c|c|}
\hline & $\mathrm{U}$ & \multicolumn{2}{|c|}{$120 \mathrm{~km} / \mathrm{h}$} & \multicolumn{2}{c|}{$150 \mathrm{~km} / \mathrm{h}$} & \multicolumn{2}{c|}{$180 \mathrm{~km} / \mathrm{h}$} \\
\hline $\mathrm{D}(\mathrm{m}) \downarrow$ & $\mathrm{F}(\mathrm{m})$ & 100 & 500 & 100 & 500 & 100 & 500 \\
\hline 5 & & 0,27 & 0,59 & 0,49 & 0,98 & 0,64 & 1,26 \\
\hline 10 & & 0,28 & 0,61 & 0,49 & 1,02 & 0,64 & 1,34 \\
\hline 15 & & 0,28 & 0,61 & 0,49 & 1,02 & 0,64 & 1,34 \\
\hline
\end{tabular}

Tableau A2.2 - Hauteur significative des vagues $\left(H_{s}\right)$ en fonction de la vitesse du vent $(U)$, de la longueur du fetch de la retenue $(F)$ et de sa profondeur $(D)$

\section{5 - Calcul de la vague de projet}

On calcule ensuite une vague de projet (notée $H_{d}$ ) qui dépend du pourcentage de vagues du spectre que l'on accepte de laisser dépasser la crête. Elle est classiquement exprimée sous la forme $H_{d}=K . H_{s}$, où le coefficient $K$ prend les valeurs suivantes, selon (Saville et al., 1962) :

\begin{tabular}{|c|c|}
\hline $\mathrm{K}$ & $\begin{array}{c}\text { \% de vagues } \\
\text { dont la hauteur dépasse } H_{d}\end{array}$ \\
\hline 0,75 & $32 \%$ \\
\hline 0,89 & $20 \%$ \\
\hline 1,00 & $13 \%$ \\
\hline 1,07 & $10 \%$ \\
\hline 1,25 & $5 \%$ \\
\hline 1,33 & $3 \%$ \\
\hline 1,58 & $1 \%$ \\
\hline
\end{tabular}

Tableau A2.3 - Coefficient K utilisé pour le calcul de la vague de projet

\footnotetext{
${ }^{17}$ Pour la première itération, on part de $U=U_{3600}$ corrigé de l'effet plan d'eau lisse, on calcule $U_{a}$ par (2) puis $t_{\min }$ par (3). La $2^{\text {ème }}$ itération consiste à recalculer $U$ correspondant au $t_{\min }$ précédemment calculé par la formule (4) puis $U_{a}$ par (2), puis $t_{\min }$ correspondant à ce nouveau $U_{a}$ par (3). Et ainsi de suite. On s'arrête lorsque $t_{\min }$ est stabilisé et 3 ou 4 itérations suffisent. Les tableaux fournis dispenseront en général de faire ces calculs dans des cas courants.
} 
Le pourcentage de vagues que l'on autorise à passer sur l'ouvrage dépend de la résistance des matériaux à l'impact des vagues.

Il est recommandé d'accepter que $5 \%$ des vagues déferlent $(K=1,25)$ pour les barrages en terre, $13 \%$ pour les barrages à parement aval en enrochements $(K=1,0)$ et $30 \%$ pour les barrages-poids ou voûtes ( $K=0,75$ ). Toutefois, on adoptera la même valeur que pour les barrages en remblai ( $K=1,25)$ dans les cas suivants :

- barrage poids fondé sur terrain meuble ou érodable, cas a priori rare et limité à de très petits ouvrages ;

- barrage-poids conforté par un remblai aval ;

- barrage-poids ou voûte comportant une plate-forme aval en remblai avec des dispositifs d'auscultation, ou des voies d'accès à divers organes importants du barrage...

Le cas des barrages mobiles au fil de l'eau, pour lesquels la sensibilité au déversement est faible, mérite une détermination au cas par cas.

\section{6 - Détermination de la hauteur de déferlement}

Pour la vague de projet, on estime la hauteur de déferlement (run-up en anglais), notée $R$, qui est la différence de hauteur verticale entre le niveau maximal atteint par le déferlement de la vague sur le parement amont et le niveau du plan d'eau ; cette hauteur dépend de la nature du barrage (la pente du parement, la rugosité du matériau), comme l'illustre la figure A2.4 ci-dessous :

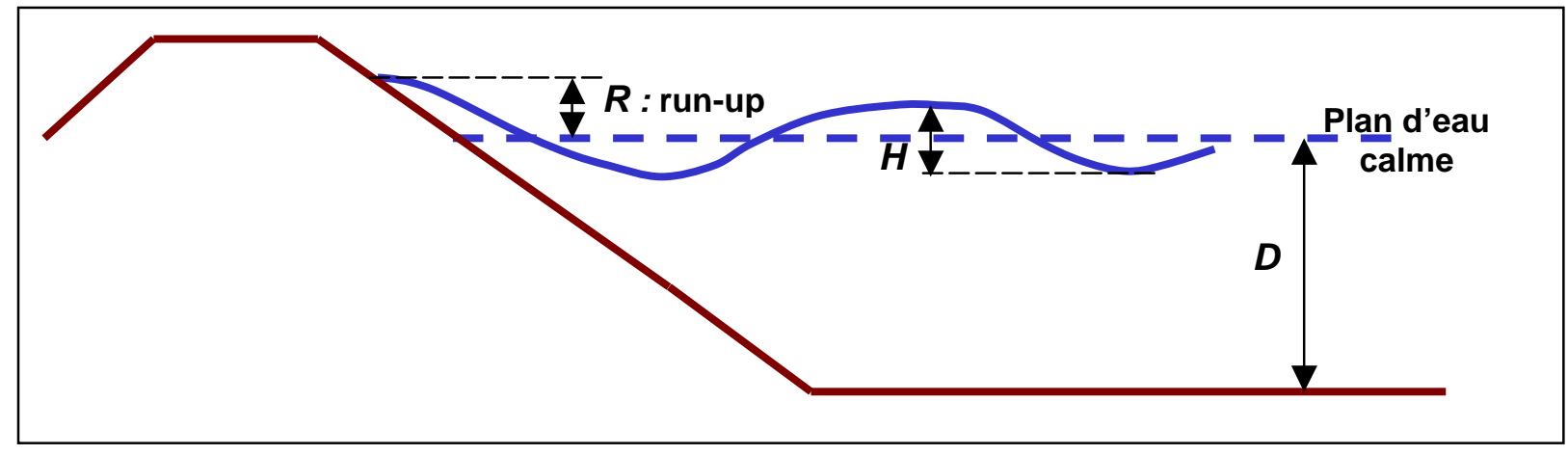

Figure A2.4 - Run-up dans le cas d'une onde sinusoïdale (cas du vent)

Le premier cas est celui d'un barrage en remblai à parement amont lisse (masque en béton, géomembrane non recouverte). Le déferlement relatif de la vague $(R / H d)$ est donné par le tableau A2.4 résultant de la formule de (Saville et al., 1962), en fonction de la pente du talus et de $H_{d} L ; L$, longueur d'onde des vagues, est donnée par:

$L=1,56 . T^{2}$, formule valable si $D>L / 2 . T$ est la période du pic spectral des vagues et peut être estimée, par une formule due à (Smith, 1991) :

$T=0,0716 \cdot F t^{0,28} \cdot \hat{U}_{a}^{0,44}$

\begin{tabular}{|c|c|c|c|}
\hline Pente du talus amont $\rightarrow$ & $1 / 3$ & $1 / 2,5$ & $1 / 2$ \\
\hline$H_{d} / L=0,1$ & 1,15 & 1,4 & 1,9 \\
\hline$H_{d} / L=0,08$ & 1,37 & 1,64 & 2,0 \\
\hline$H_{d} / L=0,07$ & 1,49 & 1,73 & 2,0 \\
\hline
\end{tabular}

Tableau A2.4 - Valeurs du déferlement relatif $R / H_{d}$ sur un parement lisse 
Ces résultats conduisent aux valeurs de revanche du tableau A2.5, pour $K=1,25$ :

\begin{tabular}{|c|c|c|c|c|c|c|c|}
\hline & $U \rightarrow$ & \multicolumn{2}{|c|}{$120 \mathrm{~km} / \mathrm{h}$} & \multicolumn{2}{c|}{$150 \mathrm{~km} / \mathrm{h}$} & \multicolumn{2}{c|}{$180 \mathrm{~km} / \mathrm{h}$} \\
\hline$D \downarrow$ & $F \rightarrow$ & $100 \mathrm{~m}$ & $500 \mathrm{~m}$ & $100 \mathrm{~m}$ & $500 \mathrm{~m}$ & $100 \mathrm{~m}$ & $500 \mathrm{~m}$ \\
\hline $5 \mathrm{~m}$ & & 0,53 & 1,13 & 0,71 & 1,48 & 0,92 & 1,85 \\
\hline $10 \mathrm{~m}$ & & 0,53 & 1,19 & 0,71 & 1,61 & 0,92 & 2,06 \\
\hline $15 \mathrm{~m}$ & & 0,53 & 1,19 & 0,71 & 1,63 & 0,92 & 2,06 \\
\hline
\end{tabular}

Tableau A2.5 - Hauteur du déferlement $R$ pour un parement amont lisse de pente 1/3

Le second cas, est celui d'un barrage en remblai à parement amont rugueux. Les vagues déferlent alors moins haut. On propose, selon les publications de l'Institution of Civil Engineers (ICE, 1996) et le South African Committee on Large Dams (SACOLD, 1990), de prendre $60 \%$ de la revanche nécessaire pour un parement amont lisse. D'où le tableau A2.6 construit à partir du tableau A2.5, toujours pour $K=1,25$ :

\begin{tabular}{|c|c|c|c|c|c|c|c|}
\hline & $U \rightarrow$ & \multicolumn{2}{|c|}{$120 \mathrm{~km} / \mathrm{h}$} & \multicolumn{2}{c|}{$150 \mathrm{~km} / \mathrm{h}$} & \multicolumn{2}{c|}{$180 \mathrm{~km} / \mathrm{h}$} \\
\hline$D \downarrow$ & $F \rightarrow$ & $100 \mathrm{~m}$ & $500 \mathrm{~m}$ & $100 \mathrm{~m}$ & $500 \mathrm{~m}$ & $100 \mathrm{~m}$ & $500 \mathrm{~m}$ \\
\hline $5 \mathrm{~m}$ & & 0,32 & 0,68 & 0,43 & 0,89 & 0,55 & 1,11 \\
\hline $10 \mathrm{~m}$ & & 0,32 & 0,72 & 0,43 & 0,96 & 0,55 & 1,23 \\
\hline $15 \mathrm{~m}$ & & 0,32 & 0,72 & 0,43 & 0,98 & 0,55 & 1,23 \\
\hline
\end{tabular}

Tableau A2.6 - Hauteur du déferlement $R$ pour un parement amont rugueux de pente 1/3

Quelle que soit la rugosité du parement amont, pour une pente de $1 / 2,5$, les valeurs de déferlement obtenues pour une pente de $1 / 3$ sont à multiplier par 1,2 . Pour une pente de $1 / 2$, elles sont à multiplier par 1,4 .

Le troisième cas est celui d'un barrage en maçonnerie ou en béton à parement amont vertical ou quasiment vertical. Selon (ICE, 1989), la valeur du run-up est de l'ordre de grandeur de la hauteur de vague en eau profonde. On adoptera donc les valeurs du tableau A2.2 pour la hauteur caractéristique, à affecter du coefficient 0,75 ou 1,25 pour la vague de projet $(0,75$ pour le cas général ; 1,25 si une partie meuble aval joue un rôle important pour la stabilité ou pour l'exploitation).

\section{7 - Détermination de la cote de la crête du barrage ou du pare-vague}

À l'issue du calcul de hauteur de déferlement pour les deux situations de projet, on trouve la plus forte des cotes $R N+R_{R N}$ et $P H E+R_{P H E}$ et on fixe la cote de la crête (après tassement) au moins à cette valeur ; en variante, on peut atteindre cette cote à l'aide d'un mur pare-vagues construit contre le bord amont de la crête. Dans ce cas, la cote de crête doit être au moins égale à celle des PHE. Dit autrement, le pare-vagues sert uniquement aux vagues, pas à protéger le barrage contre la crue de projet. II peut jouer le rôle de parapet pour la sécurité des personnels ou des automobilistes si une route publique emprunte la crête. La largeur de la crête doit éventuellement être adaptée à la présence d'un parevagues pour disposer d'une piste de circulation de largeur suffisante. II est recommandé que la hauteur du pare-vagues ne dépasse pas $1,5 \mathrm{~m}$.

Nous constatons que la revanche nécessaire pour les vagues varie dans une large gamme en fonction des conditions de vent, de la longueur de la retenue, de la pente du talus amont et de sa rugosité. Des règles forfaitaires simplistes ne peuvent être suffisantes et un calcul est indispensable à chaque fois. Le cas d'un parement lisse de barrage en remblai est nettement le plus défavorable. 


\section{Annexe 3: Quelques exemples d'adaptation d'évacuateurs de crues de barrages existants}

Dans le cas de barrages existants, le passage de crues majorées par rapport à celle utilisée pour le dimensionnement peut imposer une majoration de la débitance des évacuateurs. Dans de nombreux cas, cette majoration peut-être obtenue par une optimisation du seuil et/ou une élévation de la cote de la retenue.

Dans ce deuxième cas en particulier, l'évacuateur risque de fonctionner dans un domaine non prévu à la conception. Or les évacuateurs ont été optimisés pour la PHE lors de la construction, pour une crue de type exceptionnelle, en général sur modèle réduit pour les grands barrages. Ce qui se passe au-delà n'a a priori pas été regardé d'un point de vue hydraulique et génie civil et ces points devront faire l'objet de vérifications attentives.

L'élévation de la cote de retenue pose des problèmes spécifiques à chaque ouvrage. La présente annexe en donne quelques exemples. La plupart du temps, des essais sur modèles physiques sont manifestement nécessaires pour remettre à niveau les ouvrages et doivent explorer des gammes de crues jusqu'à la crue extrême.

\section{1 - Évacuateur libre en crête}
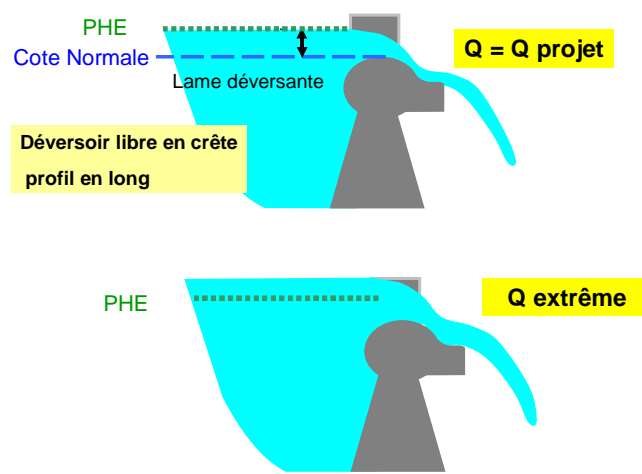

Figure A3.1 - Évacuateur libre, crue extrême, vue amont-aval

Pour un évacuateur libre, le passage de la crue exceptionnelle à la crue extrême ne pose a priori pas de problème de géométrie, seule la cote des bajoyers est à rectifier (cf. fig. A3.1 et A3.2).

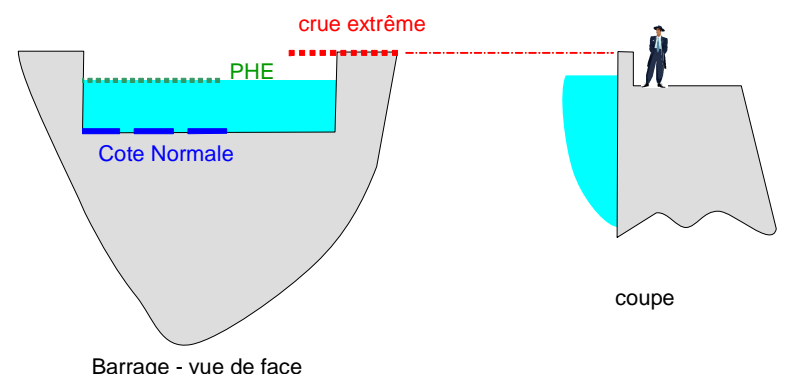

Figure A3.2 - Évacuateur libre, crue extrême, vue de face

Des problèmes hydrauliques peuvent apparaître (passage en dépression de la lame déversante en crête par exemple). 


\section{2 - Pont en crête}

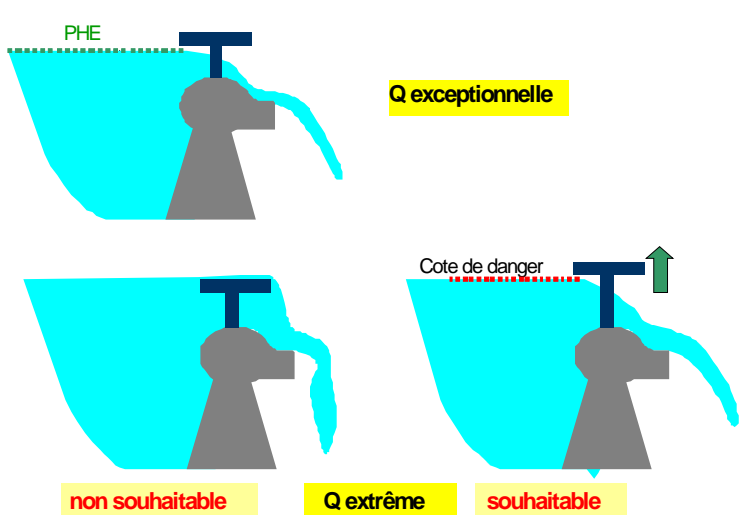

Figure A3.3 - Évacuateur libre, crue extrême, avec pont en crête

Si un pont surplombe l'évacuateur, il pourra être accroché par des crues supérieures à la crue de dimensionnement, avec des conséquences néfastes: accès impossible aux installations, destruction du pont, etc. Il devra donc être surélevé (cf. fig. A3.3).

\section{3 - Évacuateur vanné en crête à surface libre}

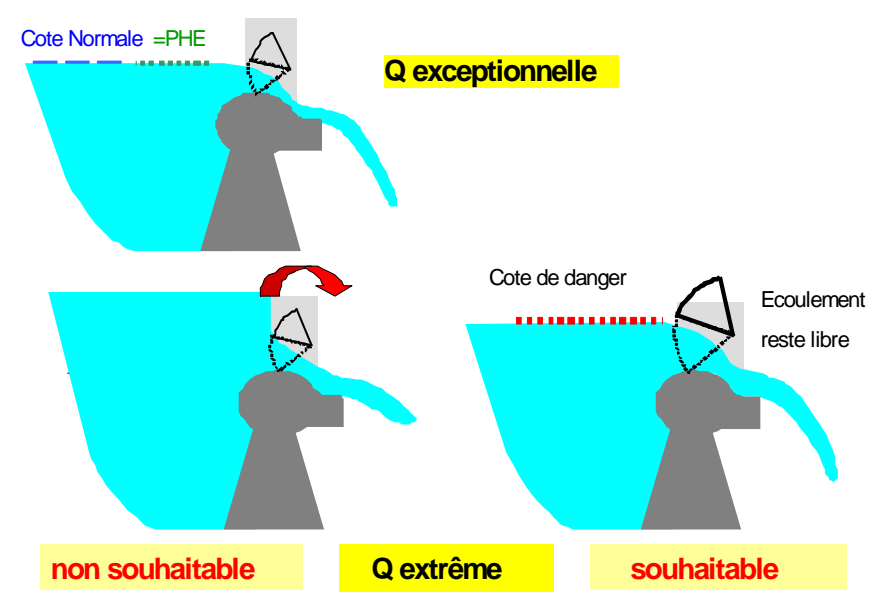

Figure A3.4 - Évacuateur vanné libre, crue extrême

Pour la crue exceptionnelle, vannes ouvertes, l'écoulement sur l'évacuateur est à surface libre. Pour la crue extrême, il risque de passer en charge, avec risque de submersion des superstructures (cf. fig. A3.4).

Revenir à la solution hydrauliquement souhaitable (cf. fig. A3.4 - schéma de droite) remet en cause tout le génie civil. II faut trouver une autre solution : rendre la submersion acceptable, évacuateur supplémentaire... 


\section{4 - Évacuateur vanné en crête en charge}
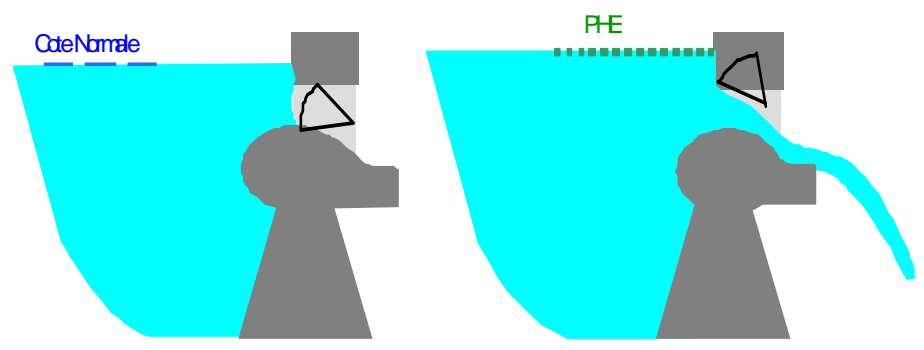

\section{Qexoeptionnelle}

Figure A3.5 - Évacuateur en charge, crue exceptionnelle

Pour la crue exceptionnelle, l'écoulement vannes ouvertes reste en charge, les PHE pouvant rester égales à la cote normale (cf. fig. A3.5).

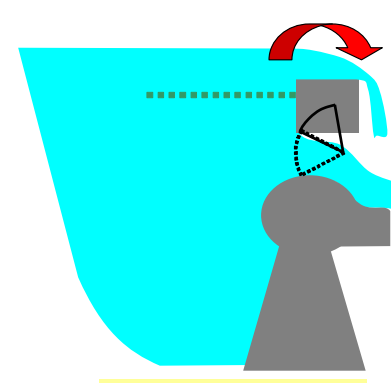

non souhaitable

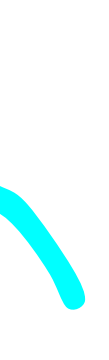

Q extrême

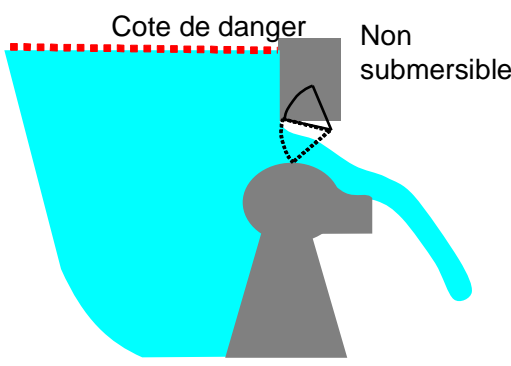

souhaitable

Figure A3.6 - Évacuateur en charge, crue extrême

Pour la crue extrême (cf. fig. A3.6), le niveau de la retenue va monter notablement, car l'écoulement en charge est peu sensible à l'augmentation de niveau de la retenue. La solution souhaitable peut demander une surélévation notable des superstructures, la poussée sur les vannes augmentant alors de manière importante.

\section{5 - Barrage annexe, crue extrême}

Ce cas a été abordé dans les recommandations pour les barrages multiples (cf. 4.3.3.3). 

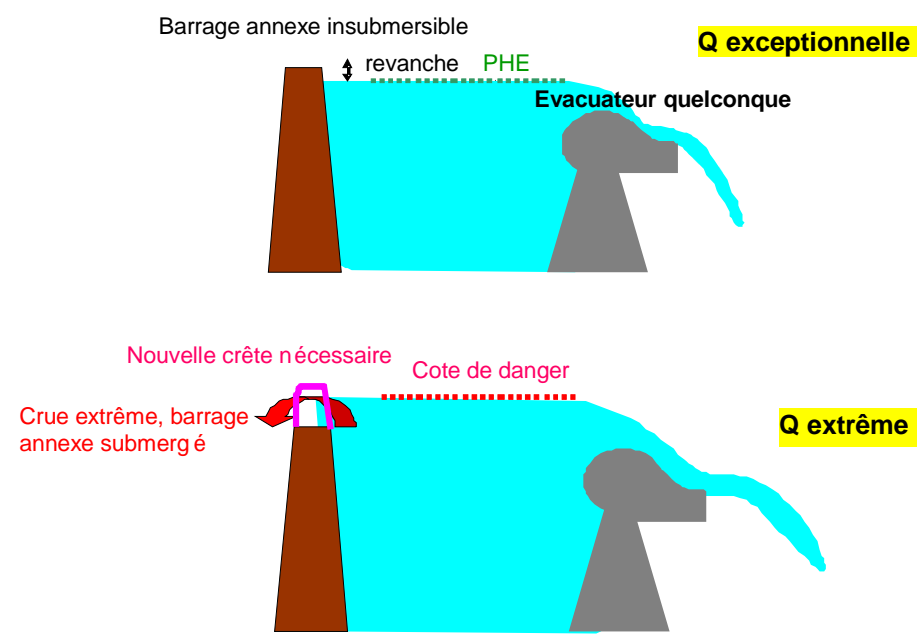

Figure A3.7 - Barrage annexe, crue extrême

Pour la crue exceptionnelle, le barrage annexe insubmersible est calé à PHE + revanche.

Pour la crue extrême, ce barrage peut devenir submersible, avec risque de rupture. II convient donc de rehausser la cote de ce barrage à la cote atteinte par la crue extrême et même au-delà (cf. fig. A3.7).

\section{6 - Coursier aval}

En cas de coursier aval, celui-ci a été dimensionné à la conception pour une crue de type exceptionnelle.

Pour la crue extrême, des problèmes peuvent survenir, tels que :

- débordement latéral ;

- instabilité éventuelle de l'écoulement ;

- surpression dans les courbes concaves (avec traction sur le béton) ;

- surcharge sur le béton (cuillère du saut de ski final) ;

- noyage de la cuillère, auparavant dénoyée ;

- etc. 


\section{Annexe 4 : Bibliographie}

\section{Réglementation - Recommandations :}

- Australie - Australian dam safety committee guidelines DSC 13 et DSC 11;

- Badin B., Chatenoux O., Cochet P., Foucault C., Sabaton A. - Risques liés aux équipements hydromécaniques des ouvrages évacuateurs de crues des barrages -23 ème congrès de la CIGB, Q.91, R.37 - 2009 ;

- Bister D., coordinateur - Recommandations pratiques pour améliorer la sécurité des barrages en crue. Practical guidelines for improvement of dam safety during floods CFGB, Revue Barrages et Réservoirs n̊8 - 1998 ;

- Bister D., Lempérière F., Le Delliou P., Marchesini P. - Évaluation et amélioration de la sécurité vis-à-vis des crues $-18^{\text {ème }}$ congrès de la CIGB, Q.68, R.41 - 1994 ;

- Brésil - Criterios de projeto civil de usinas hidrelectricas - Octobre 2003 ;

- Bureau d'Étude Technique et de Contrôle des Grands Barrages (BETCGB) Ministère de l'Économie, des Finances et de l'Industrie - Guide pour le contrôle des barrages en exploitation - 2002 ;

- Centre d'Études Techniques Maritimes Et Fluviales (CETMEF) - ROSA 2000 Recommandations pour le calcul aux états limites des ouvrages en site aquatique 2001 ; presses ENPC ; http://www.cetmef.developpement-durable.gouv.fr/rosa-2000application-des-r54.html

- Comité Français des Barrages et Réservoirs (CFBR) - Recommandations pour la justification de la stabilité des barrages et des digues en remblai - Recommandations provisoires -2010 ;

- Comité Français des Barrages et Réservoirs (CFBR) - Recommandations pour la justification de la stabilité des barrages-poids - 2012 ;

- Comité Français des Grands Barrages (CFGB) - Petits Barrages, recommandations pour la conception, la réalisation et le suivi - Cemagref édition - 1997 ; http://www.barrages-cfbr.eu

- Comité Français des Grands Barrages (CFGB) - Barrages et Réservoirs næ8 Recommandations pratiques pour améliorer la sécurité des barrages en crue - 1998 ;

- Décret 2007-1735 du 11 décembre 2007 relatif à la sécurité des ouvrages hydrauliques ;

- Électricité de France - Document interne EDF-DPIH - Barrages équipés de vannes d'évacuation des crues : conception, exploitation et maintenance des organes de crue, de leurs commandes et des automatismes associés - 2009 ;

- Eurocode 1 - Bases de calcul et actions sur les structures - 2003 \& ultérieur (voir http://www.afnor.org/profils/activite/construction/les-eurocodes/les-eurocodes)

- Eurocode 1 : actions sur les structures - Partie 1-4 : actions générales - Actions du vent (NF EN 1991-1-4 Novembre 2005) 
- Ginocchio R. - L'énergie hydraulique - Eyrolles - 1978 ;

- Italie - Loi italienne de 1959 ;

- Italie - « règles techniques » de 1982 ;

- Italie - circulaire de 2004 ;

- Naudascher E. - Hydrodynamic forces - Design manual - 1991;

- Norvège - Norwegian dam safety regulation - Water ressources act - Janvier 2010 ;

- Québec - Loi sur la sécurité des barrages - 2002 ;

- Québec - Décret D. 300-2002, 2002, G.O 2, 2043 ;

- Royaume-Uni - United Kingdom reservoirs act - 1975 ;

- Royaume-Uni - United Kingdom - Guide floods and reservoir safety - 1978 (mis à jour en 1989 et 1996) ;

- Service Technique Central des Ports Maritimes et Voies Navigables (STCPMVN) Notice STC QC N93.01 - Programme LUBAQUA 1993 ;

- Suède - Swedish guidelines for design flood determination for dams - 2007 ;

- Suisse - Directive suisse de 2008 ;

- USA - Federal Emergency Management Agency (FEMA) - Federal guidelines for dam safety - Selecting and accommodating inflow design floods for dams - 1998 (reprinted 2004);

- US Bureau of Reclamation - Design of small dams - US Government printing office, third edition - 1987 ;

- Varlet H. - Usines de retenue - Usines de plaine - Eyrolles - 1962 ;

Dimensionnement des éléments mécaniques:

- DIN19.704 1,2 et 3 - Bases de calcul pour constructions hydrauliques en acier - 1998 ;

- Eurocode 3 - Partie 1.1 - Calcul des constructions en acier - 2005 et ultérieur;

- Fédération Européenne de la Manutention (FEM) - Règles pour le calcul des appareils de levage $-3^{\text {ème }}$ édition révisée -1998 ;

- Parlement Européen et Conseil du 22 juin 1998 - Directive 98/37/CE, Rapprochement des législations des États membres relatives aux machines - 1998 ;

Hydrologie : 
- Arnaud P., Lavabre J. - La modélisation stochastique des pluies horaires et leur transformation en débits pour la prédétermination des crues - Revue des Sciences de l'Eau 13/4 - 2000 ;

- Arnaud P., Lavabre J. - Coupled rainfall model and discharge model for flood frequency estimation - Water Resources Research - vol. 38, nº - 2002 ;

- Benito, G. , Lang, M., Barriendos, M., Llasat, M.C., Frances, F., Ouarda, T., Thorndycraft, V., Enzel Y., Bardossy A., Coeur, D., Bobee, B. - Use of Systematic, Paleoflood and Historical data for the improvement of flood risk estimation - Review of scientific methods - Natural Hazard, Special number "Strategies and Applications in Natural Hazard Research using Historical Data" - Edited by T. Glade \& M. Lang, Kluwer Academic Publishers - 2004 ;

- Bowles D. S. - Risk perception and acceptance criteria : with applications to dams Risk Management In Civil Engineering Advanced Course - 17-21 Novembre 2008 ;

- Carvajal C., Peyras L., Arnaud P., Boissier D., Royet P. - Probabilistic Modelling of Flood Water Level for Dam Reservoirs - ASCE Journal of Hydrologic Engineering Vol. 14 Issue $3-2009$;

- Cayla 0. - Synthèse régionale pluviométrique en région montagneuse - Lausanne Symposium Hydrology in Mountainous Regions - IAHS Publ. n993 - 1990 ;

- Cayla O. - Probabilistic calculation of design floods: SPEED - San Francisco International Symposium on Engineering Hydrology - American Society of Civil Engineers - 1995 ;

- Cernesson F., Lavabre J., Masson J.M. - Stochastic model for generating hourly hyetographs - Atmospheric Research - vol. 42 nㅇ-4 - 1996 ;

- Champion M. - Les inondations en France du VI ${ }^{\text {ème }}$ siècle au XIXème siècle - Éditions Quae - réédition 2002 (cédérom) ;

- Chave S. - Pertinence de la cartographie hydrogéomorphologique dans l'approche des inondations rares à exceptionnelles: exemples de sept bassins fluviaux dans les Corbières et le Minervois - Géomorphologie : relief, processus, environnement n\%4 2002 ;

- Coles S. - An Introduction to Statistical Modeling of Extreme Values - Springer Series in Statistics - edition Springer-Verlag - 2001;

- Comité Français des Grands Barrages (CFGB) - Les crues de projet des barrages : méthode du Gradex - $18^{\text {ème }}$ congrès de la CIGB - Bulletin n2 du Comité França is des Grands Barrages - 1994 ;

- Commission Internationale des Grands Barrages (CIGB) - Choix de la crue de projet - Méthodes actuelles - Bulletin 82 - 1992 ;

- Dalrymple T. - Flood frequency analysis - U.S. Geol. Surv. Water Supply Pap. - 1543A -1960 ;

- Delgado J .L. - Estimation des crues de référence par approche géomorphologique - La Houille Blanche N5 - 2006 ; 
- Deroo L., de Bonviller A. - Prise en compte de l'incertitude pour le calcul des crues extrêmes $-22^{\text {ème }}$ congrès de la CIGB -2006 ;

- Diame S.-H., Srae - Synthèse nationale sur les crues des petits bassins versants Fascicule 2: la méthode Socose - Information Technique du CTGREF n\&-2 et fascicule 3 : la méthode Crupedix - 1980-1982 ;

- Duband D., Michel C., Garros H., Astier J. - Évaluation des crues extrêmes et de la crue de projet par la méthode du Gradex $-16^{\text {ème }}$ congrès de la CIGB -1988 ;

- Esposito C. - Crues de septembre 2002 sur le bassin versant du Gardon : étude comparative entre les limites de la cartographie hydrogéomorphologique et le relevé de la zone inondée - Mémoire de DEA de géomorphologie - Université de Provence AixMarseille I-2003 ;

- Galéa G., Prudhomme C. - Modèles Débit-durée-Fréquence et conceptualisation d'un hydrogramme de crue synthétique: validation sur le BVRE de Draix - Hydrologie Continentale n9-1994;

- Garavaglia F. - Méthode SCHADEX de prédétermination des crues extrêmes. Méthodologie, applications, études de sensibilité - Thèse Université de Grenoble TUE 2011 ;

- Garçon R., Bossard E., Lang M., Le Clerc S. - Revisiter la notion de scénario hydrologique de référence pour la caractérisation du risque d'inondation - Rapport commun EDF/DTG-Cemagref pour le compte du ministère de l'Écologie et du Développement Durable - Programme risque inondation RIO 1-2002 ;

- Garros Berthet H. - Preliminary PMP and PMF Computation using Bounded Distribution - Symposium de la CIGB - 1995 ;

- Garros Berthet H. - Station-year and Lombardi's approaches : Tools for estimation of design floods - Barcelona Symposium on Dam Safety - 1998 ;

- Garry G., Masson M., Ballais J.L. - Cartographie des zones inondables : approche hydrogéomorphologique - Ministère de l'Équipement et Ministère de l'Environnement Les éditions Ville et Territoires - 1996 ;

- Guillot P., Duband D. - La méthode du Gradex pour le calcul de la probabilité des crues à partir des pluies - Colloque International sur les crues et leur évaluation - Leningrad IASH - 1967 ;

- Hershfield D.M. - Estimating the Probable Maximum Precipitation - ASCE Journal of the Hydraulics Division - HYS - 1961;

- Hosking J.R.M., Wallis J.R. - Regional Frequency Analysis - Cambridge University Press - 1997a ;

- Hosking, J.R.M., Wallis J.R. - Regional Frequency Analysis : an approach based on LMoments - Cambridge University Press - 1997b ;

- House K.P., Webb R.H., Baker V.R., Levish D.R. - Ancient Floods, Modern Hazards : Principles and Applications of Paleoflood Hydrology - Water science and Application vol. 5 AGU - 2002 ; 
- Javelle P. - Caractérisation du régime des crues : le modèle débit-durée-fréquence convergent. Approche locale et régionale - Thèse de doctorat - Institut national polytechnique de Grenoble - 2001 ;

- Lambert R., Gholami M., Prunet C. - Méthodologie pour une cartographie informative des zones inondables en région Midi-Pyrénées - 1995 ;

- Lang M., Lavabre J., coord., Sauquet E., Renard B., contrib. - Estimation de la crue centennale pour les plans de prévention des risques d'inondations - Éditions Quae, Collection Update Sciences \& Technologies - ISBN 978-2-7592-0067-2 - 2007 ;

- Lavabre J., Arnaud P., Royet P., Fine J.A., Delichère S., Fang Z.X, Foussard F. Crues de projet ou cotes de projet? Exemple des barrages écrêteurs de crue du département du Gard - Colloque CFBR-SHF - 2009 ;

- Le Clerc S. - Revisiter la notion de scénario hydrologique de référence pour la caractérisation des conséquences des crues - Thèse de doctorat - Université J. Fourier Grenoble, Cemagref Lyon - 2004 ;

- Lombardi G. - Analyse fréquentielle des crues - Distributions bornées - $16^{\text {ème }}$ congrès de la CIGB, Q.63 - R.17- 1988 ;

- Margoum M., Oberlin G., Lang M., Weingartner R. - Estimation des crues rares et extrêmes : principes du modèle Agregee - Hydrologie Continentale vol.9 - 1994 ;

- Miquel J. - Guide pratique d'estimation des probabilités de crue - Eyrolles - 1984 ;

- Mulvaney T.J. - On the use of self-registering rain and flood gauges in making observation of the relation of rainfall and flood discharges in a given catchment Proceedings of the Civil Engineers n\% 4 1851;

- Naulet R., Lang M., Ouarda T., Coeur D., Bobée B., Recking A., Moussay D. - Flood frequency analysis on the Ardèche river using French documentary sources from the last two centuries - Journal of Hydrology 313(1-2) - 2005 ;

- $\quad$ Neppel L., Renard B., Lang M., Ayral P.A., Coeur D., Gaume E., Jacob N., Payrastre O., Pobanz K., Vinet F. - Flood frequency analysis using historical data : accounting for random and systematic errors - Hydrological Science Journal $55: 2-2010$;

- Organisation Mondiale de la Météorologie - Guide des pratiques hydrologiques. Acquisition et traitement des données, analyses, prévisions et autres applications OMM-N168 - 1994 ;

- Paquet E., Gailhard J., Garçon R. - Évolution de la méthode du Gradex : approche par type de temps et modélisation hydrologique - La Houille Blanche n5-2006 ;

- Parent E., Bernier J. - Bayesian POT modeling for historical data - Journal of Hydrology $274-2003$;

- Prunet C. - Géographie des zones inondables de la Dordogne Moyenne - Thèse de doctorat de Géographie-Aménagement - Université Toulouse le Mirail - 1998 ;

- Ribatet M., Sauquet E., Grésillon J.M., Ouarda T.B.M.J. - A regional Bayesian POT model for flood frequency analysis - Stochastic Environmental Research and Risk Assessment 21(4) - 2006 ; 
- $\quad$ Roche M. - Hydrologie de surface - Orstom - Ed. Gauthier-Villars - 1963 ;

- Sheffer N. A., Enzel Y., Benito G., Grodek T., Poart N., Lang M., Naulet R., Cœur D. Paleofloods and historical floods of the Ardèche river, France - Water Resources Research 39 (12) - 2003a ;

- Sheffer N. A., Enzel Y., Waldmann N., Grodek T. - Claim on largest flood on record proves false - EOS Transactions, American Geophysical session, (84), 12 - 2003b ;

- Sherman - Streamflow from rainfall by unit-graph method - Eng. News Record, 108 1932 ;

- Soil Conservation Service - National Engineering Handbook Section 4, Hydrology - US Department of Agriculture - 1972 ;

- Soil Conservation Service - Curve number (SCS-CN) methodology - Kluwer academic publishers - 2003 ;

- St-Hilaire A., Ouarda T.B.M.J., Lachance M., Bobee B., Barbet M., Bruneau P. - La régionalisation des précipitations: une revue bibliographique des développements récents - Rev. Sci. Eau 16 (1) - 2003 ;

- Ven Te Chow - Handbook of applied hydrology -Section 20 - Hydrology of Agricultural Lands - MacGraw-Hill - 1964 ;

- Vidal J.J. - Cartographie informative des zones inondables en Midi-Pyrénées. Une démarche à l'échelle de la région - Colloque national sur les risques naturels, Cemagref Grenoble - 2000 ;

- World Meteorological Organization - Manual for Estimation of Probable Maximum Precipitation - Operational Hydrology Report n1, W MO 332, second edition - 1986.

\section{Hydraulique:}

- Carlier M. - Hydraulique générale et appliquée - Eyrolles - 1986 ;

- Chanson H. - The hydraulics of stepped chutes and spillways - Taylor \& Francis 2002 ;

- Degoutte G. - Diagnostic, aménagement et gestion des rivières : hydraulique et morphologie fluviales appliquées - Éditions Tec \& Doc Lavoisier, deuxième édition revue et augmentée - 2012 ;

- Graf W., Altinakar M. - Hydraulique fluviale, tome 1 : écoulement permanent uniforme et non uniforme - Presses polytechniques et universitaires romandes - 1993 ;

- Graf W., Altinakar M. - Hydraulique fluviale, tome 2 : écoulement non permanent et phénomène de transport - Presses polytechniques et universitaires romandes - 1993 ;

- Idel'cik I.E. - Mémento des pertes de charge - coefficients de pertes de charge singulières et de pertes de charge par frottement - Eyrolles - 1999 ;

- Lencastre A. - Hydraulique générale - Eyrolles - 1996 ; 
- Post G., Chervier L., Frey J.-P., Rueff, Meilland, Maurin, Ho-Ta-Khanh - Quelques problèmes particuliers posés par les déversoirs à grande capacité : tapis de protection, dissipation d'énergie par déflecteurs et aération et cavitation produite par les écoulements à grande vitesse $-13^{\text {ème }}$ congrès de la CIGB, Q.50, R.38 - 1979 ;

- Sinniger R., Hager W. - Constructions hydrauliques - Écoulements stationnaires Presses polytechniques romandes -1989 ;

- Ulrich T. - Stability of Rock Protection on Slopes - Stabilité des protections en enrochements sur les pentes - Journal of Hydraulic Engineering, Vol. 113 n7 - 1987;

- US Army Corps of Engineers - Hydraulic design of spillways - Engineer Manual 11102-1603;

- Ven Te Chow - Open-channels hydraulics - MacGraw-Hill - 1959.

\section{Boudins, hausses:}

- Degoutte G., Royet P., Alonso E. - Seuil souples - Utilisations en rivière et sur les barrages - Informations techniques du Cemagref n5, note 2-1992 ;

- Kennedy D. - Inflatable storage - International Water Power \& Dam Construction 2004 ;

- Royet P., Degoutte G. - Hausses fusibles sur les déversoirs de barrages - Informations techniques du Cemagref n85, note 1-1992.

\section{Accidentologie :}

- HYDRO - 2009 - Lyon ;

- Diacon A., Stematiu D., Mircea N. - An analyse of the Belci dam failure - Water Power \& Dam Construction - 1992.

Vent :

- Centre Scientifique et Technique du Bâtiment (CSTB) - Règles NV 65 : règles définissant les effets de la neige et du vent sur les constructions et annexes - DTU P06002 - AFNOR - 2009 ;

- Commission Internationale des Grands Barrages (CIGB) - Barrages en remblai, Protection du talus amont - bulletin n91 - 1993 ;

- Institution of Civil Engineers (ICE) - Floods and reservoir Safety - third edition Thomas Telford Publications - 1989 ;

- Naaim M., Degoutte G., Delorme F. - Les vagues dans les retenues d'altitudes: analyse et méthodes pour la prévention - Sciences Eaux \& Territoires n02 - 2010 ; http://www.set-revue.fr/sites/default/files/archives/set 02 article $10 \quad 0 . p d f$ 
- Saville T., McClendon E., Cochran A. - Freeboard Allowances for Waves in Inland Reservoirs - Journal of the Waterways and Harbours Division, ASCE - 1962 ;

- Smith, J. - Wind Wave Generation on Restricted Fetches - Coastal Engineering Research Center, Waterways Experiment Station, Vicksburg, MS - 1991;

- South African Committee On Large Dams (SACOLD) - Interim guidelines on Freeboard for dams, Safety evaluation of dams - 1990 ;

- US Army Corps of Engineers - Shore protection manual - Waterways Experiment Station, Vicksburg, MS - 1984. 